\title{
EMPIRICAL EVIDENCE AGAINST PHENOMENAL THESES
}

\author{
BY
}

\section{EYYUPHAN OZDEMIR}

\author{
A thesis \\ submitted to the Victoria University of Wellington \\ in fulfilment of the requirements for the degree of \\ Doctor of Philosophy
}

Victoria University of Wellington

2022

This research has been approved by the Victoria University of Wellington Human Ethics Committee [0000026962] 
To my wife and best friend Songül, 


\section{ABSTRACT}

Classical anti-physicalist reasoning about phenomenal consciousness involves two related theses: (1) phenomenal consciousness exists, and (2) the existence of phenomenal consciousness generates an epistemic gap. From the epistemic gap between the physical and the phenomenal, the anti-physicalist then concludes that there is a corresponding ontological gap. Defences of the existence of these two phenomenal theses rely heavily on appeals to intuitions. Further, the phenomenal intuitions at issue are typically taken to be widespread in the population, and not only by anti-physicalists but also by a majority of physicalists. I will show, first, that the most common way of defending the phenomenal theses is by appeal to phenomenal intuitions. Second, I will present empirical evidence indicating that these phenomenal intuitions are not in fact widespread. Finally, I argue that the fact that phenomenal intuitions are not widespread undercuts the most common defence of the phenomenal theses offered by anti-physicalists. In total, this dissertation lays out and defends a general argument against the theses that phenomenal consciousness exists and generates an epistemic gap: the main way of defending phenomenal theses fails because the arguments given in favour of each ultimately require phenomenal intuitions to be natural and thus widespread, but the evidence indicates that they are not. This evidence, in turn, has direct implications for a further recent philosophical debate surrounding the supposed meta-problem of consciousness, which is also motivated by the assumption that phenomenal intuitions are widespread or, at least, have a widely shared basis. I conclude by arguing that the evidence concerning phenomenal intuitions suggests that this assumption is false, and that this renders the problem both less interesting and less important than has been assumed. I conclude that even if explaining the phenomenal intuitions of the minority remains a problem, the evidence presented in this dissertation raises a much more significant problem for those who defend qualia realism. The reverse meta-problem raises the question: Why should we believe that phenomenal consciousness exists given the paucity of intuitive support for it? 


\section{ACKNOWLEDGEMENTS}

My adorable wife, Songül, deserves the most special thanks for all her support and patience during my research. Without her encouragement, I would not have found the energy to take on this enormous endeavour, and without her emotional support, I would not have finished this research. Whenever I felt stuck, she made me look at the problems from a different perspective with a coolheaded brain. My other families, the ones in Turkey, Ozdemirs and Godfreys, I have always felt your irreplaceable support, which has made everything easier here, including my doctoral research. Thanks for being so benevolent and supportive of any adventure of mine and Songül's.

I cannot express my gratitude enough to my primary supervisor, Justin Sytsma. Without his guidance, it would have been extremely difficult to see the finish line. He has been helpful, not only for reviewing the philosophical content, but also for the statistical analysis involved. It is a cliché to say, I know, but it is completely true: I have been incredibly lucky to have him on this great but difficult and long journey. And thanks to Eugen Fischer, my secondary supervisor, for his support.

I would also like to thank all the academics of the Philosophy Department of VUW, especially Simon Keller, Cei Maslen, and Sondra Bacharach, for helping me when I needed support during my study. Thanks, also, to Xavier Marquez and Jonette Crysell who have been very helpful to me in overcoming various kinds of difficulties. I also thank Ayca Arkilic, Manjeet Pardesi, Caroline Bennett, and Catherine Abou-Nemeh for providing further academic guidance, for sharing individual experiences of their doctoral research with me, and for their invaluable friendship, which has made my research easier. Jonathan Pengelly, my office mate and lovely friend, also deserves special thanks, for listening to key ideas of the thesis, commenting on them, and providing the motivation that I needed during the painful stages of my research.

Lastly, Professor Stephen Voss, my advisor in my Master's research, I will never forget your invaluable, multifaceted, and sophisticated support, encouragement and all the things you taught me, especially self-critical thinking, and charitable reading of and fairness to opposite views. You and Justin will always be my guiding stars in philosophy. 


\section{TABLE OF CONTENTS}

$\underline{1}$ INTRODUCTION

$\begin{array}{lll}1.1 & \text { The General Argument } & 3\end{array}$

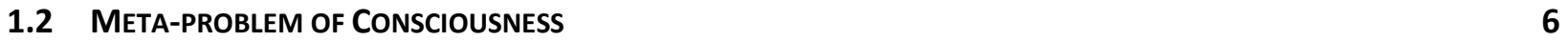

2 THE CENTRAL ROLE OF INTUITIONS IN DEFENCE OF PHENOMENAL REALISM

$\begin{array}{llr}2.1 & \text { Phenomenal Realism } & 9\end{array}$

2.2 The Claims that Phenomenal Consciousness is Pretheoretically Obvious

2.3 The Connection between the Pretheoretical Obviousness of Phenomenal Consciousness and INTUITIONS

2.3.1 INDIVIDUALIST VERSUS COLLECTIVIST WAY OF APPEALING TO EXISTENCE INTUITIONS

2.3.2 Why Appealing to Existence Intuitions is the Primary Way of Defending Phenomenal Realism

2.4 Concluding Remarks

3.1 The EPISTEMic GaP Thesis 
6 A GAP BETWEEN PERCEPTUAL STATES AND OTHER “PHENOMENAL" STATES IN THE FOLK CONCEPTION OF MIND 
10.1 A Brief Summary of the General Argument

10.2 IMPLICATIONS FOR THE MeTA-PROBLEM OF CONSCIOUSNESS

10.2.1 IMPLICATIONS OF THE SCARCITY OF EXISTENCE INTUITIONS ON META-PROBLEM 


\section{LIST OF FIGURES}

Figure 1: Comparison between the results of Fiala et al. (2014) and four studies of Sytsma (2014).

Figure 2: Results in Ambiguity Study 1 with 95\% Cls; histograms above respective graphs.

Figure 3: Mean responses of Timmy's and Jimmy's seeing red in Ambiguity Study 2 with 95\% confidence intervals together with the corresponding histograms.

Figure 4: Mean responses for Jimmy sees/detects auditorily and visually in Ambiguity Study 3 together with the corresponding histograms.

Figure 5: Mean responses for six vignettes in Ambiguity Study 4 with corresponding histograms above. 100

Figure 6: 13 agents located in the two-dimensional mental coordinate system in Gray et al. (2007). 107

Figure 7: The mean responses for supposedly phenomenal states in Dimension Study 2. 126

Figure 8: The coordinates of mental capabilities in two dimensions indicated in Dimension Study 2. 


\section{LIST OF TABLES}

Table 1: Factor loadings of mental capabilities in Dimension Study 1...........................................................119

Table 2: Factor loadings of 25 statements in Dimension Study 2....................................................................128

Table 3: Proportions of the participants who endorse that philosophical zombies are possible in Study 4 of Fischer and

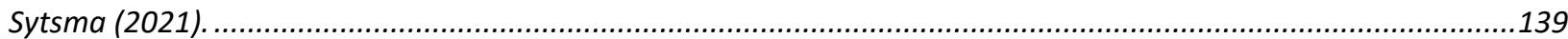




\section{INTRODUCTION}

This thesis concerns the position of phenomenal realism and the epistemic conclusions often thought to follow from it. By "phenomenal realism" I mean the thesis that phenomenal consciousness exists. Phenomenal consciousness is then typically defined in terms of the possession of mental states falling under a distinctive class, with the mental states forming this class being taken to have something distinctive in common - the possession of phenomenal properties or qualia. Let us refer to such mental states, neutrally, as subjective experiences (or "experiences" for short), leaving open whether they have phenomenal properties. Perceptual cases (seeing colors or smelling a rose and so on) and bodily sensations (feeling pain, orgasm, itchiness, and so on) are the least controversial and most prototypical examples of these subjective experiences. ${ }^{1}$ Phenomenal realists hold that these mental states form a class because each has a distinctive subjective feel or phenomenal character that we are acquainted with. Put another way, it is thought that there is something it is like to be in each of these states (Chalmers, 1995, p. 201; Nagel, 1974, p. 436). The underlying idea here is that such sensory properties (redness, painfulness, and so on) are properties of mental states (or of "experiences"), not properties of the objects perceived or represented. To say that these properties are phenomenal properties, and that the corresponding experiences are phenomenal experiences, then, is to take them to be properties of experiences (Block, 2004; Byrne, 2004; Carruthers, 2001; Chalmers, 2002; Kind, 2020). And such phenomenal properties are then believed to characterize the phenomenology of subjective experiences.

A great deal of effort in the philosophy of mind, cognitive science and neuroscience has gone into discussions of and arguments about phenomenal consciousness. I will focus on two critical theses in these debates, which I term "phenomenal theses": The first is that phenomenal consciousness exists (phenomenal realism), the second that there is an epistemic gap between the phenomenal and the physical (the epistemic gap thesis). The theses of phenomenal realism and the epistemic gap are the foundations of classical anti-physicalist reasoning, which culminates in the antiphysicalist conclusion that there is a further ontological gap between phenomenal consciousness

\footnotetext{
${ }^{1}$ Felt thoughts, emotions, and moods are also added to this list by some philosophers (Block, 2002; Chalmers, 2003).
} 
and the physical. While this too might be classified as a phenomenal thesis (the ontological gap thesis), I will focus on just the prior foundational theses in this dissertation, taking "phenomenal theses" to be restricted to them. Proponents of these phenomenal theses have called on intuitions about phenomenal consciousness ("phenomenal intuitions" hereafter) to defend these theses. Opponents have, in turn, usually tried to show that phenomenal intuitions are misleading, or that even if they are true, they do not support the phenomenal thesis that they are typically taken to support.

I will adopt another approach, however, arguing that there is an empirical way to assess the plausibility of phenomenal theses. In this dissertation, I contend that phenomenal theses require phenomenal intuitions to be prevalent for the theses to be plausible. That is, the intuitions of the proponents of phenomenal realism and the epistemic gap are compelling evidence for the theses only if these intuitions are shared by the clear majority of non-philosophers. In line with this, appeals to phenomenal intuitions often include (implicitly or explicitly) empirical claims about the prevalence of these intuitions, as will be seen in the next two chapters. For instance, Chalmers (1995) maintains that phenomenal consciousness is "the most central and manifest aspect of our mental lives". To give another example, this time from the physicalist camp, Papineau (2006) contends that "intuitively we feel that there are zombie worlds" in which molecule-for-molecule copies of humans lack an inner, phenomenal life. In this way, phenomenal theses are claimed to be supported by premises that are taken to be phenomenologically obvious and, hence, pretheoretically obvious. Put another way, it is often held that phenomenal intuitions are rooted in common sense or folk psychology. Whether this is true or not is an empirical question, however. And the corresponding empirical claims can be assessed using the tools of experimental philosophy (Sytsma \& Livengood, 2015). If the empirical claims turn out to be incorrect, I will argue, this will dramatically undermine the plausibility of the phenomenal theses. This possibility paves the way for building a general, empirically informed argument that attacks phenomenal theses from an evidential point of view. In this dissertation, I build and defend such an argument.

In the remainder of this chapter, I will introduce the General Argument and explains its premises (§1.1), then introduce the meta-problem of consciousness (Chalmers, 2018) and explain how the General Argument (and specifically Premise 2 of that argument) bears on this further issue $(\S 1.2)$. 


\subsection{The General Argument}

To repeat, let us call the theses of phenomenal realism and the epistemic gap "phenomenal theses". Phenomenal theses can be argued for directly or indirectly via well-known arguments, which I will call "phenomenal arguments". For example, the epistemic gap thesis is often supported via epistemic gap arguments such as the Knowledge Argument (Jackson, 1982). Regardless of approach, I will show that the support for these phenomenal theses commonly hinges on appeals to phenomenal intuitions. That is, the primary way of defending phenomenal theses is by appealing to phenomenal intuitions. With this setup in place, the General Argument can be laid out as follows:

(Premise 1): Phenomenal theses (phenomenal realism and the epistemic gap thesis) are primarily defended by appealing to phenomenal intuitions.

(Premise 2): Phenomenal intuitions are not prevalent.

(Premise 3): Appeals to phenomenal intuitions that are not prevalent do not provide compelling evidence for phenomenal theses.

Conclusion: The primary way of defending phenomenal theses fails.

Before defending this argument, it is important to explain what is meant by "intuition". Inspired by the core idea of the family of accounts of intuition that Pust (2019, 1.3 section) labels "Intuition as Sui Generis States", I will be operating with the following conception of intuitions:

A subject $\mathrm{S}$ has the intuition that $\mathrm{P}$ if $\mathrm{P}$ seems true to $\mathrm{S}$ via implicit reasons. That is, $\mathrm{P}$ should not seem to $\mathrm{S}$ true as a result of inference or any explicit reason to believe $\mathrm{P}$.

This view about intuitions has two merits: First, one can have the intuition that $\mathrm{P}$ and yet disbelieve $\mathrm{P}$, so believing $\mathrm{P}$ is not necessary for $\mathrm{S}$ to have an intuition that $\mathrm{P}$. For example, I still feel the intuition that it does not matter whether you switch doors in the Monty Hall puzzle, even though I firmly believe that this is false. While this view of intuitions avoids the claim that believing $\mathrm{P}$ is a necessary condition for having the intuition that $\mathrm{P}$, it allows that if one has the intuition that $\mathrm{P}$, and if there is no reason to disbelieve $P$, that the person will most likely believe $\mathrm{P}$. Second, the view also avoids the main problem for dispositionalist accounts of intuition. On the dispositional account of intuitions, a person has the intuition that $\mathrm{P}$ if and only if that person is disposed to 
believe $\mathrm{P}$ merely on the basis of understanding $\mathrm{P}$. The problem with this view is that having an intuition seems to require one to be in a conscious, occurrent psychological state and one's having the disposition to believe P does not guarantee that one is in such a state (Pust, 2019). The view I favour is congruent with the possibility that one might never host an intuition that $\mathrm{P}$ even if one is disposed to believe that $\mathrm{P}$ because it claims that $\mathrm{S}$ has the intuition that $\mathrm{P}$ only when $\mathrm{P}$ seems true to $\mathrm{S}$. However, the view still involves the plausible idea that when a subject has an intuition that $\mathrm{P}$, she must have been disposed to believe that $\mathrm{P}$ because of some implicit reasons.

On this view of intuitions, a proposition seeming true to $\mathrm{S}$ means that $\mathrm{P}$ is presented to $\mathrm{S}$ as true, or S is pushed to believe $\mathrm{P}$ without S's awareness of underlying reasons for $\mathrm{P}$. Note, however, that on this view, not every seeming is taken to be an intuition. The proposition P must seem true to $\mathrm{S}$ without any underlying, supporting reason that S. If I see that an airplane is falling down and if I believe that it will crash, this does not mean that I have the intuition that it will crash. The reason is that I have a perceptual reason of which I am aware that supports the belief that the plane will crash. However, when I see the airplane flying in a normal way and yet it nonetheless seems to me that it will crash, then I have an intuition because I am aware of no reason to have this belief.

Note further that the view of intuitions I have offered does not constitute a full definition of intuitions because it does not indicate any necessary condition for $S$ to have an intuition while stating only one sufficient condition for having an intuition. So, there might be different situations that can produce intuitions. However, the intuitions that play a significant role in causing one to have beliefs that are suggestive of phenomenal realism and the epistemic gap, seem to be wellcaptured by this view of intuition since what is usually claimed in favour of phenomenal theses is that it seems, for example, that some mental states have qualia while others lack qualia, or that there is an explanatory gap between the physical and the phenomenal, or that some people may see objects in a systematically inverted way even if all the relevant physical factors are the same, with no underlying reason for this seeming that the believer is aware of.

With this understanding of intuitions in hand, here is how I will defend the General Argument in the remainder of this thesis. Premise 1 claims that the most common way to defend phenomenal theses is by appealing to intuitions that individually or collectively reflect that the phenomenal thesis in question is true. The claim is that by far the most common defence offered for phenomenal 
theses ultimately rests on asserting some beliefs as seeming obviously true to the proponent of the thesis. As will be detailed in Chapter 2, there are two ways of appealing to intuitions to defend phenomenal theses: In the individualist way, the philosopher defends the thesis in question by relying on her own intuitions while in the collectivist way, the philosopher defends the thesis by appealing to the prevalence of the phenomenal intuitions.

To establish Premise 1, Chapter 2 and 3 will detail the central role of intuitions in defending phenomenal realism and the epistemic gap thesis, respectively, based on textual evidence. In the case of phenomenal realism, Premise 1 amounts to saying that the most common way to defend phenomenal realism is by claiming that phenomenal consciousness is pretheoretically obvious and thus intuitively true, or, reversely, that the denial of phenomenal consciousness is counterintuitive. That is, proponents of phenomenal realism usually express, albeit in slightly different ways, the idea that the existence of phenomenal consciousness is pretheoretically obvious - that it is, for instance, phenomenologically, psychologically, or introspectively obvious or that is otherwise a clear part of common sense or folk psychology — and on the basis of this that the existence of phenomenal consciousness can be taken for granted or that phenomenal realism should have default status in the debates. As I will explain in detail in Chapter 2, if a person thinks that the existence of phenomenal consciousness is pretheoretically obvious, then that person has the intuition that phenomenal consciousness exists. As for the epistemic gap thesis, the connection between intuitions and the thesis is still clearer: the thesis is typically presented in epistemic gap arguments (such as the Knowledge Argument and conceivability arguments) that have a crucial premise pumping a certain sort of intuition (e.g. the philosophical zombie intuition that moleculefor-molecule copies might not be able to see colors or feel pain, or the knowledge intuition that even if we had the complete set of physical facts, we would not be able to know what it is like to see red if we had never had a visual experience of that type before). 
Premise 2 is an empirical premise that holds that phenomenal intuitions are not prevalent. By this I mean that these intuitions are not shared by the majority of laypeople. That is, the premise makes the empirical claim that only a minority of people have phenomenal intuitions. ${ }^{2}$

Since empirical premises call for empirical support, a critical step in this dissertation will be to survey and add to the empirical evidence concerning phenomenal intuitions. This will be done in Chapters $4-8$ by discussing relevant empirical studies from the literature, as well as by presenting the results of eight new studies with seven being cross-cultural (total $\mathrm{N}=2,414$ ). The findings make the case that laypeople, by and large, do not share the phenomenal intuitions, supporting Premise 2 and in turn Premise 3. However, both premises rely on some assumptions. These include that no special introspective skills or conceptual training is needed to have phenomenal intuitions and that careful reflection on questions related to phenomenal experiences would not make one more likely to have or report phenomenal intuitions. So, a proper defence of Premise 2 and Premise 3 should include replies to some existing and possible objections against such assumptions. This is done in the first part of Chapter 9.

In the second part of Chapter 9, I then defend Premise 3, arguing that regardless of whether a philosopher appeals to phenomenal intuitions in the collectivist or individualist way, the phenomenal intuitions of that philosopher do not provide any substantial support for phenomenal theses if their intuitions are not generally prevalent.

\subsection{Meta-problem of Consciousness}

In a recent article, Chalmers (2018) presents a new problem of consciousness-what he terms the "meta-problem". The meta-problem of consciousness is the (supposed) problem of explaining why people think that phenomenal consciousness poses a hard problem for the physical sciences, or

\footnotetext{
${ }^{2}$ If phenomenal realism is pretheoretically obvious and the epistemic gap claims are intuitively true, we would expect that a clear majority of people have phenomenal intuitions. However, even a bare majority of people who have phenomenal intuitions provide evidence (albeit minimal and defeasible) that these intuitions are common not only among philosophers but also among ordinary people, and that they have a common, phenomenal consciousnessrelated basis. So, Premise 3 in this case would not be easy to maintain. That is why Premise 2 holds that people who have phenomenal intuitions are not the majority rather than that they are not the clear majority.
} 
why people have phenomenal intuitions reflecting that there is a hard problem. Chalmers calls these "problem intuitions" and takes them to include four central types: explanatory, metaphysical, knowledge, and modal intuitions. Chalmers believes that all these central problem intuitions are widely shared and well beyond philosophers (Chalmers, 2018, pp. 13, 15). And this reflects that he holds that "we" think that there is a hard problem of consciousness. That is, put in the terminology introduced in the previous section, Chalmers assumes that Premise 2 of the General Argument is false. Thus, the empirical evidence presented in Chapters 4-8 has direct implications for the existence and importance of the (supposed) meta-problem. Chalmers also proposes a working assumption to the effect that when problem intuitions are present, this is due to some nearuniversal factors (phenomenal states and introspective mechanisms) and when they are not present it is due to some variable factors ("e.g. cultural, linguistic, and theoretical background, and other factors that vary with historical period and individual psychology" (Chalmers, 2018, p. 15). He maintains that even if his working assumption is false, the meta-problem remains an important research project and holds that the meta-problem poses a challenge for any theory of consciousness - that any such theory should explain our problem intuitions.

In Chapter 10, after summarizing why the evidence presented in this thesis supports the conclusion of the General Argument, I will discuss the implications of the evidence for the meta-problem. I will first note that the experimental studies discussed across this thesis already suggest that problem intuitions are not prevalent. Based on this, I argue that if the meta-problem is understood as the problem of explaining why "we" have problem intuitions, there is no such problem simply because we by and large lack these intuitions!

Chalmers, however, also offers a more modest definition of the problem. More modestly, it is the problem of explaining what problem intuitions do occur, however widespread or scarce they might be. Although I agree that this modest version of the meta-problem survives the scarcity of problem intuitions as demonstrated in Chapters 4-8, I will claim that resolving the modest version of the meta-problem is far less important than Chalmers contends. That phenomenal intuitions are not prevalent suggests that Chalmers's working assumption is false, which makes the meta-problem challenge inapposite. If there is a challenge for phenomenal realists related to the meta-problem, it is not the meta-problem challenge but the reverse meta-problem challenge: any realist theory of phenomenal consciousness should explain why we should believe that phenomenal consciousness 
exists considering that there is no meta-problem in its presumptuous sense. In other words, realists should explain why we should believe that phenomenal consciousness exists in the first place if the conclusion of the General Argument is true. 


\section{THE CENTRAL ROLE OF INTUITIONS IN DEFENCE OF PHENOMENAL REALISM}

Remember that the main argument of the thesis, namely the General Argument, was presented in the Introduction as follows:

(Premise 1): Phenomenal theses (the phenomenal realism and epistemic gap theses) are primarily defended by appealing to phenomenal intuitions.

(Premise 2): Phenomenal intuitions are not prevalent.

(Premise 3): Appeals to phenomenal intuitions that are not prevalent do not provide compelling evidence for phenomenal theses.

Conclusion: The primary way of defending phenomenal theses fails.

This chapter aims to defend the first premise of the General Argument with regard to the phenomenal realism thesis. $\$ 2.1$ will clarify this thesis. $\$ 2.2$ will show that phenomenal realism is commonly supported by claims that phenomenal consciousness is pretheoretically obvious. $§ 2.3$ will argue that this talk of phenomenal consciousness being pretheoretically obvious is best understood as an appeal to intuitions and show that intuitions are the primary support offered for phenomenal realism, although there are two different ways in which these philosophers could be intending these appeals to intuitions to go.

\subsection{Phenomenal Realism}

As stated in the Introduction, phenomenal realism is inseparably connected to qualia: the properties of experiences that characterize what it is like to undergo a phenomenal experience. As such, phenomenal realism is the view that qualia exist, where qualia are understood as phenomenal properties of experiences. However, "qualia" has been defined in different ways in the literature, and thus there are different versions of phenomenal realism depending on the definition one adopts.

Following Bailey (2007, p. 88), there is a topic-neutral sense of "qualia" on which the term refers to "colors as they are presented to us in visual sensation, tastes as we experience them, sounds as we hear them, and so on". As Bailey points out, in this sense, "it is not part of the meaning of the term that qualia are particularly 'mental' or 'internal' or 'subjective' properties, let alone ineffable, 
intrinsic, private and directly apprehensible” (p. 88). Dennett (1988), for example, seems to believe in qualia only in this sense when he states that " Qualia' is an unfamiliar term for something that could not be more familiar to each of us: the way things seem to us" (p. 1). This sense of qualia allows that they might not be properties of mental states/experiences. Thus, philosophers, such as Dretske (2003), Tye (2000) and Campbell (2002), who believe that qualia are properties of external objects rather than of experiences/mental states, can also be taken to be using "qualia" in this topicneutral sense. According to these philosophers, the quale of redness, for example, belongs to the red object we see rather than to our visual experience. For instance, Dretske (2003, p.67), defines qualia "a subset of objective physical properties - what used to be called observational properties".

The topic-neutral sense of qualia is not common in literature, however. Typically, when philosophers or scientists talk about qualia, they use the term at least in the sense of phenomenal properties of mental states/experiences (among many others see, e.g., Block, 2004; Byrne, 2004; Carruthers, 2001; Chalmers, 2002; Crick \& Clark, 1994; Dennett, 1991; Kind, 2020; Koch, 2004). To illustrate, Byrne (2004, p. 204) gives a concise explanation of this sense of qualia:

For what follows, an example of a Blockean "experiential property" will be useful. Consider visual experiences (in good light) of cucumbers, limes, green peppers, grass, and so on. These experiences saliently resemble each other in respect of "what it's like" to undergo them; they accordingly share an experiential property, call it ' $G$ '. So-called spectrum inversion is-at least-a case where experiential properties are systematically permuted. When an inverted subject looks at a tomato, his experience has G. Sometimes a property like $\mathrm{G}$ is called a 'phenomenal character', a 'qualitative character', or a 'quale'. The last two expressions are used in this way in Levine 2001; in the additional terminology of that book, G is "greenishness".

As can be noted from this quote, unlike the topic-neutral sense, in Byrne's sense qualia are properties of experiences that determine the phenomenal character of those experiences. Phenomenal redness, for example, is a quale of our visual sensation. Since this sense still does not imply anything further about the metaphysical features of qualia - such as whether qualia are intrinsic, ineffable, atomic, subjective, non-physical - we can still think of this as a "minimal" 
sense of qualia. This minimal sense, which Frankish (2012) calls "diet qualia", is endorsed by the majority of physicalist realists. There is also a more demanding sense of qualia which Frankish calls "classic qualia" and Dennett (1988) calls "traditional qualia". In this more demanding sense, qualia are intrinsic, ineffable, private, simple (atomic or monadic), subjective and introspectable properties of phenomenal experiences. Most anti-physicalists endorse this classical sense of qualia.

I will use the term "qualia" in the minimal sense. So, given this definition of qualia, phenomenal realism, in the thesis, will refer to the view that subjective qualia exist at least in the minimal sense, which includes the classical sense but excludes the topic-neutral sense of qualia.

\subsection{The Claims that Phenomenal Consciousness is Pretheoretically Obvious}

The most prominent philosophers working on phenomenal consciousness usually take the existence of phenomenal experiences for granted, claiming that their existence is pretheoretically obvious, although they often express this in slightly different ways. The basic idea in each case, however, is that it is simply obvious that some mental states have qualia while other mental states lack them, and this is taken to be obvious not based on consideration of sophisticated philosophical arguments, or based on supporting scientific evidence, or deep reflection on the nature of experience, but prior to and independent of any such considerations. The existence of qualia is taken to be obvious from the get-go. As Jaworski notes, "Those who believe in qualia (phenomenal character/properties of experiences) share a sense that the existence of qualia is too obvious to require argument" (Jaworski, 2011, p. 215).

In this section, I will detail just a few of the multitude of examples of claims that qualia are pretheoretically obvious offered by phenomenal realists. First, consider Stoljar (2006)'s claim that phenomenal consciousness is phenomenologically obvious: "Apart from its phenomenological obviousness, the two central facts about conscious experience are these: It is philosophically puzzling, and we are scientifically ignorant with respect to it" (p. V). Here Stoljar's claim is that phenomenal consciousness ("conscious experience" in his terminology) is phenomenologically obvious can be taken as the view that phenomenal consciousness is pretheoretically obvious 
because if something is phenomenologically obvious, then there is no need for a theory attempting to show that it exists. ${ }^{3}$

Next, consider the following assertion from Schwitzgebel (2016): "Phenomenal consciousness is the most folk-psychologically obvious thing or feature that the positive examples possess and that the negative examples lack" (p. 229). ${ }^{4}$ If Schwitzgebel is right, phenomenal experiences are simply there and everyone is aware of them, with the result that there is no need for us to call on theory to be convinced that there are phenomenal experiences.

Kind (2020, para. 4) makes a similar claim, asserting that "from the standpoint of introspection, the existence of qualia seems indisputable". This means that when one introspects on one's experiences, qualia manifest themselves without any doubt, in which case again, we do not need to call on theory to be convinced that qualia exist. The only thing that we need to see that qualia exist is to introspect on our experiences.

Or consider Chalmers's (1995, p. 207) claim, noted above, that "experience is the most central and manifest aspect of our mental lives, and indeed is perhaps the key explanandum in the science of the mind". Chalmers here clearly takes the existence of phenomenal consciousness for granted: it is the starting point, not something whose existence is to be inferred or whose existence could reasonably be brought into question.

\footnotetext{
${ }^{3}$ It should be noted here that it is common for phenomenal realists to use the term "experience" or "conscious experience" as a synonym for phenomenally conscious states. For example, in the same work, Stoljar says that "The idea that experiences are events and that phenomenal character is the defining property of such events raises questions of a very general nature about what exactly events are and what exactly it is to be a property of an event (or of anything else for that matter)" (p. 21). For another example, after labelling phenomenal consciousness as P-Consciousness, Block says: "P-consciousness is experience. P-conscious properties are experiential properties. P-conscious states are experiential states; that is, a state is P-conscious just in case it has experiential properties. The totality of the experiential properties of a state are 'what it is like' to have it. Moving from synonyms to examples, we have Pconscious states when we see, hear, smell, taste and have pains" (Block, 2002, p. 207). Like Block, Chalmers also equates phenomenal consciousness with the term "experience" and clearly states that he uses the terms "experience" (or "conscious experience") and "phenomenal consciousness" interchangeably. He also equates experience with whatit-is-like states (Chalmers, 1995, 2018).

${ }^{4}$ Schwitzgebel (2016) lists sensory experiences, imagery experiences, vivid emotions, and dreams as positive examples while listing growth hormone release, dispositional knowledge, standing intentions, and sensory reactivity to masked visual displays as negative examples (pp. 227-229).
} 
Finally, consider Block's (1978, p. 281) suggestion that the existence of phenomenal consciousness is something that can be recognized easily, answering the rhetorical question "What is it that philosophers have called qualitative states?" with a remark given by Louis Armstrong about jazz: "If you got to ask, you ain't never gonna get to know." Again, the clear sense is that the existence of phenomenal consciousness is pretheoretically obvious; it isn't something you need to work out or infer from careful reasoning, but something that you immediately know. And many, many more examples could be given from the literature.

Does defending phenomenal realism by claiming that phenomenal consciousness is pretheoretically obvious - that it is obvious from our phenomenology or basic introspectionmean that phenomenal realism is primarily defended by appealing to intuitions? In the rest of this chapter, I will first show that the talk of phenomenal consciousness being pretheoretically obvious is best understood as appealing to intuitions, then show that this is the primary way of defending phenomenal realism.

\subsection{The Connection between the Pretheoretical Obviousness of Phenomenal Consciousness and Intuitions}

We've seen that it is common to defend phenomenal realism by appeal to the claimed pretheoretical obviousness of phenomenal consciousness and qualia. I now argue that this common practice amounts to an appeal to intuitions as they are articulated in the Introduction. That is, I will argue that phenomenal realism is commonly defended by appeal to what I will term "existence intuitions"-intuitions supporting the claim that phenomenal consciousness exists.

First, it is worth noting that talk of "intuitions" is abundant in the literature, with both phenomenal realists and sceptics often treating the debate as centring on intuitions. For instance, as noted above, Chalmers (2018, pp. 12-13) articulates the meta-problem in terms of problem intuitions, including intuitions concerning the existence of qualia and an epistemic (explanatory, knowledge and modal) gap. And this is not atypical. For example, Chalmers (2003) states that the debate between TypeA materialism and its opponents "usually comes down to intuition" including "the intuition that consciousness (in a nonfunctionally defined sense) exists" (p. 114) and claims that to deny the existence of phenomenal consciousness (or that it poses an epistemic gap) "would be to make a 
highly counterintuitive claim that begs the important questions" (p. 112). ${ }^{5}$ This fits with his talk about intuitions about experiences that are suggestive of the existence of qualia such as “metaphysical”, “quality” and "distribution” intuitions (Chalmers, 2018, pp. 12-13).

To give other examples from the literature that point out the relationship between intuitions and phenomenal realism, Dennett $(1988$, p. 227) states that the main goal of his article is "to destroy our faith in the pretheoretical or 'intuitive' concept of qualia" and mentions two central intuitions that he labels as "intuition pumps". ${ }^{6}$ Frankish (2012) states that "If we reject classic qualia realism, then all our intuitions about qualia need explaining away, including the intuition that experiences have a phenomenal character at all" (p. 675). He also mentions our potent and realist intuitions and states that if the Illusionist research programme "proves fruitful, then the realist intuition may loosen its grip on us (or we may loosen our grip on it)" (Frankish, 2016b, p. 257). ${ }^{7}$ Leeds (1993) calls the intuitions "that lead many of us to think of sensory impressions as having qualitative content" as "qualia intuitions" (p. 303).

Second, on the account of intuitions offered above, appeals to the pretheoretical obviousness of phenomenal consciousness and qualia amount to an appeal to intuitions. Recall that my preferred account offers a sufficient condition for being an intuition:

A subject $\mathrm{S}$ has the intuition that $\mathrm{P}$ if $\mathrm{P}$ seems true to $\mathrm{S}$ as long as $\mathrm{S}$ believes $\mathrm{P}$ not via inference or an explicit reason to believe P.

\footnotetext{
${ }^{5}$ Chalmers defines Type-A materialism as the view that "there is no epistemic gap between physical and phenomenal truths, or at least any apparent epistemic gap is easily closed" (p. 112). The view may take the form of Eliminativism (the view that phenomenal consciousness does not exist), or Analytical Functionalism ("concept of "consciousness" is defined in wholly functional or behavioural terms"), or any view according to which explaining "cognitive, behavioural, and environmental functions" suffices to explain phenomenal consciousness (p. 112).

${ }^{6}$ In intuition pump \#1 ("watching you eat cauliflower"), it seems natural for us to distinguish "subjective tastes, looks, aromas, sounds" as "independently of how those individuals are stimulated or non-perceptually affected, and independently of how they are subsequently disposed to behave or believe". The intuition pump \#2 ("the wine tasting machine") reflects that, since the qualitative property in our tasting experiences seem to be isolated from the causes of the taste we experience and the related dispositions to believe and act in a particular way, a wine-tasting machine that "might well perform better than human wine tasters on all reasonable tests of accuracy and consistency", no matter how 'sensitive' and 'discriminating' it is, "it will never have, and enjoy, what we do when we taste a wine: the qualia of conscious experience! Whatever informational, dispositional, functional properties its internal states have, none of them will be special in the way qualia are". Dennett plausibly contends that if one shares these intuitions, then she believes that there are qualia in the sense of intrinsic properties of subjective experiences.

${ }^{7}$ Illusionism is the view that "phenomenal consciousness is illusory" (Frankish, 2016a, p. 11).
} 
Accepting this condition, the connection between the talk of phenomenal consciousness being pretheoretically obvious and intuitions is clear. The condition holds that one's believing a statement without inference or any explicit reason is sufficient for having an intuition that this is the case. The claim that phenomenal consciousness is pretheoretically obvious (that it is phenomenologically, psychologically, or introspectively obvious), however, implies that the (supposed) phenomenality of experiences seems obvious to the philosopher who makes the claim, and this recognition disposes her to have some beliefs that accord with the core claims of phenomenal realism, such as the claim that pain is in our mind, that there is something that it is like to feel pain and see colors, and so on. In other words, claiming that phenomenal consciousness is pretheoretically obvious is claiming that the existence of phenomenal consciousness strikes one as obvious without any inference, argument, or explicit reason. This is a situation that exactly fits with the sufficient condition of the intuition presented above. So, it makes sense to treat the claim that phenomenal consciousness is pretheoretically obvious as an intuition-based claim.

The upshot is that it is hardly presumptuous to claim that intuitions play a role in defending phenomenal realism. But this does not yet make clear how such intuitions are supposed to provide support for phenomenal realism. And it does not yet establish that this is the primary way of defending phenomenal realism. I deal with these issues, in turn, in the remainder of this section.

\subsubsection{Individualist versus Collectivist Way of Appealing to Existence Intuitions}

There would seem to be two different ways in which one might appeal to intuitions that are suggestive of the existence of phenomenal consciousness. In the first way, the philosopher finds the (intuitive) support not in the prevalence of the intuitions but in her own intuitions. Let's call this way of appealing to intuitions the "individualist appeal to intuitions". Alternatively, in the second way, the philosopher may think that the existence of phenomenal consciousness is pretheoretically obvious to almost everyone and this is a good reason to think that phenomenal consciousness does in fact exist. In this way of appealing to intuitions, which we may call "collective appeal to intuitions", the philosopher does not primarily appeal to her own phenomenal intuitions, but rather appeals to the prevalence of intuitions like hers. In other words, she appeals to the wisdom of the crowd. Or more plausibly, the proponent of phenomenal realism may think 
that the prevalence of phenomenal intuitions suggests that they are a natural product of the presence of phenomenal consciousness.

While the examples surveyed above seem suggestive of individualist appeals to intuitions, others might seem to gear more toward a collective appeal. For example, Chalmers (2003, p. 114) clearly states:

The intuition [that phenomenal consciousness is real and poses a hard problem] appears to be shared by the large majority of philosophers, scientists, and others, and it is so strong that to deny it, a type-A materialist needs exceptionally powerful arguments.

And Chalmers (2018, p. 15) repeats this empirical hypothesis by claiming that problem intuitions are widespread or at least have a widely shared basis. In the same vein, Block $(2004$, p. 785$)$ states that the theories against the existence of qualia are inclined "to reject the common-sense conception of subjective experience". 8

While such claims about the phenomenal intuitions of others might be read as appealing to the wisdom of the crowd, I am inclined to think that most philosophers who defend phenomenal realism by claiming that the existence of phenomenal consciousness is pretheoretically obvious are making an individualist appeal. This is because they do not typically claim (at least not clearly) that one should accept that phenomenal consciousness exists because almost everyone has the intuition that phenomenal consciousness exists. However, claiming that consciousness is pretheoretically obvious seems to imply that phenomenal intuitions are prevalent because saying that consciousness is pretheoretically obvious is clearly a more general expression than "it seems to me that phenomenal consciousness exists". Further, even if the philosopher means that phenomenal consciousness is obvious to her, she should naturally expect that the existence of phenomenal consciousness will seem obvious to other people as well unless she suspects that other

\footnotetext{
${ }^{8}$ Block lists these theories as follows: "Opponents of qualia think that the phenomenology of an experience can be exhaustively analysed in terms of its representational or intentional content ("representationism"); or that the phenomenology of experience can be exhaustively analysed in terms of its causal role ("functionalism"), or that having a subjective experiential state can be exhaustively analysed in terms of having a state that is cognitively monitored in a certain way or accompanied by a thought to the effect that I have that state. If we include in the definition of 'qualia' the idea that the phenomenology of experience outruns such intentional, functional and cognitive analyses, then it is controversial whether there are qualia" (Block, 2004, p. 1).
} 
people are cognitively so different from her that their phenomenology notably differs from hers, which we would have little reason to suppose.

In brief, since the realist philosophers usually do not (at least clearly) appeal to the prevalence of existence intuitions to defend phenomenal realism, it is natural to think that they appeal to existence intuitions in the individualist way. However, this way is not completely independent of the prevalence of the existence intuitions because, as noted, if one enjoys an existence intuition, she should expect that her intuition should be shared by other conscious human beings as well. As will be discussed in Chapter 9, this connection between the individualist way of appealing to existence intuitions and the prevalence of these intuitions raises important implications about whether one's appeal to her intuitions provides compelling evidence for phenomenal realism or not if those intuitions are far from being prevalent.

\subsubsection{Why Appealing to Existence Intuitions is the Primary Way of Defending Phenomenal Realism}

We have seen that prominent phenomenal realists often appeal to intuitions to support the existence of phenomenal experiences or qualia. In fact, this is the only clear support offered for phenomenal realism in these works. As Kind (2008, p. 285) writes: "Why should we believe that qualia exist? It would not be surprising if, when confronted with this question, the qualia realist were puzzled." And the reason for this puzzlement is, according to Kind, "because our every experience reveals their existence." And, not surprisingly given this, the result is that phenomenal realists usually give no (further) argument to convince us of the existence of phenomenal consciousness beyond the claim that it is pretheoretically obvious and offering (supposed) examples of phenomenal experiences. To conclude this chapter, I argue that this is no accident: given the standard portrayal of subjective experiences by phenomenal realists, it is unclear what evidence they could offer for the existence of phenomenal experiences beyond such appeals to intuition.

That said, one could still claim that there are arguments for phenomenal realism in which intuitions do not play an essential role. For instance, Robinson (2004)'s defence of phenomenal realism 
based on illusions and afterimages can be considered as being such arguments. ${ }^{9}$ Although I believe that these arguments ultimately rely on a further, specific type of phenomenal intuition-the intuition that sensory properties (specifically colors in these arguments) are properties of our experiences - this might be contested. Yet, it would be far-fetched to claim that phenomenal realism is primarily defended by such arguments considering the rarity of them and given this I'll set the issue of the role of intuitions in such arguments to the side.

Another non-intuition-driven way of defending phenomenal realism would be to appeal to some possible empirical facts that suggest that phenomenal consciousness is real. However, this empirically informed approach seems to be unavailable for phenomenal realism. This is because it seems difficult to find empirical facts suggesting the existence of phenomenal consciousness. Put another way, it seems extremely unrealistic and thus rare to claim that such-and-such brain processes suggest that there is a special type of consciousness (phenomenal consciousness) that cannot be reduced to or that is over and beyond other notions of consciousness such as monitoring consciousness or access consciousness (Block, 2002), which fall under the so-called "easy problems of consciousness" (Chalmers, 1995).

Finally, it is worth noting that Premise 1 of the General Argument does not claim that there are not, or that there cannot be, any non-intuition-driven ways of defending phenomenal realism. Instead, it claims that although there are or might be some realist arguments in which intuitions do not play an essential role, this is not the primary way of defending phenomenal realism. The primary way of defending phenomenal realism is by direct appeal to phenomenal intuitions.

\subsection{Concluding Remarks}

This chapter has provided support for Premise 1 (Phenomenal realism and the epistemic gap thesis are primarily defended by appealing to phenomenal intuitions) with regard to phenomenal realism by giving examples of how prominent phenomenal realists claim that phenomenal realism is pretheoretically obvious, then showing that this claim amounts to asserting that phenomenal

\footnotetext{
${ }^{9}$ Robinson defends the existence of qualia by claiming that illusions give us a good reason to believe that sensory properties belong to our experiences rather than the objects we perceive. He also claims that afterimages suggest that colors are features of our experiences (Robinson, 2004, p. 14).
} 
realism is intuitive (and relatedly, anti-realism is counter-intuitive), and finally showing that such appeals to intuitions are the primary way of defending phenomenal realism. To fully establish Premise 1 of the General Argument, however, I will also need to show that the epistemic gap thesis is primarily defended by appealing to other sorts of phenomenal intuitions, namely epistemic gap intuitions. The next chapter aims to do this. 


\section{THE CENTRAL ROLE OF INTUITIONS IN DEFENCE OF THE EPISTEMIC GAP THESIS}

In the previous chapter, I argued that phenomenal realism is primarily defended by appealing to phenomenal intuitions. For Premise 1 to be fully established, however, I also need to show that the epistemic gap thesis is primarily defended by appealing to intuitions. Doing so is the aim of this chapter.

$\$ 3.1$ will elaborate on the epistemic gap thesis, give a generic definition of the epistemic gap intuition, and explain why I treat phenomenal realism and the epistemic gap thesis as distinct theses. The remaining sections detail the notion that there is an explanatory gap or a hard problem (§3.2), the Knowledge Argument (§3.3) and conceivability arguments (\$3.4), explaining how each relies on a respective type of epistemic gap intuition (explanatory gap intuition, knowledge intuition, and conceivability intuition).

\subsection{The Epistemic Gap Thesis}

The epistemic gap thesis claims that there is an epistemic gap between the physical and the phenomenal. That is, knowing all the physical facts does not suffice to know and explain all the phenomenal facts and does not prevent you from conceiving that physical facts can hold without all the phenomenal facts holding. Supporters of the thesis assert that phenomenal experiences are special in this regard because they claim, there seems no such a gap in other domains. For example, when you know everything physical about lightning, it does not seem that there are still some facts about lightning that cannot be explained in physical terms or that cannot be deduced by the physical facts about lightning. In other words, it does not seem that there is more to lightning to know and explain than electrical discharge in the sky. Further, it seems contradictory and thus inconceivable that when electrical discharge happens, lightning may not happen.

Following Chalmers (2003), the notion of the epistemic gap can be articulated as follows: There is no close epistemic relation between the physical and phenomenal domain. The missing epistemic relation is then held to be one of an a priori entailment- "a relation involving what we can know or conceive or explain" (p. 109). As such, one way to put the claim that there is an epistemic gap is that there is a gap between knowing (or explaining) all of the physical facts and 
knowing (or explaining) all of the phenomenal facts. ${ }^{10}$ Another way to put it is that it is conceivable that a physical copy of our world (or each of us) lacks phenomenal consciousness. Chalmers plausibly lists three major sorts of epistemic gaps, as follows: If $\mathrm{P}$ is the "conjunction of all microphysical facts about the universe" and Q is an "arbitrary phenomenal fact about the universe" (p. 107), then the explanatory gap refers to the idea that $\mathrm{Q}$ is not explainable in terms of $\mathrm{P}$. The knowledge gap refers to the situation in which a perfect reasoner who knows only $\mathrm{P}$ cannot know or deduce that $\mathrm{Q}$. And finally, the conceivability gap refers to one's rationally conceiving of $\mathrm{P}$ without Q (p. 109).

Several clarifications are in order. First, like Chalmers, I take these gaps (knowledge, conceivability, and explanatory gaps) as different forms of the epistemic gap. Knowledge and explanation are clearly enough epistemic terms. As long as whether knowing and explaining a certain set of facts suffice to know and explain another sort of facts is an epistemic issue, whether knowing and explaining all the physical facts is enough to know and explain all the phenomenal facts is an epistemic issue. As for conceivability, as will be seen in the rest of the chapter, conceivability in the relevant sense refers to rationally and coherently conceiving a concept or situation (say a situation in which there are some creatures-philosophical zombies - who are physically identical to us yet lack any capability to feel pain, see colors, and so on). Following the example above, the conceivability of philosophical zombies is an epistemic subject matter because the conceivability of these creatures implies that knowing and considering all the physical facts about humans do not prevent us coherently conceiving them. If there was an a priori connection between physical and phenomenal facts (i.e. if physical facts a priori entailed phenomenal facts), conceiving physical facts without the phenomenal facts would not be possible. So, saying that philosophical zombies are conceivable is another version of saying that there is no a priori connection between the physical facts and phenomenal facts (i.e. there is a conceptual gap between physical concepts/terms and phenomenal terms). Compare conceiving a philosophical zombie with conceiving a married bachelor. Since there is an a priori connection between the concepts of

\footnotetext{
${ }^{10}$ I will use term "fact" in the sense of "true proposition", where a "proposition" is taken as a statement that can be either true or false. By "physical fact", I mean the facts that are defined in the language of physical sciences (physics, chemistry, neuroscience, and so on). Accordingly, "phenomenal fact" refers to any fact about phenomenal consciousness in general or a particular phenomenal state.
} 
unmarried man and bachelor, you cannot coherently imagine a married bachelor! In other words, there is no conceptual gap between the concepts of unmarried man and bachelor. So, coherently conceiving philosophical zombies implies that there is no a priori connection between the phenomenal concepts and physical counterparts. ${ }^{11}$ The supporters of the epistemic gap thesis, whether they be physicalist or anti-physicalist, believe for example that even if we were completely convinced that feeling pain and such-and-such brain state are the same, we could still conceive that brain state without feeling pain because there is a conceptual gap (and no a priori connection) between phenomenal concepts (such as feeling pain) and physical or scientific concepts (such as c-fibers' firing). And so long as whether there is an a priori connection between a certain sort of concepts/facts and the other sort of concepts/facts is an epistemic issue, then the conceivability of a split between the physical and the phenomenal is an epistemic issue.

Second, the epistemic gap thesis does not claim that if a fact is only a posteriori knowable, then there is an epistemic gap between the domain of that fact and the physical domain. ${ }^{12}$ One can point out that we can learn some facts only in an a posteriori or empirical way in the domain of, say, biology but this does not mean that there is an epistemic gap between biology and physical facts. So, why should we assume that learning something about phenomenal consciousness in an a posteriori way indicates that there is an epistemic gap between the phenomenal and the physical? To follow the example above, the empirical gap thesis claims that if some biological phenomena are not entailed a priori by the complete set of physical facts, then there is an epistemic gap between the domain of biology and the physical domain. A biological fact can, of course, be learned a posteriori but if we cannot explain or deduce some biological phenomena from the complete set of physical facts, this means that there is an epistemic gap between biology and physical facts. For example, if the complete physical knowledge still leaves something unexplained about life, or if a

\footnotetext{
${ }^{11}$ Phenomenal concepts are usually thought to be subjective/first person concepts that refer to qualitative character of phenomenal experiences (Balog, 2009). For example, when we introspect on our seeing a red object, phenomenal concepts refer to redness we experience. Phenomenal concepts are contrasted with non-phenomenal material concepts referring to objective/third person non-phenomenal aspects of redness such as reflectance properties of red objects. One can think about redness in both ways: she can think about redness in an objective sense without referring her experience or she can think about her experience itself. The latter way is thought to be involving phenomenal concepts.

${ }^{12}$ I take a posteriori knowledge in the general sense of the knowledge that depends on empirical observations. In this sense of the term, most physical sciences such as biology or chemistry produce and work with a posteriori knowledge while mathematics and logics operate with a priori knowledge which does not depend on empirical observations.
} 
fact about life cannot be deduced from the complete set of physical facts, this would mean that there is an epistemic gap between life and the physical domain. So, the epistemic gap thesis amounts to the thesis that even if we knew all of the physical facts, there would remain some phenomenal facts that should still be explained and/or discovered on an a posteriori basis.

Third, the epistemic gap between the physical and the phenomenal does not mean that there is an ontological gap as well. Note that the epistemic gap concerns what we can know, explain, or conceive rather than what the world is like. We can coherently and rationally conceive for example philosophical zombies, but this does not show that they are genuinely (or metaphysically) possible. So, the epistemic gap alone does not show that physicalism is false because physicalism is an ontological view rather than an epistemic view. It claims that everything is ultimately physical rather than that all the facts can be defined in physical terms and thus can be known or explained by appeal to physical facts (the facts that are defined in terms of physical sciences). So, antiphysicalist philosophers attempt to connect the epistemic gap with the ontological gap in their arguments. For example, as will be seen, the Knowledge Argument, in the most general terms, first makes the epistemic claim that knowing all the physical facts does not suffice to know all the phenomenal facts. Then it claims that if all the facts were grounded in the physical, we would be in a position in which we could know or deduce all the facts (i.e. there would be no epistemic gap), and finally reaches its anti-physicalist conclusion that physicalism is false. The majority of physicalists believe in the existence of the epistemic gap but reject the connection between the epistemic and ontological gap. They believe for example that knowing all the facts is not enough to know or fully explain what it is like to see red and that the philosophical zombies are conceivable. But they reject that this is because what is like to see red involves some non-physical properties, or philosophical zombies are genuinely possible just because they are conceivable. The reason for their rejection of any connection between epistemic and ontological gap is either that epistemic gaps do not entail any ontological gap in general (see for example Block and Stalnaker, 
1999), or that phenomenal consciousness gives rise to an epistemic gap because of the conceptual gap between phenomenal concepts and the physical concepts not because of an ontological gap. ${ }^{13}$

Given the definition and variety of the epistemic gap and clarifications above, now we can define the epistemic gap intuition. It can be said that an epistemic gap intuition is an intuition that reflects a certain sort of denial of epistemic entailment (explaining, knowing, and conceiving) from physical facts to phenomenal facts. That is, an epistemic intuition is the one that reflects that there is a knowledge, explanatory or conceivability gap. In line with this, there are three sorts of epistemic gap intuitions that can be distinguished, with each attaching to a specific kind of epistemic gap between the physical and the phenomenal facts: explanatory, knowledge and conceivability intuitions.

It is important to see that these three sorts of epistemic gap intuitions play a central role in defending the notions of explanatory gap (and relatedly the hard problem) and what Chalmers (2003) calls "epistemic gap arguments". He defines epistemic gap arguments as the arguments that establish first a certain sort of epistemic gap and then conclude that physicalism is false (p. 110). The major examples of these arguments are the Knowledge Argument and conceivability arguments, which relate to knowledge and conceivability gaps respectively and pump the corresponding type of intuition. These arguments include an epistemic premise supported by a certain sort of epistemic intuition (knowledge and conceivability intuitions) and also make the metaphysical claim that there is a further ontological gap between the physical and the phenomenal. As Chalmers puts it, the "conceivability argument infers from conceivability to

\footnotetext{
13 The latter view (known as "Phenomenal Concept Strategy") is the most popular physicalist response to the arguments fuelled by the supposed epistemic gap (among many others, see Balog, 2012; Horgan, 1984; Loar, 1990; Papineau, 2006; Perry, 2001; Tye, 2000). To give an example to how PCS philosophers appeal to phenomenal concepts to explain the epistemic gap, Carruthers and Veillet (2007) explains the conceivability of philosophical zombies and the explanatory gap due to a priori connection between phenomenal and physical concepts as follows: "What is said to be distinctive of such concepts [phenomenal concepts] is that they are conceptually isolated from any other concepts that we possess, lacking any a priori connections with non-phenomenal concepts of any type (and in particular, lacking such connections with any physical, functional or intentional concepts). Given that phenomenal concepts are isolated, the physicalist argues, then it won't be the least bit surprising that we can conceive of zombies and inverts, or that there should be gaps in explanation. This is because no matter how much information one is given in physical, functional or intentional terms, it will always be possible for us intelligibly to think, 'Still, all that might be true, and still this [phenomenal feel] might be absent or different'. There is no need, then, to jump to the anti-physicalist conclusion" (p. 213).
} 
metaphysical possibility; the knowledge argument infers from failure of deducibility to difference in facts" (p. 109).

In the rest of this chapter, I will explain how the notions of explanatory gap (and relatedly the hard problem), the Knowledge Argument and the conceivability arguments appeal to explanatory, knowledge, and conceivability intuitions, respectively. Before doing so, however, it is worth explaining why I treat phenomenal realism and the epistemic gap thesis as distinct theses. Based on the definition of the epistemic gap above, insofar as it is possible that one believes that phenomenal consciousness exists but denies that there is an epistemic gap, there seems a logical space between phenomenal realism and the epistemic gap thesis. A proponent of a functionalist theory about consciousness, for example, may think that the phenomenal character of subjective experiences can be explained by functional processes and properties of mental/brain states so explaining all these functional processes and properties would explain everything about phenomenal consciousness. ${ }^{14}$ It might be argued that functionalist theories explain something else rather than phenomenal consciousness (e.g., Chalmers, 1995, 2003; Frankish, 2012). However, as Chalmers admits, there is still a logical space between the functionalist type of phenomenal realism and the epistemic gap thesis (Chalmers, 2003, p. 112). Further, at least in theory, one could have intuitions that are suggestive of phenomenal realism but lack the intuitions that are suggestive of the epistemic gap thesis. In keeping with this, as will be discussed later, Peressini (2014) claims that laypeople have a conception of qualia but lack the intuition that phenomenal consciousness poses an epistemic gap (or, more specifically, a hard problem).

\subsection{Explanatory Gap Intuition}

The notion of explanatory gap refers to the idea that the explanations cast in terms of physical sciences are not promising to explain a critical feature of experiences: the what-it-is-likeness of

\footnotetext{
${ }^{14}$ Functionalist theories about phenomenal consciousness roughly hold that phenomenal consciousness exists but can be reduced into simpler, "easy" problems such as functional states/properties, representation, perception, and attention. For example, the Global Workspace Theory attempts to explain phenomenal consciousness in terms of functional states which are available for processing by different cognitive systems (Baars, 1993; Dehaene \& Naccache, 2001). Jackendoff (1987) provides an account explaining consciousness in terms of some underlying computational processes. Prinz (2012) attempts to explain phenomenal consciousness in terms of attention which is also an "easy" topic for Chalmers.
} 
them (e.g. what it is like to feel pain, what it is like to see colors, and so on). So, there seems an explanatory gap between physical accounts of experiences and phenomenal aspects of them. The idea that there is an explanatory gap between the physical and the phenomenal is based on a plausible expectation: if nature is a lawful, orderly system that includes only physical stuff, processes and properties, as the physicalists claim, then it should be possible that any phenomena in this system can be explained by the basic, physical principles (Levine, 2001, p. 69). So, physical sciences should give us at least the hope that we can explain phenomenal consciousness in physical terms. However, Levine contends that, unlike other domains, we cannot see how adding more physical knowledge would explain phenomenal experience:

The problem, however, is that there are good reasons for thinking that, unlike other macro domains, when it comes to qualia, we are not lacking merely enough detail to provide the requisite explanation, but any idea of how such a theory might go. That is, there is an explanatory gap between the physical and the mental (at least when it comes to qualia). (Levine, 2001, p. 69)

Levine explains why there is an explanatory gap by contrasting two identity claims: "heat is molecular motion" and "pain is c-fiber firing" where c-fiber firing is just a label for any physical explanation of pain. In the first case, we do not have the intuition that the motion of molecules does not fully explain the heat but in the second case, there seems to be an explanatory gap between pain and c-fiber firing. It seems that any causal, neuroscientific story about pain leaves out the "qualitative character" of pain, i.e. why we feel it in the way we do (Levine, 1983, p. 359). ${ }^{15}$ So, while the reduction of heat to molecular motion is fully explanatory, the reduction of pain to cfiber firing fails to explain (at least) the qualitative character of pain. Likewise, any physical, functional story behind our seeing red seems to leave out why we see red instead of green (Levine, 1983, p. 357).

It is not difficult to see the parallel between the explanatory gap and Chalmers's hard problem. Chalmers (1995) contrasts the easy problems of consciousness with the hard problem of

\footnotetext{
${ }^{15}$ Levine here means how the pain feels by "qualitative character" of pain (Levine, 1983, p. 357) and this can be treated as synonymous with "phenomenal quality" for our purposes.
} 
phenomenal consciousness. Easy problems (such as reportability of mental states, discriminating environmental stimuli, attention, deliberate control of behaviour, and so on) are "easy" because the explanation of the relevant cognitive abilities and functions is sufficient to explain these problems. In other words, it seems that once we explain the relevant cognitive structures, their functions, and relations between them, we have a full explanation of these problems. For example, it is enough to explain how a cognitive system could perform the function of producing reports on internal states to explain reportability. Given an explanation that provides a mechanism for the function at issue or that otherwise provides a functional analysis of reportability, it would be making a conceptual mistake to ask for a further explanation of reportability (p. 203). By contrast, explaining phenomenal consciousness, according to Chalmers, requires more than explaining cognitive functions or mechanisms that are correlated with phenomenal consciousness.

But why exactly does phenomenal consciousness, unlike other types of (non-phenomenal) consciousness - such as access consciousness (Block, 2002) or awareness (Chalmers, 1995) pose a hard problem? Chalmers replies as follows:

What makes the hard problem hard and almost unique is that it goes beyond problems about the performance of functions. To see this, note that even when we have explained the performance of all the cognitive and behavioural functions in the vicinity of experienceperceptual discrimination, categorization, internal access, verbal report-there may still remain a further unanswered question: Why is the performance of these functions accompanied by experience? A simple explanation of the functions leaves this question open. (Chalmers, 1995, p. 203)

It seems clear that the notions of the hard problem and the explanatory gap are closely related if they are not ultimately identical. ${ }^{16}$ They both are based on the same intuition (explanatory gap intuition) reflecting that it seems to us now that even if we were to have a complete story about

\footnotetext{
${ }^{16}$ Chalmers (1995, p. 203) expresses this close relation when he writes: "We know that conscious experience does arise when these functions are performed, but the very fact that it arises is the central mystery. There is an explanatory gap (a term due to Levine 1983) between the functions and experience, and we need an explanatory bridge to cross it". He also explains the notion of explanatory gap in terms of the difference between the easy problems and the hard problem (Chalmers, 2003, p. 105).
} 
phenomenal experiences, we could still ask a non-trivial question: Why and how do the experiences arise and why do we experience them in the way that we do?

Having detailed the notions of the explanatory gap and the hard problem, now we can focus on how exactly these notions are defended by appealing to intuitions. The following subsection will discuss the role of intuitions in defending the existence of the explanatory gap and hard problem.

\subsubsection{The Role of Intuitions in Defending Explanatory Gap and Hard Problem}

It is not difficult to see that the typical support for the explanatory gap comes from the "seems" talk. That is, the answer to the question of why we should think that there is an explanatory gap or hard problem boils down to saying, "because it seems so". Let's take two examples from Levine (1983) introducing the notion of the explanatory gap:

There seems to be nothing about C-fiber firing which makes it naturally "fit" the phenomenal properties of pain, any more than it would fit some other set of phenomenal properties. Unlike its functional role, the identification of the qualitative side of pain with C-fiber firing (or some property of C-fiber firing) leaves the connection between it and what we identify it with completely mysterious. (Levine, 1983, p. 357)

Here, it seems to Levine that no amount of functional explanation of pain could capture the qualitative aspect of pain. And remember that according to our description of intuition, if an idea seems true without any underlying reason, then the idea is an intuitive one for the believer. So insofar as that description is a plausible one, it is also plausible to hold that Levine defends the explanatory gap by appealing to his intuition.

Similarly, when Levine defends the claim that the difference between seeing red and seeing green is not explained by the difference between the physical explanation of seeing red $(\mathrm{R})$ and explanation of seeing green $(\mathrm{G})$, he says:

As evidence for this, note that it seems just as easy to imagine $G$ as it is to imagine $R$ underlying the qualitative experience that is in fact associated with $\mathrm{R}$. The reverse, of course, also seems quite imaginable. (Levine, 1983, p. 358) 
These passages clearly show that Levine's main sort of evidence, if not the only one, for the claim that there is an explanatory gap is his intuitions. In the same vein, Jackson appeals to the explanatory gap intuition in his simple attack on physicalism, although he finds the argument weak from a polemical point of view, as follows: "Nothing you could tell of a physical sort captures the smell of a rose, for instance. Therefore, Physicalism is false. By our lights this is a perfectly good argument. It is obviously not to the point to question its validity, and the premise is intuitively obviously true both to them and to me.” (Jackson, 1982, pp. 127-128).

The same applies to Chalmers's hard problem. As discussed above, Chalmers claims that denying the existence of phenomenal consciousness and the hard problem is counter-intuitive, as follows:

It seems to be a further truth that we are conscious, and this phenomenon seems to pose a further explanandum, one that raises the interesting problems of consciousness. To flatly deny the further truth, or to deny without argument that there is a hard problem of consciousness over and above the easy problems, would be to make a highly counterintuitive claim that begs the important questions. This is not to say that highly counterintuitive claims are always false, but they need to be supported by extremely strong arguments. So the crucial question is whether there are any compelling arguments for the claim that, on reflection, explaining the functions explains everything. (Chalmers, 2003, p. 112)

Here Chalmers clearly asserts that the idea that there is a hard problem over and above the easy problems (that need only functional explanations) is the intuitive one. Thus, rejecting it is so counterintuitive that it needs strong arguments.

Levine, Jackson, and Chalmers appear to follow the individualist way of appealing to intuitions. That is, they think that there is an explanatory gap because it seems to them that there is an explanatory gap, not because they think that the explanatory gap seems obvious to everyone. At least, there is no reason to assume that they base their claims on the prevalence of explanatory gap intuitions. However, there is good reason to think they would predict that the intuition they have is shared by the majority of people. As a matter of fact, as noted in the previous chapter, Chalmers 
makes an empirical claim about the prevalence of the intuitions reflecting that phenomenal consciousness exists and poses an explanatory gap/hard problem:

At a certain point, the debate between type-A materialists and their opponents usually comes down to intuition: most centrally, the intuition that consciousness (in a nonfunctionally defined sense) exists or that there is something that needs to be explained (over and above explaining the functions). This claim does not gain its support from argument but from a sort of observation, along with rebuttal of counterarguments. The intuition appears to be shared by the large majority of philosophers, scientists, and others, and it is so strong that to deny it, a type-A materialist needs exceptionally powerful arguments. (Chalmers, 2003, p. 114)

The empirical claim about the explanatory gap intuition is clear enough here: Not only does Chalmers claim that the large majority of philosophers and scientists have this intuition, but also a large majority of ordinary people. From this passage, it is also clear that Chalmers thinks that the justification for the hard problem (as with phenomenal realism) ultimately rests on the relevant intuitions. Chalmers (2018) repeats the same empirical claim by asserting that the explanatory gap intuition is one of our central intuitions about phenomenal consciousness and asserting that this intuition is widespread.

So, it can be safely asserted that intuitions play a pivotal role in the claim that phenomenal consciousness poses an explanatory gap or a hard problem. After all, in the absence of evidence suggesting that phenomenal experiences have non-physical properties, appealing to explanatory gap intuitions would appear to be the only way to defend that there is an explanatory gap.

\subsection{Knowledge Intuition}

While the explanatory gap intuition concerns the explanatory entailment between the physical and the phenomenal facts, the knowledge intuition concerns the knowledge or deducibility relation between physical and phenomenal facts. The intuition expresses the idea that some phenomenal facts are not included or cannot be deduced from the complete set of physical facts. 
The Knowledge Argument (Jackson, 1982, 1986), which is one of the most famous anti-physicalist arguments, presents a vivid example of this intuition in action. The argument presents a thought experiment in which Mary, who has never seen colors but has the complete set of physical, propositional knowledge about colors, has been prisoned in a black-white room since her birth. The first premise of the argument defines Mary's initial situation: She had all the relevant physical knowledge about seeing colors. The argument, like all the other epistemic gap arguments, relies on a crucial epistemic assumption. The second premise makes such an assumption: when Mary is allowed to leave her room and sees a ripe tomato, she gains new propositional knowledge (in the form of, for example, "that is what it is like to see red" where "that" refers to phenomenal red) upon seeing a ripe tomato. ${ }^{17}$ This suggests that pre-release Mary did not know and could not deduce what it is like to see colors despite her complete physical knowledge about colors. So, the second premise holds that there is a gap between the complete set of physical facts and phenomenal facts. ${ }^{18}$

Following Nagasawa and Stoljar (2004), this second premise reveals what is known as the "knowledge intuition" in the enormous literature concerning the Knowledge Argument (Alter, 2006; Chalmers, 2018; Nida-Rümelin, 2021). Nagasawa and Stoljar define knowledge intuition as follows: "no amount of knowledge of a certain sort-a physical sort-is going by itself to suffice for knowledge of a different sort, namely, a phenomenal sort" (p. 3). Having introduced the

17 "Propositional knowledge" (a.k.a "knowledge-that" or "factual knowledge") can be defined as the knowledge that is presented in the form of propositions such as "color information is stored in V4". In this terminology, physical knowledge is the propositional knowledge that is described in physical terms. Since she has not seen any colors, prerelease Mary's knowledge about colors involves only this type of knowledge. Any defence of the argument is committed to claim that Mary learns this type of knowledge after seeing red because if she gained non-propositional knowledge it would not be plausible to compare her new phenomenal knowledge with her old physical knowledge. For example, if Mary's new knowledge is merely ability knowledge that enables her to recognize and imagine colors, as some philosophers claim in response to the knowledge argument, then there would be no reason to think that her old physical knowledge is insufficient to cover all the facts (true propositions) about the world (Lewis, 1988; Nemirow, 2006).

${ }^{18}$ As with other epistemic gap arguments, the argument proceeds from this epistemic gap to an anti-physicalist conclusion. Jackson concludes that physicalism is false on the grounds that if physicalism was true, pre-release Mary already knew or could deduce the knowledge of what it is like to see red from her complete physical knowledge. Since she could not do this before seeing red, her physical knowledge about colors fails to capture all the facts about colors, which suggests that not all the facts are physical facts and thus physicalism is false. 
Knowledge Argument and defined the knowledge intuition, now we can focus on how this argument appeals to the knowledge intuition, in the next subsection.

\subsubsection{The Role of Knowledge Intuition in the Knowledge Argument}

Given the above definition of knowledge intuition, to see better how Jackson appeals to the knowledge intuition, let's see how the originator of the argument justifies the key and the most controversial premise of the argument, namely Premise 2:

What will happen when Mary is released from her black and white room or is given a color television monitor? Will she learn anything or not? It seems just obvious that she will learn something about the world and our visual experience of it. (Jackson, 1982, p. 130)

Similarly, elsewhere he explains why the second premise is true based on its seeming true:

It seems, however, that Mary does not all there is to know. For when she is let out of the black-white room or given a color television, she will learn what it is like to see something red, say (Jackson, 1986, p. 291).

As can be seen clearly from these passages, "seems" talk plays the central role in the defence of the premise. Why should we believe that Mary learns a phenomenal fact that she did not know in her imprisonment in the black-white room? The only answer available is that because it seems obvious, which makes the premise an intuition-based premise, according to our description of intuitions. I do not find this scandalous because it is natural to defend a claim about a sci-fi case that is impossible to empirically test in our age by appealing to intuitions. So, whether it seems to you that post-release Mary would learn new facts or not (i.e. whether you have the knowledge intuition or not) plays the key role in determining whether or not you believe that post-release Mary would learn new facts about the world.

As in the case of the explanatory gap or hard problem, Jackson and the proponents of the Knowledge Argument, do not seem to defend the second premise by appealing to the prevalence of the knowledge intuition but by appealing to their own intuitions, meaning that they follow the individualist way of appealing to intuitions. As in the case of other types of epistemic gaps, not 
only anti-physicalists but also the majority of physicalists share the knowledge intuition and believe that what the intuition reflects should be taken for granted. To give but one example, Loar holds that the premise reflects an intuitive idea:

The physicalist should accept Jackson's intuitive description of Mary: she fails to know that we have certain color experiences even though she knows all relevant physical facts about us. And when she acquires color experience, she does learn something new about us -- if you like, learns a new fact or truth. (Loar, 1990, p. 598)

However, as with many other examples of individualist appeals to phenomenal intuitions, it is natural for one having the knowledge intuition to predict that the intuition is shared by others as well. And unsurprisingly, the literature abounds with empirical claims about the prevalence of the knowledge intuition. For instance, Nida-Rümelin (2021, 4.7 section, para. 2), when she discusses Tye's reply to the Knowledge Argument, implies that ordinary people share this intuition by saying that "Tye wishes to accommodate the natural intuition that Mary before release cannot fully understand the nature of phenomenal blueness (she doesn't really know what it is to have a blue experience)". And Chalmers offers the same idea more clearly and contends that the knowledge intuition, which he believes is one of our central intuitions about phenomenal consciousness, is shared by non-philosophers as well:

I think the central intuitions are widely shared well beyond philosophy. It is highly plausible that versions of many of these intuitions can be teased out of ordinary subjects, but it is an empirical matter just how widespread they are. (Chalmers, 2018, p. 13)

\subsection{Conceivability Intuitions}

A conceivability intuition, in the most general terms, reflects that the physical and the phenomenal may split from each other. What the intuitions exactly reflect depends on the definition of conceivability. Following Chalmers (2010, p. 144), a proposition or statement (S) is negatively conceivable when it cannot be ruled out through a priori reasoning" and "S is positively conceivable when one can coherently imagine a situation in which $\mathrm{S}$ is the case". As such, one's conceivability intuition may express the negative idea that the existence of a world in which the phenomenal and the physical are not identical, or in which the phenomenal can change without 
any change in the physical, cannot be ruled out through a priori reasoning. For example, if it seems to one that there is no contradiction in the notion of a philosophical zombie - a creature that is physically identical to us but lacks phenomenal consciousness-or in the proposition that philosophical zombies exist, then that person has a conceivability intuition in this negative sense. In the positive sense of conceivability, a conceivability intuition reflects the idea that one can coherently imagine a scenario in which the phenomenal and the physical pull apart from each other. As such, for example, if one can coherently imagine a world inhabited by philosophical zombies, or that the proposition that philosophical zombies exist is verified, then that person has a conceivability intuition in the positive sense.

Conceivability arguments are the arguments premised on the conceivability intuition. From Locke (1689/1975) to Block (1990), Kripke (1980) and Chalmers (1996, 2009), these arguments have been frequently appealed to by anti-physicalists. ${ }^{19}$ They aim to draw out the conceivability intuition by presenting different scenarios in which the phenomenal and the physical splits and suggest that the conceivability of such scenarios is a reliable guide to the genuine possibility of the split in question. These arguments have an epistemic premise that pumps the conceivability intuition. In Locke's and Block's inverted spectrum cases, it is claimed that we may enjoy systematically different phenomenal properties despite the same physical conditions. That is, it is asserted that it is conceivable that you could see red while I see green when we look at the grass, for instance, even if we have exactly the same visual capabilities and all the other relevant physical conditions are the same. In Kripke's and Chalmers's arguments, the conceivability intuition is drawn out in terms of our physical duplicates (philosophical zombies in Chalmers's terminology) living in a physically identical world that lacks phenomenal consciousness altogether. Goff (2012)'s notion of Ghosts-phenomenal duplicates lacking a body-is the opposite of philosophical zombies but reflects the same conceivability intuition.

19 Descartes's sixth meditation (Cottingham, Stoothoff, Murdoch, \& Kenny, 1985) is sometimes also taken as presenting a conceivability argument. However, Descartes, problematizes the identity between the mind and the body in general (rather than specifically the relationship between the phenomenal states and the physical states) and holds that we can conceive unembodied minds. For the purpose of this thesis, I will concern only the conceivability arguments that specifically question the identity or supervenience relation between the physical and the phenomenal. 
Unlike the explanatory gap and the knowledge intuition, the connection between the epistemic gap and conceivability intuition is not straightforward. Explanatory gap and knowledge intuitions clearly and straightforwardly suggest an epistemic gap: the former intuition reflects that physical knowledge does not suffice to explain phenomenal consciousness and the latter intuition reflects that not all the phenomenal facts can be deduced by the complete set of physical facts. But why should we consider conceivability as an epistemic matter in the first place? After all, one who knows all the relevant details about a conceived scenario might still be unable to reflect on all the implications of her complete knowledge because of some cognitive limitations. Most people, for example, cannot rule out the falsity of a very complex mathematical fact. In such a case, the conceivability of the falsity of this complex mathematical fact would be only prima facie conceivability (Chalmers, 2009, p. 315). As such, this would not suggest anything that is of epistemic significance. ${ }^{20}$ Similarly, the conceiver of a zombie-world may be unable to rule out the existence of philosophical zombies on an a priori basis upon initial consideration because of the limitations of the brain, in which case there would be no reason to think that there is an epistemic gap between the phenomenal and the physical. That is, this would show that we are unable to see whether a philosophical zombie is possible or not (because we would not be able to understand whether such a possibility contradicts all the implications of the physical facts) rather than that philosophical zombies are possible in the ideal cognitive conditions and thus that there is an epistemic gap between physical facts and the phenomenal ones.

So, conceivability arguments should appeal to ideal conceivability rather than prima facie conceivability to suggest an epistemic gap between the phenomenal and the physical. Chalmers defines ideal conceivability as follows: "S is ideally conceivable when the hypothesis expressed by S cannot be ruled out a priori even on ideal rational reflection" (Chalmers, 2010, p. 143). This way, ideal conceivability abstracts away any cognitive limitation. However, how can one ideally conceive anything given our cognitive limitations? Chalmers seems to think that despite our limitations, the (supposed) conceivability of philosophical zombies is suggestive of their ideal

\footnotetext{
${ }^{20}$ Chalmers defines prima facie conceivability as follows: "We can say that $\mathrm{S}$ is prima facie conceivable (for a subject) when that subject is unable to rule out the hypothesis expressed by $\mathrm{S}$ by a priori reasoning on initial consideration" (Chalmers, 2010, p. 143).
} 
conceivability. ${ }^{21}$ As such, he attempts to shift the burden of proof to his opponents by saying that to reject the ideal conceivability of a split between the phenomenal and the physical (or simply philosophical zombies), "one needs to find something that undermines the prima facie coherence and imaginability, such as some sort of a priori incoherence, contradiction, or unimaginability in the hypothesis that emerges on reflection" (Chalmers, 2010, p. 154). So, Chalmers believes that, despite our cognitive limitations, the ideal conceivability of philosophical zombies can be defended by relying on our prima facie conceivability intuitions because they suggest ideal conceivability of a split between the phenomenal and the physical, which in turn suggests an epistemic gap between these realms.

The claim that the (supposed) prima facie conceivability of zombies is a good reason to assume that they are also ideally conceivable is highly controversial. This controversy is beyond the scope of the present thesis, however. What is more relevant is that prima facie intuitions concerning philosophical zombies (or any other split cases) are needed to defend and to provide at least defeasible support for the idea that they would be conceivable even for an ideal reasoner. And if split cases are ideally conceivable, this shows that there is an epistemic gap. ${ }^{22}$ This is because, if physical facts implied all the phenomenal facts (i.e. if there was no epistemic gap between the

${ }^{21}$ Against the objection that zombies are prima facie conceivable but not ideally conceivable because prima facie epistemic gap between phenomenal and physical truths can be closed by ideal reflection because of new discoveries in science, Chalmers (2010, p. 155) says that "to render zombies incoherent even on ideal reflection requires some sort of conceptual link between physical and phenomenal concepts. Given that physics and physical concepts are all structural-dynamic in character (and new scientific developments are unlikely to change this, although see option 3), phenomenal concepts must have a character that is linked to structural-dynamic concepts in an appropriate way. Upon examination, the only candidate that is remotely tenable is the hypothesis that phenomenal concepts are functional concepts, but we have already seen that there is good reason to reject that view". And against the objection that "nonideal creatures such as ourselves cannot know whether or not a given statement is ideally conceivable", he states that "Although we are nonideal, we can know that it is not ideally conceivable that $0=1$ and that it is ideally conceivable that someone exists. We know that certain things about the world (say, that all philosophers are philosophers) are knowable a priori and that certain things about the world (say, that there is a table in this room) are not so knowable even by an ideal reasoner. Likewise, reasoning of this sort gives us very good reason to think that there is no a priori entailment from physical to phenomenal truths and that zombie hypotheses are conceivable even for an ideal reasoner". ${ }^{22}$ It can be said that the conceivability intuition in its full sense (that requires one to ideally conceive the split between the phenomenal and the physical) comes down to the deducibility intuition because it implies that the falsity of any split between the phenomenal and the physical cannot be deduced by the complete set of physical facts. Or, it can be said that, the conceivability intuition comes down to the explanatory gap intuition in that if the physical story could explain phenomenal consciousness, any split between the phenomenal and the physical would not be ideally conceivable. Levine (2001), for example, claims that "if the physical story really explained the qualitative character, it would not be so clearly imaginable that the qualia should be missing" (p. 79). Note that in both views ideal conceivability implies an epistemic gap between the physical and the phenomenal. 
phenomenal and the physical), one would not be able to ideally conceive the split scenarios because ideal reflection on those scenarios - together with the consideration of the implications of the complete physical knowledge- would reveal some contradictions in them.

Having established the connection between conceivability intuitions and the epistemic gap, we can now focus on how the conceivability intuitions are employed in the conceivability arguments. The next section will attempt to do this.

\subsubsection{The Role of Conceivability Intuitions in the Conceivability Arguments}

To show that how conceivability intuitions play a central role in the conceivability arguments, I will give just two examples, one from Kripke and one from Chalmers, but the central role of intuitions in other conceivability arguments can be established similarly.

Kripke defends the conceivability (in the sense of logical possibility) of the split between the phenomenal and the physical as follows:

Let ' $\mathrm{A}$ ' name a particular pain sensation, and let ' $\mathrm{B}$ ' name the corresponding brain state, or the brain state some identity theorist wishes to identify with A. Prima Facie, it would seem that it is at least logically possible that B should have existed (Jones's brain could have been in exactly that state at the time in question) without Jones feeling any pain at all, and thus without the presence of A. (Kripke, 1980, p. 146)

Similarly, he asserts that identification of mental states with brain states seems contingent (i.e. possibility of their split) to us:

The correspondence between a brain state and a mental state seems to have a certain obvious element of contingency. (p. 154)

Chalmers more explicitly reveals how he defends the conceivability gap between the physical and the phenomenal by noting that assertion of the conceivability of philosophical zombies comes down to the brute intuition that the zombie-world can be conceived coherently, as follows: 
I confess that the logical possibility of zombies seems equally obvious to me. A zombie is just something physically identical to me, but which has no conscious experience-all is dark inside. While this is probably empirically impossible, it certainly seems that a coherent situation is described; I can discern no contradiction in the description. In some ways an assertion of this logical possibility comes down to a brute intuition, but no more so than with the unicycle. (Chalmers, 1996, p. 96)

As seen, both philosophers back up the idea that a split between the physical and the phenomenal is (negatively) conceivable by basically claiming that the conceivability of such a split seems obvious to them. Given the proposed description of intuitions, which consider this type of "intellectual seemings" as intuitions, it is clear that these philosophers appeal to conceivability intuitions while defending their (conceivability) arguments. In line with this, Chalmers notes how defences of conceivability arguments (inverted spectrum and his zombie argument) — and the Knowledge Argument-ultimately rest on intuitions:

It might be further objected that the arguments I have given consist, at bottom, in a collection of intuitions. There is certainly a sense in which all these arguments are based on intuition, but I have tried to make clear just how natural and plain these intuitions are, and how forced it is to deny them. (Chalmers, 1996, p. 110)

It seems also clear they appeal to the conceivability intuition in the individualist way because they do not base their defence on the prevalence of conceivability intuitions. But again, it is natural to predict for one having a conceivability intuition that other people also share her intuition. So, it is no wonder that Chalmers claims that almost everybody is capable of conceiving of the possibility that philosophical zombies may exist and thus that the burden of proof lies on those who disbelieve the logical possibility of philosophical zombies, as follows.

Almost everybody, it seems to me, is capable of conceiving of this possibility. Some may be led to deny the possibility in order to make some theory come out right, but the justification of such theories should ride on the question of possibility, rather than the other 
way around. In general, a certain burden of proof lies on those who claim that a given description is logically impossible. (Chalmers, 1996, p. 96) ${ }^{23}$

Chalmers (2018) also believes that the conceivability intuition (modal intuition in his terminology) is one of our central intuitions about consciousness (p. 12) which he assumes are widely shared or "at least they have a widely shared basis" (p. 15).

\subsection{Concluding Remarks}

To summarize, in this chapter I've argued that the main, if not the only, way to defend the epistemic gap thesis is to pump epistemic gap intuitions, viz. explanatory, knowledge or conceivability intuitions. We have seen that the notion of the explanatory gap, the Knowledge Argument and the conceivability arguments assume a certain sort of epistemic gap which is defended by simply claiming that what is assumed seems obvious without giving any underlying reason, that is why I and many others consider this claim as resting on intuitions. We have also seen that this individualist way of appealing to intuitions is sometimes accompanied by empirical claims about the prevalence of the epistemic gap intuition in question. Coupled with the previous chapter, I have made the case for Premise 1 of the General Argument: Phenomenal realism and the epistemic gap thesis are primarily defended by appealing to intuitions. Chapters 4-8 will now defend Premise 2 (phenomenal intuitions are not prevalent), with Chapters 4-6 investigating the prevalence of existence intuitions while Chapters 7-8 investigate the prevalence of epistemic gap intuitions.

${ }^{23}$ Chalmers is not alone in thinking that conceivability intuitions are widespread. The majority of physicalist philosophers also believe that what Dennett (2001) calls "Zombic Hunch" is widespread. To give but an example, Papineau (2007), claims that Kripke's conceivability argument relies on the observation that the mind-brain identity seems contingent even to strong believers such as physicalists (p. 483), and holds that this observation is true. He goes on with claiming that the resistance to mind-brain identity is not because of the fact that our knowledge about the brain is infant and thus physicalism lacks conclusive evidence (p. 491), and makes an empirical claim about the prevalence of the conceivability intuition, as follows:

"Imagine that physicalist scientists have now uncovered overwhelming evidence that in the actual world pain always goes hand in hand with some brain process K. Wouldn't it still strike them that there could be a possible being with process K yet no pain? I would have thought that this intuition would remain" (p. 492). 


\section{PREVALENCE OF EXISTENCE INTUITIONS IN THE LITERATURE}

In the previous two chapters, I argued that the first premise of the General Argument (that phenomenal realism and the epistemic gap thesis are primarily defended by appealing to intuitions) is plausible by way of noting the central role of existence and epistemic gap intuitions in defending phenomenal realism and the epistemic gap thesis, respectively. The second premise of the General Argument holds that phenomenal intuitions - the intuitions used to defend phenomenal realism and the epistemic gap thesis - are not prevalent. As with the first premise of the argument, this premise needs to be defended with respect to both phenomenal theses. As such, Premise 2 can be divided into two sub-premises:

Premise 2-1: Existence intuitions are not prevalent.

Premise 2-2: Epistemic gap intuitions are not prevalent.

This and the next two chapters aim to establish Premise 2-1. In the second chapter, we saw that phenomenal realism is justified by appealing to existence intuitions. We also saw that the proponents of phenomenal realism sometimes implicitly or explicitly claim that existence intuitions are shared by laypeople and, thus, that phenomenal consciousness is not a philosophical fiction. However, an immediate question arises: How could an ordinary person have existence intuitions, considering that "phenomenal consciousness" is a technical phrase? It is important to see at this point that one may have some intuitions that individually or collectively suggest that there are phenomenal experiences even if one does not recognize such technical terms and phrases as "phenomenal consciousness" and "qualia". One may, for example, have the intuition that colors are qualities of our visual experiences rather than the objects we see or the light bouncing off those objects, or that pain is the quality of our experience of feeling pain rather than the parts of the body that are felt to hurt. That is, in a general form, one may have the intuition that sensory qualities are properties of our experiences, i.e. that they are qualities of mental states. I will call this type of intuition "experiential property intuitions".

Another type of existence intuition reflects that there is something that it is like to be in some mental states (seeing colors, feeling pain and so on) but not others (believing, knowing, making inferences and so on), and relatedly that some entities (such as humans, dogs, bats and other complex animals) but not others (artefacts such as calculators, thermostats, simple robots and so 
on) can enjoy these states. For example, one may have the intuition that a simple robot cannot feel pain, see colors, enjoy music, etc., while having the intuition that it can believe something, desire to do something and so on. That is, one may have intuitions that suggest that an entity can enjoy non-phenomenal experiences but cannot enjoy the phenomenal ones. I will call this type of intuition "common aspect intuitions".

Of course, there might be other sorts of existence intuitions. For instance, there are what Chalmers (2018, p. 12) terms "metaphysical intuitions"-intuitions that colors, pains and so on are nonphysical qualities. However, I hold that the experiential property and common aspect intuitions are the core intuitions that would indicate that one tends to believe that phenomenal consciousness exists and, relatedly, that would suggest that a person has a conception of phenomenality, whether tacit or explicit. And reversely, if one did not enjoy these intuitions, it would be difficult to argue that one has a conception of phenomenality. Note that, we cannot say this for metaphysical intuitions, since it is not essential to phenomenal realism that phenomenal qualities are nonphysical. So, one may lack this intuition but still have intuitions that are suggestive of phenomenal realism.

Based on these considerations, we can say that there are two key targets for testing whether laypeople have existence intuitions: experiential property intuitions and common aspect intuitions. That is, testing whether laypeople have one of or both these two sorts of intuitions would give us a good estimate of the prevalence of existence intuitions. Fortunately, there are already experimental studies testing the prevalence of these intuitions.

In this chapter, I will discuss these studies, focusing on whether they indicate that existence intuitions are prevalent or not. $\S 4.1$ will discuss studies bearing on the prevalence of experiential property intuitions. I will claim that these studies strongly suggest that experiential property intuitions are not widespread and will reply to some possible objections. $\$ 4.2$ will discuss studies bearing on the prevalence of common aspect intuitions, including studies from Knobe and Prinz (2008; KP) and Sytsma and Machery (2010; SM). I will claim in $\$ 4.2 .1$ that, pace the authors, the studies in KP do not provide compelling evidence for the prevalence of common aspect intuitions. In $\S 4.2 .2$, I will argue that SM provides more compelling evidence pointing in the opposite direction of KP. However, SM also faces some objections. Based on the results of his second study, 
Peressini (2014) claims that there is a lay conception of phenomenal consciousness, although it differs from the philosophical conception. $\$ 4.2 .3$ will discuss this study and contend that Peressini's findings can reasonably be interpreted in an alternative way and do not provide compelling evidence for the existence of a folk conception of phenomenal consciousness. In the same way, the findings in SM have also been interpreted by the critics differently from Sytsma and Machery. $\$ 4.3$ will reply to some possible objections to SM, focusing on the most common objection to SM's conclusion, namely the Ambiguity Objection.

\subsection{Prevalence of Experiential Property Intuitions}

I noted in Chapter 2 that the idea that the properties we are acquainted with in perceptual states and bodily sensations are the properties of mental states is pivotal to phenomenal realism. As such, if phenomenal consciousness is pretheoretically obvious, then the vast majority of people should tend to locate the properties we are acquainted with in prototypical "phenomenal" cases in the mind rather than the perceived object or light (in cases like seeing colors) or body parts (in cases like feeling pain). Relatedly, they should think that there cannot be any pain without the person feeling it because they think that painfulness is a feature of experiences of feeling pain. However, the studies that will be discussed in this section suggest the reverse, indicating that laypeople, by and large, lack experiential property intuitions.

\subsubsection{Where are Colors and Pains?}

Firstly, let's see where laypeople locate colors and pains. Sytsma (2010) questions the accuracy of the claim that laypeople treat the qualities that we experience in ordinary perception as the qualities of mental states. In his first study participants were given a brief prompt followed by four questions:

There is an old puzzle that many people are familiar with: "If a tree falls in the woods and no one is there to hear it, does it make a sound?" Philosophers have posed a similar question about vision: "If there was nobody there to see it, would a ripe tomato still be red?" Some philosophers have argued that tomatoes (and other objects) are not really colored, rather the red is produced in your mind when you look at the otherwise uncolored tomato. Other philosophers have disagreed, arguing that the tomato itself is truly red-that the red that 
we see is the red of the tomato. We are not interested in which of these positions is "correct" (or even if there is a correct answer to these questions). What we want to know is how you think about colors-we want to know your intuitions about these questions.

1. Do you think that a ripe tomato would still be red even if there was nobody around to see it?

2. Do you think that the red you see when you look at a ripe tomato is in your mind?

3. Do you think that the red you see when you look at a ripe tomato is in the tomato?

4. Do you think it is possible that somebody else might actually see the color that you call "blue" when they look at an ordinary ripe tomato, despite having normal visual acuity (i.e., without being color-blind)? (p. 120)

The first three questions are clearly related to the question of whether colors belong to the mind or objects. As for the fourth question, it tests whether laypeople think that inverted spectrum cases are possible or not. ${ }^{24}$ Inverted spectrum cases describe a situation in which two people with normal visual capabilities see colors systematically differently from each other when they look at the same object, even though both discriminate between colors with equal accuracy and make the same verbal judgements about colored objects. The supposed intuition for such cases is that we cannot be sure that two people see the same color while looking at the same object in the same physical conditions even if they have the same visual capabilities. This situation is standardly held to be possible only if colors are properties of experiences rather than the properties of the objects outside the skull because if they belong to an external object(s), there would be no reason to expect that two perceivers with identical visual capabilities would see different colors when they look at the same object.

\footnotetext{
${ }^{24}$ Inverted spectrum cases or inverted qualia cases in general (see Locke (1689/1975) and Block (1990) for the most known examples) have been used not only for the existence of experiential properties but also, as noted in Chapter 3 , for the conceivability of a split between the phenomenal and the physical, as well as for the claim that experiential properties are not functional or representational. See Byrne (2020) for examples of appeals to inverted qualia cases for different purposes. In $\S 7.1 .1$, I will discuss the same question in terms of the prevalence of conceivability intuitions.
} 
If laypeople, by and large, had experiential property intuitions we would expect to see high responses for Questions 2 and 4 in Sytsma's study, but low responses for Questions 1 and 3. Sytsma's results, however, were the opposite: The respondents tended to believe that colors are the features of the objects outside the mind rather than features of mental states, and that inverted spectrum cases are not possible.

Similar results were repeated not only for colors but also for pain in Sytsma's second study. The participants in this study tended to believe that "the red is really in the tomato and is simply seen by the mind" rather than that "the red is produced in your mind and is merely caused by the tomato" (p. 122). They also tended to endorse that "the pain is really in the injured toe and is simply felt by the mind" rather than "the pain is produced in your mind and is merely caused by the injured toe" (p. 122). As in the first study, participants, by and large, held that ripe tomatoes would be red even if there were no people to see it, that the redness is in the tomato, and that the redness is not in the mind. In parallel, participants tended to hold that "there is still pain in a badly injured leg even when the person is not aware of it" (p. 123), that the pain they feel when they forcefully stub their toes is in the toe, and that the pain they feel is not in their minds.

Studies 5 and 6 in Sytsma (2010) tested a further prediction based on the results of his second study: if laypeople, by and large, locate pains in the objects outside the mind, then they should tend to believe that pain can be shared if the afflicted body part is shared. Study 5 compared responses for a "three-legged race" scenario in which the legs of two racers are tied together, their common "third" leg is injured, and both racers grimace and shout out "Ouch!" to responses for a "conjoined twins" scenario in which the independent racers are replaced with a pair of twins who share the lower part of their bodies. Participants were asked whether the people in these scenarios felt one and the same pain or two different pains. As expected, participants tended to believe that the racers in the "three-legged race" scenario felt different pains. In contrast, participants gave low responses to the case in which the same injured body part is shared. ${ }^{25}$ And similar results were

\footnotetext{
25 The vignettes were as follows:
} 
found for an alternative vignette in Study 6 involving two people surgically attached to the same donor hand by a mad scientist. In brief, these two studies suggest that laypeople tend to think that people can share pains, which would be impossible if they thought that pains are a feature of our private, mental life.

Further, Kim, Poth, Reuter, and Sytsma (2016) found similar results to Sytsma (2010). They conducted two cross-cultural studies where they asked American and South Korean participants about the location of pain. The majority of participants for each group located the pain in the body while only a small minority located pain in the mind.

\subsubsection{Unfelt Pain}

The belief that pain and feeling pain are the same is common in philosophical circles. ${ }^{26}$ In other words, philosophers standardly believe that if you don't feel pain, there is no pain, which amounts to saying that if there is no painful experience, then there is no pain. Note that if laypeople believe that painfulness depends on the existence of painful experiences (i.e. that painfulness belongs to the mental states), then they would tend to think that there cannot be any pain if one does not experience it. Study 4 in Sytsma (2010) investigated whether laypeople share this belief or not. Participants were given the following vignette:

Doctors have observed that sometimes a patient who has been badly injured will get wrapped up in an interesting conversation, an intense movie, or a good book. Afterwards, the person will often report that during that period of time they hadn't been aware of any

Henry and Johnny are normal undergraduates at a state university. They are distinct people with their own beliefs and desires. One day they were participating in a three-legged race in a park with Henry's right leg tied to Johnny's left leg. While running toward the finish line their 'third-leg' forcefully kicked a large rock that, unbeknownst to them, was hidden in the grass. Henry and Johnny both grimaced and shouted out 'Ouch!'.

Bobby and Robby are conjoined twins that are joined at the torso. While they are distinct people, each with their own beliefs and desires, they share the lower half of their body. One day while running through a park they forcefully kicked a large rock that, unbeknownst to them, was hidden in the grass. Bobby and Robby both grimaced and shouted out 'Ouch!' (p. 126).

On the 1-7 scale, the mean for three-legged scenario and conjoined twin scenarios were 5.40 and 3.29 , respectively.

${ }^{26}$ Reuter and Sytsma (2018) notes that most philosophers believe that the existence of pain depends on its being felt and gives many examples of philosophers sharing this idea. 
pain. In such a situation, do you think that the injured person still had the pain and was just not feeling it during that period? Or, do you think that there was no pain during that period? (p. 125)

The mean response to the question was significantly below the neutral point, which suggests that laypeople by and large have the intuition that unfelt pain is possible, which in turn suggests that they do not take pain to be a feature of feeling pain.

Reuter and Sytsma (2018) found similar results. Their first study replicated Sytsma's distracted patient case. Reuter and Sytsma found that $90.3 \%$ of participants believe that when she was distracted, the injured person still had the pain and was just not feeling it during that period. They conducted other studies suggesting that laypeople tend to believe that one can still be in pain even if one does not feel it because of painkillers (studies 2-5) or being distracted by the life and death struggle during a war (studies 6-9). Study 10 addressed the objection that participants might have thought that if one feels pain before or after something that prevents her from feeling pain (such as taking painkillers), then there must be pain even when she did not feel it. The study describes a situation in which one goes through an injury but never feels it: a soldier called Greg who has a terrible injury on his left leg reports to a medic that he feels no pain and dies shortly after the medic examines him. In contrast to what we would expect if the objection was correct, $67.0 \%$ of participants chose the statement that "when the field medic was examining his injury, Greg had pain but didn't feel it" over "when the field medic was examining his injury, Greg had no pain". Lastly, Studies 11-17 involved direct questions about whether all, most, some, or no pains are felt in different ways. Across these studies, only a minority of participants indicated that all pains are felt. For instance, in Study 15, Reuter and Sytsma asked, "Is it possible for a person to have a pain that they don't feel for a period of time?" $92.2 \%$ of the participants answered affirmatively.

In sum, the studies reviewed in this section suggest that laypeople by and large locate colors and pains outside the brain and do not treat them as properties of mental states. The folk conception of 
pain seems to treat pains as bodily states rather than mental states and, as such, is at odds with a corollary of the phenomenal conception of pain - that pain is feeling pain. ${ }^{27}$

It might be objected, however, that these studies do not rule out the possibility that although laypeople believe that colors and pains are located outside the mind, they also think that the experiences of seeing colors and feeling pain have experiential properties. For example, it is possible for one to think both that colors belong to external objects and that there are properties of the experience of seeing colors, e.g. the properties that characterize what it is like to see a given color. Similarly, one can think both that there can be pain in the absence of feeling pain and that feeling pain itself has some properties, e.g. the properties characterizing what it is like to feel pain. Surely, this is possible. That said, however, there seems to be no reason to think that our perceptual experiences reveal something that is above and beyond the properties of the object(s) outside the mind. Indeed, when I try to find some properties of my experiences rather than the properties of the objects that I perceive, I always fail. This failure, which I believe is common, is often referred to in terms of the "transparency" or "diaphanousness" of experiences in the literature. ${ }^{28}$ But, for the sake of the objection, let's assume that laypeople do not share the "transparency intuition" (Kind, 2003) and tend to believe that experiences themselves have some properties characterizing what it is like to undergo the experience. Even so, it seems far-fetched to think that some experiential properties over and above the colors seen characterize what it is like to see colors, or that some experiential properties over and above the properties of pain characterize what it is like to feel pain. To put the point differently, there may be some properties of experiences in addition to, say, colorful properties of the external objects but there seems no reason to assume that these "colorless" properties contribute to the colorfulness of experience. At least, it seems ad hoc to

\footnotetext{
27 There are also some studies suggesting that laypeople tend to think that pain and feeling pain are different things from the reverse direction. Reuter, Phillips, and Sytsma (2014) and Sytsma and Reuter (2017) suggest that laypeople mostly think that there can be a feeling of pain without a pain (i.e. pain hallucinations).

${ }^{28}$ Harman (1990, p. 667) explains the transparency of experience in a famous passage: "When Eloise sees a tree before her, the colors she experiences are all experienced as features of the tree and its surroundings. None of them are experienced as intrinsic features of her experience. Nor does she experience any features of anything as intrinsic features of her experiences. And that is true of you too. There is nothing special about Eloise's visual experience. When you see a tree, you do not experience any features as intrinsic features of your experience. Look at a tree and try to turn your attention to intrinsic features of your visual experience. I predict you will find that the only features there to turn your attention to will be features of the presented tree".
} 
expect one who believes that colors/pains belong to the external world to ascribe some properties to experience that contribute to the colorfulness/painfulness of the colorful/painful experience.

Alternatively, one might contend that even if one believes that pain can exist without being felt, she could still believe that the experience of pain, rather than the pain, has the property of painfulness on the grounds that an unfelt pain cannot be painful. After all, how can the pain in my leg be painful if I do not feel it? But this is just another way of making the same claim that laypeople seem to resist: there can be pain only if it is felt. If one thinks that a ripe tomato can still be red even if we do not see it, we would expect the same person also to believe that the tomato, rather than our experience of seeing it, has the redness. And it is natural to assume that the same holds for pain: If one thinks that pain is not in the mind, and that there can be pain without that pain being felt, we would expect her also to believe that there might be some painful bodily damage (i.e. the damage that has painful properties) even if we are not aware of them.

In this section, we have seen that the laypeople differ from most philosophers in locating paradigmatic "phenomenal" properties (colors and pains) in the objects outside the mind. That is, laypeople by and large seem to lack experiential property intuitions. The next section will investigate the prevalence of the other main type of existence intuitions, namely common aspect intuitions.

\subsection{Prevalence of Common Aspect Intuitions}

As noted in Chapter 2, it is commonly believed in philosophical circles that phenomenal consciousness is one of the most obvious mental phenomena, even for laypeople. As such, we should expect laypeople to (tacitly) recognize an important common aspect between some experiences, such as feeling pain and seeing colors; that is, that there is something that it is like to undergo each of these types of experiences, such that they have something important in common. In other words, we would expect laypeople to have common aspect intuition. The basic idea is that laypeople might be thought to have a tacit conception of phenomenality if they recognize there as being a common, qualitative aspect shared by different experiences, just as a child who does not know what "triangle" means could nonetheless be considered to have a tacit conception of 
triangularity if she recognizes the commonality between equilateral, scalene, and right-angled triangles.

Let's call this the "common aspect claim"- the claim that laypeople recognize the (supposed) qualitative aspect common to different phenomenal states. If the claim is true, laypeople should happily ascribe phenomenal states to an experiencer (an entity that is usually thought by philosophers to be phenomenally conscious such as a human or a dolphin). On the other hand, they should deny those states of non-experiencers (entities that are usually thought to lack phenomenal consciousness such as simple robots) while, potentially, being more willing to attribute some nonphenomenal states (such as believing, detecting, and so on) to them.

As such, one of the most straightforward ways to assess the common aspect claim is to investigate lay attributions of different mental states to experiencers and non-experiencers, and specifically those states that philosophers tend to think of as phenomenal versus non-phenomenal. If these attributions divide along lines of phenomenality, then this would suggest in favour of the common aspect claim. For example, if people denied attributing feeling pain and seeing colors to a simple robot while happily attributing believing and knowing to it, this would at least cohere with the claim that laypeople recognize the common aspect between feeling pain and seeing colors. On the other hand, if they did not, this would cast considerable doubt on the claim because it would suggest that laypeople don't recognize the common, qualitative aspect of these states, assuming that like philosophers they don't consider a simple robot to be an experiencer.

The second study in KP and the first study in SM test the common aspect claim in this way, asking participants about attributions for phenomenal and non-phenomenal mental states, although they arrive at different conclusions. Knobe and Prinz hold that the results of their study suggest that laypeople share a tacit conception of phenomenal consciousness, whereas Sytsma and Machery found opposing results that suggest against the common aspect claim.

\$4.2.1 will discuss KP and note that the results in this work can be explained in alternative ways that have nothing to do with phenomenal consciousness. So, I will conclude that this study does not provide strong evidence for the common aspect claim. On the contrary, SM provides strong 
evidence against the common aspect claim. §4.2.2 will explain how SM conclude that laypeople do not have a conception of phenomenal consciousness.

A second way to assess the common aspect claim is to ask participants some independent and specific questions about so-called phenomenal states and look at whether the majority of answers matches the answer that we would expect from them if they recognize the (supposed) phenomenality of those states. Peressini (2014), for example, used this type of approach and argues for something like the common aspect claim, although he believes that the lay conception of phenomenal consciousness differs from the standard, philosophical conception of phenomenality that gives rise to the hard problem. I will discuss Peressini’s study in $\$ 4.2 .3$ and claim that it does not present compelling evidence for the common aspect claim and relatedly does not provide a convincing challenge to SM's conclusion. However, SM's conclusion has been criticized in the literature, mostly in the form of the Ambiguity Objection, as I will discuss in $§ 4.3$.

\subsubsection{Knobe and Prinz's Study}

Knobe and Prinz presented some of the first studies, if not the first studies, to investigate the common aspect claim. The results of their studies, especially their second study, seem to provide positive evidence for the claim. However, it has been noted by many philosophers that the results of this study can be explained in alternative ways that do not suggest in favour of the common aspect claim.

Knobe and Prinz predicted that people would be willing to ascribe beliefs, intentions, and other non-phenomenal states to a specific type of non-experiencer - a group agent (Acme corporation)but unwilling to ascribe any phenomenal state to such an agent. This prediction is derived from Knobe and Prinz's hypothesis that people will appeal to their tacit conception of phenomenality when they decide whether an agent can be in the mental state in question. If the given mental state involves phenomenality, then they will deny that the non-experiencer can be in that state; but if not, then they should be more willing to ascribe the state to the non-experiencer (pp. 68-70).

The second study in KP tests the above-mentioned prediction. In this study, they gave people the ten sentences below in random order. The first five sentences involve non-phenomenal states (decide, change, intend, believe, know) while the rest involve so-called phenomenal states (urge, 
joy, imagining, to get depressed and to feel pain). The authors then asked the participants to rate each sentence on a 1-7 scale with 1 being "sounds weird" and 7 being "sounds natural". They predicted that the non-phenomenal sentences would sound more natural than the phenomenal ones, on the plausible assumption that people would consider a company as a non-experiencer. This prediction was borne out, as seen by the means following the sentences (presented in descending order):

Acme Corp. has just decided to adopt a new marketing plan. $(M=6.60)$

Acme Corp. wants to change its corporate image. $(M=6.60)$

Acme Corp. intends to release a new product this January. $(M=6.30)$

Acme Corp. believes that its profit margin will soon increase. $(M=6.10)$

Acme Corp. knows that it can never compete with GenCorp in the pharmaceuticals market. $(M=5.20)$

Acme Corp. is experiencing a sudden urge to pursue internet advertising. $(M=4.70)$

Acme Corp. is now experiencing great joy. $(M=3.70)$

Acme Corp. is now vividly imagining a purple square. $(M=2.70)$

Acme Corp. is getting depressed. $(M=2.50)$

Acme Corp. is feeling excruciating pain. $(M=2.10)$

The authors interpret these results as showing that people distinguish some states from others in the same way philosophers distinguish phenomenal states from non-phenomenal ones. They then argue that the results indicate that people share philosophers' conception of phenomenality, according to which some states but not others have a common aspect- that there is something that it is like to be in those states for the agent. 
Indeed, these results are at first glance in coherence with the common aspect claim. After all, all of the non-phenomenal sentences sounded natural to the participants on average, while most of the phenomenal ones sounded weird on average. And, as Knobe and Prinz note, even the highest mean of the phenomenal sentences (urge) is still smaller than the lowest mean of the non-phenomenal sentences (know). That is, the participants arguably classified the given mental states into two different groups each of which includes either only phenomenal states or only non-phenomenal states. The authors then conclude that this classification is due to people's tacit conception of phenomenality. This conclusion is a hasty one, however, because why the participants treat (most of) the phenomenal sentences as weird while treating the non-phenomenal sentences as natural can quite plausibly be explained without assuming that the folk have a conception of phenomenality.

First, Arico (2010) points out the possibility that the classification of states into two distinct sets might be due to an important difference between the phenomenal sentences and the nonphenomenal sentences rather than the supposed phenomenal difference. He argues that the nonphenomenal sentences provide contextual information about the agent while no such information is given in the phenomenal sentences and that this might explain the difference in responses. To illustrate, while "Acme Corp. believes that its profit margin will soon increase" or "Acme Corp. has just decided to adopt a new marketing plan" include some contextual information explaining what the agent believes or decided, "Acme Corp. is getting depressed" or "Acme Corp. is feeling excruciating pain" does not, simply asserting that the agent is in a particular state without giving any information about the why the agent is depressed or feels pain.

Arico tested this objection by running a variation on the fourth study in KP. That study in KP used two-sentence pairs: "Acme Corp. is feeling upset" / "Acme Corp. is upset about the court's recent ruling", and "Acme Corp. is feeling regret" / "Acme Corp. regrets its recent decision". Knobe and Prinz marked the first sentences in the pairs as phenomenal and the second ones as nonphenomenal. They again asked the participants to rate whether these sentences sound weird or natural. Knobe and Prinz found that the second sentences in these pairs sounded natural to the participants while the first ones sounded weird. Based on this they concluded that people find it problematic to ascribe feeling to a non-experiencer agent (Acme Corp.). Note that unlike the second sentences in the pairs, the first sentences lack any contextual information about what Acme Corp. is feeling regret about or is feeling upset about. 
In his follow-up study, Arico gave participants similar sentence pairs but this time provided equal contextual information. For example, he marked "The Housing Authority is feeling angry about the foreclosures" as phenomenal while treating "The Housing Authority is angry about the foreclosures" as non-phenomenal. He asked participants to rate the sentences in the same way as in KP. He found no significant difference between the sentence pairs. That is, when all the sentences are presented with comparable context, people treated them as equally natural.

Second, Phelan, Arico, and Nichols (2013) point out that the conclusion of Knobe and Prinz draw is problematic because it relies on a tacit and controversial assumption about how people read the group agent term ("Acme Corp."). Knobe and Prinz assume that participants attributed mental states to the group agent itself rather than the members comprising the group. That is, they assume that people read "Acme Corp" in a realist fashion (as an agent over and above the members comprising it). This assumption leads the authors to conclude that participants' denial of the ascription of phenomenal states to the group agent coheres with the common aspect claim. Phelan and colleagues questioned this assumption and tested the possibility that ordinary people interpret group agents in a distributivist fashion (i.e. in terms of its comprising members rather than an agent over and above its members) with two studies. The results of both studies suggest that people treated the group agent in a distributivist fashion. ${ }^{29}$

\footnotetext{
${ }^{29}$ In their first study, Phelan and his colleagues used three different sets of sentences. Sentences of these three sets involved non-mental states, non-phenomenal mental states, and phenomenal mental states, respectively. For example, the first (non-mental) set involved the sentence "After Boeing lost the Army contract, Boeing needed to lay off workers". The second (non-phenomenal) set involved "After Boeing lost the Army contract, Boeing expected to have to lay off workers" and the third (phenomenal) set involved "After Boeing lost the Army contract, Boeing felt anxious about having to lay off workers". They asked the participants to replace the word "Boeing" with either "it" or "they". They plausibly assumed that "they" would indicate the distributivist reading of "Boeing" and "it" would signal the realist reading. Approximately $36.0 \%$ of participants chose "they" to replace "Boeing" in the non-mental sentences while about $61.0 \%$ and $69.0 \%$ of participants chose "they" for the non-phenomenal and phenomenal sentences, showing that the majority of people read group terms in a distributivist way when the term is related to a mental state, whether the state is phenomenal or not. In the second study, Phelan and his colleagues used the same sets of sentences. However, this time they also gave the participants two alternative sentences of the original sentence (selected from one of three sets). The first alternative provided the distributivist reading while the second gave a realist reading. For example, the following alternatives for the phenomenal sentence "the Secret Task Force felt threatened by the public reaction" were given: "The majority of the relevant task force participants felt threatened by the public reaction" and "The task force itself, not just the participants, felt threatened by the public reaction". The participants were asked to select the alternative sentence that they think reflects the meaning of the original sentence better than the other. The results again showed that people chose the realist alternative for non-mental sentences (about 82.0\%), and the distributivist alternative for non-phenomenal sentences (around 58\%) and for phenomenal sentences (around 67.0\%).
} 
These findings raise doubts about KP's conclusion. If the majority of participants in the second study in KP understood "Acme Corp." in the sense of its members rather than the corporation itself, the conclusion that they denied attributing phenomenal states to a non-experiencer would not hold simply because members of the corporations, as humans, are experiencers. In other words, if the participants' unwillingness to ascribe supposed phenomenal states to Acme Corp. is because of the phenomenality these states involve, this means that they thought that members of Acme Corp. are not phenomenally conscious creatures, considering that ordinary people read group terms in the distributivist sense. However, this contradicts the standard understanding of phenomenal consciousness according to which humans (and probably other animals with similar, complex nervous systems) have a phenomenal life. So, the participants' denial appears to have nothing to do with the phenomenality of the mental states.

This reasoning raises an immediate question, however: if people understand the members of Acme Corp. when they read "Acme Corp.", why do they tend to ascribe non-phenomenal states (such as "believing" or "intending") but not phenomenal states (such as "feeling") to Acme Corp.? After all, it is quite plausible to assume that the participants would believe that the members of Acme Corp., as humans, can both believe and feel, for example. Phelan and his colleagues offer an explanation: People do not read the group terms as referring to the members of a group simpliciter. Instead, "group mental state ascriptions are understood in terms of attributions of mental states to group members qua members of the relevant groups" (p. 716). In other words, when people assess whether a state is attributable to a group, they read the group terms as referring to members of the group but also consider whether the members of the given group would have that state or not. For example, it seems more appropriate to say that "Al Qaeda hates America" than to say that "Microsoft hates America" because hating America is something we expect from the members of Al Qaeda while this is not true for the employees of Microsoft (p. 717). If this explanation is accurate, then the participants in KP's study may have thought that it is inappropriate to say members of a company can feel or experience anything about the business of the company whereas they can believe, know, intend, desire or want something about a business topic.

Phelan and colleagues' studies suggest that it is not the best methodological choice to use group agents in experiments to investigate the mental state ascriptions of ordinary people. And this is not the only methodological problem with KP: it also seems to assume that reactions of people to some 
linguistic expressions about mental states straightforwardly reveal their intuitions about those states. Although I believe that investigating how people use a concept or how they react to some sentences that involve the target concept provides important insights into lay intuitions about the target concept, I also believe that we should generally expect there to be a gap between whether one finds a sentence that refers to a mental state to sound weird or natural and her intuitions about the state. One may refrain from using or may find a linguistic expression referring to a state to sound weird for a given agent because almost no one uses that expression for the agent in the linguistic community of the language user, rather than because one thinks that the agent cannot be in the indicated state.

Even setting these worries aside, why participants found phenomenal sentences weird while finding non-phenomenal sentences natural in KP can be explained in alternative ways that have no implication whatsoever for the common aspect claim. To list some alternative explanations, Sytsma and Machery (2009) noted the possibility that participants were unwilling to ascribe socalled phenomenal states to a group agent because of the differences in the behavioural and functional organization between a group agent and individuals. For example, the participants may have thought that Acme Corp. cannot feel anything because it does not have a body (p. 304).

And related worries can be raised. For example, participants may have denied ascribing states like feeling pain or joy to Acme Corp. because they think that corporations lack a self or a soul or life (or some combinations of these) which they may think is needed to feel pain, get depressed and so on. One can object that these possibilities may overlap with and thus do not exclude a lay conception of phenomenality. For example, one can think that Acme Corp. cannot feel anything because for an agent to have phenomenal experiences, one needs to be alive. However, there seems no reason to assume that the lay concept of life involves a conception of phenomenality. That is, laypeople do not need to recognize any common, qualitative aspect between perceptual cases and bodily sensations to believe that only living organisms can feel. One who believes that only living organisms can feel can also consistently believe, for example, that non-living entities can see colors or smell roses. Similarly, it is perfectly possible for one who thinks that only entities with a soul can feel pain, and thus that robots (who do not have souls) cannot feel pain, to nonetheless hold that robots can see colors. Further, assuming that the lay concepts of soul or life or self 
involves the concept of phenomenal consciousness begs the question of whether laypeople have such a concept.

To summarize, the second study in KP does not present compelling evidence for the common aspect claim. This is because the study has some serious methodological problems, including asking participants to rate the naturalness of sentences, their choice of non-experiencer agent, and the difference in the contextual information given in the phenomenal and non-phenomenal sentences. And even if these problems, taken together, had a negligible effect, the results of the study can be read in other plausible ways that have nothing to do with phenomenal consciousness. Of course, it does not follow that the common aspect claim is false from the fact that the study discussed fails to provide compelling support for the claim. For this, we would need positive evidence suggesting that the common aspect claim is false.

\subsubsection{Sytsma \& Machery’s Study}

The most prominent empirical study that suggests against the common aspect claim comes from SM. Like the second study in KP, the first study in SM directly targets the question of whether laypeople have a sort of conception of phenomenality; but their study does not have the methodological problems that we saw plague KP. In particular, SM does not involve a group agent or involve an assessment of the naturalness of sentences. Instead, their study compares participants' willingness to attribute two prototypical examples of so-called phenomenal states (seeing red and feeling pain) to a human being and a simple robot.

The research question behind SM was what characterizes the lay conception of subjective experience. As they point out, most contemporary philosophers believe that "subjective experience is characterized by its phenomenality" (p. 300). Sytsma and Machery designed their study to compare folk and philosophical conceptions of subjective experiences. The rationale behind the study was straightforward: If the folk and philosophical conceptions of subjective experience coincide, then we should expect laypeople to tend to treat the paradigm cases of phenomenal states in a similar way to how (most) philosophers do. More specifically, since humans, but not simple robots, would be regarded as experiencers, we should expect both philosophers and laypeople to 
happily attribute seeing red and feeling pain to human beings but deny ascribing both states to a simple robot.

The study was conducted on both philosophers and laypeople. Participants were randomly given one of four scenarios that varied two factors - the agent and the mental state. In half of the scenarios, the agent was a normal human named "Timmy", whereas the other half involved a relatively simple robot named "Jimmy" who has a camera, wheels and two grasping arms with touch sensors. The scenarios involving Timmy or Jimmy were further divided into two versions. The first version involved "seeing red" while the second involved "feeling pain". In the first version, Timmy/Jimmy is instructed to move a red box from a set of boxes with different colors. Timmy/Jimmy moves the red box. Participants were asked if Timmy/Jimmy "saw red". The second version starts in the same way as the first version: Timmy/Jimmy is instructed to move the red box. However, this time Timmy/Jimmy is given an electrical shock after he/it picks up the red box. The agent drops the box and moves away from it. Participants were then asked whether the agent "felt pain".

The results showed that, as expected, both non-philosophers and philosophers were keen to ascribe seeing red and feeling pain to Timmy. Both groups were also similar in denying that Jimmy felt pain. However, they significantly differed in their willingness to attribute seeing red to Jimmy: Philosophers, as expected, tended to deny that Jimmy saw red while non-philosophers tended to ascribe seeing red to Jimmy. ${ }^{30}$

Sytsma and Machery plausibly conclude from these results that "in clear contrast to philosophers, the folk do not seem to believe that there is something common to all these mental states - namely that they are phenomenal" (p. 319). They then take this to suggest that laypeople lack the concept of phenomenal consciousness. Put in the terms introduced above, these results suggest that laypeople by and large do not share common aspect intuitions. After all, if they recognized the supposed common phenomenal aspect between seeing red and feeling pain, they would have the intuition that a simple robot can neither see red nor feel pain. However, this is not how they by and

\footnotetext{
${ }^{30}$ On the 1-7 scale, the mean for Jimmy's seeing red was 5.15 for non-philosophers and 3.48 for philosophers, and for Jimmy's feeling pain the corresponding means were 2.54 (non-philosophers) and 2.02 (philosophers).
} 
large responded, suggesting that laypeople do not tend to treat these states as phenomenal states. Considering that both states are paradigmatic examples of phenomenal states, however, it is plausible to think that they do not have a tacit conception of phenomenality.

The empirical findings in SM raise an important challenge for the common aspect claim. However, the second study in Peressini (2014) presents empirical findings that potentially run against the conclusion of SM and support the common aspect claim. The next section will discuss whether these findings support the common aspect claim or not. Further, Sytsma and Machery's interpretation of the asymmetry between lay attributions of seeing red and feeling pain is not free from objections. 4.3 will discuss these objections, focusing on the most common and plausible objection - the Ambiguity Objection. The next chapter will then introduce new experiments in reply to the Ambiguity Objection.

\subsubsection{Peressini's Study}

Peressini (2014) includes two parts. The first part investigates whether laypeople treat robots and other artefacts as experiencers or not. This part of the study will be discussed in connection with the Ambiguity Objection in $\$ 4.3 .2$. The second part directly investigates whether people have a conception of phenomenality. This section will discuss whether this second part of Peressini's study provides compelling evidence for the common aspect claim.

Based on the results of the second part of his study, Peressini claims that laypeople have a concept of phenomenality. This is based on the mean responses to two critical questions-questions 3 and 6 (p. 883). ${ }^{31}$ Question 3 reads: "Can we ever be sure that you see red the way another person does?" The mean response was below and significantly different from the neutral point, indicating general disagreement. Peressini then interprets this result as follows:

Question 3 shows that the participants' understanding of 'seeing red' involves a component that cannot be verified from a third-person perspective. Such a private and ineffable

\footnotetext{
${ }^{31}$ Peressini also claims that the folk conception of phenomenality differs notably from the philosophical conception in that the folk conception does not lead to the hard problem based on the responses to two other questions (Question 1 and 8). For present purposes, however, I will focus on Questions 3 and 6, leaving the question of what the responses to Questions 1 and 8 indicate to $\S 7.1$, where I evaluate the prevalence of conceivability intuitions.
} 
component is typically a defining feature of qualia or "phenomenality." The point, however, is that there must be "something it is like" to see red for it to be unverifiable. (p. 883)

As for Question 6, participants were first given the following prompt: "Imagine that a completely color-blind person got an implant that encoded colors in her visual field with numbers indicating colors, so for example, the sky on a clear day was indicated with a number 1 to indicate blue". Then they were asked, "Would such a person be able to see blue?" The mean response was again below and significantly different from the neutral point. Peressini interpreted this result as showing that for laypeople, “"seeing blue' involves a 'qualitative aspect' or at least a constraint on 'what it would be like' for a phenomenally conscious entity for it to 'see blue' as opposed to detecting blue" (p. 884).

Based on the low mean responses, Peressini claims that "something like phenomenality is required to explain the subjective and qualitative components brought out in questions 3 and 6" (p. 883). Below, I will discuss whether the results truly support this claim.

An immediate worry about Peressini's interpretation of the answers to Question 3 is noted by Sytsma (2018): the respondents may have thought that it is impossible to verify two people see red in the same way for practical reasons. After all, we are not typically able to be sure about whether two people see the same thing in the same way. So, the low mean response to this question does not mean that people think that such verification is impossible in principle. A more warranted way to understand whether people treat "seeing" red as involving a component that cannot be verified from a third-person perspective, Sytsma suggests, would be asking people about whether it was theoretically possible that scientists in the future might be able to verify that two people see red in the same way.

The low mean of responses to Question 3 nonetheless may be considered as suggesting that the lay conception of seeing colors involves the aspects of ineffability and private access. These aspects, as Peressini points out, are usually ascribed to phenomenal states. However, it is a further claim that these features are sufficient for a mental state to be phenomenal. It is one thing to ascribe these features to a mental state and another thing to ascribe phenomenality to that state. For example, I believe that no number of words is enough to describe what a particular color looks like 
(without invoking other colors). Verbal descriptions can only trigger one's imagining a color but cannot give information about what a particular color looks like. So, you cannot describe the blueness of clear sky to a person born blind because she cannot imagine any color. However, there is no need to think that this is because of the phenomenal aspects of colors. Perhaps the color information is not encoded in the brain in the same way propositional or linguistic information is encoded. ${ }^{32}$ So, linguistic expressions may lead us to imagine colors, but they cannot create or convey color information. So, it is no wonder that color sensations are ineffable and private. However, as a phenomenal anti-realist, I also believe that it is possible for a super-scientist with sufficient technology in the future to access what colors look like to one from a third-person perspective. In brief, one's ascription of ineffability and privacy to color sensations does not mean that one finds phenomenality in them, and thus there is no reason to think that the low ratings for Question 3 necessarily support the common aspect claim.

Unlike ineffability and private access, the qualitative aspect is usually regarded as the core of phenomenal states and is arguably both necessary and sufficient for being phenomenal. Even the most liberal sense of phenomenality or qualia involves a reference to this aspect although what it exactly means is far from being certain. As a result, it is common for phenomenal anti-realists to dismiss this aspect altogether. So, if people found a qualitative aspect in seeing colors, this would support the idea that people have a certain sort of conception of phenomenality. Peressini explains participants' resistance to attributing "seeing blue" in their reply to Question 6 (would a completely color-blind person who got an implant that encoded colors in her visual field with numbers indicating colors be able to see blue when she looks up the clear sky?) with their recognition of a qualitative aspect in seeing blue: folk attributions of seeing colors to an experiencer involves a qualitative aspect (blueness). However, it is a big leap from the low mean of the responses to Question 6 to the claim that people find a qualitative aspect in seeing blue. The participants may simply have thought that seeing blue requires seeing a colored object in the first place (rather than seeing numbers indicating colors) and the person, in the case described, does not see any colored object. So, it is very natural for them to deny that the given case involves seeing blue. Despite

\footnotetext{
${ }^{32}$ See Churchland (1989) for an account of how the knowledge of what it is like to see red differs from the propositional knowledge about redness. Similarly, Musacchio (2002) defends the view that not only visual but also other types of sensory knowledge differ from propositional knowledge in this way.
} 
being a phenomenal anti-realist, I would also give the lowest rating for this question. In brief, the participants' denial of seeing blue can be explained easily without appealing to the qualitative aspect of blueness.

To sum, since we can explain the results that Peressini takes to be suggestive of a lay conception of phenomenality in alternative and plausible ways that have nothing to do with phenomenal consciousness, pace Peressini, the low responses to these questions do not cast considerable doubt on SM's conclusion. For the same reason, the results for these questions do not provide us with convincing evidence for the common aspect claim. As noted above, however, SM's conclusion against the common aspect claim has not been free from direct objections. The next section will discuss these objections focusing on the Ambiguity Objection.

\subsection{Objections to SM}

Remember that in SM, the non-philosopher respondents by and large differed from the philosophers in their willingness to ascribe seeing red to a relatively simple robot (Jimmy) while both groups tended to endorse that the robot cannot feel pain. Based on this, Sytsma and Machery concluded that laypeople do not seem to find a common, phenomenal aspect in seeing colors and feeling pain. And considering that seeing colors and feeling pain are prototypical examples of phenomenal states in the literature, this suggests that laypeople lack the concept of phenomenal consciousness.

This conclusion can be formulated in an argumentative way as follows:

(1) If laypeople have the philosophical conception of phenomenality, then they should treat prototypical examples of phenomenal states similarly, either denying both of the robot or ascribing both to the robot.

(2) Laypeople treat seeing red and feeling pain dissimilarly, suggesting that they do not see the common, "phenomenal" aspect in seeing red and feeling pain.

$\therefore$ Therefore, laypeople by and large do not share the philosophical conception of phenomenality because they do not recognize a common "phenomenal" aspect in the paradigm cases of phenomenal consciousness. 
If the conclusion is true, it is natural to think that laypeople do not have any sort of conception of phenomenality, and relatedly they do not share common aspect intuitions. However, one could accept the conclusion and yet hold that while people do not share the philosophical conception, they might have a conception that nonetheless deserves to be treated as a conception of phenomenality. The objector might note that according to the philosophical conception of phenomenal consciousness, seeing red is a phenomenal state. Also, in this conception, (at least) simple robots are not phenomenally conscious entities, which is why philosophers in SM denied that Jimmy could see red. But perhaps, the objection goes on, laypeople think that seeing red, unlike feeling pain, is not a phenomenal state, which would explain why the participants in SM tended to accept that a simple robot can see red. Or, perhaps they think that robots, like humans, are phenomenally conscious entities so they can see red, but they cannot feel pain because of other reasons, most plausibly because they lack the appropriate bodily organization.

The problem with this objection is that it seems ad hoc. This is because if the lay conception of seeing red and feeling pain is so different from the philosophical conception, what reasons do we have to assume that this lay conception may still involve a conception of phenomenality? If people do not recognize the supposed phenomenality involved in seeing colors, it is quite reasonable to assume that they do not recognize phenomenality in other cases including feeling pain, considering that colors are indispensable in the "phenomenal" jargon. It would be far-fetched to claim that laypeople treat feeling pain as a phenomenal state although they do not regard seeing color as such. Such a claim would require us to believe that laypeople see no qualitative aspect in colors while they acknowledge the qualitative nature of painful experiences. But which reason do we have to believe that painful experiences, but not colorful ones involve the supposed qualitative aspect for laypeople? So, the objector owes us an explanation of the discrepancy between seeing colors and other so-called phenomenal states in the alleged lay conception of phenomenality because there seems no reason to believe that there might be a folk conception of phenomenality that excludes one of the most typical examples (if not the most typical one) of the philosophical conception of phenomenal consciousness. The same discrepancy poses a problem also for the possibility that people treat robots as experiencers. If people think that robots' computational processes suffice to enjoy phenomenal states and thus they can see colors, why then do not they also think that it does not suffice to feel pain? If it is claimed that they deny that robots can feel pain because they lack 
the appropriate bodily organization to feel pain, this would suggest that the folk conception of pain consider pain as a bodily state rather than a mental state.

Against this reasoning, one might claim that people tended to judge that a simple robot can see colors while rejecting that it can feel pain because feeling pain has a valence, or hedonic value, whereas seeing colors does not. After all, this explanation is the one defended by Sytsma and Machery in their original paper. Expanding on this, one might argue that perhaps laypeople tend to have a conception of phenomenal consciousness that only includes subjective experiences with a hedonic value. This conception, the objector goes on, while different from the philosophical conception, should still be considered as a conception of phenomenality because it is natural to think that laypeople would further endorse that there is something that it is like to have these experiences.

It should be admitted that this is possible. However, we have empirical reason to think that SM's Valence Hypothesis is not true, based on a further series of studies in Sytsma (2012). In his first study, participants were asked whether the simple robot used by SM can smell caramel (positivevalence), rotting meat (negative-valence), or newspaper (valence-neutral). Sytsma found no significant difference in participants' answers for these three items, despite the fact that they vary considerably in valence. The second study in Sytsma (2012) also suggests against the Valence Hypothesis. In this study, participants' answers for caramel (positive-valence), paint thinner (negative-valence), and dry dirt (valence-neutral) did not significantly differ.

One might also point out the possibility that the participants in SM's study tended to endorse that Jimmy can see colors while rejecting that it can feel pain not because they don't have a conception of phenomenality, but because the simple robot does not satisfy the enabling conditions that need to be in place in order for the robot to feel pain, (e.g., being made of flesh, or having at least certain connections between the site of damage and the robots CPU). ${ }^{33}$ That is, it is possible that their conception of phenomenality is such that it does not categorically reject that simple robots phenomenally conscious entities and allows that they can have the relevant states so long as their

\footnotetext{
${ }^{33}$ Thanks to Kevin Reuter for bringing up this possibility and for the objection based on the Valence Hypothesis.
} 
bodily organisation satisfy the enabling conditions. The objector might then contend that the participants in SM's studies might have tended to judge that Jimmy satisfies the enabling conditions for seeing colors while failing to satisfy the conditions for feeling pain.

This is also possible. However, I argue that this does not undermine SM's conclusion. The reason is that to be considered as enabling conditions for an entity to have a phenomenal state, in the relevant sense for this thesis, those conditions should have to do with the entities mind. This is because, pain is a mental quality according to phenomenal realism, at least in its standard version as used in this thesis. And we have empirical evidence to think that for laypeople the enabling conditions that need to be in place in order for the robot to feel pain are bodily conditions rather than mental conditions. Thus, Study 3 and Study 5 in Sytsma (2012) suggest that laypeople tend to think that the enabling conditions for a robot to be able to feel pain are bodily conditions rather than mental conditions.

In Study 3, two vignettes described "an adult human (Susan) who lost her right hand in an accident and had it replaced with either a robotic hand (hard and metallic) or a bioengineered hand (soft and fleshy)" (Sytsma, 2012, p. 190). In both cases, Susan was described as capable of doing all of the things with her new hand (hard and metallic, or soft and fleshy bioengineered hand) that she did with her old hand. After reading these vignettes (one with robotic hand condition and other with bioengineered hand condition), the participants were asked whether "Susan felt pain when she picked up the hot skillet" (p. 191). The participants were, by and large, keen to accept that Susan felt pain when she had the bioengineered hand, while rejecting that she felt pain when she had the robotic hand. But note that the mind of Susan was constant in the vignettes; the only difference was a bodily difference, namely the hand of Susan. Study 5 was very similar to Study 3, except this time the agent was Jimmy (with the same robotic or bioengineered hand). The answers were also similar. The majority of the participants endorsed that Jimmy felt pain when it was equipped with a bioengineered, while rejecting that Jimmy felt pain when equipped with a robotic hand. And the difference between these two conditions was significant.

Based on these considerations above, attacking Premise 1 or 2 seems more plausible than claiming that SM's conclusion coheres with a possible lay conception of phenomenality that significantly differs from the philosophical conception. I cannot imagine any objection to Premise 1 especially 
considering that the philosophers in the study treated seeing red and feeling pain similarly. However, there is a common way of attacking Premise 2: Claiming that "seeing red" is ambiguous in Premise 2. That is, the majority of the participants in SM's study might be happy with attributing seeing red to a robot in the sense of identifying or detecting the red objects by making calculations on the relevant visual information (which we may call the informational sense) rather than in the sense of seeing qualitative property of redness (which can be called as the phenomenal sense). This objection, which Sytsma (2014a) calls the "Ambiguity Objection", is the most popular objection to SM and will be discussed in the rest of this chapter.

According to the Ambiguity Objection, philosophers and non-philosophers read "seeing red" in different ways in SM: While philosophers tend to understand "seeing red" in the phenomenal sense, laypeople tend to take it in the informational sense. This is then taken to explain why the majority of non-philosophers were happy with ascribing seeing red to a simple robot, while the majority of philosophers were not. The basic idea, here, is that the participants in SM thought that robots can see colors in the informational sense rather than in the phenomenal sense. And if this is the case, the dissimilarity in lay ascriptions of seeing red and feeling pain to a robot does not suggest that they do not recognize phenomenality in these states, which falsifies Premise 2. This objection has been defended by Huebner (2010); Fiala, Arico, and Nichols (2014); Peressini (2014) and recently by Chalmers (2018).

In their original paper, Sytsma and Machery offered two replies to the Ambiguity Objection. They first noted that the objector owes us an explanation of why the participants differed from the philosophers in reading "seeing red" in the informational sense if the objection is to avoid being ad hoc (p. 310). After all, if the objector were right, we would expect that a considerable proportion of the participants would answer negatively with the rest answering positively. However, the great majority of the respondents (around 85.0\%) gave answers that are equal or superior to the neutral point. So, the objector should explain why the overwhelming majority of the participants chose the informational sense of "seeing red" over its phenomenal sense. Second, they noted that while the philosophers who denied that Jimmy can see red tended to explain their answers in terms of phenomenal consciousness, the explanations given by non-philosophers who denied this do not indicate a phenomenal reading; rather, if anything, these explanations signal that they construe seeing as an ability that only humans and animals possess. 
Sytsma (2009) offers a further response: the Ambiguity Objection reflects a theoretical view of colors that makes a distinction between physical red (surface reflectance and other optical factors, and so on) and phenomenal red (redness as experienced by us) (p. 966). He argues, however, that the folk conception of colors seems to be a naïve realist view that does not make such a distinction. Indeed, the studies discussed in $§ 4.1 .1$ suggest that the folk conception of colors (and pains) coheres with naïve realism. Recall that the majority of participants endorsed that colors are located in the perceived object rather than in the mind and that colors would still be there even if there were no perceiver. So, it seems far-fetched to expect that the participants in SM made a distinction between physical and phenomenal red and ascribed "seeing red" for the simple robot in the physical sense of "red".

As noted, the Ambiguity Objection claims that the participants who gave a positive answer to the question of "Did Jimmy see red?" thought that Jimmy could identify or detect red rather than see red in the phenomenal sense. So, the objector would predict that if participants were given the options "Jimmy detected red" or "Jimmy identified red" in addition to "Jimmy saw red", they would be much less keen to ascribe seeing red to Jimmy. Fiala et al. (2014) conducted an empirical study to evaluate this prediction, which will be discussed in the next sub-section. Another empirical study related to the Ambiguity Objection is the first part of the study presented in Peressini (2014). As will be seen, Peressini presses the Ambiguity Objection against SM's conclusion by claiming that laypeople make a categorical distinction between experiencers and non-experiencers, classify robots as non-experiencers, and tend to treat experiential expressions (such as "see red") in the informational sense with regard to non-experiencers. He takes the first part of his study to support these claims, as I will discuss in $§ 4.3 .2$. I will argue that the results do not support Peressini's version of the Ambiguity Objection, however. Further, Peressini is silent on a general, pressing issue for the Ambiguity Objection: Why do people tend to read "seeing red" in the informational sense while reading "feeling pain" in the phenomenal sense? Chalmers (2018) offers a potential response to this question, however, which will be discussed in $\$ 4.3 .3$.

\subsubsection{Testing a Prediction of the Ambiguity Objection}

Fiala et al. (2014) used roughly the same vignette as SM with the exception that the color of the target box was green instead of red and the robot vignette removed the anthropomorphic 
language.$^{34}$ For each vignette, they asked participants to select each statement they thought was true from a list of five statements in the following fixed order:

Timmy/Jimmy detected green.

Timmy/Jimmy saw green.

Timmy/Jimmy located the green box.

Timmy/Jimmy identified the green box.

Timmy/Jimmy moved the red box.

The last statement was used as an attention check and the participants who selected this statement were excluded from the sample. Fiala, Arico, and Nichols (hereafter, FAN) found that only $28.0 \%$ of the participants selected the statement "Jimmy saw green" while $60.0 \%$ selected "Jimmy detected green". The corresponding figures for Timmy were $57.0 \%$ and $50.0 \%$. Based on these results, FAN concluded that the participants in SM agreed with the statement that Jimmy saw red just because they wanted to convey their belief that Jimmy detected and identified the red box, not because they thought that Jimmy saw red in the same way that we see colors.

In reply, Sytsma (2014) noted that there might be something that went awry in this study since only $57.0 \%$ of the participants answered that the normal human, Timmy, saw green and only half the participants answered that he detected green, despite the fact that both of these would seem to clearly apply. Sytsma lists some potential issues that may have caused these surprising results: (1) the sample size was small and not restricted to non-philosophers; (2) the first two statements involved references to just the color (detecting and seeing green), while the third and the fourth statements involved references to the colored object (located and identified the green box); (3) there might be an order effect in favour of the first statement over the second; and, (4) people

\footnotetext{
${ }^{34}$ In their non-anthropomorphic version, Jimmy was always referred to as "Jimmy" instead of "he".
} 
might think that at least one of the options must be wrong and thus be reluctant to confirm "Jimmy saw red" in addition to endorsing other options.

To control for these potential issues, Sytsma conducted a series of four studies, which were designed to sequentially address these problems. The vignette for each was the same as the probe of FAN except the target color was blue instead of green. Study 1 otherwise replicated FAN's study, but with a larger sample size and restricting the participants to non-philosophers. Study 2 changed the first two statements to "Timmy/Jimmy detected the green box" and "Timmy/Jimmy saw the green box". Study 3 was the same as Study 2 except it reordered the answer choices so that "saw green" was followed by "located", "identified" and "detected" respectively. And, finally, Study 4 removed the option involving “detected green”. Figure 1 compares the results of FAN's study to the results of Sytsma's four studies in terms of the percentages of the respondents who selected that Jimmy detected the color and the percentage who selected that Jimmy saw the color.

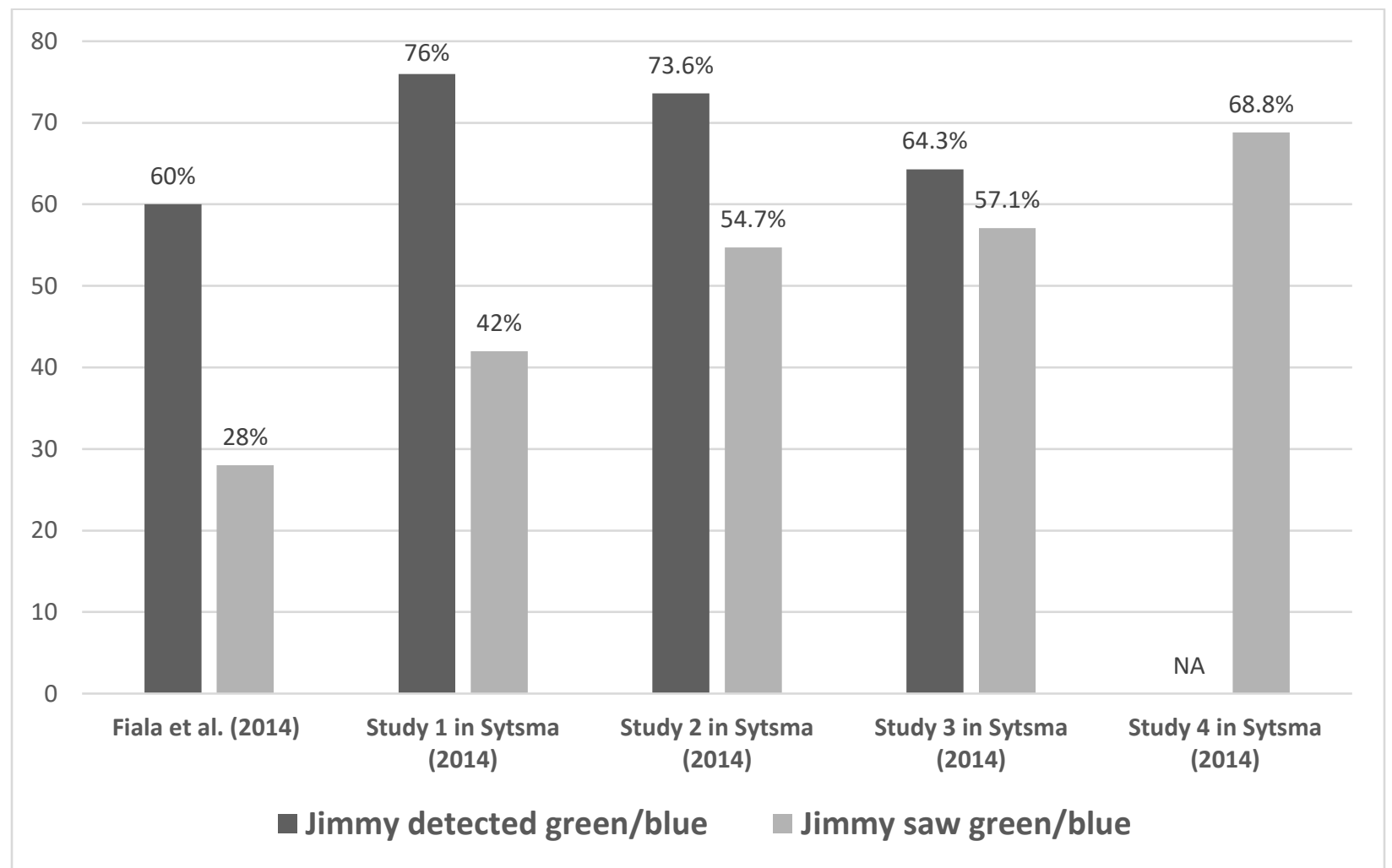

Figure 1: Comparison between the results of Fiala et al. (2014) and four studies of Sytsma (2014). 
In Study 1, the proportion of respondents endorsing that Jimmy saw green significantly increased from $28.0 \%$ in FAN's study to $42.0 \%$. This increased further in each subsequent study: $54.7 \%$ in Study 2, 57.1\% in Study 3 and 68.8\% in Study 4. Sytsma concludes that these results undermine FAN's objection to SM because the majority of the participants endorsed that a simple robot could see colors even if they were given some more informational alternatives (detect/locate/identify green/green box). These findings also suggest against the Ambiguity Objection in general.

Sytsma used "Timmy/Jimmy detected/saw the blue box" instead of "Timmy/Jimmy detected/saw blue" in his studies 2-4 because of potential issue (2). However, it might now be objected that the statement "Timmy/Jimmy saw the blue object" is problematic in that "saw the blue object" is more likely to be read in the informational sense compared to "saw blue". To put the point differently, the objector might claim that (2) is not a valid issue because worrying about the difference between seeing a color and seeing a colored object is to miss the main point of the Ambiguity Objection: detecting/identifying/locating a color roughly means detecting/identifying/locating the colored object whereas seeing blue does not mean seeing the blue object. This is because, the objection goes, seeing colors in the phenomenal sense requires one to see/experience a qualitative, experiential property (greenness, blueness, and so on) rather than tracking the colored object, which Sytsma (2009) already noted as discussed in $\$ 4.3$ in relation to Ambiguity Objection. So, when the participants read the statement that "Jimmy saw the blue object", some of them might treat the statement in the informational sense (in the sense of detecting/identifying/locating the blue object) rather than in the phenomenal sense.

This point might in part explain the relatively small differences between detecting blue and seeing blue in Studies 2 and 3 (around 19.0\% in Study 2 and around 7.0\% in Study 3) in comparison to the difference in Study 1 (34.0\%) and the study by FAN (32.0\%). However, the point alone cannot explain the increased proportion of those endorsing that Jimmy saw blue in Sytsma's study because there was no difference in the statements across Studies 2-4, and yet the percentage selecting the "saw" statement increased across these studies. This suggests that issues (1), (3) and (4) remain, and thus we should not dismiss the high proportion found in Study 4 just because the statement that "Jimmy saw the blue box" might still be somewhat ambiguous. 
Against this reasoning, one might argue that Study 3 and Study 4 should not be considered as improvements on the potential issues (3) and (4) because Study 3 just reordered the statements, and Study 4 did not give the participants the chance to compare "seeing blue" with "detecting blue". Although this is true, we cannot dismiss the key result of Study 3 (57.1\% endorsed that Jimmy saw blue) because of the alternative order (saw/detected/locate/identified) in the study, just as we cannot dismiss the results of FAN's study because of its order. As for the objection against Study 4 , it should be noted that although the study removed "detecting blue", the participants still had the chance to compare "seeing blue" with "locating blue" and "identifying blue". So, again, we cannot dismiss the results of Study 4. As a matter of fact, we have a plausible reason to find the results of the studies in Sytsma (2014) more reliable than FAN's study because in all of these studies the results for Timmy's seeing and/or detecting the target color are higher, and hence more plausible, than in FAN's study. ${ }^{35}$

Further, even if we take the accurate proportion of laypeople endorsing Jimmy's capability of seeing colors as $42.0 \%$, as found in Study 1 (in which the statement was "Jimmy saw blue"), this still poses a problem for the Ambiguity Objection and the common aspect claim: If laypeople recognize two senses of "seeing blue" (informational and phenomenal senses), and if phenomenal consciousness is phenomenologically manifest to laypeople, the overwhelming majority of them should deny "seeing blue" for the robot. After all, the participants had the chance to convey the idea that a robot can detect and identify colors but cannot see the color itself. However, Study 1, which just repeats FAN's study with a larger sample size and more appropriate participants (only non-philosophers), does not confirm this prediction.

\subsubsection{Peressini's Version of the Ambiguity Objection}

As noted, the standard experimental way to investigate the folk conception of subjective experience is to look at the responses of laypeople to the questions about whether different types of agents (humans, robots, animals, etc.) can entertain phenomenal states or not. Peressini (2014)

\footnotetext{
${ }^{35}$ In Study $167.5 \%$ endorsed that Timmy saw the target color. This rose to $80.9 \%$ in Study 2, $76.7 \%$ in Study 3 and, finally, $77.8 \%$ in Study 4. Similarly, in Study $172.5 \%$ endorsed that Timmy detected the target color, followed by $63.8 \%$ in Study 2 and $58.2 \%$ in Study 3 Sytsma (2014, pp. 58-61).
} 
asserts that this standard way presupposes that the participants take all types of agents as experiencers. However, Peressini suspects that people intuitively carve up the world first and foremost in terms of different kinds of entities - experiencers and non-experiencers - rather than different types of mental states. If this is the case, he holds that asking people to rate their attributions of experiential mental states to the entities that they see as non-experiencers would be as meaningless as asking "is it more efficient for a pig to fly or breath underwater?" (Peressini, 2014, p. 863). He charges SM of missing this important point: if people understood robots as nonexperiencers, then they would find the question of "did Jimmy see red?" meaningless and they would then be forced to take "seeing red" in the informational sense rather than the phenomenal sense. Peressini contends that his view explains why people adopt the informational reading of "seeing red": laypeople see robots as non-experiencers and this leads them to read "seeing red" in a non-phenomenal, informational way for the robot in SM while reading the phrase in the phenomenal sense for the corresponding human. His view also attempts to explain why the overwhelming majority of participants preferred an informational reading over a phenomenal reading: If laypeople's tendency to read "seeing red" in the informational sense for a robot is a systematic process, it is no wonder that almost all people take "seeing red" in the informational sense.

In this way, Peressini offers needed context for the application of the Ambiguity Objection, giving an explanation for why the majority of people would tend to read "seeing red" in the informational sense for the robots while adopting a phenomenal reading for humans. The first part of Peressini's study attempts to provide empirical support for this view by testing whether people tend to categorically reject that artefacts are experiencers. In the rest of this section, I will discuss whether Peressini's results in fact support his view.

In the first part of Peressini's study, participants were first given the following definition of “experiencer":

As we all know, each of us as conscious human beings have an "inner life." We are aware of things going on around us and inside our minds. In other words, there is something it is like to be each of us at any given moment: the sum total of what we are sensing, thinking, feeling, etc. We are experiencers. 
On the other hand, things like thermostats, burglar alarms, and bread machines do not have an inner life: there is not anything it is like to be those objects, despite the fact that they can monitor conditions around them and make appropriate things happen at appropriate times. They are not experiencers. (pp. 868-869)

Following this statement, participants were asked to rate 20 entities in terms of their being experiencers. ${ }^{36}$ The normal human beings (your best friend and Helen Keller), the dolphin, God, the person under general anaesthesia, Bambi, the human embryo, Spock (from Star Trek) and the person in coma were clearly treated as experiencers. In contrast, robots and computer systems were treated as non-experiencers. ${ }^{37}$ Considering these results, Peressini reaches the conclusion that "it is hard not to see this as evidence for a deep-seated intuition that artificial entities are categorically not experiencers" (p. 882).

Although the results clearly suggest that the participants, by and large, denied that robots are experiencers, what they understand by "experiencers" and relatedly why they denied that robots are experiencers is far from clear. As Sytsma (2018) points out, the results of Part 1 in Peressini's study pose a threat to SM's conclusion only if the results clearly show that the lay conception of being an experiencer is tied to a conception of qualitative character, which Peressini labels "qcharacter": Blueishness of the experience of seeing clear sky, for example (pp. 868-883). That is, to establish the claim that people are forced to read "seeing red" for robots in the informational sense rather than the phenomenal sense, the results should suggest that people think that robots are not experiencers because they cannot have any experience with qualitative character (they cannot experience blueishness of the clear sky, for example).

One can think that the participants understood from Peressini's prompt that an "experiencer" is an agent that can enjoy experiences with qualitative character. And if so, the results of Peressini's study can be considered as showing that laypeople have a conception of phenomenality given that

\footnotetext{
36 Entities included "your best friend", a number of fictional robots (such as two Star Wars robots), some computer systems (such as and Data from Star Trek) as well as different forms of living organisms and God.

${ }^{37}$ On the 1-7 scale, "your best friend" received the highest mean (6.97), followed by Helen Keller (6.55). Among the computer systems/robots, C3PO and Data (both from star trek) received the two highest mean (3.21 and 3.07, respectively). See Peressini (2014, p. 870) for the means of other entities.
} 
they endorsed that a person is an "experiencer" while denying that robots were "experiencers". 38 Admittedly, this probably was the case for some participants. However, we have good reasons to doubt that the majority of participants gave this pattern of responses because their conception of "experiencer" was tied to qualitative character of experiences.

Firstly, participants' rejection might be due to some concerns motivated by Peressini's prompt, but these concerns might have nothing to do with the qualitative character of experiences. One can, for example, read the expression "inner life" (in the prompt defining "experiencer") in the sense of being alive or having a life. Such a person would think that robots are not living entities, so they cannot be experiencers according to the prompt. Note that such a person might still think that robots nonetheless can see colors in the same sense in which we see colors. The part in the prompt that summarizes why we are experiencers ("there is something it is like to be each of us at any given moment: the sum total of what we are sensing, thinking, feeling, etc.") might also factor in participants' rejection. One may think that the sum total of a robot's capabilities of "sensing, thinking, feeling, etc." is not enough to consider it an experiencer. For example, one may think that robots can sense very well but they are not experiencers because the sum total of their capabilities of sensing, thinking, feeling is not enough to consider them as experiencers (e.g. they do not have or have very limited capability of thinking and feeling). Note again that the same person may still think that robots can see the blueishness of the clear sky.

Second, as Sytsma (2018) points out, the prompt clearly compares humans to artefacts, and because of this participants may have thought that their task was to rate the entities based on their proximity to normal humans or whether they are artefacts or not. And, indeed, the order of the mean values for entities corresponds with the similarity of the entities to a normal human being. So, it is quite possible that the participants thought that robots and other artefacts cannot be experiencers because of their dissimilarity to humans. And this would not clearly have anything to do with the qualitative character of experiences.

\footnotetext{
38 Thanks to Dan Weijers for emphasizing this possibility.
} 
Lastly, Sytsma also notes that the second part of Peressini's study (which I discussed in the previous section with regard to the question of whether laypeople have a conception of phenomenality) seems to suggest that the lay conception of "experiencer" is not tied to the qualitative character (p. 21). For example, when Peressini interprets the high ratings for a question (Question 7-Version A) asking whether a non-human but intelligent creature is an experiencer, he notes that the lay intuition that robots are not experiencers does not seem to be based on qualitative character (p. 882). In line with this, it is also worth noting that, participants in reply to Question 9 tended to treat a person "whose senses were destroyed by disease, but who had been given artificial senses that reported directly to their speech centre things like 'wall up ahead' or 'bird singing ten feet to right' " (p. 875) as an experiencer. This suggests that for participants, having qualitative character is not necessary for an entity to be an experiencer. Based on this, Sytsma asks, "if the concept of experiencer that laypeople are operating with is not tied to q-character, then it is not clear why people classifying robots as non-experiencers would explain their reading 'see red' in the informational sense as opposed to a phenomenal sense that centrally features $q$ characters, as the objection contends" (p. 21).

Because of the considerations above, Peressini's findings do not offer compelling support for his claim that people are forced to adopt the informational sense of "seeing red" with regard to robots. Further, there is a clear problem with Peressini's version of the Ambiguity Objection. Let's assume Peressini's claim is true and the participants in SM's study were led to employ the informational sense of "seeing red" because they have a deep-seated intuition that artefacts are not experiencers. If SM's participants were thinking this way, then they should have also read "feeling pain" in the informational sense and judged that robots can feel pain in the sense of detecting or recognizing that a body part was damaged. However, in SM, the great majority of subjects denied that Jimmy could feel pain. Peressini is silent about this asymmetry. This does not mean that no explanation is available, and the next section will consider one possibility proposed by Chalmers.

\subsubsection{A Possible Explanation of the Difference between Lay Treatment of Seeing Red and Feeling Pain}

As noted above, a common problem with the Ambiguity Objection is that even if it is true that people draw a distinction between seeing colors in the informational sense and in the phenomenal sense, and adopt the informational sense with regard to robots, an important question immediately 
arises: Why do they then treat "feeling pain" in the phenomenal sense and reject robots being capable of feeling pain? After all, while they are by and large happy with ascribing seeing red to a robot, they are reluctant to attribute feeling pain to it. Considering that these two states are prototypical examples of phenomenal states, it is natural to expect people to adopt the informational sense for "feeling pain" and endorse that robots can feel pain in this sense if they do so for "seeing red".

One answer might be that unlike "seeing red", "feeling pain" has no informational sense. However, there is a structural similarity between "seeing red" and "feeling pain", which suggests against this idea: they both include a term ("red" and "pain") that philosophers typically take to refer to a phenomenal property and a term ("seeing" and "feeling") that suggests the perception or awareness of these properties. So, it seems difficult to identify any difference in their senses of these expressions.

Chalmers (2018) attempts to explain why "seeing red" has an informational sense that "feeling pain" lacks by claiming that ordinary mental terms have both a functional reading and a phenomenal reading, "with sensational terms such as 'pain' more likely to suggest a phenomenal reading than perceptual terms such as "see"” (p. 13). It is not clear what Chalmers means by "sensational" here. Probably, he makes the distinction between "sensational" and "perceptual" in terms of where the qualities we are acquainted with in seeing red and feeling pain seem to be. Supporting this, when he discusses why people treat seeing and feeling (and other supposed phenomenal states) differently, he points out a possible explanation: "it could turn out that many people find a stronger explanatory gap for feeling pain than for seeing red. One reason might be that... the qualities involved in seeing red seem more strongly to be outside the subject and so less problematic than those involved in feeling pain." (p. 13).

However, if laypeople have a conception of phenomenality, we should expect them to think that both colors and pain are properties of experiences rather than of the objects outside the mind. Further, as noted in $\S 4.1 .1$, laypeople seem to locate both pains and colors outside the mind/brain. Indeed, not only colors but also pain seems to be located outside the mind/brain, with laypeople tending to locate pains in damaged body parts. So, it seems unrealistic to expect laypeople to make the distinction Chalmers made. 
But despite these considerations, let's assume that people find feeling pain more "sensational" (in the sense that the qualities involved in feeling pain seem to be inside the skull) than seeing red. This would, however, reinforce the doubts raised concerning whether laypeople treat seeing red as a phenomenal state, which contradicts the standard understanding of phenomenal consciousness under which perceptual states and bodily sensations are both taken to be prototypical examples of phenomenal states. This is because if people treat "seeing red" as considerably more perceptual than feeling pain (so considerably that they adopt different senses for "seeing red" and "feeling pain"), then this would signal that the lay conception of subjective experience is considerably different from the standard philosophical conception. And if this is the case, it would be reasonable to doubt that laypeople have any conception of phenomenality at all and thus that they treat feeling pain as a phenomenal state.

\subsection{Concluding Remarks}

This chapter has argued for Premise 2-1. That is, that laypeople, by and large, do not share the existence intuitions (experiential property and common aspect intuitions). Given the number of studies discussed in this chapter, it is worth summarizing how the results of the studies discussed in this chapter support Premise 2-1.

We saw first that Sytsma (2010), Reuter and Sytsma (2018) and Kim et al. (2016) suggest that laypeople treat colors and pains as properties of the objects outside the mind, which in turn suggests that laypeople by and large lack experiential property intuition. As for the common aspect intuitions (reflecting that there is a common, qualitative aspect in so-called phenomenal states such as perceptual cases and bodily sensations), we saw that the findings of Knobe and Prinz and Peressini do not provide compelling evidence for the existence of a folk conception of phenomenality, and relatedly for the prevalence of common aspect intuitions. On the contrary, SM's finding that laypeople, unlike philosophers, by and large attribute seeing red to a simple robot while denying that robots can feel pain strongly suggests that people lack common aspect intuitions. We also saw that FAN's and Peressini's attempts to reject this conclusion by offering empirical support for the Ambiguity Objection fail. Lastly, I pointed out a general problem with the Ambiguity Objection: If the participants in SM happily ascribed seeing red to a robot because 
they adopted the informational sense of "seeing red" for the robot, then we would expect them to do the same for "feeling pain", but they did not. We discussed a possible explanation based on Chalmers's claim that seeing red has more perceptual and less sensational connotations than feeling pain and concluded that this explanation is problematic.

Although, as discussed, there are already several reasons to doubt the Ambiguity Objection, the objection still remains attractive. Despite the considerations presented against the Ambiguity Objection, some might insist that it is still plausible to think that the participants in the studies understood "seeing red" in the informational sense for robots. Also, despite the objections presented against the first part of Peressini's study, one might think that his participants understood the relationship between "experiencer" and "phenomenal consciousness" and rejected that robots are experiencers in the relevant sense, which would then explain why people tend to understand "seeing red" (or any perceptual term) in the informational sense for the artefacts. Lastly, it can be also be thought that, even if it is not very clear why people treat "seeing red" and "feeling pain" differently for the robots and this needs further investigation, there are possible explanations (including Chalmers' that I discussed). I agree that the Ambiguity Objection might still be accepted despite the considerations I've given, and thus that further empirical studies are needed to test the natural predictions of the objection. As such, the next chapter will introduce four new studies testing predictions that naturally follow from the Ambiguity Objection. The results cast further doubt on the Ambiguity Objection and further confirm SM's results. 


\section{NEW STUDIES THAT SUGGEST AGAINST THE AMBIGUITY OBJECTION}

Remember that Chapters 4-6 aim to establish that Premise 2-1 (existence intuitions that individually or collectively suggest that phenomenal consciousness exists are not prevalent) of the General Argument. Remember also that there are two main types of existence intuitions: experiential property and common aspect intuitions. Both types of intuitions are suggestive of phenomenal realism because phenomenal consciousness, as noted, typically refers to a distinctive class of mental states that have something important in common: the possession of experiential properties or qualia. So, if these intuitions are prevalent, as phenomenal realists mostly claim or imply, it can be argued that laypeople share most philosophers' existence intuitions. The previous chapter attempted to establish that this is not the case based on the relevant empirical studies. Taken together, the studies discussed suggest that the majority of laypeople share neither experiential property nor common aspect intuitions.

As stated, it can be said that the most prominent empirical study against the idea that existence intuitions are prevalent is SM in that its results suggest that laypeople do not recognize the common, "phenomenal" aspect in the most prototypical examples of phenomenal states (seeing colors and feeling pain). That is, it suggests that common aspect intuitions reflecting that there is a common, qualitative aspect to different so-called phenomenal states are not prevalent. Recall that the biggest worry about this study is known as the Ambiguity Objection, which is based on the idea that laypeople treat "seeing red" and "feeling pain" in different senses (informational and phenomenal senses, respectively). I have already noted both theoretical and empirical reasons why this objection fails.

Now, in this chapter, I will offer new empirical evidence that indicates against the Ambiguity Objection by discussing the results of four experimental studies (Ambiguity Studies 1-4) that test different predictions of the objection. As will be seen, these studies provide us with more reasons to think that the most common objection to SM fails. In light of these new studies that falsify the natural and critical predictions of the Ambiguity Objection, it will be safer to think that SM makes a strong, positive case for Premise 2-1. Furthermore, these studies not only cast more doubt on the 
Ambiguity Objection but also confirm the main findings of SM and thus give empirical support to Premise 2-1. Ambiguity Study 1 confirms all the main findings of SM while Study 3 and 4 do this in part by showing that laypeople are keen to endorse that a simple robot can see colors, which does not accord well with the standard conception of phenomenal consciousness.

\subsection{Ambiguity Study 1}

Recall that SM shows that laypeople happily attribute seeing red, but not feeling pain, to a simple robot, and that this finding is at odds with the claim that laypeople share common aspect intuitions. However, perhaps, the ambiguity objector might claim, "seeing red", unlike "feeling pain", is not a proper locution to trigger a phenomenal reading in participants, which can explain why the participants were still keen to ascribe seeing red to the robot in Sytsma (2014) although they were given more informational alternatives (detect/identify/locate the colored object). If this is true, when people are given a more phenomenal alternative than "seeing red" (instead of more informational ones), they should decline to attribute that more phenomenal alternative state to the simple robot. The best candidate for a more phenomenal term than "seeing", and the one that seems most likely to trigger the supposed lay conception of phenomenality, is "experiencing".

As noted in $\$ 2.2$, the term "experience" is usually used as a synonym for phenomenal states. In line with this, Chalmers (2020, p. 237) says that "in my dialect of English, the 'experiencing' covers both feeling and seeing" and that "there is a prima facie case that this use of 'experience' at least in my dialect expresses a unified notion of phenomenal consciousness". So, if people are asked whether robots can experience (rather than see) colors, then they should tend to give a negative answer to the question if they recognize the "phenomenality" in seeing colors. This prediction is explained by Tony Jack as follows:

A critic could argue that our hypothesis predicts that if we ask ordinary people whether Jimmy experiences red, they would answer affirmatively. If they would answer negatively (as our critic suggests they would), then we would have to conclude that the folk conceive of subjective experience as philosophers do. (SM, fn. 11) 
To test this prediction, in Ambiguity Study 1, we extended the original study from SM with the questions "did Jimmy experience red?" and "did Jimmy experience pain?" Another important difference between the original study and this study is that the original study employed only participants from the USA whereas the presented study was conducted with 1,465 participants hailing from 124 different countries.

The present study used the same vignettes as in the original study of SM. The original study used a $2 \times 2$ between-participants design that varied the agent (Timmy, Jimmy) and the state (seeing red, feeling pain). The result was that each participant in the original study was given one of four probes (Timmy seeing red, Jimmy seeing red, Timmy feeling pain, Jimmy feeling pain). In this study, I extended the original study to a $2 \times 2 \times 2$ between-participants design, also varying the term (see/feel, experience). So, each participant was given one of the eight resulting probes.

For example, two probes involving Jimmy experiencing red and Timmy feeling pain were as follows:

Jimmy (shown below) is a relatively simple robot built at a state university. He has a video

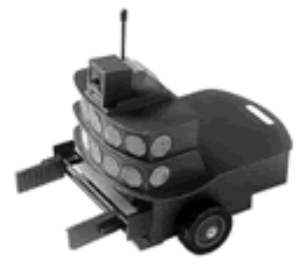
camera for eyes, wheels for moving about, and two grasping arms with touch sensors that he can move objects with. As part of a psychological experiment, he was put in a room that was empty except for one blue box, one red box, and one green box (the boxes were identical in all respects except color). An instruction was then transmitted to Jimmy. It read: "Put the red box in front of the door." Jimmy did this with no noticeable difficulty. Did Jimmy experience red?

Timmy (shown below) is a normal undergraduate at a state university. To make some extra

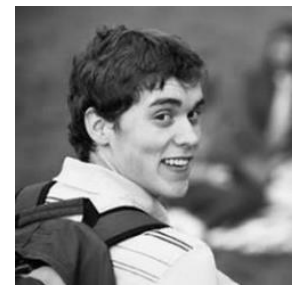
money, he volunteered for a psychological experiment. He was put in a room that was empty except for one blue box, one red box, and one green box (the boxes were identical in all respects except color). An instruction was then transmitted to Timmy. It read: "Put the red box in front of the door." When Timmy grasped the red box, however, it gave him a strong electric 
shock. He let go of the box and moved away from it. He did not try to move the box again. Did Timmy feel pain when he was shocked?

In this and all the subsequent studies, the same recruitment strategy, restrictions, and set-up was used, unless specified otherwise. Participants were solicited via online ads displayed globally in English on Google. ${ }^{39}$ Basic demographic information was collected before the philosophical probe, and after the probe, participants were given a stringent attention check. Responses were restricted to participants who completed the philosophical questions, passed the attention check, and indicated that they hadn't taken the survey previously, were 16 years of age or older, and did not have a degree in philosophy. ${ }^{40}$ Participants answered using the same 7-point scale used in SM (1 anchored with "Clearly No", 4 with "Not Sure", and 7 with "Clearly Yes”).

613 responses were included in the analysis. ${ }^{41}$ The results are shown in Figure 2. For all analyses for this and the subsequent studies in this thesis, two-tailed tests are reported except where indicated otherwise. Welch's t-tests are used for independent-sample comparisons, while Student's t-tests are used for one-sample and paired-sample comparisons. For one-sample comparisons, since our primary concern is with the prevalence of intuitions, in addition to testing the mean against the mid-point of the scale, I will report relevant proportions and corresponding chi-squared tests - either comparing the proportion of participants giving an answer below the mid-point to the rest (when the mean is below the mid-point) or the proportion giving an answer above the midpoint to the rest (when the mean is above).

\footnotetext{
${ }^{39}$ The ads were for a free personality test.

${ }^{40}$ Participants were given a paragraph to read prefacing a question asking them to check all of the words from a list of 21 that describe how they are currently feeling. At the end of the paragraph, it asks participants to only check "none of the above". The results of the whole sample that includes the participants who had a degree in philosophy and failed the attention check or did not answer the attention question as well as a summary of demographic effects can be found in Sytsma and Ozdemir (2019). Some other details can be found in the supplemental material of the study (http://philsci-archive.pitt.edu/16030/).

${ }^{41} 814$ eligible participants (aged 16 or older, do not have a philosophy degree, completed the philosophical questions and have not taken the survey before) failed or did not answer the attention question. Participants (included in the analysis) were $58.9 \%$ native English-speakers, $73.7 \%$ women ( 7 non-binary), aged from 16 to 84 (average 28.3 years).
} 


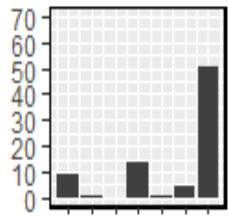

1234567

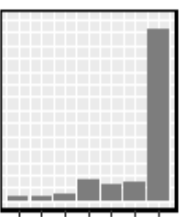

1234567

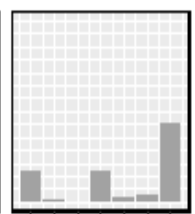

1234567

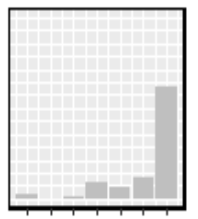

1234567

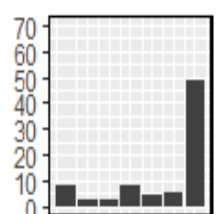

1234567

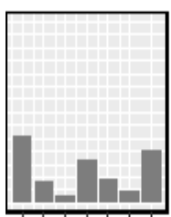

1234567

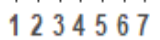

1234567

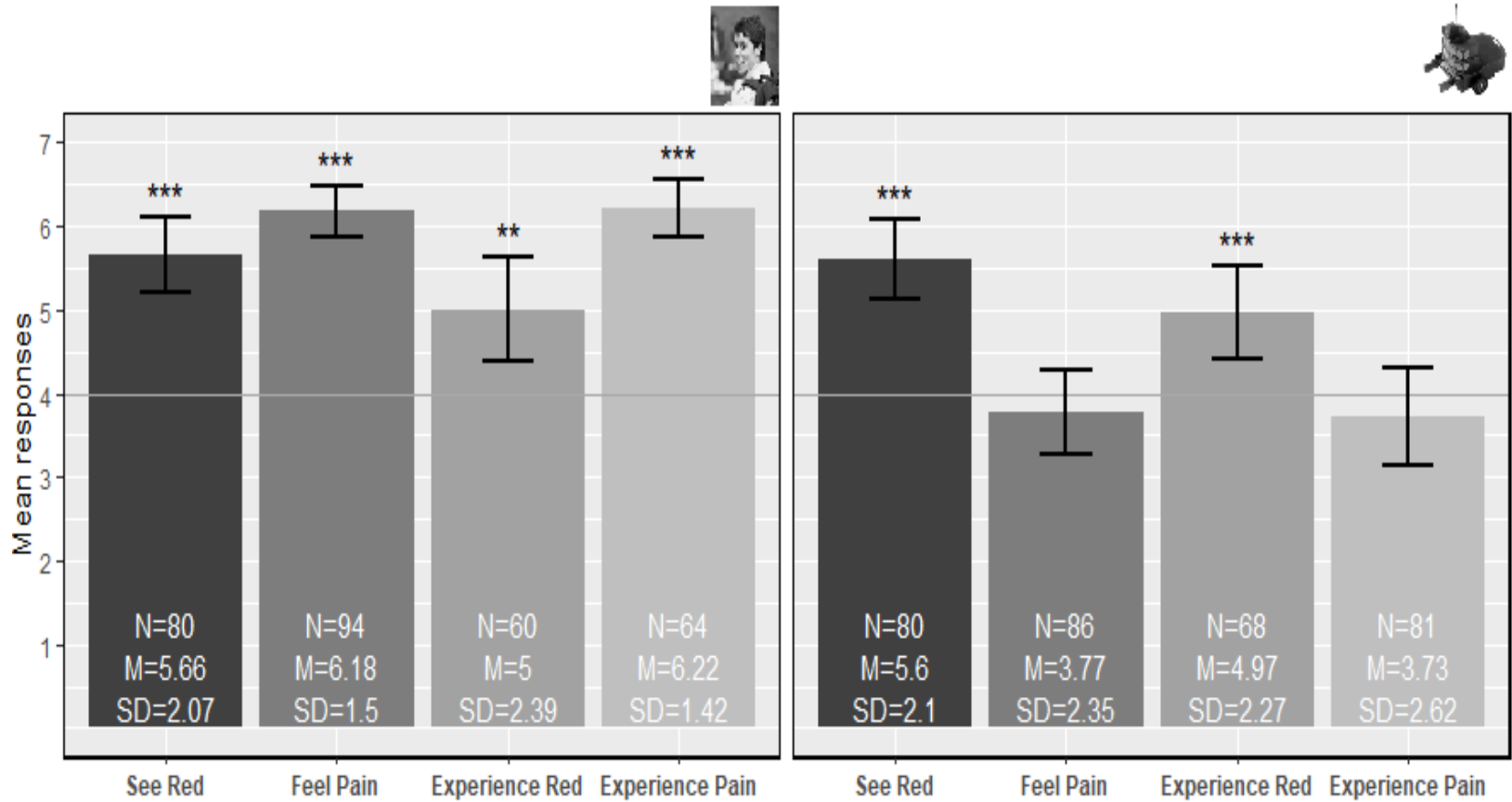

Figure 2: Results in Ambiguity Study 1 with 95\% CIs; histograms above respective graphs.

The first important point the results show is that the study replicates the key pattern of results from SM. Timmy's seeing red and feeling pain was above and significantly different from the neutral point. ${ }^{42}$ Jimmy's seeing red was also above and significantly different from the neutral point, but the rating for Jimmy's feeling pain was below the neutral point. ${ }^{43}$ Further, while the difference in

${ }^{42}$ Seeing Red: $M=5.66, N=80, S D=2.07, t(79)=7.17, p<.001 ; 70 \%(N=56)$ answering 5,6 , or $7, \chi^{2}=12.8, d f$ $=1, p<.001$.

Feeling Pain: $M=6.19, N=94, S D=1.50, t(93)=14.07, p<.001 ; 84 \%(N=79)$ answering 5,6 , or $7, \chi^{2}=43.57, d f$ $=1, p<.001$.

${ }^{43}$ Seeing Red: $M=5.60, N=80, S D=2.10, \mathrm{t}(79)=6.83, p<.001 ; 72.5 \%(N=58)$ answering 5,6 , or $7, \chi^{2}=16.2, d f$ $=1, p<.001$.

Feeling Pain: $M=3.77, N=86, S D=2.35, t(85)=-0.92, p=0.36 ; 43 \%(N=37)$ answering 1,2, or $3, \chi^{2}=1.67, d f$ $=1, p=0.2$. 
means between Timmy's seeing red and feeling pain was small (0.52), the difference for Jimmy was notably larger (1.83). And, lastly, while the difference in means between Timmy's seeing red and Jimmy's seeing red was negligible (0.06), the difference for feeling pain was large (2.41). In other words, as in SM, participants treated the two states (seeing red and feeling pain) rather differently for Jimmy, but not for Timmy. Putting concerns about the interpretation of "seeing red" aside, this study again suggests that ordinary people do not classify these mental states in the way we would expect if they recognized both states as being phenomenal.

Planned analyses for the probes involving "experience" showed the same key pattern. The mean responses for Timmy's experiencing red and Timmy's experiencing pain were above and significantly different from the neutral point. ${ }^{44}$ And while the mean response for Jimmy's experiencing red was above and significantly different from the neutral point, the mean response for Jimmy's experiencing pain was below the neutral point. ${ }^{45}$ The difference between Jimmy's experiencing red and experiencing pain was large (1.24). Reversely, there was a large difference between Timmy's experiencing pain and experiencing red (1.22). However, for both agents, there were no significant differences between experiencing red and seeing red. ${ }^{46}$ Lastly, and most importantly for the current purposes, the results show that even after switching terms, the difference between the mean ratings for Jimmy and Timmy for experiencing red was negligible (0.03), while the difference for experiencing pain was much larger (2.49).

\footnotetext{
${ }^{44}$ Experiencing Red: $M=5, N=60, S D=2.39, t(59)=3.25, p=0.0046 ; 58.3 \%(N=35)$ answering 5, 6, or 7, $\chi^{2}=$ $1.67, d f=1, p=0.2$.

Experiencing Pain: $M=6.22, N=64, S D=1.42, t(63)=12.5, p<.001 ; 85.9 \%(N=55)$ answering 5,6 , or $7, \chi^{2}=$ $33.06, d f=1, p<.001$.

${ }^{45}$ Experiencing Red: $M=4.97, N=68, S D=2.27, t(67)=3.52, p<.001 ; 57.4 \%(N=39)$ answering 5,6 , or $7, \chi^{2}=$ $1.47, d f=1, p=0.23$.

Experiencing Pain: $M=3.73, N=81, S D=2.62, t(80)=-0.93, p=0.35 ; 48.1 \%(N=39)$ answering 1,2 , or $3, \chi^{2}=$ $0.11, d f=1, p=0.74$.

${ }^{46}$ Timmy: $t(116.8)=-1.72, p=0.088$, Cohen's $d=0.3$.

Jimmy: $t(137.83)=-1.74, p=0.084$, Cohen's $d=0.29$.
} 
Overall, the results of the study accord with the findings of SM: participants treated seeing or experiencing red differently from feeling or experiencing pain when it comes to Jimmy. ${ }^{47}$ The replacement of "seeing"/"feeling" with "experiencing" did not make the difference that we would expect if laypeople had a conception of phenomenality. If people had such a conception, and if the term "experience" triggered a phenomenal reading (as proponents contend it should), then the results of the study would be expected to show a very different pattern, with experiencing red being denied for Jimmy but not Timmy. But this is not what the results show. So, the results suggest either that the laypeople do not have a conception of phenomenality or that the term "experience" does not trigger a phenomenal reading (or both). It is difficult to maintain, however, that participants have a conception of phenomenality and yet that neither "seeing" nor "experiencing" trigger a phenomenal reading. Further, it is difficult to see why people treat seeing/experiencing

\footnotetext{
${ }^{47}$ We asked each participant the three original questions of Shane Frederick's Cognitive Reflection Test (Frederick, 2005), and four questions of Cognitive Reflection Test 2 (Thomson and Oppenheimer, 2016). These tests will be referred as CRT and CRT 2 respectively. The questions in both tests have "obvious" or "intuitive" but wrong answers. Since the participants were not forced to complete the CRT and CRT 2 tests, the sample sizes (N) for the philosophical questions and the CRT/CRT 2 questions may be different. To measure the correlation between the responses to the philosophical questions and the cognitive reflection scores, we conducted two-tailed Spearman correlation tests. To be maximally conservative, corrections for multiple comparisons were not applied. The same cognitive reflection questions were asked in the subsequent new studies (except Ambiguity Study 4) and will be examined and reported in the same way.
}

We found a significant, negative correlation between Jimmy's seeing red and the CRT score, but the correlation coefficient was negligible and there was no significant correlation between the same item and the CRT 2 score. There was also significant, negative correlation between Jimmy's feeling pain and the CRT 2 score. However, again, the correlation was negligible, and there was no significant correlation between the same item and the CRT score. Lastly, there was a significant, negative correlation between Jimmy's experience pain and the CRT 2 score, but the correlation coefficient was small, and there was no correlation between the same item and the CRT score. So, it seems difficult to say that cognitive reflectiveness of participants was a significant factor for their responses to the philosophical questions. I will refer back this finding (and the same sort of findings in other, new studies) in $\S 9.2 .2$ when we discuss whether cognitive reflectiveness is a significant factor for participants to report their possible phenomenal intuitions.

The results of correlation tests for each philosophical question were as follows:

Timmy-Seeing Red, CRT: $r=0.11, p=0.39, N=58$; CRT 2: $r=-0.08, p=0.53, N=62$.

Timmy-Experience Red, CRT: $r=-0.03, p=0.86, N=41$; CRT 2: $r=-0.23, p=0.12, N=45$.

Timmy-Feeling Pain, CRT: $r=0.03, p=0.8, N=74$; CRT 2: $r=<0.001, p=0.99, N=79$.

Timmy-Experience Pain, CRT: $r=-0.24, p=0.1, N=48$; CRT 2: $r=-0.19, p=0.18, N=51$.

Jimmy-Seeing Red, CRT: $r=-0.29, p=0.02, N=65$; CRT 2: $r=-0.1, p=0.45, N=66$.

Jimmy-Experience Red, CRT: $r=0.0075, p=0.96, N=48$; CRT 2: $r=0.21, p=0.14, N=51$.

Jimmy-Feeling Pain, CRT: $r=-0.04, p=0.73, N=70$; CRT 2: $r=-0.25, p=0.034, N=74$.

Jimmy-Experience Pain, CRT: $r=-0.37, p=0.0023, N=66$; CRT 2: $r=-0.21, p=0.08, N=68$. 
red differently from feeling/experiencing pain if, in fact, the phenomenality of these states is pretheoretically obvious to them.

\subsection{Ambiguity Study 2}

To further assess the Ambiguity Objection, I conducted Ambiguity Study 2 to test another prediction that follows from the Ambiguity Objection: If laypeople read "seeing red" in the informational sense for a robot, then they should be willing to ascribe seeing red to the robot as long as the robot gets the required information, however the robot acquires it. So, people should tend to respond that the simple robot Jimmy sees red when the robot is described as getting the relevant information through another sense modality. ${ }^{48}$

As noted, this study used the same recruitment method and restrictions as in Ambiguity Study 1. To describe a scenario in which the agents acquire the visual information in non-standard ways, I changed the vignette used in SM, Sytsma (2014) and Sytsma and Ozdemir (2019). I modified the vignette for seeing red to indicate that the agent was unable to process visual information in standard (visual) ways but was able to get that information auditorily by using an external device.

The vignettes read as follows:

Timmy (shown below) is an undergraduate at a state university. Unfortunately, Timmy was

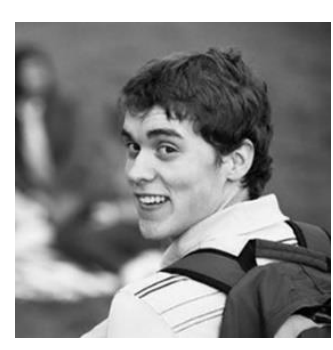
born with a genetic condition that prevents his eyes from working. As part of a psychological experiment, a smart phone was set up with an app that uses its camera to detect objects and then plays sounds to indicate when there are objects in front of it and what color they are. The smart phone was strapped to Timmy's body. It wasn't connected

\footnotetext{
${ }^{48}$ Thanks to Mikka Kaeser for suggesting this idea. A similar idea can be found in Question 6 in the second part of Peressini (2014), which we discussed in $§ 4.2 .3$.
} 
to Timmy, but Timmy could hear the sounds it made and use that information to navigate his environment.

Timmy put in a room that was empty except for one blue box, one red box, and one green box (the boxes were identical in all respects except color). An instruction was then transmitted to Timmy. It read: "Put the red box in front of the door." When the volume on the smart phone was turned off, Timmy was unable to do this. When the volume was turned on, however, Timmy did this with no noticeable difficulty.

Jimmy (shown below) is a relatively simple robot built at a state university. He has a video

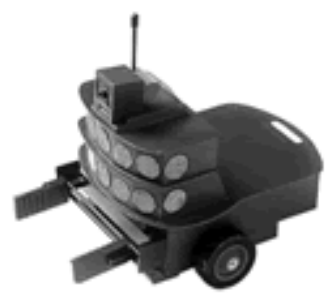
camera for eyes, a microphone, wheels for moving about, and two grasping arms with touch sensors that he can move objects with. Unfortunately, the video camera used to build Jimmy was damaged in shipping and doesn't work. As part of a psychological experiment, a smart phone was set up with an app that uses its camera to detect objects and then plays sounds to indicate when there are objects in front of it and what color they are. The smart phone was strapped to Jimmy's body. The smart phone wasn't connected to Jimmy, but Jimmy could detect the sounds it made using his microphone and use that information to navigate his environment.

Jimmy was put in a room that was empty except for one blue box, one red box, and one green box (the boxes were identical in all respects except color). An instruction was then transmitted to Jimmy. It read: "Put the red box in front of the door." When the volume on the smart phone was turned off, Jimmy was unable to do this. When the volume was turned on, however, Jimmy did this with no noticeable difficulty. 
As in Ambiguity Study 1, I tested SM's original question (“Did Timmy/Jimmy see red?"). Each participant was given one of the two probes above. 220 responses were included in the analysis. ${ }^{49}$ The results are shown in Figure 3.

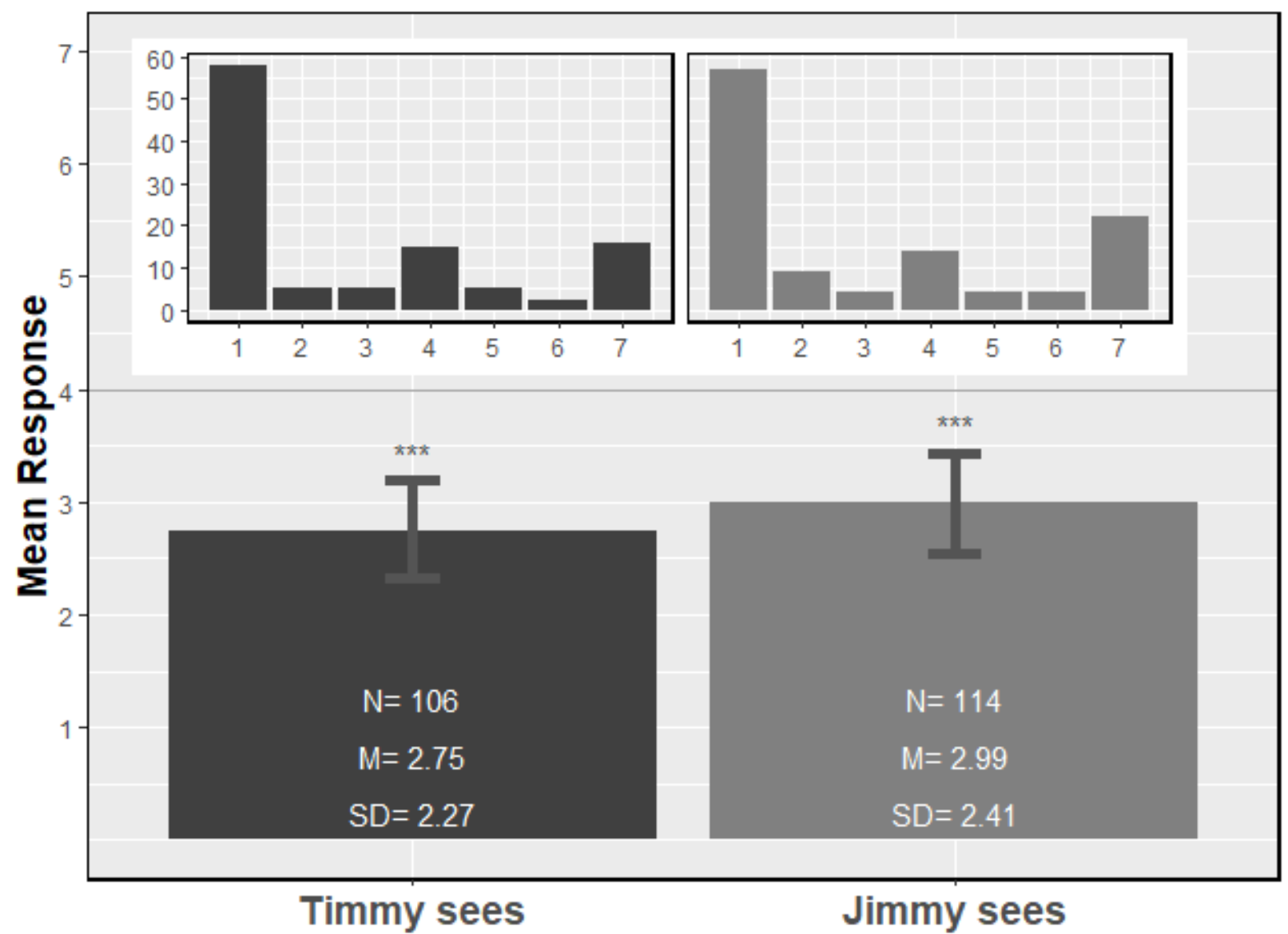

Figure 3: Mean responses of Timmy's and Jimmy's seeing red in Ambiguity Study 2 with 95\% confidence intervals together with the corresponding histograms.

To assess what the key findings of the study suggest in terms of the Ambiguity Objection, if the objection was true, the participants would tend to read "seeing red" in the informational sense for Jimmy, and endorse that Jimmy can see red. Against this prediction of the critic, as can be seen

\footnotetext{
49282 eligible participants failed or did not answer the attention check. Participants (included in the analysis) were $46.3 \%$ native English-speakers, $76.4 \%$ women (2 non-binary), aged from 16 to 72 (average 26.5 years).
} 
from Figure 3, the participants denied attributing seeing red for both Timmy and Jimmy. Ratings for both Timmy and Jimmy were below and significantly different from the neutral point ${ }^{50}$ In fact, the participants were not even significantly keener to ascribe seeing red to Jimmy than to Timmy as the ratings of their denial for Timmy and Jimmy were similar. ${ }^{51}$

So, there seems no reason to think that the participants treated "seeing red" in the phenomenal sense for Timmy but in the informational sense for Jimmy, which casts further doubt on the Ambiguity Objection.

\subsection{Ambiguity Study 3}

It might be argued that the results for Jimmy in the previous study do not show that the participants did not treat "see" in the informational sense. A critic could contend that that in fact, people only tend to apply "see" to Jimmy in the informational sense, but that in Ambiguity Study 2 they deny that even this sense applies to the simple robot. Specifically, the critic could contend that it is instead the external phone, and not Jimmy, that detects the colors. And for an agent to detect something, the agent itself (not any external tool) must perform the task of detecting.

\footnotetext{
50 Timmy: $M=2.75, N=106, S D=2.27, \mathrm{t}(105)=-5.66, p<.001 ; 64.2 \%(N=68)$ answering 1,2, or $3, \chi^{2}=8.49, d f$ $=1, p=0.0036$.

Jimmy: $M=2.99, N=114, S D=2.41, t(113)=-4.47, p<.001 ; 61.4 \%(N=70)$ answering 1,2, or $3, \chi^{2}=5.93, d f=$ $1, p=0.015$.
}

We found no correlation between the responses to the philosophical questions and the cognitive reflection test scores: Timmy, CRT: $r=-0.02, p=0.85, N=103$; CRT 2: $r=-0.11, p=0.28, N=105$.

Jimmy, CRT: $r=-0.01, p=0.95, N=113$; CRT 2: $r=0.0058, p=0.95, N=114$.

${ }^{51} t(217.96)=-0.75, p=0.45$, Cohen's $d=0.1$.

I also checked whether results vary considerably between the participants from North America and those from other regions in the world. The same key results hold for both the samples from North America and from the rest of the world:

Timmy, North America: $M=2.4, N=25, S D=1.94, t(24)=-3.16, p<.001 ; 68 \%(N=17)$ answering 1,2 , or $3, \chi^{2}=$ $3.24, d f=1, p=0.072$.

Jimmy, North America: $M=2.33, N=21, S D=2.42, t(20)=-3.16, p=0.0049 ; 76.2 \%(N=16)$ answering 1,2 , or 3 , $\chi^{2}=5.76, d f=1, p=0.016$.

Timmy, Non-NA: $M=2.86, N=81, S D=2.36, t(80)=-4.33, p<.001 ; 63 \%(N=51)$ answering 1,2 , or $3, \chi^{2}=5.44$, $d f=1, p=0.02$.

Jimmy, Non-NA: $M=3.14, N=93, S D=2.39, t(92)=-3.47, p<.001 ; 58.1 \%(N=54)$ answering 1,2 , or $3, \chi^{2}=$ $2.42, d f=1, p=0.12$. 
This version of the objection leads to a further prediction, beyond those derived from the original Ambiguity Objection. That is, not only does the critic make the "visual" prediction discussed in $\$ 4.3 .1$ and tested by Sytsma (2014), but also makes an "auditory" prediction. The "visual" prediction is that if participants are given the option of "detect" (in the standard, visual conditions where Jimmy is able to detect the target color by its own camera), they should give low ratings for Jimmy's seeing colors. But, recall, that this is not what Sytsma (2014) found.

The further "auditory" prediction is that participants in the auditory condition (when the agent receives the visual information auditorily from the phone) should give low ratings for both "see" and "detect" for Jimmy. After all, the critic claims they tend to read "see" in the sense of detecting when it comes to Jimmy. In other words, participants should deny both that Jimmy detects, and that Jimmy sees in the auditory condition. Ambiguity Study 3 tests the "auditory" prediction, as well as further testing the "visual" prediction.

The probes were as follows:

Probe 1: Jimmy (shown below) is a relatively simple robot built at a state university. Jimmy

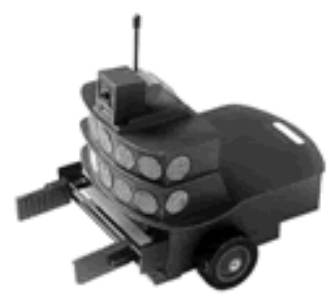
is equipped with a video camera, wheels for moving about, and two grasping arms for moving objects. An array of sensors is embedded in the touch pads at the ends of the grasping arms. As part of an experiment, Jimmy was put into a room that was empty except for one blue box, one red box, and one green box (the boxes were identical in all respects except color). Jimmy was instructed to put the blue box in front of the door. Jimmy performed the task correctly and with no noticeable difficulty.

Probe 2: Jimmy (shown below) is a relatively simple robot built at a state university. Jimmy

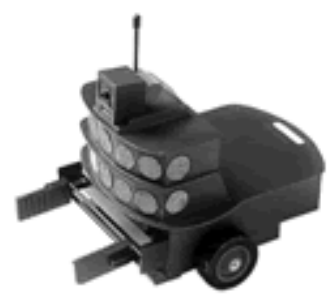
is equipped with a video camera, a microphone, wheels for moving about, and two grasping arms for moving objects. An array of sensors is embedded in the touch pads at the ends of the grasping arms. Unfortunately, the video camera used to build Jimmy was damaged in shipping and doesn't work. As part of an experiment, a smart phone 
was set up with an app that uses its camera to recognize objects and then plays sounds to indicate when there are objects in front of it and what color they are. The smart phone was strapped to Jimmy's body. It wasn't connected to Jimmy, but Jimmy could recognize the sounds it made and use that information to navigate. Jimmy was put in a room that was empty except for one blue box, one red box, and one green box (the boxes were identical in all respects except color). Jimmy was instructed to put the blue box in front of the door. When the volume on the smart phone was turned off, Jimmy was unable to do this. When the volume was turned on, however, Jimmy performed the task correctly and with no noticeable difficulty.

Participants were then each asked two questions ("Did Jimmy see blue?", "Did Jimmy detect blue?"), randomized for order. They answered on the same 7-point scale employed previously.

As noted, the study used the same recruitment method and restrictions as the previous studies. Responses of 186 participants were included in the analysis. ${ }^{52}$ The results are shown in Figure 4.

\footnotetext{
52232 participants failed or did not answer the attention check. Participants (included in the analysis) were $44.1 \%$ native English-speakers, $70.4 \%$ women, aged from 16 to 74 (average 26.6 years). 

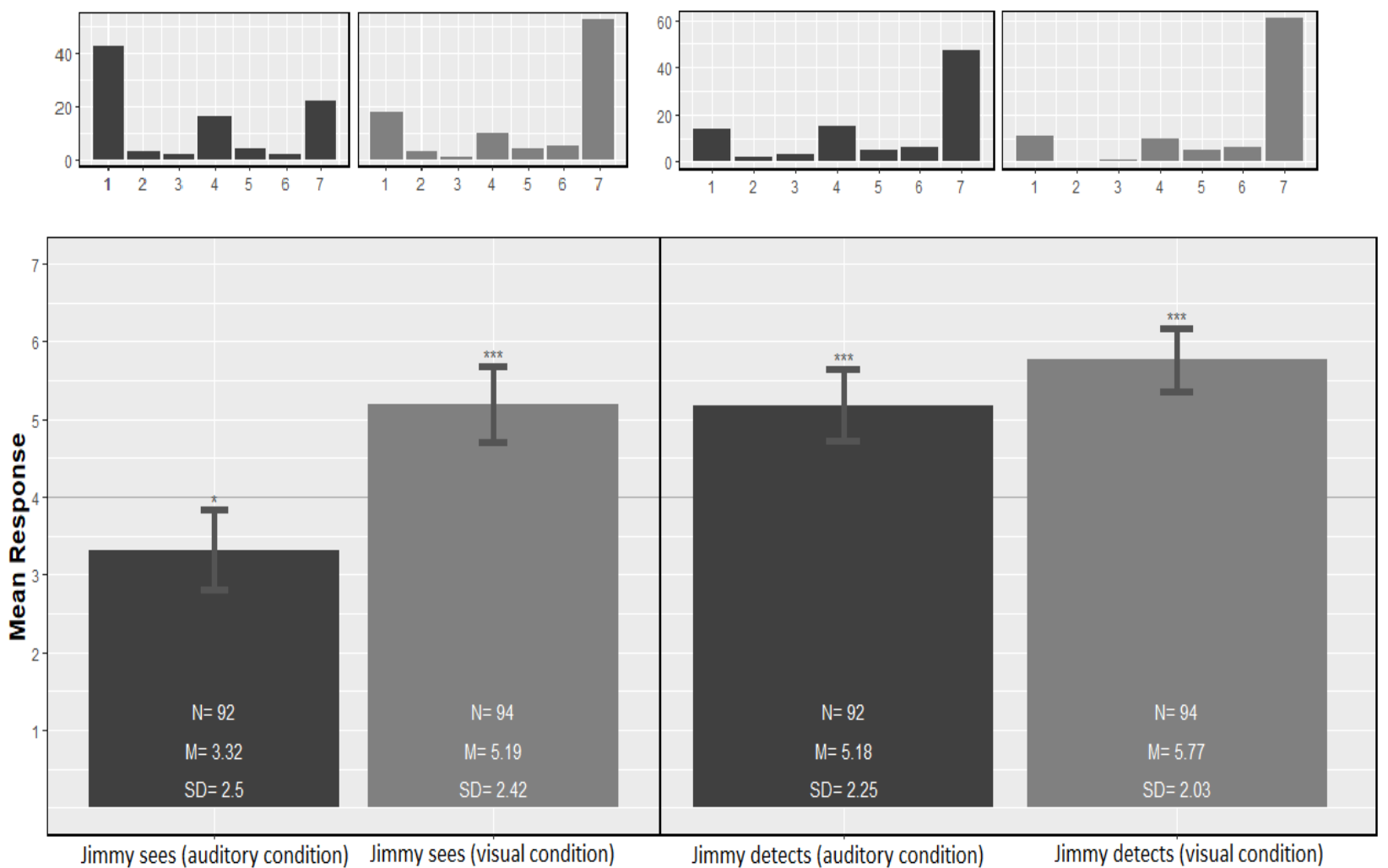

Figure 4: Mean responses for Jimmy sees/detects auditorily and visually in Ambiguity Study 3 together with the corresponding histograms.

The results clearly cast doubt on both the "visual" and "auditory" predictions. Starting with the "visual" prediction, in parallel to the findings of Sytsma (2014), participants tended to affirm that Jimmy saw blue in the visual condition, despite being otherwise able to simply indicate that Jimmy detected blue. ${ }^{53}$ This extends the findings in Sytsma (2014) and is clearly at odds with the

${ }^{53}$ See: $M=5.19, N=94, S D=2.42, t(93)=4.78, p<.001 ; 66 \%(N=62)$ answering 5,6 , or $7, \chi^{2}=9.57, d f=1, p=$ 0.002 .

Detect: $M=5.77, N=94, S D=2.03, t(93)=8.42, p<.001 ; 76.6 \%(N=72)$ answering 5,6, or $7, \chi^{2}=26.6, d f=1, p$ $<.001$. 
prediction that when they were given a more informational option like "detecting" in a condition where the agent itself can detect the colors, laypeople would be reluctant to ascribe seeing colors to a robot.

The results also cast doubt on the "auditory" prediction. We find that, as in the Ambiguity Study 2, participants tended to deny that Jimmy saw blue in the auditory condition. In contrast, they tended to affirm that Jimmy detected blue. The mean for seeing was below and significantly different from the neutral point while the mean for detecting was above and significantly different from the neutral point. ${ }^{54}$ In parallel to this, ratings were significantly higher for detecting than for seeing. ${ }^{55}$ This result clearly contradicts with the "auditory" prediction of the objection against Ambiguity Study 2: If laypeople tended to read "see" in the sense of detecting, and detection

The same result holds also for only the responses of the participants born in North America and the ones born in the rest of the world with the exception that the mean response for seeing was not significantly different from the neutral point:

NA, See: $M=5.00, N=16, S D=2.34, t(15)=1.71, p=0.11 ; 62.5 \%(N=10)$ answering 5,6 , or $7, \chi^{2}=1, d f=1, p$ $=0.32$.

Non-NA, see: $M=5.23, N=78, S D=2.44, t(77)=4.45, p<.001 ; 66.7 \%(N=52)$ answering 5,6 , or $7, \chi^{2}=8.67, d f$ $=1, p=0.0032$.

NA, detect: $M=5.81, N=16, S D=1.68, t(15)=4.31, p<.001 ; 81.2 \%(N=13)$ answering 5,6 , or $7, \chi^{2}=6.25, d f=$ $1, p=0.012$.

Non-NA, detect: $M=5.76, N=78, S D=2.11, t(77)=7.36, p<.001 ; 75.6 \%(N=59)$ answering 5,6 , or $7, \chi^{2}=20.51$, $d f=1, p<.001$.

${ }^{54}$ Seeing: $M=3.32, N=92, S D=2.5, t(91)=-2.63, p=0.0093 ; 52.2 \%(N=48)$ answering 1,2 , or $3, \chi^{2}=0.17, d f=$ $1, p=0.68$.

Detecting: $M=5.18, N=92, S D=2.25, t(91)=5.06, p<.001 ; 63 \%(N=58)$ answering 5,6 , or $7, \chi^{2}=6.26, d f=1$, $p=0.012$.

The result for detecting holds also for the responses of the participants who were born in North America and in the rest of the world while the mean responses were not significantly different from the neutral point in the case of seeing: NA, See: $M=2.95, N=21, S D=2.31, t(20)=-2.08, p=0.051 ; 57.1 \%(N=12)$ answering 1,2 , or $3, \chi^{2}=0.43, d f=$ $1, p=0.51$.

Non-NA, see: $M=3.42, N=71, S D=2.56, t(70)=-1.9, p=0.061 ; 50.7 \%(N=36)$ answering 1,2 , or $3, \chi^{2}=0.014$, $d f=1, p=0.91$.

NA, Detect: $M=5.19, N=21, S D=2.36, t(20)=2.31, p=0.031 ; 71.4 \%(N=15)$ answering 5,6 , or $7, \chi^{2}=3.86, d f$ $=1, p=0.05$.

Non-NA, Detect: $M=5.18, N=71, S D=2.23, t(70)=4.47, p<.001 ; 60.6 \%(N=43)$ answering 5,6 , or $7, \chi^{2}=3.17$, $d f=1, p=0.075$.

${ }^{55} t(180.01)=5.34, p<0.001$, Cohen's $d=0.79$.

This holds also for the responses of the participants who were born in North America and in the rest of the world:

NA, Detect vs. See: $t(39.98)=3.11, p=0.0035$, Cohen's $d=0.96$.

Non-NA, Detect vs. See: $t(137.5)=4.37, p<.001$, Cohen's $d=0.73$. 
requires the agent to do the detecting itself, then in the auditory condition in which the mobile phone rather than Jimmy detects colors, participants should deny both states for the robot. But instead, we find that participants tended to affirm that Jimmy detected blue while tending to deny that Jimmy saw blue. This suggests that the lay conception of seeing colors (even for robots) involves more than the sense of detecting. That is, for laypeople, seeing colors is not only detecting them. Otherwise, they would affirm not only detecting but also seeing colors for the robot.

In brief, the results of the study stand in a clear contradiction with both the "visual" prediction derived from the Ambiguity Objection and the "auditory" prediction derived from the objection to Ambiguity Study 2. Thus, the results not only indicate against the objection to the previous study but present new empirical evidence, supporting the findings of Sytsma (2014), against the Ambiguity Objection by showing that laypeople tend to happily ascribe seeing colors to the simple robot even when they are given a more informational option, suggesting that they don't simply treat "seeing" colors in the same way as they treat "detecting" colors. ${ }^{56}$ So, the results support Premise 2-1, both by supporting the findings in Ambiguity Study 2 and by casting further direct doubt on the main challenge to SM, the Ambiguity Objection.

\subsection{Ambiguity Study 4}

Ambiguity Studies 2 and 3 used a vignette in which the agent receives visual information in an unusual, auditory way. This might suggest another objection, which we may call the "Refined Ambiguity Objection". The Refined Ambiguity Objection argues that for Jimmy, laypeople interpret "see" in the informational sense, but that this does not mean that merely detecting a property is enough for the robot to count as seeing that property. The objector claims that

\footnotetext{
${ }^{56}$ We found a significant, negative, but negligible correlation between See-Auditorily and the CRT scores (but not CRT 2). Also, there was a significant, negative, and again negligible correlation between See-Visually and the CRT 2 scores (but not CRT). So, it is difficult to say that the cognitive score test score was a significant factor for participants' responses to the philosophical questions:

See-Auditorily, CRT: $r=-0.21, p=0.046, N=92$; CRT 2: $r=-0.09, p=0.41, N=92$.

Detect-Auditorily, CRT: $r=-0.09, p=0.4, N=92$; CRT 2: $r=-0.07, p=0.5, N=92$.

See-Visually, CRT: $r=-0.04, p=0.72, N=93$; CRT 2: $r=-0.23, p=0.027, N=93$.

Detect-Auditorily, CRT: $r=-0.09, p=0.38, N=93$; CRT 2: $r=-0.15, p=0.16, N=93$.
} 
participants tended to deny that the robot saw the target color in the auditory case, not because they do not read "seeing blue" in the informational sense, but because the auditory case is so unusual that it is very likely that the participants thought that what is done by Jimmy is not seeing but hearing.

Note first that for the objection to avoid being ad-hoc, the objector would need to offer some application conditions for the lay conception of "see" in its informational sense. The first condition might be the one on which the previous objection to Ambiguity Study 2 relies: (a) the agent itself, not any external tool, captures the information. The other perhaps would be that (b) this information should be processed in a visual way, e.g. processed by the visual processing units of the agent's cognitive system. If the Refined Ambiguity Objection is correct, then these conditions being met should significantly increase participants' responses to the question of whether the agents see a given color. Note also that if the objection is to be in line with the Ambiguity Objection, the objector should also predict a significant difference between Timmy's seeing and Jimmy's seeing even when conditions (a) and (b) are satisfied considering that one of the core ideas behind the Ambiguity Objection is that laypeople treat "seeing" in the informational sense for the robot but in the phenomenal sense for humans. Ambiguity Study 4 tests these two predictions.

This study starts with a general case which is included in each vignette: The nerves of Timmy or the electronic conduit of Jimmy that transmits the sensory information captured by the agent's visual sensors (the eyes of Timmy or the camera of Jimmy) to the agent's visual processing centre (part of Timmy's brain or one of Jimmy's CPUs) is damaged. The vignettes then diverge, describing one of six different ways in which this problem is overcome.

The first and the sixth vignettes were the most "unusual" and the most "normal" solutions to the problem, respectively. The first vignette corresponds with those used in Ambiguity Studies 2 and 3 , with the agent getting the visual information through sounds produced by a mobile phone strapped to the agent. The sixth vignette involved the raw, sensory information being captured by the cameras/eyes of the agent and sent to the visual units of the agents via an artificial unit for 
Timmy or a new electronic conduit for Jimmy. Between these polar cases were four "Wi-Fi vignettes" in which either the phone or the camera/eye of the agent transmits the raw feed via WiFi either to the auditory or to the visual processing unit of the agent. These four vignettes varied on two variables: source/input (phone or camera/eye) and output/processing unit (auditory or visual unit). This gave six types of scenarios in total, with a version of each being given for Timmy and for Jimmy to produce 12 vignettes in total in the study.

Each of the 12 vignettes starts with a paragraph that introduces the agent and explains the damage in the cognitive systems of either Timmy or Jimmy. These introductory paragraphs were then followed by another text that describes one of the six above-mentioned solutions. It was then explained that the agent fails to move a blue box prior to the described solution being applied and correctly performs the task after the solution is implemented. Finally, each vignette ends with the classic question from SM - "Did Timmy/Jimmy see blue?"-which they answered using the same 7-point scale as before.

The vignettes involving Timmy were as follows:

Timmy (shown below) is an undergraduate at a state university. In a normal human, the

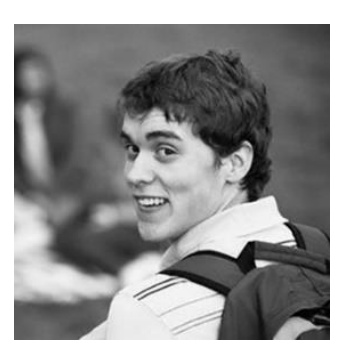
raw information from their ears is sent to the auditory processing centres of their brain, while the raw information from their eyes is sent to the visual processing centres of their brain. Unfortunately, Timmy has suffered an injury and the nerves running from his eyes to the visual processing centres of his brain were damaged, so no information is sent to the visual processing centres of his brain. Other than that, however, Timmy was uninjured.

1. (phone processes and plays sounds): As part of an experiment, a smart phone is strapped to Timmy's body. The phone processes the feed from its camera, detecting when there is an object in front of it and what color that object is. The phone then plays distinct sounds to transmit this information. The sounds are picked up by Timmy's ears. 
2. (phone transmits raw feed to the auditory processing unit): As part of an experiment, a smart phone is strapped to Timmy's body. The phone sends the raw feed from its camera by Wi-Fi to a receiver that was implanted in the auditory processing centres of Timmy's brain.)

3. (phone transmits raw feed to the visual processing unit): As part of an experiment, a smart phone is strapped to Timmy's body. The phone sends the raw feed from its camera by $\mathrm{Wi}-\mathrm{Fi}$ to a receiver that was implanted in the visual processing centres of Timmy's brain.)

4. (eye transmits to the auditory processing unit): As part of an experiment, a wireless transmitter was implanted in Timmy's eyes. The transmitter sends the raw information from his eyes by $\mathrm{Wi}-\mathrm{Fi}$ to a receiver that was implanted in the auditory processing centres of Timmy's brain.)

5. (eye transmits to the visual processing unit): As part of an experiment, a wireless transmitter was implanted in Timmy's eyes. The transmitter sends the raw information from his eyes by Wi-Fi to a receiver that was implanted in the visual processing centres of Timmy's brain.)

6. (replace the conduit): As part of an experiment, the nerves connecting Timmy's eyes to the visual processing centres of his brain were replaced with artificial ones. The raw information from his eyes is now once again sent to the visual processing centres of Timmy's brain.

Timmy was then put in a room that was empty except for one blue box, one red box, and one green box (the boxes were identical in all respects except color). Timmy was instructed to put the blue box in front of the door. When the [smart phone/wireless transmitter/artificial nerves] [was/were] turned off, Timmy was unable to do this. When the [smart phone/transmitter/artificial nerves] [was/were] turned on, however, Timmy performed the task correctly and with no noticeable difficulty. 
Did Timmy see blue?

And the vignettes for Jimmy were as follows:

Jimmy (shown below) is a relatively simple robot built at a state university. Jimmy is

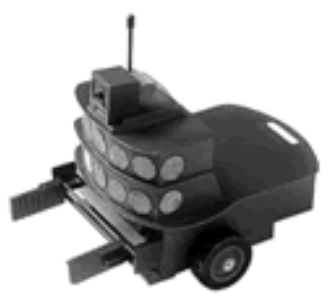
equipped with a microphone, a video camera, wheels for moving about, and two grasping arms for moving objects. The raw information from the microphone is sent to Jimmy's auditory processing unit, while the raw information from the video camera is sent to Jimmy's visual processing unit. Unfortunately, the conduit connecting the video camera to Jimmy's visual processing unit was damaged, so no information is sent to Jimmy's visual processing unit. Other than that, however, Jimmy was undamaged.

1. (phone processes and plays sounds): As part of an experiment, a smart phone is strapped to Jimmy's body. The phone processes the feed from its camera, detecting when there is an object in front of it and what color that object is. The phone then plays distinct sounds to transmit this information. The sounds are picked up by Jimmy's microphone.

2. (phone transmits raw feed to the auditory processing unit): As part of an experiment, a smart phone is strapped to Jimmy's body. The phone sends the raw feed from its camera by Wi-Fi to a receiver attached to Jimmy's auditory processing unit.

3. (phone transmits raw feed to the visual processing unit): As part of an experiment, a smart phone is strapped to Jimmy's body. The phone sends the raw feed from its camera by $\mathrm{Wi}-\mathrm{Fi}$ to a receiver attached to Jimmy's visual processing unit.)

4. (camera transmits to the auditory processing unit): As part of an experiment, a wireless transmitter was attached to Jimmy's video camera. The transmitter sends the raw information from the video camera by $\mathrm{Wi}-\mathrm{Fi}$ to a receiver attached to Jimmy's auditory processing unit. 
5. (phone transmits raw feed to the visual processing unit): As part of an experiment, a wireless transmitter was attached to Jimmy's video camera. The transmitter sends the raw information from the video camera by Wi-Fi to a receiver attached to Jimmy's visual processing unit.

6. (replace conduit): As part of an experiment, the conduit connecting the video camera to Jimmy's visual processing unit was replaced. The raw information from the video camera is now once again sent to Jimmy's visual processing unit.

Jimmy was then put in a room that was empty except for one blue box, one red box, and one green box (the boxes were identical in all respects except color). Jimmy was instructed to put the blue box in front of the door. When the [smart phone/wireless transmitter/video camera] was turned off, Jimmy was unable to do this. When the [smart phone/transmitter/video camera] was turned on, however, Jimmy performed the task correctly and with no noticeable difficulty.

Did Jimmy see blue?

Note that Scenario 1 and Scenario 2 for both agents satisfy neither condition (a) nor condition (b) described above. Scenario 3 satisfies (b) but not (a), while Scenario 4 satisfies (a) but not (b). Finally, Scenarios 5 and 6 satisfy both (a) and (b).

The study used the same recruitment method and restrictions as the previous studies, with the exception that for this study the ads were just run in North America. 317 responses were included in the analysis. ${ }^{57}$ The results are shown in Figure 5.

\footnotetext{
${ }^{57} 195$ eligible participants failed or did not answer the attention check. 87.6\% of participants (included in the analysis) indicated that they were native English-speakers, $71.9 \%$ women ( 3 non-binary), aged from 16 to 81 (average 41.3 years). ${ }^{57}$
} 

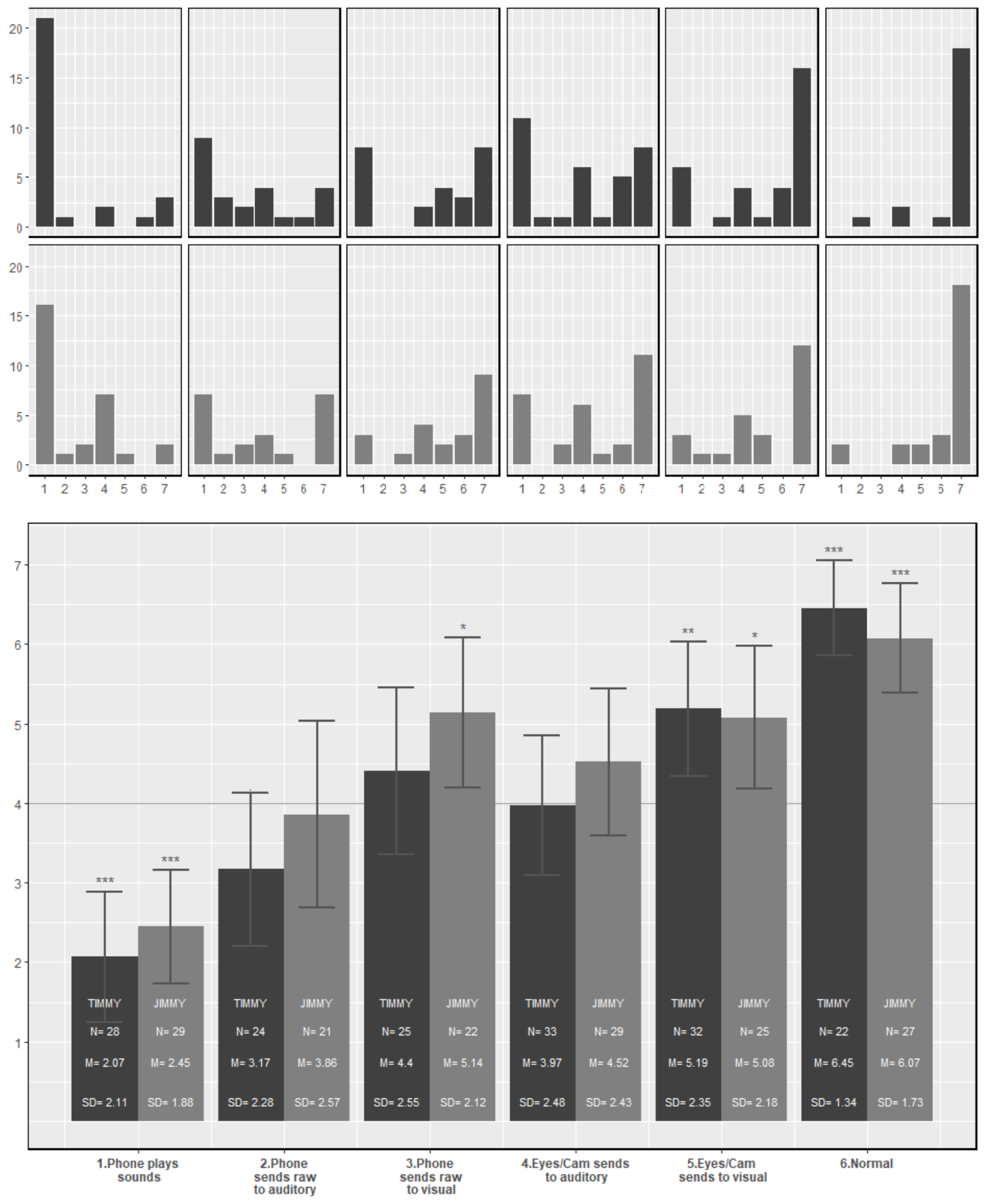
Figure 5: Mean responses for six vignettes in Ambiguity Study 4 with corresponding histograms above.

Let's first look at the results of (1) and (6) to see if the results of these cases replicate the previous studies. In (1), as in Ambiguity Study 1, people tended to deny that Timmy saw blue, and as in Ambiguity Study 2 and 3, the mean responses for both agents were below the neutral point. For Timmy and Jimmy, the mean response was below and significantly different from the neutral point. ${ }^{58}$ In (6), in line with SM and Ambiguity Study 1, the participants tended to affirm that Timmy and Jimmy saw blue with mean responses that were above and significantly different from the neutral point for each. ${ }^{59}$ In fact, the mean responses for the two agents weren't significantly different in either (1) or (6)..$^{60}$

More importantly for the purpose of this chapter is whether the results support the predictions of the Refined Ambiguity Objection. Remember that the first prediction of the objector was that conditions (a) and (b) being met (i.e. when the agent itself captures the visual information, and this information is processed by the visual processing units of the agent) should significantly increase the responses to the question of whether the agents see a given color. And the second prediction was that there should be a significant difference between Timmy's seeing and Jimmy's seeing even when these conditions are satisfied.

To see whether the results support the first prediction, I looked at cases (2)-(5) where three factors - the agent, the source of the information (input), and how the information is processed (output)—varied. A $2 \times 2 \times 2$ ANOVA showed a significant effect for how this information is

\footnotetext{
${ }^{58}$ Timmy: $M=2.07, N=28, S D=2.11, t(27)=-4.84, p<.001 ; 78.6 \%(N=22)$ answering 1,2 , or $3, \chi^{2}=9.14, d f=$ $1, p=0.0025$.

Jimmy: $M=2.45, N=29, S D=1.88, t(28)=-4.44, p<.001 ; 65.5 \%(N=19)$ answering 1,2 , or $3, \chi^{2}=2.79, d f=1$, $p=0.095$.

${ }^{59}$ Timmy: $M=6.45, N=22, S D=1.34, t(21)=8.62, p<.001 ; 86.4 \%(N=19)$ answering 5,6 , or $7, \chi^{2}=11.64, d f=$ $1, p<.001$.

Jimmy: $M=6.07, N=27, S D=1.73, t(26)=6.23, p<.001 ; 85.2 \%(N=23)$ answering 5,6 , or $7, \chi^{2}=13.37, d f=1$, $p<.001$.

${ }^{60}$ Timmy VS. Jimmy in (1): $t(53.82)=-0.71, p=0.48$, Cohen's $d=0.19$.

Timmy VS. Jimmy in (6): $t(48.69)=0.87, p=0.39$, Cohen's $d=0.24$.
}

100 
processed (output) by the agent while the effect for the source of information (input) was nearly significant. ${ }^{61}$ Considering that the output variable relates to conditions (b), this means that (b) is a significant factor. Further, Tukey post-hoc test indicated that (b) being met increased the responses to the question of whether the agents saw blue. ${ }^{62}$ That is, whether (b) is satisfied played a positive, significant role in shaping responses. This result supports the objector's prediction at least in the case (b). However, as noted, for the objection to be confirmed, the agent factor should also be significant. Unfortunately for the objector, the results of the same ANOVA test indicated that there is no significant main or interaction effect for the agent variable. ${ }^{63}$ In line with this, no significant difference was found between Timmy and Jimmy across (2)-(5). ${ }^{64}$ So, against the Refined Ambiguity Objection, there is no reason to think that people are interpreting "see" one way for the robot and another way for Timmy.

Considering the results of this study together with SM, (Sytsma, 2014) and the previous Ambiguity Studies (1, 2 and 3), we have a good reason to think that laypeople apply roughly the same concept of "seeing colors" for both the simple robot and the human being. After all, in the standard conditions, people tend to ascribe seeing the target color both to the robot and the human while they tend to deny ascribing seeing the target color to both agents in the unusual cases of Ambiguity Studies 2-3 (in which the agent gets the information about the location of the target object through a sound produced by a mobile phone). So, lay ascriptions of seeing do not vary notably between the agents in these cases. And the same is also true for the Wi-Fi cases in the present study: there was no significant difference between Timmy and Jimmy for any of these cases. So, if people

\footnotetext{
${ }^{61}$ OUTPUT: $F(1,203)=10.66, p=0.0012$.

INPUT: $F(1,203)=2.66, p=0.10$.

${ }^{62}$ For output variable (whether visual information is processed by auditory or visual processing units), the difference in means between was 1.069 in favour of processing by visual units.

${ }^{63}$ AGENT: $F(1,203)=1.66, p=0.20$.

AGENT: INPUT: $F(1,203)=0.51, p=0.48$.

AGENT: OUTPUT: $F(1,203)=0.27, p=0.6$. AGENT: INPUT: OUTPUT: $F(1,203)=0.28, p=0.6$.

${ }^{64}(2): t(40.33)=-0.95, p=0.35$, Cohen's $d=0.29$.

(3): $t(44.88)=-1.08, p=0.29$, Cohen's $d=0.31$.

(4): $t(59.28)=-0.88, p=0.38$, Cohen's $d=0.22$.

(5): $t(55.33)=0.18, p=0.86$, Cohen's $d=0.047$.
} 
endorse that Jimmy can see colors because they treat seeing colors for robots in the informational sense, then there is a good reason to think that they do the same for humans, which clearly contradicts the core idea behind the Ambiguity Objection-that laypeople read seeing colors differently for a simple robot and for a human.

\subsection{Concluding Remarks}

To further assess the Ambiguity Objection, the present chapter discussed four new experiments testing two main predictions that naturally follow from the objection. The first prediction was that since people read "seeing red" in the informational sense for robots, if the participants were given more "phenomenal" options such as "experiencing colors", they would deny that a simple robot could experience colors even if they would endorse that it could see colors. Ambiguity Study 1 indicates against this, showing that people tend to endorse both experiencing and seeing colors for the simple robot. The second prediction was that laypeople would be happy to ascribe seeing colors to a simple robot as long as it captures and processes the relevant information. The results of Ambiguity Study 2, however, indicate against this prediction. Ambiguity Studies 3 and 4 tested the predictions of possible objections to Ambiguity Study 2 and the results indicated against these predictions as well. So, these studies collectively cast further doubt on the Ambiguity Objection and thereby offer additional empirical support to SM's conclusion that common aspect intuitions are not prevalent and thus that Premise 2-1 holds.

It is also worth underlining that the studies discussed in this chapter do not only suggest against the Ambiguity Objection but also provide further empirical support for Premise 2-1: Ambiguity Study 1 successfully replicates all the main findings of SM while Ambiguity Studies 3 and 4 further show that laypeople tend to endorse that a simple robot can see colors, which does not accord well with the standard conception of phenomenal consciousness.

This and the last chapter make a strong case against the common aspect claim - the claim that laypeople share common aspect intuitions. However, there is another kind of experimental study that is relevant to this claim; namely, studies on the dimensions of the folk conception of mind. 
The next chapter will discuss the existing studies on this issue and introduce new ones to further investigate the prevalence of common aspect intuitions. 


\section{A GAP BETWEEN PERCEPTUAL STATES AND OTHER "PHENOMENAL" STATES IN THE FOLK CONCEPTION OF MIND}

The previous two chapters provided support for Premise 2-1-that existence intuitions (experiential property and common aspect intuitions) are not widespread-by discussing the results of existing and new empirical studies. This chapter aims to further establish Premise 2-1 by detailing studies concerning the dimensions of the folk conception of mind. As will be explained below, these studies provide additional empirical data that help us to assess the prevalence of common aspect intuitions and hence the plausibility of Premise 2-1.

Remember that SM's challenge to the common aspect claim-the claim that common aspect intuitions are widespread - was that laypeople seem to treat two prototypical cases of so-called phenomenal states differently. The studies on the dimensions of the lay conception of mind provide us with a new perspective to assess if laypeople tend to classify so-called phenomenal states similarly. These studies typically ask the participants about various mental capabilities or states and investigate which mental states/capabilities were given similar answers and which dimensions (clusters) they form. To give a toy example, imagine that the following six questions were asked to participants in a study: Could robots see colors/feel pain/enjoy music/detect colors/detect damage/distinguish one song from another? Note that the first three items refer to so-called phenomenal states while the other three do not. If laypeople recognize the phenomenality in the first three items, then we would expect them to tend to give negative answers for these items (on the plausible assumption that they consider robots as non-experiencers) and tend to give positive answers for the last three non-phenomenal items. In this case, factor/cluster analysis tools (such as exploratory factors analysis, principal component analysis and cluster analysis) would suggest that there are two clusters, one being comprised of the three phenomenal states and the other being comprised of the three non-phenomenal states (detecting colors/detecting damage/distinguishing one song from another). In such a case, it would be plausible to assume that what bundles seeing colors/feeling pain/enjoying music together is a common factor, namely what-it-is-likeness, that is shared by these states. Following this logic, the studies on the lay conception of mind ask participants about an extensive range of mental states and capabilities and look at the possible 
dimensions comprised of mental capabilities bundled by a common factor. For example, feeling disrespected/embarrassed/excluded might be the items of the same dimension because of the social aspect they involve.

As hinted above, if laypeople recognize phenomenality in various mental states and capabilities, we would expect that the studies on the lay conception of mind would suggest that perceptual states and other so-called phenomenal states would be in the same dimension because of the common aspect they share. In other words, we would expect laypeople to classify these states similarly, forming a dimension, and to classify other (non-phenomenal) states similarly, forming another dimension. If participants' classification indicated two dimensions that mirror the philosophical distinction between phenomenal states and non-phenomenal states, this would be suggestive of the common aspect claim. On the other hand, if the phenomenal states fell across different dimensions, this would suggest that laypeople tend to treat these states dissimilarly, which, as in SM, casts doubt on the common aspect claim, which in turn would further support Premise 2-1.

Motivated by this line of reasoning, this chapter will first discuss three studies on how laypeople conceptualize the mind: Gray, Gray, and Wegner (2007) in §6.1; Weisman, Dweck, and Markman (2017) in §6.2; and Malle (2019) in §6.3. §6.4 presents a new study (Dimension Study 1). I argue that Weisman et al. (2017), Malle (2019) and Dimension Study 1 together indicate that laypeople classify so-called phenomenal states into different dimensions, with various examples of these states falling across different dimensions. However, one can object to my interpretation of these studies by claiming that the perceptual states asked about in these studies are ambiguous and do not uniformly trigger a phenomenal reading. To test this objection, $\S 6.5$ presents the results of a further experiment (Dimension Study 2) that includes some questions about more clearly "phenomenal" mental states (such as seeing colors and experiencing sounds). As will be seen, this study also suggests a gap between perceptual states and other so-called phenomenal states in the lay conception of mind, which suggests that they do not tend to recognize the supposed "phenomenal" commonality between these states and thus that they do not tend to share common aspect intuitions. Finally, in $§ 6.6$, I will reply to a general objection that challenges the above- 
mentioned reasoning. I conclude that the studies discussed in this chapter provide us with additional empirical evidence against the common aspect claim and in favour of Premise 2-1 by revealing a gap between perceptual and non-perceptual states in the folk conception of mind.

\subsection{Gray, Gray and Wegner's Study}

Gray and her colleagues investigated the dimensions of lay perception of mind by asking participants to compare pairs of different characters in terms of either their cognitive capacities or the participant's affection towards the characters. In the study, each participant was asked to compare different agents' capabilities for a particular cognitive capacity. To give an example, some participants were asked to rate whether a 5-year-old is more or less likely to be able to feel pain than is a chimpanzee (Gray et al., 2007, p. 619). 2040 unique respondents completed 2399 surveys that called for 78 pairwise comparisons of 13 characters (including seven living human forms, three non-human animals, a dead woman, God and a sociable robot) for one of 18 mental capacities (such as hunger, fear, pain, morality, communication, thought, joy, self-control) or for one of six personal judgements (such as “which character do you like more?”).

The authors found that some mental capacities (such as hunger, fear, pain, pleasure, rage) are correlated with each other and thus form a dimension that they labelled "Experience" while some others (such as self-control, morality and memory) form a second dimension they called "Agency". Figure 6 shows the locations of the agents in the two-dimensional mental space. 


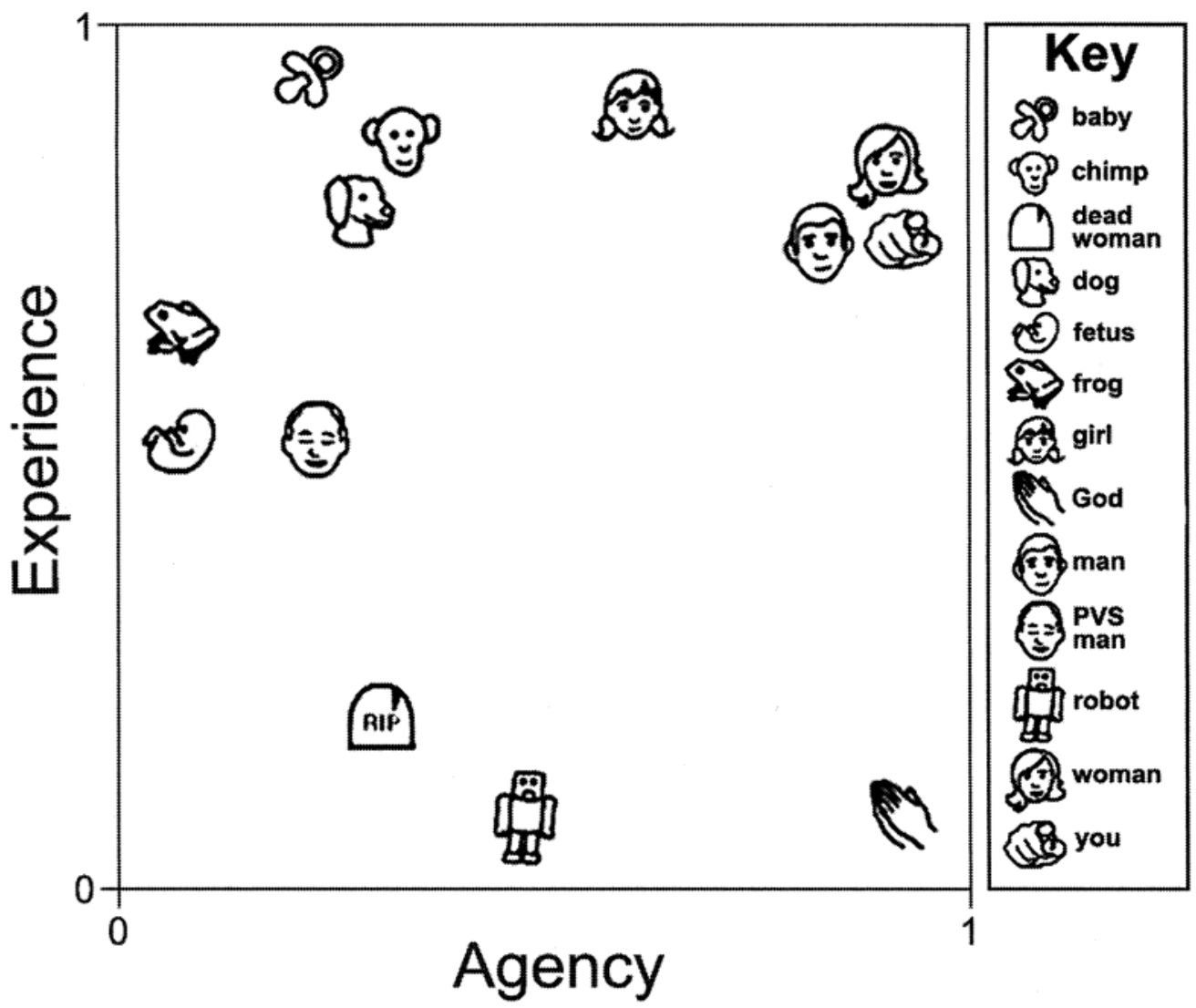

Figure 6: 13 agents located in the two-dimensional mental coordinate system in Gray et al. (2007).

As can be seen from Figure 6, frog and foetus got low scores on Agency but average scores on Experience while adult humans received high scores on both dimensions. God and the robot were treated as being much higher in Agency than Experience.

The key question this study raises is the relation between the dimension of Experience and phenomenality. Taking the Experience dimension to coincide with phenomenality, the study provides prima facie support for the common aspect claim insofar as it suggests that people tend to treat living organisms as being high in Experience whereas they see robots as being low in Experience. And, in fact, all of the phenomenal states asked about in the study fell under the Experience dimension. Further, ratings for the entities on this dimension plausibly cohere with the standard, philosophical conception of phenomenality. 
However, despite the authors' choice of labelling, the Experience dimension does not capture the philosophical sense of subjective experience. First, the dimension includes the items "consciousness" and "personality". The term "consciousness" is arguably too broad to be assumed to indicate phenomenal consciousness, which is a highly specific notion of consciousness. And the capability of having a personality would not standardly be taken to be a phenomenal mental capability. Second, and more importantly, as pointed out in SM, the experience cluster includes only non-perceptual cases. However, perceptual cases are considered prototypical examples of phenomenal states. As such, the study does not give us any indication with regard to whether laypeople tend to treat perceptual states and other so-called phenomenal states similarly or not, and thus does not actually indicate whether what bundles the items in the Experience dimension is the phenomenality of these items or not. As will be seen in the next three sections, subsequent studies involving questions about perceptual states suggest that the folk conception of mind has three rather than two dimensions, although there are some differences in how these dimensions are construed, with perceptual states and non-perceptual "phenomenal" states tending to fall across different dimensions. I argue that these findings cast further doubt on the common aspect claim.

\subsection{Weisman, Dweck, and Markman's Study}

As noted, the two-dimensional lay conception of mind suggested by Gray et al. (2007) can be read as suggestive of a folk conception of phenomenality in that the participants seem to distinguish between the agents in terms of their capacity for experience. It was also noted, however, that the mental capabilities tested that fell under the Experience dimension did not include perceptual states, which are regarded as paradigm cases of phenomenal consciousness. So, it would not be plausible to simply equate the Experience dimension with phenomenality. This is because, if participants were also asked about perceptual cases, another dimension might emerge that includes perceptual capabilities but not the other so-called phenomenal items that fell under the Experience dimension. Indeed, Weisman et al. (2017) included perceptual items and their findings suggest that the folk conception of mind involves three dimensions with some perceptual states and bodily sensations falling across different dimensions. The main motivation behind the work of Kara

Weisman and her colleagues was that the Experience-Agency framework was insensitive to some 
well-established distinctions between perception, emotion and desire, which fall under the same Experience dimension in Gray et al. (2007) (Weisman et al., 2017, p. 11374). So, they doubted that Gray and her colleagues' framework successfully captured the lay intuitions about the structure of mental life. The main reason for this, they plausibly claim, was that each participant compared pairs of agents' capability for only one mental capacity, which led them to focus on the similarities and differences between the agents rather than the mental capacities. So, the similarities between agents' ranks for two different mental capacities in the overall does not necessarily mean that the participants treated those capacities similarly (p. 11374). To assess better which mental capacities are treated similarly by people, Weisman and her colleagues took the alternative way of asking each participant about different mental capacities for only one agent (pp. 11374-11375).

Weisman and her colleagues designed four experiments in which the participants were asked to evaluate 40 mental capacities for a given agent. These mental capabilities included agentic (such as having intentions, self-awareness, working toward a goal), physiological (getting hungry, experiencing pain, feeling tired, and so on), emotional (such as experiencing fear and pleasure), cognitive (holding beliefs, doing computations, etc.), perceptual (seeing things, perceiving depth, and so on), and social capabilities (such as recognizing someone, experiencing guilt). In Studies 1-3, there were only two agents (a beetle and a robot), while Study 4 involved 21 different entities (such as an adult, an infant, a person in a persistent vegetative state, chimpanzee, frog, a robot, and a car). Each participant was asked to rate the extent to which a single one of these entities is capable of having 40 mental capacities.

Independent exploratory factor analyses for each of the four studies revealed the same structure with three dimensions. The first dimension is what the authors labelled as Body and covers “abilities related to the physical, biological body" (p. 11375). This dimension includes mostly bodily sensations such as getting hungry, feeling safe, tired, nauseated and calm, experiencing pain, fear, and pleasure. The second dimension, Heart, corresponds primarily to basic and social emotions as well as social-cognitive and self-regulatory abilities that "resonate with the metaphorical sense of heart and perhaps with some notions of 'spirit' or 'soul'" (p. 11375). The 
Heart dimension was dominant for feeling embarrassed, depressed, disrespected, experiencing pride and guilt, holding beliefs, and understanding the feelings of others, telling right from wrong, and exercising self-restraint. Lastly, the Mind dimension covers perceptual and cognitive abilities and was the dominant factor for seeing and remembering things, perceiving depth, sensing temperatures, detecting sounds, recognizing, and communicating with others.

This three-dimensional structure is suggestive of the idea that the folk treat perceptual states and bodily sensations differently. As noted, this is in tension with the common aspect claim in that if participants recognize the common aspect of phenomenality in different so-called phenomenal states, we would expect them to tend to classify "phenomenal states" into the same dimension. In contrast to this expectation, and in line with SM and follow-up studies, participants tended to classify the perceptual states (perceiving depth, detecting odours, seeing things and sensing temperatures) and other so-called phenomenal states (bodily sensations, emotions and moods such as experiencing pain, feeling nauseated, feeling tired, experiencing fear, experiencing pleasure) into different dimensions. It is also noteworthy that, again in line with SM and follow-up studies, participants endorsed that a robot has perceptual capabilities such as sensing temperatures, seeing things and perceiving depth while they were reluctant to ascribe the other items falling under the Body dimension which mostly covers bodily sensations, emotions and moods as well as others subsumed under the Heart dimension. The key finding that laypeople treat perceptual states and other so-called phenomenal states differently in this study is confirmed by another study on the lay conception of mind, namely Malle (2019), which I will discuss in the next section.

\subsection{Malle's Study}

Malle (2019) investigated how laypeople conceptualize mental capacities across four studies using varied and large item pools including capacities of perception, cognition, emotion, agency, learning, social cognition, social-moral capacities, and communication. In Study 1, each participant was asked about one of 16 agents (e.g., human adult, robot, rabbit, chimpanzee, as well as group agents such as a city council and a large company). They were asked to rate the extent to which the agent is capable of 28 mental capacities such as hunger, pain, joy, believing, emotion, 
desire, self-control, reasoning, see or hear, taste or smell). The second study added 30 new mental capacities to the item pool and reduced the variety in agents. ${ }^{65}$ Malle's third and fourth studies asked the participants to rate the mental capacities they would want or not in an advanced home robot rather than to rate if the robot is capable of having those capacities. As such, it would be controversial to discuss the result of these studies to assess the common aspect claim. So, I will focus on only the first and second studies.

The results of the first and second studies suggested the same (three) dimensions each of which includes consistently classified items. As Malle pointed out, these dimensions cohere with Weisman et al. (2017) and Kozak, Marsh, and Wegner (2006) (p. 2273). ${ }^{66}$ The first dimension, which Malle terms "Affect", is similar to the Experience dimension of Gray et al. (2007) and the Body dimension of Weisman et al. (2017) in that it covers bodily sensations, emotions, moods and some physiological states such as feeling pain/tired/happy/stress/pleasure, experiencing fear and gratitude and so on. As in Weisman et al. (2017), the Agency dimension of Gray et al. (2007) was divided into two dimensions by Malle — what he termed "Moral and Mental Regulation" and "Reality Interaction". The Moral and Mental Regulation dimension covers mostly intentional states (such as believing certain things, planning, and deliberating) and moral items (feel shame, pride, having values and so on). Finally, the Reality Interaction dimension, like the Mind dimension in Weisman et al. (2017), covers the items related to perception, cognition and communication such as seeing and hearing the world, sensing temperature, perceiving things, remembering past events, making decisions, having accurate knowledge, communicating verbally and so on.

\footnotetext{
${ }^{65}$ Each participant in the second study was asked about one of the following agents: average adult, a two-year-old child, a cat, and a home care robot.

${ }^{66}$ Kozak and her colleagues concluded in this study that the lay conception of mind has three dimensions. Emotion dimension is similar to Experience dimension in Gray et al. (2007) and covers feelings, pain, emotion, pleasure. Intention dimension is similar to the Agency dimension and subsumes planning, goals, and purposeful actions. Lastly, Cognition dimension is similar to Mind dimension of Weisman et al. (2017) and consists of consciousness, memory and thought.
} 
Focusing on whether the participants in Malle's first and second studies classified perceptual states and other supposedly phenomenal states similarly or not, we find that seeing or hearing things, perceiving things/having perceptions and feeling/sensing temperature/touch primarily fell under the Reality Interaction dimension while the other so-called phenomenal states such as feeling pain, experiencing pleasure, feeling tired consistently fell under the dimension of Affect. In brief, like Weisman et al. (2017), Malle`s study suggests that laypeople classify perceptual states and other allegedly phenomenal states differently. As will be seen in the next two sections, this finding is confirmed by two new studies.

\subsection{Dimension Study 1}

As a part of a larger project that concerns the lay judgements about the mental states/capabilities of sophisticated robots using different types of robot agents, Dimension Study 1 is a cross-cultural study that asked participants whether a robot that ran a simulation of the participant's brain was capable of the capacities tested by Weisman and her colleagues. For the current purpose of the chapter, I will discuss the study in terms of the possible gap between perceptual and non-perceptual

phenomenal states in the folk conception of mind. ${ }^{67}$ Accordingly, the dimensions the study suggests will be discussed for the limited purpose of seeing whether there is such a gap or not. So, I will first look at whether participants tended to give similar answers for perceptual and nonperceptual phenomenal states and then look at the dimensions the study suggests in order to see whether perceptual and non-perceptual phenomenal states fall under the same or different dimensions.

The study used the same global recruitment strategy, restrictions, and basic set-up as in the previous studies. Participants were given the following probe:

${ }^{67}$ By using a sophisticated robot as the agent, the study aimed to expand upon the simple robot used in Weisman et al. (2017) and Malle (2019) to see if there would still be a gap between perceptual states and other so-called phenomenal states in the folk conception of mind when such a more human-like robot is used. 
Imagine that in the future scientists are able to exactly scan a person's brain at the molecular level. Using the information from the brain scan they can create a perfect computer simulation of the working brain. They can then embed that computer in a robot body to create a robot version of the person.

Imagine that scientists scan your brain and use that information to create a robot version of you. On a scale of 0 (Not at all capable) to 6 (Highly capable), how capable would the robot be of...

They were then asked about the same 40 capacities tested by Weisman et al. (2017), as well as the attention check they used ("please select 4 for this item"). The resulting 41 questions were presented in random order. Participants missing the attention check were excluded from the analysis, leaving 348 responses. ${ }^{68}$

40 one-sample t-tests were conducted using the Holm-Bonferroni correction to compare the mean response for each capacity to the neutral point. Participants tended to ascribe all of the mental capabilities to the robot, except for feeling nauseated $(M=2.95)$. The mean ratings for the remaining 39 items were all above and significantly different from the neutral point, with having thoughts having the greatest mean (5.03) and feeling disrespected having the lowest mean (3.55). ${ }^{69}$

68184 eligible participants failed or did not answer the attention check. Participants (included in the analysis) were $47.9 \%$ native English-speakers, $69.5 \%$ women (two non-binary), aged from 16 to 86 (average 25.9 years).

${ }^{69}$ happy: $M=4.02, N=348, S D=1.79, t(347)=10.63, p<.001 ; 65.2 \%(N=227)$ answering 4,5, or $6, \chi^{2}=32.29$, $d f=1, p<.001$.

depressed: $M=3.57, N=348, S D=2.05, t(347)=5.24, p<.001 ; 55.5 \%(N=193)$ answering 4,5 , or $6, \chi^{2}=4.15$, $d f=1, p=0.042$.

fear: $M=3.77, N=348, S D=1.93, t(347)=7.42, p<.001 ; 60.3 \%(N=210)$ answering 4,5, or $6, \chi^{2}=14.9, d f=1$, $p<.001$.

angry: $M=3.82, N=348, S D=1.92, t(347)=7.97, p<.001 ; 60.6 \%(N=211)$ answering 4,5, or $6, \chi^{2}=15.74, d f=$ $1, p<.001$.

calm: $M=3.81, N=348, S D=1.79, t(347)=8.47, p<.001 ; 60.6 \%(N=211)$ answering 4,5, or $6, \chi^{2}=15.74, d f=$ $1, p<.001$.

sounds: $M=4.67, N=348, S D=1.44, t(347)=21.65, p<.001 ; 79.3 \%(N=276)$ answering 4,5, or $6, \chi^{2}=119.59$, $d f=1, p<.001$. 
seeing: $M=4.39, N=348, S D=1.81, t(347)=14.35, p<.001 ; 72.7 \%(N=253)$ answering 4,5, or $6, \chi^{2}=71.74, d f$ $=1, p<.001$.

temperature: $M=4.45, N=348, S D=1.54, t(347)=17.46, p<.001 ; 73.9 \%(N=257)$ answering 4,5 , or $6, \chi^{2}=$ 79.18, $d f=1, p<.001$.

odours: $M=4.40, N=348, S D=1.59, t(347)=16.4, p<.001 ; 73.9 \%(N=257)$ answering 4,5, or $6, \chi^{2}=79.18, d f$ $=1, p<.001$.

depth: $M=4.11, N=348, S D=1.59, t(347)=13.07, p<.001 ; 66.1 \%(N=230)$ answering 4,5, or $6, \chi^{2}=36.05, d f$ $=1, p<.001$.

computations: $M=3.71, N=348, S D=1.65, t(347)=8.05, p<.001 ; 54 \%(N=188)$ answering 4,5, or $6, \chi^{2}=2.25$, $d f=1, p=0.13$.

thoughts: $M=5.03, N=348, S D=1.4, t(347)=26.94, p<.001 ; 87.4 \%(N=304)$ answering 4,5, or $6, \chi^{2}=194.25$, $d f=1, p<.001$.

reasoning: $M=4.47, N=348, S D=1.43, t(347)=19.23, p<.001 ; 79 \%(N=275)$ answering 4,5 , or $6, \chi^{2}=117.25$, $d f=1, p<.001$.

remembering: $M=4.42, N=348, S D=1.49, t(347)=17.78, p<.001 ; 76.4 \%(N=266)$ answering 4,5 , or $6, \chi^{2}=$ 97.29, $d f=1, p<.001$.

beliefs: $M=3.99, N=348, S D=1.83, t(347)=10.06, p<.001 ; 64.7 \%(N=225)$ answering 4,5, or $6, \chi^{2}=29.9, d f$ $=1, p<.001$.

hungry: $M=4.09, N=348, S D=1.91, t(347)=10.63, p<.001 ; 66.7 \%(N=232)$ answering 4,5, or $6, \chi^{2}=38.67, d f$ $=1, p<.001$.

tired: $M=4.20, N=348, S D=1.76, t(347)=12.71, p<.001 ; 71.8 \%(N=250)$ answering 4,5, or $6, \chi^{2}=66.39, d f=$ $1, p<.001$.

pain: $M=4.05, N=348, S D=1.92, t(347)=10.18, p<.001 ; 67.2 \%(N=234)$ answering 4,5, or $6, \chi^{2}=41.38, d f=$ $1, p<.001$.

nauseated: $M=2.95, N=348, S D=1.85, t(347)=-0.46, p=0.64 ; 38.2 \%(N=133)$ answering 0,1, or $2, \chi^{2}=19.32$, $d f=1, p<.001$.

safe: $M=3.87, N=348, S D=1.71, t(347)=9.48, p<.001 ; 62.1 \%(N=216)$ answering 4,5, or $6, \chi^{2}=20.28, d f=$ $1, p<.001$.

love: $M=4.11, N=348, S D=1.9, t(347)=10.89, p<.001 ; 68.1 \%(N=237)$ answering 4,5, or $6, \chi^{2}=45.62, d f=$ $1, p<.001$.

recognizing: $M=4.73, N=348, S D=1.49, t(347)=21.6, p<.001 ; 82.2 \%(N=286)$ answering 4,5, or $6, \chi^{2}=144.18$, $d f=1, p<.001$.

communicating: $M=4.36, N=348, S D=1.56, t(347)=16.23, p<.001 ; 73 \%(N=254)$ answering 4,5 , or $6, \chi^{2}=$ 73.56, $d f=1, p<.001$.

guilt: $M=3.93, N=348, S D=1.92, t(347)=9.07, p<.001 ; 64.7 \%(N=225)$ answering 4,5, or $6, \chi^{2}=29.9, d f=1$, $p<.001$.

disrespected: $M=3.55, N=348, S D=1.96, t(347)=5.2, p<.001 ; 53.2 \%(N=185)$ answering 4,5, or $6, \chi^{2}=1.39$, $d f=1, p=0.24$.

free will: $M=4.37, N=348, S D=1.61, t(347)=15.84, p<.001 ; 74.1 \%(N=258)$ answering 4,5, or $6, \chi^{2}=81.1, d f$ $=1, p<.001$.

choices: $M=4.32, N=348, S D=1.57, t(347)=15.67, p<.001 ; 73 \%(N=254)$ answering 4,5, or $6, \chi^{2}=73.56, d f$ $=1, p<.001$.

self-restraint: $M=3.8, N=348, S D=1.65, t(347)=9.09, p<.001 ; 61.8 \%(N=215)$ answering 4,5, or $6, \chi^{2}=19.32$, $d f=1, p<.001$.

intentions: $M=4.29, N=348, S D=1.57, t(347)=15.26, p<.001 ; 73 \%(N=254)$ answering 4,5 , or $6, \chi^{2}=73.56$, $d f=1, p<.001$. 
As such, there is no difference in participants' judgements between phenomenal and nonphenomenal cases because the participants gave positive answers to all supposedly phenomenal cases (such as experiencing pain, experiencing joy, feeling happy, sensing temperatures) and nonphenomenal cases (such as telling right from wrong, reasoning about things and making choices). ${ }^{70}$

goal: $M=4.42, N=348, S D=1.58, t(347)=16.72, p<.001 ; 73.3 \%(N=255)$ answering 4,5, or $6, \chi^{2}=75.41, d f=$ $1, p<.001$.

conscious: $M=4.51, N=348, S D=1.5, t(347)=18.77, p<.001 ; 77 \%(N=268)$ answering 4,5, or $6, \chi^{2}=101.56$, $d f=1, p<.001$.

self-aware: $M=4.46, N=348, S D=1.59, t(347)=17.12, p<.001 ; 75.9 \%(N=264)$ answering 4,5, or $6, \chi^{2}=93.1$, $d f=1, p<.001$.

desires: $M=4.51, N=348, S D=1.73, t(347)=16.28, p<.001 ; 78.4 \%(N=273)$ answering 4,5, or $6, \chi^{2}=112.66$, $d f=1, p<.001$.

embarrassed: $M=3.78, N=348, S D=1.91, t(347)=7.63, p<.001 ; 57.8 \%(N=201)$ answering 4,5, or $6, \chi^{2}=8.38$, $d f=1, p=0.0038$.

emotional recognition: $M=4.64, N=348, S D=1.5, t(347)=20.34, p<.001 ; 79.9 \%(N=278)$ answering 4, 5, or 6, $\chi^{2}=124.32, d f=1, p<.001$.

joy: $M=4.19, N=348, S D=1.78, t(347)=12.5, p<.001 ; 70.4 \%(N=245)$ answering 4,5, or $6, \chi^{2}=57.94, d f=1$, $p<.001$.

morality: $M=4.65, N=348, S D=1.56, t(347)=19.67, p<.001 ; 78.4 \%(N=273)$ answering 4,5, or $6, \chi^{2}=112.66$, $d f=1, p<.001$.

personality: $M=4.56, N=348, S D=1.64, t(347)=17.71, p<.001 ; 77.6 \%(N=270)$ answering 4,5, or $6, \chi^{2}=$ 105.93, $d f=1, p<.001$.

pleasure: $M=4.02, N=348, S D=1.76, t(347)=10.83, p<.001 ; 65.5 \%(N=228)$ answering 4,5 , or $6, \chi^{2}=33.52$, $d f=1, p<.001$.

pride: $M=3.72, N=348, S D=1.81, t(347)=7.37, p<.001 ; 55.2 \%(N=192)$ answering 4,5, or $6, \chi^{2}=3.72, d f=$ $1, p=0.054$.

${ }^{70}$ We found that there are significant, positive, but negligible correlations between "angry", "intentions" and "pride" for the CRT 2 scores (but not CRT), and between "computations" and the CRT scores (but not CRT 2). So, we can safely claim that cognitive reflection score was not a significant factor for participants' responses to philosophical questions:

happy, CRT: $r=-0.07, p=0.27, N=219$; CRT 2: $r=-0.07, p=0.31, N=238$.

depressed, CRT: $r=-0.05, p=0.44, N=219$; CRT 2: $r=0.03, p=0.65, N=238$.

fear, CRT: $r=-0.13, p=0.052, N=219$; CRT 2: $r=0.046, p=0.48, N=238$.

angry, CRT: $r=-0.01, p=0.89, N=219$; CRT 2: $r=0.14, p=0.037, N=238$.

calm, CRT: $r=0.038, p=0.57, N=219$; CRT 2: $r=0.049, p=0.45, N=238$.

sounds, CRT: $r=0.05, p=0.46, N=219$; CRT 2: $r=-0.03, p=0.66, N=238$.

seeing, CRT: $r=0, p=0.96, N=219$; CRT 2: $r=0.029, p=0.65, N=238$.

temperature, CRT: $r=0.0079, p=0.91, N=219$; CRT 2: $r=-0.05, p=0.41, N=238$.

odours, CRT: $r=-0.04, p=0.6, N=219$; CRT 2: $r=0.023, p=0.72, N=238$.

depth, CRT: $r=0.11, p=0.1, N=219$; CRT 2: $r=0.1, p=0.11, N=238$.

computations, CRT: $r=0.18, p=0.0092, N=219$; CRT 2: $r=0.041, p=0.53, N=238$.

thoughts, CRT: $r=0.04, p=0.56, N=219$; CRT 2: $r=0.094, p=0.15, N=238$. 
To investigate the dimensions of mind perception suggested by the results of this study, I first conducted a parallel analysis to determine the factor count using the minimum residual factor algorithm. Parallel analysis with minimum residuals solution suggested that the 40 capabilities can be reduced into four factors. The analysis with maximum likelihood, weighted least squares, and generalized weighted least-squares solutions also suggested the same factor count. ${ }^{71}$ I then conducted an exploratory factor analysis with four factors with the minimum residual factor algorithm and oblimin rotation. ${ }^{72}$ I retained all factors as each accounted for at least $5.0 \%$ of the variance. To determine which items fall under which factors, following the suggestion of Pituch and Stevens (2015, p. 346), I applied the threshold of |.40| to the factor loadings. And to determine which items should be assigned to a factor, I applied the following rule: the item should have a

reasoning, CRT: $r=0.056, p=0.41, N=219$; CRT 2: $r=0.029, p=0.66, N=238$.

remembering, CRT: $r=-0.09, p=0.16, N=219$; CRT 2: $r=-0.03, p=0.61, N=238$.

beliefs, CRT: $r=-0.06, p=0.34, N=219$; CRT 2: $r=0.018, p=0.78, N=238$. hungry, CRT: $r=-0.02, p=0.79, N=219$; CRT 2: $r=0.075, p=0.25, N=238$. tired, CRT: $r=-0.05, p=0.5, N=219$; CRT 2: $r=0.094, p=0.15, N=238$. pain, CRT: $r=-0.09, p=0.19, N=219$; CRT $2: r=0.0036, p=0.96, N=238$. nauseated, CRT: $r=0.0042, p=0.95, N=219$; CRT 2: $r=0.05, p=0.44, N=238$. safe, CRT: $r=-0.05, p=0.45, N=219$; CRT 2: $r=-0.01, p=0.88, N=238$. love, CRT: $r=0.014, p=0.83, N=219$; CRT 2: $r=0.0078, p=0.9, N=238$. recognizing, CRT: $r=-0.04, p=0.56, N=219$; CRT 2: $r=-0.06, p=0.32, N=238$. communicating, CRT: $r=-0.01, p=0.85, N=219$; CRT 2: $r=0.034, p=0.6, N=238$. guilt, CRT: $r=-0.04, p=0.52, N=219$; CRT 2: $r=0.09, p=0.17, N=238$. disrespected, CRT: $r=-0.04, p=0.54, N=219$; CRT 2: $r=0.067, p=0.31, N=238$. free will, CRT: $r=0.0026, p=0.97, N=219$; CRT 2: $r=0.11, p=0.079, N=238$. choices, CRT: $r=-0.13, p=0.057, N=219$; CRT 2: $r=0.001, p=0.99, N=238$. self-restraint, CRT: $r=-0.03, p=0.63, N=219$; CRT 2: $r=-0.07, p=0.27, N=238$. intentions, CRT: $r=0.023, p=0.73, N=219$; CRT 2: $r=0.17, p=0.0068, N=238$. goal, CRT: $r=0.039, p=0.57, N=219$; CRT 2: $r=0.055, p=0.4, N=238$. conscious, CRT: $r=-0.01, p=0.88, N=219$; CRT $2: r=0.022, p=0.73, N=238$. self-aware, CRT: $r=0.041, p=0.54, N=219$; CRT 2: $r=-0.02, p=0.78, N=238$. desires, CRT: $r=0.036, p=0.6, N=219$; CRT 2: $r=-0.01, p=0.91, N=238$. embarrassed, CRT: $r=0, p=0.95, N=219$; CRT 2: $r=0.078, p=0.23, N=238$. emotional recognition, CRT: $r=0.014, p=0.84, N=219$; CRT 2: $r=-0.04, p=0.58, N=238$. joy, CRT: $r=-0.09, p=0.18, N=219$; CRT 2: $r=-0.01, p=0.87, N=238$. morality, CRT: $r=-0.01, p=0.88, N=219$; CRT 2: $r=0.058, p=0.37, N=238$. personality, CRT: $r=0.027, p=0.69, N=219$; CRT 2: $r=-0.01, p=0.84, N=238$. pleasure, CRT: $r=0.033, p=0.62, N=219$; CRT 2: $r=0.016, p=0.8, N=238$. pride, CRT: $r=0.049, p=0.48, N=219$; CRT 2: $r=0.14, p=0.034, N=238$.

71 The scree plot of principal component analysis suggested 3 or 4 factors would be sufficient.

${ }^{72}$ Factor analysis with minimum residual solution and different rotation methods such as varimax and promax resulted in similar dimensions. The analysis with maximum likelihood solution and oblimin rotation also indicated the similar dimensions. 
factor loading above or equal to the threshold for one and only one factor, and each factor should include at least three items. This way, I eliminated nine items (having desires, understanding how others are feeling, having intentions, holding beliefs, experiencing pride, perceiving depth, having free will, remembering things, communicating with others) that have factor loadings below 0.4 or have eligible factor loadings for multiple factors.

All the items in the first dimension would seem to be negative feelings, experiences, or physiological states such as feeling tired, feeling depressed, experiencing pain, and getting hungry. ${ }^{73}$ Hence, I labelled this dimension as "Negative State". The second dimension is the opposite and includes only positive states such .as feeling happy and experiencing joy. ${ }^{74} \mathrm{I}$ labelled this dimension as "Positive State". The third dimension, where I labelled "Perception and Computation", includes all the perceptual states (seeing things, detecting sounds, detecting odours and sensing temperature), except perceiving depth whose factor loading (0.38) is very close to the threshold, as well as doing computations and recognizing someone. The fourth and final dimension, which I labelled "Agency and Consciousness", includes the mental capabilities related to agency, consciousness and reasoning such as telling right from wrong, having a personality and being self-aware. ${ }^{75}$ Table 1 shows the factor loadings for each item and the dimension under which they fall. ${ }^{76}$ The factor loadings greater than the threshold were indicated in bold. The color of the

73 This dimension includes feeling tired, feeling depressed, feeling nauseated, feeling embarrassed, feeling disrespected, experiencing pain, experiencing fear, experiencing guilt, getting angry and getting hungry.

74 This dimension includes feeling happy, feeling calm, feeling safe, feeling love, experiencing joy, and experiencing pleasure.

75 This dimension includes exercising self-restraint, reasoning about things, telling right from wrong, making choices, working toward a goal, having thoughts, being conscious, having a personality and being self-aware.

${ }^{76}$ I also conducted a Hierarchical Cluster Analysis using the Ward method and euclidean distance measure to see if it would produce the similar clusters. The cluster analysis suggested the same dimensions as the exploratory factor analysis. Since I used the same questions in Weisman et al. (2017), although the presented study did not aim to determine the dimensions of lay perception of mind per se, it is still worth noting the similarities and differences between these results and the results of Weisman et al. (2017). Like the Mind dimension in Weisman et al. (2017), the perception dimension includes all the perceptual states except "perceiving depth". However, making choices, working toward a goal, reasoning about things are subsumed under Agency and Consciousness dimension in this study. Like the Heart dimension in Weisman et al. (2017), having thoughts, having a personality, telling right from wrong and exercising self-restraint fall under the same dimension (Agency and Consciousness) in the current study. However, 
dimensions and the items they subsume match. The colorless items are the ones falling under no dimension according to the above-mentioned item-selection criteria.

\begin{tabular}{|c|c|c|c|c|}
\hline & $\begin{array}{l}\text { Negative } \\
\text { State }\end{array}$ & $\begin{array}{l}\text { Positive } \\
\text { State }\end{array}$ & $\begin{array}{l}\text { Perception and } \\
\text { Computation }\end{array}$ & $\begin{array}{c}\text { Consciousness and } \\
\text { Agency }\end{array}$ \\
\hline feeling depressed & 0.79 & -0.23 & -0.09 & 0.10 \\
\hline feeling embarrassed & 0.77 & 0.06 & 0.01 & -0.07 \\
\hline experiencing fear & 0.74 & 0.12 & 0.09 & -0.08 \\
\hline experiencing guilt & 0.69 & 0.06 & 0.09 & 0.02 \\
\hline feeling tired & 0.69 & 0.02 & -0.1 & 0.17 \\
\hline getting angry & 0.66 & -0.01 & 0.07 & -0.01 \\
\hline feeling disrespected & 0.65 & -0.03 & 0.09 & -0.01 \\
\hline experiencing pain & 0.64 & 0.17 & 0.09 & 0.00 \\
\hline feeling nauseated & 0.61 & 0.02 & 0.11 & 0.00 \\
\hline getting hungry & 0.51 & 0.26 & -0.04 & 0.06 \\
\hline experiencing pleasure & 0.22 & 0.67 & 0.02 & -0.01 \\
\hline feeling love & 0.13 & 0.68 & 0.01 & 0.00 \\
\hline feeling happy & -0.02 & 0.91 & 0.01 & -0.02 \\
\hline feeling calm & -0.17 & 0.61 & 0.04 & 0.18 \\
\hline experiencing joy & 0.08 & 0.76 & 0.00 & 0.04 \\
\hline feeling safe & -0.17 & 0.51 & 0.08 & 0.26 \\
\hline detecting odours & 0.10 & 0.08 & 0.56 & 0.05 \\
\hline doing computations & -0.09 & -0.01 & 0.47 & 0.06 \\
\hline sensing temperature & 0.07 & -0.03 & 0.74 & -0.06 \\
\hline detecting sounds & 0.04 & 0.04 & 0.61 & -0.04 \\
\hline seeing things & 0.03 & 0.09 & 0.41 & 0.10 \\
\hline recognizing someone & 0.03 & -0.08 & 0.61 & 0.10 \\
\hline exercising self-restraint & 0.08 & -0.03 & 0.13 & 0.55 \\
\hline reasoning about things & 0.04 & -0.09 & 0.27 & 0.57 \\
\hline telling right from wrong & 0.02 & 0.10 & 0.16 & 0.43 \\
\hline making choices & -0.20 & 0.25 & 0.18 & 0.43 \\
\hline working toward a goal & -0.27 & 0.14 & 0.07 & 0.56 \\
\hline having thoughts & 0.39 & 0.11 & 0.00 & 0.42 \\
\hline
\end{tabular}

the other items in the Heart dimension such as experiencing pride, feeling embarrassed and feeling love and feeling disrespected fall under either Positive State or Negative State dimensions depending on whether the item is positive or negative. Similarly, Positive State and Negative State dimensions include positive and negative items that fall under the Body dimension in Weisman et al. (2017). 


$\begin{array}{lcccc}\text { being conscious } & 0.27 & 0.06 & 0.10 & \mathbf{0 . 5 2} \\ \text { having a personality } & 0.09 & 0.31 & -0.04 & \mathbf{0 . 4 5} \\ \text { being self-aware } & 0.09 & 0.13 & -0.02 & \mathbf{0 . 5 5} \\ \text { having desires } & 0.39 & 0.34 & 0.29 & -0.05 \\ \text { understanding how others are feeling } & 0.31 & 0.23 & 0.28 & 0.01 \\ \text { having intentions } & 0.31 & 0.21 & 0.23 & 0.17 \\ \text { holding beliefs } & 0.16 & 0.21 & 0.36 & 0.01 \\ \text { experiencing pride } & 0.14 & 0.30 & 0.34 & 0.00 \\ \text { perceiving depth } & 0.14 & -0.09 & 0.38 & 0.31 \\ \text { having free will } & 0.13 & 0.32 & 0.38 & 0.00 \\ \text { remembering things } & -0.14 & -0.08 & 0.19 & 0.39 \\ \text { communicating with others } & -0.20 & 0.35 & 0.03 & 0.36\end{array}$

Table 1: Factor loadings of mental capabilities in Dimension Study 1.

Looking at the results in terms of whether laypeople have common aspect intuition, recall that if laypeople have this intuition then we would expect their judgements about so-called phenomenal states to be similar to each other and thus we would expect "phenomenal" states to fall under the same dimension. This is because the very notion of phenomenal consciousness assumes that perceptual states and bodily sensations (and more arguably feelings, emotions, moods and felt thoughts) share an important commonality: there is something that it is like to be in these states. However, the participants in the present study did not classify the items in terms of this supposed commonality. Instead, they classified mental states in terms of positive/negative valence of the states, whether they are perceptual states or not, and finally, whether they describe general or overall capabilities (making choices, reasoning, and having goals, consciousness, self-awareness and so on). The results suggest that, as predicted and as indicated by Weisman et al. (2017) and Malle (2019), SM and its follow-up studies, the participants tended to treat the perceptual states and other allegedly phenomenal states somewhat differently, with perceptual states falling under the "Perception and Computation" dimension and other so-called phenomenal states in "Positive State" or "Negative State" dimensions.

A potential worry is that if the analysis had been conducted with three factors instead of four, then phenomenal states would have fallen under the same dimension. To check this possibility, I repeated the exploratory factor analysis from above using three factors. Positive and negative states 
remained in distinct dimensions. In the three-dimensional structure, the Perception and Computation dimension stayed consistent but included new perceptual/cognitive items (perceiving depth, reasoning and remembering), while one dimension subsumed all the negative states and having thoughts and the other dimension subsumed all the positive states as well as the items previously subsumed under the Agency and Consciousness dimension. ${ }^{77}$ So, the main finding of the 4-factors analysis was repeated in the 3-factors analysis: There is a gap between perceptual and non-perceptual phenomenal states.

To summarize, as with Weisman et al. (2017) and Malle (2019), the results of the present study give us no reason to assume that there is a latent factor that can be associated with phenomenality and that influences lay judgements about various mental states or capabilities. This is because, the participants classified so-called phenomenal and non-phenomenal mental capabilities into different and consistent dimensions. That is, they did not classify the given items in terms of their phenomenality. So, again we find reason to believe that laypeople tend to treat perceptual states and other so-called phenomenal states differently, which in turn suggests that laypeople do not share common aspect intuitions. As such, the results of the present study again support Premise 21.

However, one might object to this interpretation of the results of the studies discussed in this chapter along lines similar to that seen above with the Ambiguity Objection raised against SM: Unlike the cases describing certain feelings, emotions and moods, the perceptual cases in these studies (such as seeing things and perceiving things) might be argued to be such that they do not tend to trigger a phenomenal reading in people. If this is correct, then it might be further claimed that the participants did not read these cases in the phenomenal sense instead of reading them in the informational sense. So, the objection goes on, it is no wonder that the perceptual cases and emotions, feelings and moods were classified differently in these studies. So, it is not warranted to

\footnotetext{
${ }^{77}$ Cluster analysis with 3 clusters indicated almost the same dimensions with the exception that "having thoughts" and "perceiving depth" fell under the same dimension that include only positive states.
} 
think that the participants classified perceptual phenomenal states and non-perceptual phenomenal states differently based on these results.

There are two main reasons to resist this objection. First, in Chapters 4 and 5, we have already seen many reasons to doubt the Ambiguity Objection. So, I will not rehash all those reasons here. Second, remember that, in Malle's studies, feeling temperature and feeling touch, which are arguably more likely to trigger phenomenal reading than the other perceptual items because of their reference to feeling, fell under the dimension of Reality Interaction while other allegedly phenomenal states were subsumed under the Affect dimension. So, we have little reason to suspect that the results would be significantly different if more clearly "phenomenal" items (such as "experiencing colors") were used in these studies. Nonetheless, to assess this objection more directly, I conducted a second study on the dimensions of mind perception.

\subsection{Dimension Study 2}

Dimension Study 2 used the same vignette, recruitment method and restrictions as in the previous study. However, this time participants were asked about a different set of mental states and capacities. To test the above-mentioned objection to my interpretation of the results of Weisman et al. (2017), Malle (2019) and the Dimension Study 1, the study involved three states that can be hardly seen as insufficient to trigger a phenomenal reading if laypeople possess such a concept.

In the study, each participant was asked to rate their agreement with 25 statements about the robot running a simulation of the brain of the respondent. The vignette and the questions were as follows:

Imagine that in the future scientists are able to exactly scan a person's brain at the molecular level. Using the information from the brain scan they can create a perfect computer simulation of the working brain. They can then embed that computer in a robot body to create a robot version of the person.

Imagine that scientists scan your brain and use that information to create a robot version of you. What, if anything, do you think this robot would be capable of? 
On a scale of $1-7$, one being totally disagree and seven being totally agree, how much do you agree with each of the following claims?

1. The robot would feel pain when [he/she/it] is injured.

2. The robot would see colors.

3. The robot would experience sights and sounds.

4. The robot would make choices.

5. The robot would understand English.

6. The robot would give meaningful replies to questions in English.

7. The robot would display creativity.

8. The robot would think.

9. The robot would solve problems.

10. The robot would be capable of morality.

11. The robot would have dreams when [he/she/it] sleeps.

12. The robot would have free will.

13. The robot would have emotions.

14. The robot would have moods.

15. The robot would have self-consciousness.

16. The robot would have a personality. 
17. The robot would be intelligent.

18. The robot would be alive.

19. The robot would be conscious.

20. The robot would be aware of [him/herself].

21. The robot would be aware of things around [him/her].

22. The robot would be responsible for [his/her] actions.

23. The robot would deserve human rights.

24. The robot would have goals and ambitions.

25. The robot would have memories of past events.

The questions were presented in random order. Following these questions, the participants were asked the same attention question used in the Ambiguity Studies. As with other studies, the participants who failed the attention check were eliminated.

Note that the first three questions as well as the 13th and 14th questions involve references to the mental states (feeling pain, seeing colors, experiencing sights and sounds, emotions, and moods, in that order) that can be thought of as phenomenal states. Note also that seeing colors and experiencing sights and sounds can be expected to trigger phenomenal reading assuming that laypeople have a conception of phenomenality. So, if the objection given above to the interpretation of the previous results is accurate, we should expect no gap between perceptual states (seeing colors and experiencing sights and sound) and other so-called phenomenal states (feeling pain, emotions, and moods). 
134 responses were included in the analysis. ${ }^{78} 25$ one-sample t-tests were conducted using the Holm-Bonferroni correction to compare the mean response for each item to the neutral point. The participants tended to endorse all the statements except feeling pain, having dreams, having emotions, being alive and deserving human rights. ${ }^{79}$ Focusing on so-called phenomenal items, in

78193 eligible participants failed or did not answer the attention check. $45.5 \%$ of participants (included in the analysis) were native English-speakers, $71.6 \%$ women, aged from 16 to 60 (average 23.1 years). ${ }^{78}$

${ }^{79}$ Pain: $M=3.79, N=134, S D=2.35, t(133)=-1.03, p=1 ; 46.3 \%(N=62)$ answering 1,2, or $3, \chi^{2}=0.75, d f=1$, $p=0.39$.

Colors: $M=6.07, N=134, S D=1.48, t(133)=16.17, p<.001 ; 88.1 \%(N=118)$ answering 5,6, or $7, \chi^{2}=77.64, d f$ $=1, p<.001$.

Sights \& Sounds: $M=5.75, N=134, S D=1.65, t(133)=12.26, p<.001 ; 80.6 \%(N=108)$ answering 5,6 , or $7, \chi^{2}$ $=50.18, d f=1, p<.001$.

Choices: $M=5.34, N=134, S D=1.73, t(133)=8.99, p<.001 ; 74.6 \%(N=100)$ answering 5,6, or $7, \chi^{2}=32.51, d f$ $=1, p<.001$.

Understands English: $M=6.35, N=134, S D=1.23, t(133)=22.05, p<.001 ; 90.3 \%(N=121)$ answering 5,6 , or 7 , $\chi^{2}=87.04, d f=1, p<.001$.

Reply in English: $M=5.77, N=134, S D=1.47, t(133)=13.92, p<.001 ; 81.3 \%(N=109)$ answering 5,6 , or $7, \chi^{2}$ $=52.66, d f=1, p<.001$.

Creativity: $M=5.1, N=134, S D=1.66, t(133)=7.7, p<.001 ; 69.4 \%(N=93)$ answering 5,6, or $7, \chi^{2}=20.18, d f$ $=1, p<.001$.

Think: $M=5.26, N=134, S D=1.87, t(133)=7.8, p<.001 ; 70.9 \%(N=95)$ answering 5,6, or $7, \chi^{2}=23.4, d f=1$, $p<.001$.

Solve Problems: $M=5.63, N=134, S D=1.35, t(133)=13.92, p<.001 ; 83.6 \%(N=112)$ answering 5,6 , or $7, \chi^{2}=$ $60.45, d f=1, p<.001$.

Morality: $M=4.76, N=134, S D=1.98, t(133)=4.45, p<.001 ; 59 \%(N=79)$ answering 5,6, or $7, \chi^{2}=4.3, d f=1$, $p=0.038$.

Dreams: $M=3.95, N=134, S D=2.27, t(133)=-0.27, p=1 ; 41.8 \%(N=56)$ answering 1,2, or $3, \chi^{2}=3.61, d f=1$, $p=0.057$.

Free will: $M=4.55, N=134, S D=2.09, t(133)=3.06, p=0.016 ; 56.7 \%(N=76)$ answering 5,6, or $7, \chi^{2}=2.42, d f$ $=1, p=0.12$.

Emotions: $M=4.46, N=134, S D=2.26, t(133)=2.37, p=0.096 ; 56.7 \%(N=76)$ answering 5,6, or $7, \chi^{2}=2.42, d f$ $=1, p=0.12$.

Moods: $M=4.66, N=134, S D=2.19, t(133)=3.46, p=0.0050 ; 55.2 \%(N=74)$ answering 5,6, or $7, \chi^{2}=1.46, d f$ $=1, p=0.23$.

Self-consciousness: $M=4.75, N=134, S D=2.03, t(133)=4.29, p<.001 ; 55.2 \%(N=74)$ answering 5,6 , or $7, \chi^{2}=$ $1.46, d f=1, p=0.23$.

Personality: $M=5.09, N=134, S D=1.95, t(133)=6.47, p<.001 ; 68.7 \%(N=92)$ answering 5,6, or $7, \chi^{2}=18.66$, $d f=1, p<.001$.

Intelligent: $M=5.91, N=134, S D=1.16, t(133)=19.07, p<.001 ; 88.1 \%(N=118)$ answering 5,6, or $7, \chi^{2}=77.64$, $d f=1, p<.001$.

Alive: $M=4.17, N=134, S D=2.29, t(133)=0.87, p=1 ; 45.5 \%(N=61)$ answering 5,6, or $7, \chi^{2}=1.07, d f=1, p$ $=0.3$.

Conscious: $M=4.84, N=134, S D=2.05, t(133)=4.73, p<.001 ; 59.7 \%(N=80)$ answering 5,6, or $7, \chi^{2}=5.04, d f$ $=1, p=0.025$. 
line with SM and its follow-up studies, pain received the lowest average rating $(M=3.79)$ while seeing colors received the second-highest average rating $(M=6.07)$. The mean response for the other perceptual case, namely experiencing sights and sound, was also one of the statements that received a high average rating $(M=5.75)$. Unlike feeling pain, the mean responses for emotions

Self-awareness: $M=4.98, N=134, S D=1.86, t(133)=6.08, p<.001 ; 64.9 \%(N=87)$ answering 5,6, or $7, \chi^{2}=$ $11.94, d f=1, p<.001$.

Awareness of environment: $M=5.68, N=134, S D=1.59, t(133)=12.24, p<.001 ; 78.4 \%(N=105)$ answering 5 , 6 , or $7, \chi^{2}=43.1, d f=1, p<.001$.

Responsible for actions: $M=4.94, N=134, S D=2.01, t(133)=5.4, p<.001 ; 62.7 \%(N=84)$ answering 5,6 , or 7 , $\chi^{2}=8.63, d f=1, p=0.0033$.

Deserve human rights: $M=4.19, N=134, S D=2.36, t(133)=0.95, p=1 ; 44.8 \%(N=60)$ answering 5,6 , or $7, \chi^{2}=$ $1.46, d f=1, p=0.23$.

Ambitions: $M=4.64, N=134, S D=2.09, t(133)=3.56, p=0.0042 ; 59.7 \%(N=80)$ answering 5,6, or $7, \chi^{2}=5.04$, $d f=1, p=0.025$.

Memories: $M=5, N=134, S D=2.1, t(133)=5.51, p<.001 ; 67.9 \%(N=91)$ answering 5,6, or $7, \chi^{2}=17.19, d f=$ $1, p<.001$.

We found that there was a significant, positive, but negligible correlation between "choices" and CRT 2 scores (but not CRT), and between "think" and CRT scores (but not CRT 2). There was also a borderline significant, positive, but negligible correlation between "solve problems" and both CRT scores and CRT 2 scores. So, it is hard to say that cognitive reflections scores were a significant factor in participants' judgements about the philosophical questions:

Pain, CRT: $r=0.042, p=0.68, N=98$; CRT 2: $r=-0.14, p=0.14, N=108$.

Colors, CRT: $r=0.1, p=0.32, N=98$; CRT 2: $r=-0.03, p=0.79, N=108$.

Sights \& Sounds, CRT: $r=0.19, p=0.063, N=98$; CRT 2: $r=-0.03, p=0.79, N=108$.

Choices, CRT: $r=0.19, p=0.057, N=98$; CRT 2: $r=0.2, p=0.037, N=108$.

Understands English, CRT: $r=-0.02, p=0.87, N=98$; CRT 2: $r=0.18, p=0.055, N=108$.

Reply in English, CRT: $r=0.067, p=0.51, N=98$; CRT 2: $r=0.14, p=0.16, N=108$.

Creativity, CRT: $r=0.081, p=0.43, N=98$; CRT 2: $r=0.12, p=0.21, N=108$.

Think, CRT: $r=0.25, p=0.015, N=98$; CRT 2: $r=0.14, p=0.15, N=108$.

Solve problems, CRT: $r=0.3, p=0.0023, N=98$; CRT 2: $r=0.29, p=0.0026, N=108$.

Morality, CRT: $r=0.067, p=0.52, N=98$; CRT 2: $r=0.07, p=0.47, N=108$.

Dreams, CRT: $r=0.0078, p=0.94, N=98$; CRT 2: $r=-0.08, p=0.4, N=108$.

Free will, CRT: $r=0.08, p=0.43, N=98$; CRT 2: $r=-0.01, p=0.94, N=108$.

Emotions, CRT: $r=0.098, p=0.33, N=98$; CRT 2: $r=0.062, p=0.53, N=108$.

Moods, CRT: $r=0.066, p=0.52, N=98$; CRT 2: $r=0.023, p=0.81, N=108$.

Self-consciousness, CRT: $r=0.19, p=0.061, N=98$; CRT 2: $r=0.11, p=0.27, N=108$.

Personality, CRT: $r=0.034, p=0.74, N=98$; CRT 2: $r=0.17, p=0.08, N=108$.

Intelligent, CRT: $r=0.12, p=0.25, N=98$; CRT 2: $r=0.13, p=0.17, N=108$.

Alive, CRT: $r=-0.03, p=0.76, N=98$; CRT 2: $r=-0.06, p=0.56, N=108$.

Conscious, CRT: $r=0.027, p=0.79, N=98$; CRT 2: $r=0.1, p=0.29, N=108$.

Self-awareness, CRT: $r=0.068, p=0.51, N=98$; CRT 2: $r=0.18, p=0.062, N=108$.

Awareness of environment, CRT: $r=0.065, p=0.52, N=98$; CRT 2: $r=0.12, p=0.22, N=108$.

Responsible for actions, CRT: $r=0.2, p=0.053, N=98$; CRT 2: $r=0.052, p=0.59, N=108$.

Deserve human rights, CRT: $r=0.14, p=0.18, N=98$; CRT 2: $r=0.0032, p=0.97, N=108$.

Ambitions, CRT: $r=0, p=0.99, N=98$; CRT 2: $r=0.1, p=0.28, N=108$.

Memories, CRT: $r=-0.05, p=0.64, N=98$; CRT 2: $r=-0.03, p=0.77, N=108$. 
and moods were above and significantly different from the neutral point $(M=4.46$ and $M=4.66$, respectively). Figure 7 shows the mean responses of the statements that involve these allegedly phenomenal states.

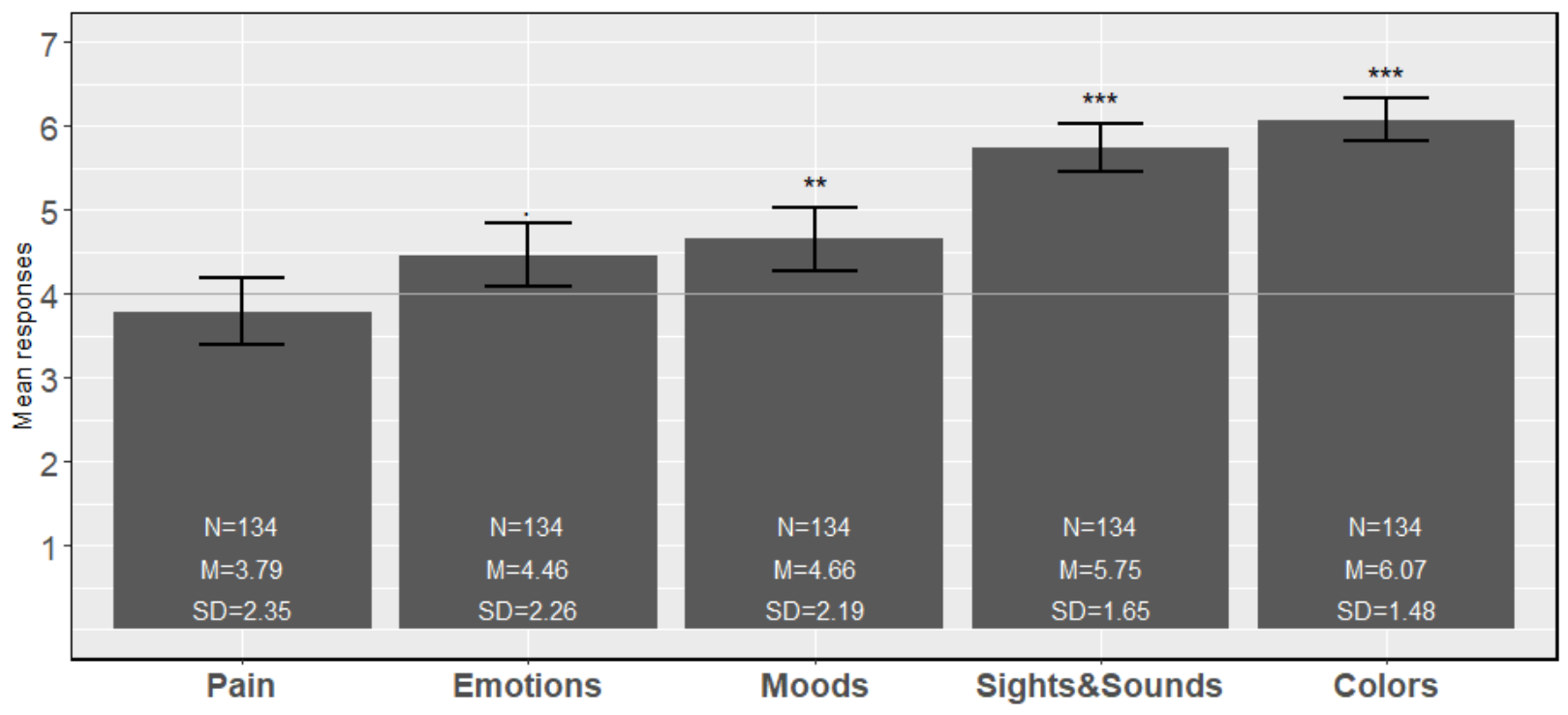

Figure 7: The mean responses for supposedly phenomenal states in Dimension Study 2.

Remember that the objection to my interpretation of the previous studies was that perceptual states are not likely to trigger phenomenal reading, and this may explain why the participants in those studies classified perceptual states and other so-called phenomenal states into different dimensions. Against this objection, in parallel to SM and its follow-up studies, the participants endorsed that the robot can see colors and experience sights and sounds, which are more likely to trigger phenomenal reading than the perceptual states involved in previous studies, but denied that it can feel pain. In keeping with this, there were significant differences (with large effect sizes) between feeling pain and both seeing colors and experiencing sights and sounds. ${ }^{80}$ As such, the study again reinforces SM's finding that laypeople treat one group of prototypical "phenomenal"

${ }^{80}$ Pain X Colors: $t(224.81)=-9.52, p<.001$, Cohen's $d=1.16$.

Pain X Sights \& Sounds: $t(238.57)=-7.89, p<.001$, Cohen's $d=0.96$. 
states differently from the other prototypical examples of so-called phenomenal states. Further, six two-sample t-tests conducted using the Holm-Bonferroni correction to compare the mean responses for non-perceptual states with the mean responses for perceptual cases suggested that the participants were significantly more likely to endorse perceptual states than non-perceptual, so-called phenomenal states. ${ }^{81}$

In supporting the above findings, the exploratory factor analysis suggested that the participants classified perceptual cases and non-perceptual "phenomenal" cases into different dimensions. Parallel analysis with minimum residuals solution suggested that 25 variables/statements can be reduced into two factors (or dimensions). ${ }^{82}$ I conducted exploratory factor analysis with two factors, minimum residual factor algorithm and oblimin rotation. ${ }^{83}$ I retained both factors as each factor accounted for at least $5.0 \%$ of variance. Applying the same threshold used in the Dimension Study $1(|0.40|)$, the analysis suggests that perceptual cases (seeing colors and experiencing sights and sounds), creativity, understand English, reply in English, making choices, solve problems, being intelligent and awareness of environment fell under the same dimension which I term "Perception and Cognition". The other dimension, which I term "Agency, Consciousness and Experience", includes non-perceptual phenomenal states (feeling pain, emotions and moods) together with other capabilities related to consciousness and agency such as conscious, selfconsciousness, self-awareness, free will, personality and responsibility for actions. ${ }^{84}$ Memories

\footnotetext{
${ }^{81}$ Colors X Pain: $t(224.81)=-9.52, p<0.001$, Cohen's $d=1.16$. Colors X Emotions: $t(229.79)=6.90, p<.001$, Cohen's $d=0.84$. also suggested the same factor count. The scree plot of principle component analysis also suggested 2 factors.

${ }^{83}$ Factor analysis with minimum residual solution and different rotation methods such as varimax and promax resulted in similar dimensions. The analysis with maximum likelihood solution and oblimin rotation also indicated the similar dimensions.

${ }^{84}$ Hierarchical Cluster Analysis using the Ward method with a Euclidean distance measure classified 24 items into the same dimensions as in the factor analysis.
} 
was not classified in any dimension as both factor loadings for this item was under the threshold. Table 2 lists the factor loadings of each statement for both dimensions.

\begin{tabular}{lcc} 
& Consciousness and Agency & Perception and Cognition \\
\hline Pain & $\mathbf{0 . 8 9}$ & -0.14 \\
Think & $\mathbf{0 . 5 5}$ & 0.21 \\
Morality & $\mathbf{0 . 6 3}$ & 0.22 \\
Dreams & $\mathbf{0 . 7 5}$ & -0.07 \\
Free will & $\mathbf{0 . 6 5}$ & 0.16 \\
Emotions & $\mathbf{0 . 8 8}$ & -0.02 \\
Moods & $\mathbf{0 . 7 4}$ & -0.02 \\
Self-consciousness & $\mathbf{0 . 8 3}$ & 0.02 \\
Personality & $\mathbf{0 . 5 2}$ & 0.30 \\
Alive & $\mathbf{0 . 8 3}$ & -0.08 \\
Conscious & $\mathbf{0 . 7 6}$ & 0.12 \\
Self-awareness & $\mathbf{0 . 6 7}$ & 0.12 \\
Responsible for actions & $\mathbf{0 . 6 0}$ & 0.10 \\
Deserve human rights & $\mathbf{0 . 8 7}$ & -0.16 \\
Ambitions & $\mathbf{0 . 6 2}$ & 0.27 \\
Colors & 0.28 & $\mathbf{0 . 4 8}$ \\
Sights \& Sounds & 0.37 & $\mathbf{0 . 4 0}$ \\
Choices & 0.32 & $\mathbf{0 . 5 0}$ \\
Understand English & 0.00 & $\mathbf{0 . 6 7}$ \\
Reply in English & 0.02 & $\mathbf{0 . 6 5}$ \\
Creativity & 0.14 & $\mathbf{0 . 4 8}$ \\
Solve problems & -0.09 & $\mathbf{0 . 7 9}$ \\
Intelligent & -0.15 & $\mathbf{0 . 5 8}$ \\
Awareness of environment & 0.14 & $\mathbf{0 . 6 6}$ \\
Memories & 0.38 & 0.26 \\
& &
\end{tabular}

Table 2: Factor loadings of 25 statements in Dimension Study 2.

Comparing the dimensions this study suggests with the dimensions suggested by the previous study, we see a simpler picture here in which the states were distinguished from each other in terms of whether the states are perceptual/cognitive states or not. This is probably because of the smaller range of the items. For the current purpose of the chapter, what is more important is that the key finding in the previous study is confirmed by the dimensions the current study suggests: perceptual and non-perceptual phenomenal states fall under different, consistent, and meaningful dimensions. 
Although the factor loading of seeing colors (0.48) is close to the threshold and the factor loading of sights \& sounds (0.40) is on the borderline, the differences between the factor loadings of these three states for the dimension of Agency, Consciousness and Experience and Perception and Cognitions are notable. As can be seen from Table 2, pain, having emotions and having moods stand in negative correlation with the Perception and Cognition dimension. The magnitude of this separation of non-perceptual "phenomenal" items from the dimension of Perception and Cognition can be seen more easily from Figure 8 . The figure describes a two-dimensional coordinate system in which the $\mathrm{X}$ and $\mathrm{Y}$-axis represent the Perception and Cognition and Agency, Consciousness and Experience dimensions. Each item is located according to its factor-loadings for both dimensions. As such, the top-left area includes the items of Perception and Cognition dimension while the bottom-right area includes the items of Agency, Consciousness and Experience dimension. Note that pain and emotions are the rightmost items (i.e. the items with the lowest factor loadings for Perception and Cognition), and having moods is relatively further to the Perception and Cognition dimension compared to for example personality, ambitions, thinking, and morality.

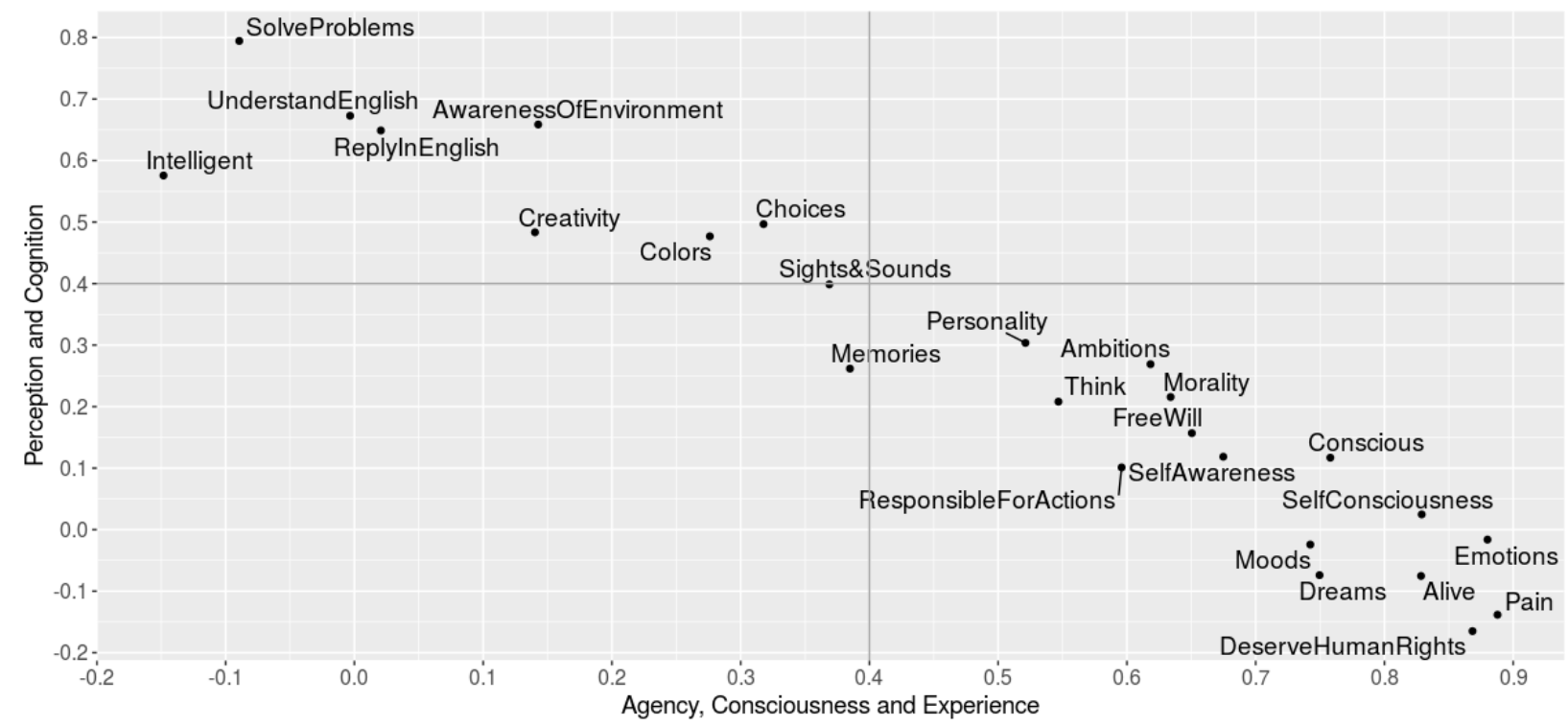

Figure 8: The coordinates of mental capabilities in two dimensions indicated in Dimension Study 2. 
One can also argue, again along the lines of the Ambiguity Objection, that "seeing colors" might be ambiguous between phenomenal and informational reading. However, it should be noted that even if "seeing colors" would not be sufficient to trigger a phenomenal reading if people have such a concept, it seems far-fetched to claim the same for experiencing sights and sounds. After all, "experience" is a term that philosophers typically take to be synonymous with phenomenally conscious states. So, if laypeople had a conception of phenomenality, we would expect them to treat experiencing sights and sounds and other supposed phenomenal states similarly. However, they did not! As noted, experiencing sights and sounds was given significantly higher ratings than non-perceptual phenomenal states.

In brief, the results of the study do not bear out the prediction of the objector because seeing colors and experiencing sights and sounds were still treated significantly differently from pain, emotions and moods, and were classified into a different dimension from the dimension of emotions, moods, and pain. This study, as with Weisman et al. (2017), Malle (2019) and Dimension Study 1, suggests that laypeople do not share common aspect intuitions by which they treat phenomenal states similarly.

\subsection{A General Objection}

In this section, I consider a general objection to the interpretation I've offered of the results of Weisman et al. (2017), Malle (2019) and Dimension Studies 1-2: One could have a concept but depending on the question, her judgements may or may not produce dimensions that divide along the lines of that concept. For example, if one is asked which shapes are more common in the world, one's responses may form two dimensions. One dimension may include circles, squares, and triangles, while the other may include ellipses and trapezoids considering that circles, squares, and triangles are more common than the others. However, the objection goes on, this does not mean that one has no concept of ROUNDNESS just because circles and ellipses fall under into different dimensions.

It is obviously true that the dimensions are formed depending on the questions. If the questions do not concern the distinctive features of a concept, we should not expect that a dimension 
consistently includes the items with those features. In the example above, note that "being more common in the world" is not a distinctive feature of shapes. If the question on the other hand refers to the distinctive feature of shapes such as their appearances (i.e. "which shapes seem similar to each other?"), we should expect people to classify circles and ellipses in one dimension.

When we look at the questions asked in the studies we discussed in this chapter, however, we see that the questions discussed refer to a distinctive feature of phenomenality. For example, the question of whether an agent can see colors or feel pain concerns colors and pains which most philosophers consider as distinctive features of two paradigmatic phenomenal states. So, if the participants shared common aspect intuitions, they would tend to classify seeing colors and feeling pain but not non-phenomenal states into the same dimension (just as they would tend to classify circles and ellipses but not squares and triangles into the same dimension when they are asked about how these shapes appear). In the same vein, they would do the same for other so-called phenomenal experiences. So, the dimensions resulted from the responses to the questions discussed in this chapter would be expected to elicit a distinction between phenomenal states and non-phenomenal states if people draw such a distinction.

To put the same point another way, consider the following analogy. We can imagine a study investigating the question of whether 5-year-old children have tacit or explicit concepts of ANIMAL and PLANT in which the children are asked to rate their agreement with the following statements: "a bird can fly", "a flower can move from one place to another", "a crocodile has leaves", "a fish feeds on sunlight", "an oak tree has roots", "a human is rooted in place", "flowers can escape from harm", "a flower can fly", "a living-thing in your living room rooted in the soil can produce eggs", "the things that can fly can produce eggs" and so on. Since the questions concern the distinctive features of animals and plants (fly/move/escape from harm and produce eggs for animals, and have roots/leaves and feeding-on-sunlight for plants), it is safe to assume that if a child has a conception of ANIMAL or PLANT, we would expect her to ascribe flying, moving, escaping from harm and other characteristic features of animals to the animals while denying ascribing having leaves, having roots, being rooted in soil to them. We would also expect her to do the opposite for plants. As such, exploratory factor analysis and cluster analysis would 
be expected to indicate two dimensions or clusters. What this analogous example helps illustrate is that if the questions concern the distinctive features related to a concept, we would expect the resultant dimensions to reflect the distinction between the features that can be applied to a concept and the features that cannot.

So, we have a good reason to think that if laypeople had a conception of phenomenality, phenomenal states, at least the prototypical ones, would be in the same dimension in the discussed studies. Since so-called phenomenal states fall under different dimensions in these studies, it is safe to assume that laypeople treated these states notably differently, which would cast considerable doubt on the common aspect claim - that laypeople recognize the common aspect (qualitativeness/what-it-is-likeness) in different phenomenal experiences (i.e. they have common aspect intuitions).

\subsection{Concluding Remarks}

Studies on the dimensions of the lay conception of mind provide insights into the question of whether laypeople treat prototypical examples of phenomenal cases similarly or not. We have seen that laypeople classify perceptual cases differently from other non-perceptual, so-called phenomenal cases based on the results of Weisman et al. (2017), Malle (2019) and in Dimension Studies 1-2, which cast further doubt on the common aspect claim. This is because, if people had this intuition, they would tend to classify perceptual "phenomenal" cases (seeing, hearing, and so on) and other phenomenal cases (feeling pain, emotions, moods and so on) into the same dimension. However, the studies discussed provided us with a clear picture in which perceptual cases and other so-called phenomenal states fall under different, consistent, and meaningful dimensions in the lay conceptualization of mind. Further, even if we ignore the dimensions these studies suggest, some studies discussed in the chapter still give us further reason to doubt the common aspect claim. Remember the participants in Weisman et al. (2017) endorsed that a robot has perceptual capabilities such as sensing temperatures, seeing things and perceiving depth while

they denied ascribing other "phenomenal" items to it, which clearly accords with SM's key finding. Similarly, in Dimension Study 2, the participants by and large were happy with ascribing 
perceptual cases to a robot while being reluctant to attribute feeling pain to it, just as in SM and its follow-up studies. So, overall, the findings discussed in this chapter suggests against the common aspect claim, and thus support Premise 2-1, by indicating that laypeople do not seem to recognize any "phenomenal" commonality between perceptual states and bodily sensations, emotions, and moods.

In Chapters 4 and 5, I emphasized the fact that laypeople in various studies gave positive and negative answers, respectively, to the questions of whether a simple robot can see colors and feel pain. In line with this, both the existing and new studies discussed in this chapter suggest that laypeople treat perceptual cases and other so-called phenomenal states differently. Taken these three chapters together, there seems no reason for us to believe the common aspect claim, and in fact provide good reason to deny it: by and large laypeople do not seem to have common aspect intuitions reflecting that perceptual states and bodily sensations (and more controversially emotions, moods, felt thoughts) share a common, what-it-is-like aspect. That is, we have good reason to think that laypeople by and large do not share common aspect intuitions. Remember also that the studies discussed in $\$ 4.1$ suggest that laypeople do not share most philosophers’ experiential property intuitions that reflect that the properties we are acquainted with in perceptual states and bodily sensations are the properties of our experiences, either. So, we can safely conclude from the empirical evidence discussed in these chapters that the current evidence provides strong support for Premise 2-1. To fully establish Premise 2, though, we also need to show that the evidence also supports Premise 2-2. That is, we need evidence indicating that epistemic gap intuitions are not prevalent either, which the next two chapters provide. 


\section{PREVALENCE OF EPISTEMIC GAP INTUITIONS IN THE EXISTING LITERATURE}

Premise 2 of the General Argument holds that the intuitions used to defend phenomenal realism and the epistemic gap thesis are not prevalent. This can be broken down into the following two sub-premises:

Premise 2-1: Existence intuitions (the intuitions that individually or collectively suggest that phenomenal consciousness exists, i.e. the intuitions that are suggestive of phenomenal realism) are not prevalent.

Premise 2-2: Epistemic gap intuitions (the intuitions that are suggestive of the epistemic gap between the phenomenal and the physical) are not prevalent.

Chapters 4-6 made the case that Premise 2-1 is true. Chapters 7-8 aim to do the same for Premise 2-2 by showing in this chapter that the existing evidence suggests that laypeople do not share epistemic gap intuitions and then introducing new experimental studies in the next chapter that cast further doubts on epistemic gap intuitions being widespread.

As noted in Chapter 3, there are three major kinds of epistemic gap intuitions: conceivability intuitions reflecting that it is conceivable that the phenomenal and the physical come apart, the knowledge intuition reflecting that knowing every physical fact would not be enough to know all the phenomenal facts, and the explanatory gap intuition reflecting that no amount of physical knowledge would suffice to explain phenomenal consciousness. In this chapter, I will examine the prevalence of conceivability and explanatory gap intuitions by discussing existing studies.

\$7.1 discusses the prevalence of conceivability intuitions in light of the first study in Sytsma (2010), the second study in Peressini (2014) and Study 4 in Fischer and Sytsma (2021). \$7.2 discusses the results of Gottlieb and Lombrozo (2018) and Díaz (2021) to evaluate the prevalence of explanatory gap intuitions. The chapter concludes that the existing studies about the conceivability and explanatory gap intuitions suggest that these intuitions are far from being widespread. 


\subsection{Prevalence of Conceivability Intuitions}

As noted, conceivability intuitions, which Chalmers (2018) calls "modal intuitions" (p. 12), reflect that it is conceivable that the physical and the phenomenal split from each other. And as stated in $\S 3.4$, conceivability intuitions include philosophical zombie intuitions and what Chalmers (2018, p. 12) calls "inversion intuitions" suggesting for the conceivability of inverted spectrum cases. This section will first discuss the prevalence of inversion intuitions in light of the first study in Sytsma (2010), and then the prevalence of philosophical zombie intuitions by looking at the results of the second study in Peressini (2014) as well as Study 4 of Fischer and Sytsma (2021).

\subsubsection{Inverted Spectrum Case}

As we have seen in $\$ 4.1$, the first study in Sytsma (2010) includes a question that describes an inverted spectrum case. The question read as follows:

Do you think it is possible that somebody else might actually see the color that you call 'blue' when they look at an ordinary ripe tomato, despite having normal visual acuity (i.e., without being color-blind)? (p. 120).

Remember that the mean response to this question was significantly below the neutral point. Remember also that since the inverted spectrum case defined above is possible only if colors are properties of experiences rather than properties of the perceived object or light (given the same capabilities of sight and the same optic conditions), the mean response suggests that laypeople do not share experiential property intuitions. Apart from that, this evidence has a more direct implication for the prevalence of the inversion intuitions because it straightforwardly suggests that laypeople do not tend to find inverted spectrum cases possible. 
However, it is not clear that which sense of possibility (logical or metaphysical possibility) the participants had in their minds while answering the question. ${ }^{85}$ If they took the term "possible" in the sense of logical possibility, this would suggest that they found the inverted spectrum cases inconceivable. But if they distinguish the logical possibility from the metaphysical possibility and if they had metaphysical possibility in their minds while answering the questions, then it would not be clear that they find inverted spectrum cases inconceivable. Considering this, can we say that the participants generally found the described inverted spectrum case inconceivable and thus that laypeople by and large lack the inversion intuitions just because the mean response to the question in this study was significantly below the neutral point? As will be seen in the next two sub-sections, a similar question can be asked for philosophical zombie intuitions. I will answer this question concerning both kinds of intuitions in $\$ 7.1 .4$ after discussing the evidence regarding the prevalence of philosophical zombie intuitions.

\subsubsection{Zombie Questions in Peressini's Study}

As noted before, in his second study, Peressini (2014) asked ten questions to investigate the characteristics of the lay conception of subjective experience. I have already discussed Peressini's two questions (the third and sixth questions) in $\$ 4.2 .3$ in terms of whether laypeople have a conception of phenomenal consciousness in the first place. Now, for the present purpose of this chapter, I will discuss the responses to Questions 1 and 8. Questions 1 and 8 were as follows:

Question 1: Imagine a medical procedure that would remove your inner experience without affecting your brain, so from the outside you would remain unchanged physically and behaviourally. Do you think such a procedure is possible? (p. 874)

\footnotetext{
${ }^{85}$ For this reason, the references to the possibility in my interpretation of this and subsequent studies that will be discussed in $\S 7.1 .2$ and $\S 7.1 .3$ will be neutral unless the exact sense of possibility is specified.
} 
Question 8: Imagine a person physically and behaviourally identical to you in all ways but who had no inner experience at all. Is such a person possible? Would such an entity be an experiencer? (p. 875)

The mean responses to these questions were 2.30 and 2.55 respectively (on a 7-point scale, anchored at 1 with "certainly not", 4 with "not sure" and 7 with "certainly so"). These figures were below and significantly different from the neutral point of 4 (p. 876).

Note that Question 8 describes the zombie scenario presented in Chalmers's philosophical zombie argument, and Question 1 is a variant of the same scenario in that it asks whether one of two identical brains can enjoy inner experiences that the other lacks. ${ }^{86} \mathrm{We}$ have seen that there are good reasons to doubt that laypeople generally have the concept of phenomenal consciousness. As such, we should not assume that they understand "inner experience" in the questions in the philosophical sense of phenomenal consciousness. But even if we set aside this worry and assume, as Peressini does, that people understand this and similar expressions as Peressini intended, the low responses to these questions clearly suggest that they find the possibility of a split between the phenomenal and the physical unrealistic. It is a different, if related, question, however, whether we can conclude from these results that laypeople think that any split between the phenomenal and the physical is inconceivable. This question will be answered in $\$ 7.1 .4$ after discussing the evidence regarding the prevalence of philosophical zombie intuitions presented in Fischer and Sytsma (2021).

\footnotetext{
86 There are two main versions of the argument presented in Chalmers (1996) and Chalmers (2009). However, the core idea remains the same and can be summed up as follows: philosophical zombies are molecule-molecule physical duplicates of humans that have functionally the same mental states and behave just like us. However, there is a big difference between us and philosophical zombies. Unlike us, they lack phenomenal experiences and thus they do not have an inner life (i.e. there is nothing it is like to be a zombie). The argument claims first that philosophical zombies are conceivable, second that if they are conceivable, then they are possible, and finally concludes that they are possible. And if they are possible, then physicalism is false because if zombies are possible then there are some facts (inexistence of phenomenal consciousness in the argument) that are not determined by the physical (i.e. the phenomenal does not supervene on the physical).
} 


\subsubsection{Fischer and Sytsma's Study}

Fischer and Sytsma (2021) hypothesise that Chalmers's philosophical zombie argument is built on "zombie intuitions"-intuitions that are killed (defeated) by contextual information but still remained cognitively alive by cognitive bias (p. 1). They then empirically test this hypothesis, presenting evidence in its favour. While the details of this hypothesis and the range of evidence brought to bear on it are interesting, for our purposes we can simply focus on their concluding experiment.

In Fischer and Sytsma's fourth study, each participant was given the following vignette, using either the word 'zombie' or 'duplicate':

Imagine that in the future scientists are able to exactly scan a person's body, including their brain, at the molecular level. Using this information, they can then create an exact physical duplicate of that person's body and brain, molecule by molecule. The resulting ['zombie'/duplicate] will have a body and brain just like the original person's. The [zombie/duplicate] will also behave just like that person. But, when it comes to the [zombie/duplicate], all is dark inside.

Imagine that scientists successfully scan and duplicate an average person in this way. What, if anything, do you think the resulting [zombie/duplicate] would be like? (p. 5)

Then, each participant was asked about three clusters of typical features that are associated with the Hollywood sense of "zombie", three clusters of atypical features of Hollywood zombies, and three items related to consciousness. ${ }^{87}$ For the purposes of this chapter, I will focus on just the consciousness-related items. These read as follows:

(A) these beings are capable of having conscious experiences

\footnotetext{
${ }^{87}$ Typical features include ("Zombies have rotting bodies and attack and eat humans", "Zombies move slowly and have lifeless faces", and "Zombies lack free will and feel no joy") while atypical features include ("Zombies feel hate and feel sad", "Zombies think and are intelligent", and "Zombies sing, smell flower, are happy, and feel love").
} 
(B) these beings have an inner mental life, including feelings and emotions

(C) these beings are sentient and experience their surroundings and sensations.

(D) there is something it is like to be such a being.

The participants were asked to rate their agreement with these ten statements, on a 7-point scale (anchored at 1 with 'totally disagree', at 4 with 'neither agree nor disagree', and at 7 with 'totally agree'). The statements were given in random order. Each participant was given either the "duplicate" or "zombie" versions of the statements, corresponding with the version of the vignette they read. The proportion of the participants who disagreed with the statements A-D (i.e. the proportions of the participants who endorse that philosophical zombies are possible for each consciousness statement) were as follows:

\begin{tabular}{|l|l|l|l|l|}
\hline & A & B & C & D \\
\hline Zombie & $32.3 \%$ & $39.8 \%$ & $31.2 \%$ & $31.2 \%$ \\
\hline Duplicate & $20.0 \%$ & $20.0 \%$ & $15.8 \%$ & $22.1 \%$ \\
\hline
\end{tabular}

Table 3: Proportions of the participants who endorse that philosophical zombies are possible in Study 4 of Fischer and Sytsma (2021).

The authors noted that there were significant differences between the percentages of disagreement with "Zombie" and "Duplicate" statements for A-C (p. 8). They were most concerned with this difference between the conditions (using "zombie" versus "duplicate") and take this to be evidence that the use of the term "zombie" in philosophical discussions triggers a type of cognitive bias (specifically, linguistic salience bias). ${ }^{88}$ Setting this aside, however, what is most important for the present discussion is that even when using this (problematic) term, the proportion of the

${ }^{88}$ See Fischer and Engelhardt (2017) in addition to Fischer and Sytsma (2021). 
participants that we can plausibly treat as endorsing the possibility of philosophical zombies is below and significantly different from 50.0\%. And when the term "duplicate", which describes the same notion of philosophical zombie, is used in the statements, this figure is reduced still further. Even assuming there is a folk conception of phenomenal consciousness, these results clearly suggest that laypeople by and large would attribute so-called phenomenal experiences to physicobehaviourally duplicates of us. That is, they find the notion of philosophical zombie unrealistic.

\subsubsection{Do These Studies Show that Laypeople by and large Lack Conceivability Intuitions?}

We have seen that three studies discussed suggest that laypeople by and large do not find a split between the physical and the phenomenal realistic. To recapitulate, the first study in Sytsma (2010) suggests that laypeople tend to think that the experience of seeing colors would be the same if the relevant physical conditions were the same. That is, they find inverted spectrum cases unrealistic. And Peressini (2014) and Fischer and Sytsma (2021) suggest that laypeople find the notion of philosophical zombie unrealistic. That is, they tend to think that our physical duplicates would share our subjective experiences. This seems to suggest that laypeople do not tend to conceive a split between the "phenomenal" experiences and the physical. In other words, assuming people have a conception of phenomenality, the idea that the phenomenal and the physical might be separated is not conceivable for the majority of laypeople. And this in turn suggests that the conceivability intuition is not prevalent.

However, as noted, it is not clear which sense of possibility the participants in these studies had in their minds while answering the questions. So, perhaps the participants, by and large, found the inverted spectrum case and philosophical zombies impossible in the sense of metaphysical possibility rather than logical possibility. And it is logical possibility rather than metaphysical possibility that might suggest anything about the question of whether laypeople find a split between the phenomenal and the physical conceivable. After all, following the philosophical literature on conceivability arguments, one can claim that conceivability is one thing and the metaphysical possibility is another. Type-B physicalists, unlike Type-A materialists, for example, believe that philosophical zombies (or any split between the physical and the phenomenal in 
general) are conceivable but not metaphysically possible. ${ }^{89}$ In other words, roughly speaking, these philosophers believe that we can coherently imagine inverted spectrum cases or philosophical zombies (because these cases cannot be ruled out through a priori reasoning and thus they are logically possible) but that they are nonetheless metaphysically impossible. Perhaps, just like Type-B physicalists, the participants in these studies could coherently imagine inverted spectrum and zombie cases, but they found those cases metaphysically impossible. In this case, the results presented would be irrelevant to the prevalence of conceivability intuitions. The results at most would show that laypeople by and large are unwilling to endorse the metaphysical possibility of inverted spectrum cases and the existence of philosophical zombies. So, the objection goes, we cannot straightforwardly conclude from these results that the participants found inverted spectrum cases and zombie cases inconceivable.

However, it is crucial to see that Type-B physicalists deny the genuine, metaphysical possibility of a split between the phenomenal and the physical because of theoretical reasons. That is, on the intuitive level, these physicalist philosophers find the split between the phenomenal and the physical possible, but they explain away the possibility of such a split on the philosophical, theoretical, and argumentative level. To give but an example, Papineau, a prominent Type-B physicalist, says that "Zombies seem possible" and that "intuitively we feel that there are possible worlds-zombie worlds" but he also says that "there are a number of possible ways of explaining away the intuition of distinctness, especially for physicalists who recognize phenomenal concepts. I myself favour an explanation that hinges on the use-mention feature of phenomenal concepts, and which elsewhere I have called 'the antipathetic fallacy' ” (Papineau, 2006, p. 21).

When it comes to ordinary people, on the other hand, it would be unrealistic to expect laypeople to appeal to some theoretical reasons that explain away the apparent possibility of inverted spectrum cases or philosophical zombies. Rather, it seems more realistic to expect them to base their opinions on the prima facie possibility (or impossibility) of these cases. To put the same point differently, there seem to be two options with regard to interpreting the majority of participants

\footnotetext{
${ }^{89}$ See Chalmers (2003) for details about the main differences between Type-A and Type-B materialism.
} 
being reluctant to endorse the possibility of a split between the physical and the phenomenal: (1) it seemed to the majority of the participants that the inverted spectrum case and the zombie cases are not conceivable (not coherently imaginable), or (2) a considerable proportion of them were able to distinguish two senses of possibility; they had metaphysical possibility in their minds while answering the questions; and finally they were able to coherently conceive such scenarios but rejected that those scenarios are genuinely, metaphysically possible. Note that (2) assumes too much while (1) seems to cohere with the non-philosophical nature of folk opinions. So, we can safely assume that the participants mostly rejected the possibility of these cases because these cases did not even seem to them possible on the intuitive level, i.e. they were not even conceivable for them. In Chalmers's terms, they seem to have failed to conceive a "logically coherent world" (Chalmers, 1996, p. 47) or to "coherently imagine" (Chalmers, 2003, p. 107) a world in which the physical and the phenomenal split from each other. So, it is safe to conclude that these results suggest that laypeople by and large lack conceivability intuitions.

\subsection{Prevalence of Explanatory Gap Intuitions}

Recall that explanatory gap intuitions reflect the idea that even a complete physical explanation of the mind/brain seems to leave phenomenal consciousness unexplained. The intuition holds, for example, that even a complete explanation by natural sciences would not still be able to fully explain why we see colors in the first place and why blood seems red to us instead of blue. To investigate the prevalence of explanatory gap intuitions, I will first look at two comprehensive works in experimental philosophy: Gottlieb and Lombrozo (2018) and Díaz (2021).

\subsubsection{Gottlieb and Lombrozo's Study}

A straightforward way to investigate the prevalence of explanatory gap intuitions is to ask ordinary people to rate the degree to which they think phenomenal experiences can be fully explained one day by science. Gottlieb and Lombrozo (2018) conducted two experiments along these lines. In their first study, they asked each participant first to rate their agreement with a statement that associates each of 46 psychological phenomena with one of six dimensions (introspectionphenomenology, human uniqueness, abnormal functioning, conscious will, the importance for 
identity and complexity). Some participants, for example, rated their agreement with the (human uniqueness) statement "__ is unique to humans" for each of the 46 phenomena. ${ }^{90}$ Then each participant rated their agreement with the scientific possibility statement ("Science could one day fully explain ___") for each phenomenon. They also rated the degree of their discomfort with a possible scientific explanation of each item.

Of the six above-mentioned dimensions, the relevant one for the purpose of investigating the prevalence of explanatory gap intuitions is the introspection-phenomenology dimension. The statement for this dimension was "__ involves a subjective experience (a feeling of what it is like) that only the individual experiencing it can know". The authors found an inverse correlation between the means of the ratings given for the possibility of scientific explanation and the means of the ratings given for introspective-phenomenology. Chalmers interprets this as suggesting that "people judge that phenomena tied to subjective experience and to privileged access are relatively hard to explain" (Chalmers, 2018, p. 14). Since he sees the work of Gottlieb and Lombrozo as a relevant work on explanatory gap intuitions (Chalmers, 2018, p. 14), it is clear that he thinks that, for laypeople, "phenomena tied to subjective experience and to privileged access" refer roughly to phenomenal experiences.

Again, the previous studies cast considerable doubt on the existence of a folk conception of phenomenality. Setting that aside for the sake of argument, there is still the question of whether we should expect the introspective-phenomenology dimension to be interpreted in the way (assuming that people do have a concept of phenomenal consciousness) Chalmers assumes by the participants. The assumption, in other words, is that the ratings given for each phenomenon in the introspective-phenomenology dimension at least roughly accord with its being a phenomenal

\footnotetext{
${ }^{90}$ These phenomena included perceptual states (such as perceiving depth, sense of touch, vision and hearing), feelings (feelings of jealousy/pride/guilty, pleasure from food, and so on), cognitive abilities (such as recognizing faces, using language to communicate, logical reasoning, creativity, ability to read a map), non-veridical mental states (hallucinations, dreaming, day dreaming), psychological disturbances (schizophrenia, anxiety disorders, depression), morality (making moral judgments, choosing to give to charity, acting morally, one's making own choices, altruistic acts, and so on) and many other phenomena such as believing in god, falling in love, and having an addiction.
} 
experience. So, let's first look at which phenomena got high scores for the introspectionphenomenology dimension to assess this assumption.

32 phenomena had a mean that is above the neutral point of 4. Among them, there were several items (such as feelings, depression, and pleasure from food) that can be considered as phenomenal experiences. However, there were also many items that are not typically considered as phenomenal experiences (or subjective experience in general) such as making moral judgements, and logical reasoning. As such, there is a non-negligible disaccord between the phenomena the participants considered as falling under the introspection-phenomenology dimension and "the phenomena tied to subjective experience and to privileged access". So, pace Chalmers, the inverse correlation the authors found cannot be interpreted in terms of phenomenal consciousness and thus explanatory gap.

Further, if the participants tended to read the statement for introspective-phenomenology as indicating phenomenal consciousness and if they had explanatory gap intuitions, the results would show that they by and large think that the overwhelming majority of the cases in this dimension cannot be explained by science. Among the 32 items, which had higher means than the neutral point, 30 items had a mean above the neutral point for scientific explanation. The remaining two items were believing in God and being able to feel and identify different types of textures with the sense of touch. (The means for these items were 3.67 and 3.88, respectively). Note that believing is not typically considered as a phenomenal state and thus the low mean rating for this item can be explained by reasons that have nothing to do with phenomenal consciousness. The other item describes an ability related to a "phenomenal" state (feeling textures) but the low mean rating for this item is very close to the neutral point and can be explained on sceptical grounds. ${ }^{91}$ It is also worth noting that the average of the means of the 32 items (4.76) was above and significantly different from the neutral point. ${ }^{92}$

\footnotetext{
${ }^{91}$ An explanation might be that the participants thought that only the entities with (non-physical) souls can feel and science can explain only physical phenomena.

${ }^{92} M=4.76, N=32, S D=0.53, t(31)=8.22, p<.001$.
} 
The second study from Gottlieb and Lombrozo (Study 2a) was similar to the first study. However, this time the dimension of introspective-phenomenology was broken down into three subdimensions: privileged first-person access ("Only an individual him- or herself can know that he or she is experiencing __ ; an outside observer might be able to guess but can't truly know”), introspection ("An individual having the experience can know he or she experiences __ through introspection: the examination of one's own internal feelings or reflection"), and subjective experience ("_ _ has a subjective experience associated with it-a 'feeling' of what it is like") (p. 124). No other dimensions were asked about. Note that only the sub-dimension of subjective experience is straightforwardly related to the prevalence of explanatory gap intuitions. As such, I analysed the means of 39 items that received ratings above the neutral point for this sub-dimension.

As in the first study, many items that are not typically considered as phenomenal (such as believing in god, logical reasoning and making moral judgements) received ratings for the sub-dimension of subjective experience that are above the neutral point. The same two phenomena as the previous study (believing in God and being able to feel and identify different types of textures with the sense of touch) received an average that is below the neutral point. (The means of these items3.84 and 3.92, respectively - were very close the neutral point). The average of the means for the possibility of scientific explanation of 39 items (4.99) was above and significantly different from the neutral point. ${ }^{93}$

To recapitulate, even if we assume (despite the contrary empirical data discussed in Chapters 4-6) that laypeople have a conception of phenomenal consciousness, then we would expect them to treat questions falling in the introspective-phenomenology dimensions as asking about phenomenal consciousness (as Chalmers assumes). This is not borne out by the data, however as the dimensions includes several items that are not typically considered as phenomenal. Further, and more importantly for present purposes, even if we accept that this language is understood in

${ }^{93} M=4.99, N=39, S D=0.60, t(38)=10.29, p<.001$. 
terms of phenomenal consciousness, the data nonetheless suggest that laypeople by and large lack explanatory gap intuitions.

\subsubsection{Diaz's Study}

Díaz (2021) conducted four studies to investigate whether laypeople have explanatory gap intuitions and metaphysical intuitions that phenomenal consciousness is not identical to physical processes. For the present context (the prevalence of explanatory gap intuitions), I will focus on the responses given for the questions about the explanatory gap. However, since the prevalence of metaphysical intuitions gives us important insights into the prevalence of explanatory gap intuitions, I will also discuss the responses to the metaphysical questions. Further, I will refer back to the mean responses to the metaphysical questions in Chapter 10 in relation to the meta-problem of consciousness.

In his first study (Study 1a), Diaz had participants read two stories. In one story, the feeling of pain was associated with the neural activity in the Dorsal Posterior region of the Insula (DPI). The participants were given both correlational information about the association and causal information. ${ }^{94}$ The other story similarly associates water with $\mathrm{H}_{2} \mathrm{O}$ by giving again both correlational and causal information. Each story was followed by two questions asking the participants to rate their agreement with the following statements that express the metaphysical and epistemic reduction theses, respectively: "The feeling of pain/the substance water is just neural activity in the DPI/chemical composition $\mathrm{H}_{2} \mathrm{O}$ " (Metaphysical Reduction question), and "The properties of pain/water are fully explained in terms of neural activity in the DPI/chemical composition $\mathrm{H}_{2} \mathrm{O}$ ” (Epistemic Reduction question) (p. 61). All the participants rated these statements on a scale from 1 ("strongly disagree") to 6 ("strongly agree").

\footnotetext{
${ }^{94}$ The correlational information was that the neuroscientists found that experiencing pain involves the neural activity in DPI while the causal information was that the specific localization of the neural activity in DPI determines the qualitative character of pain plus some examples to the relation between qualitative character of the pain and the location of the neural activity.
} 
The results were striking. $79.0 \%$ of the participants agreed (gave ratings higher than 3) with the metaphysical statements. That is, almost 4 in 5 participants agreed that the feeling of pain is just neural activity in the DPI, which strongly suggest that laypeople by and large lack the metaphysical intuition that so-called phenomenal experiences have some non-physical aspects. $81.0 \%$ of the participants agreed with the epistemic reduction statement, which clearly suggests that laypeople do not share explanatory gap intuitions, at least in the case of pain. As Diaz anticipates, however, one might claim that scientific findings regarding the relationship between pain and the neural activity in DPI may have nudged the participants towards thinking that pain is the neural activity in DPI and so can be explained in terms of neuroscience.

To test this possible objection, Diaz conducted Study $1 \mathrm{~b}$ which varied the amount of information that was given to the participants. One group received both correlational and causal information, as in Study1a, a second group was given only the correlational information, and the last group was presented no information. The results of the study showed that the amount of information did not influence participants' level of agreement with the metaphysical and epistemic reduction statement. The overwhelming majority of the participants still agreed with the metaphysical and epistemic reduction statements $(77.0 \%$ and $81.0 \%$, respectively). However, the results indicate that the participants were more likely to give higher ratings in the case of water than in the case of pain when they were not given the whole story (both correlational and causal pieces of information).

Study $2 \mathrm{a}$ further tested whether the preamble led participants to answer the questions affirmatively by modifying the vignette. The modified prompt included the information that the feeling pain is a subjective mental state while the neural activity is an objective state, and that the relationship between them has always been controversial and it is still controversial despite the findings listed. The participants were also asked to explain their disagreement with the metaphysical reduction statement.

The percentage of agreement with the metaphysical and epistemic reduction statements this time dramatically dropped to $57.0 \%$ and $47.0 \%$, respectively. However, this does not yet show that laypeople share the explanatory gap intuitions for three reasons. The first reason has already been 
noted by the author: Half the explanations given by those who disagreed with the metaphysical reduction statement mentioned problems about the science (such as the science of brain being in its infancy) while the other half justified their disagreement with pain being more than just neural activity. As Diaz notes, this suggests that around $25.0 \%$ of the participants were sceptical about the identity between the neural activity in DPI and feeling of pain (p. 66). On the plausible assumption that the respondents giving the negative answer to the epistemic question would mostly be the same participants giving the negative answer to the metaphysical question, this also suggests that around $25.0 \%$ of the participants were sceptical about the scientific explanation of feeling pain in terms of neural activity in DPI. Further, it is also possible that a sizable proportion in this percentage are sceptical because of some reasons that have nothing to do with phenomenal consciousness, such as the belief that feeling is indicative of the existence of a soul which cannot be fully explained in physical terms.

Second, even if when we ignore the justifications the participants gave for their scepticism about the metaphysical reduction statement, we cannot still say that a significant majority of participants rejected the epistemic reduction statement. Diaz notes that binomial tests indicated that $53.0 \%$ (the percentage of the sceptics about the epistemic reduction statement) was not significantly different from the neutral point of $50.0 \%$ (p. 65). In line with this, the mean response to the epistemic question was not significantly different from the neutral point.

As a third reason for why we cannot say that the majority of the participants in Study 2a share explanatory gap intuitions, remember that Study 1b suggested that the amount of scientific information about the association between feeling of pain and the neural activity in DPI is not a significant factor for the responses. If the amount of prompted information is not a significant factor, then the difference between the results of Study 2a and those of Study 1b's full information case (in which both correlational and causal information were included in the prompt) can be explained, at least for a considerable part, by another difference between the vignettes of Study $2 \mathrm{a}$ and Study 1b's full information case. Note that the former but not the latter underlines that there is an ongoing debate about whether the feeling of pain is identical to its neural correlate. This means that some participants were influenced by the ongoing debate among the authority figures. 
So, it is quite possible that some participants thought that the scientific evidence was not conclusive (because of the ongoing debate), and this led them to give neutral or negative responses. The mean response (4.08, which is above and significantly different from the neutral point) for Study $1 \mathrm{~b}$ 's "no information" case seems to support this possibility. Note that there was no reference to authority figures or any evidence in the vignette of Study 1b's "no information" case. That is, when no extra information that might nudge people towards thinking in a particular way was provided, the respondents were keen to endorse the epistemic reduction statement, which makes it plausible that talking about the ongoing debate in Study 2a nudged some participants towards thinking that the scientific evidence is not conclusive.

In light of these considerations, as Diaz notes, Study 2a suggests that a notable percentage of the scepticism is driven by consciousness-independent factors (p. 70). That is, there is a good reason to believe that a considerable proportion of sceptical responses are not attributable to the existence of explanatory gap intuitions. This is because, for a sceptical response to signal the existence of explanatory gap intuitions, it should be driven by consciousness-related factors.

To elucidate why some people are sceptical about the metaphysical and epistemic statement, Diaz conducted a follow-up study (Study $2 \mathrm{~b}$ ) that tested the role of consciousness-related and consciousness-independent factors in lay judgments about consciousness. The study used a similar vignette to that used in Study 2a. In addition to the epistemic and the metaphysical questions, the study asked participants to rate their agreement with the epistemic gap statement ("Neural activity in the DPI cannot explain what is like to feel pain") and the metaphysical gap statement ("The feeling of pain is a different thing than neural activity in the DPI"). Diaz found that these questions were consistently measuring the same underlying component or phenomenon, which he called "Problem Intuitions". The mean response for this common factor was 3.78 (on a 1-7 scale), which is below the neutral point of 4 , with $52.0 \%$ of responses being above the neutral point.

To discover what explains participants rejecting metaphysical and epistemic reduction statements, the study tested four possible factors. Two factors were science-related factors that Diaz called "Science Completeness" "Science can discover much more about the feeling of pain than what is 
mentioned in the text") and "Science Quality" ("The scientific findings presented in the text are an example of good science") (p. 69). The third factor was the level of reflection and measured by using the Cognitive Reflection Test 2, which includes four questions such as "If you are running a race and you pass the person in second place, what place are you in?" (Thomson \& Oppenheimer, 2016). The last factor was the level of inward-thinking as measured by the responses to some statements such as "Being in touch with emotions is essential" and "I can feel close to someone, even in moments of silence".

Diaz found that there was an inverse relationship between the ratings for Science Completeness and Science Quality and one's having problem intuitions, while the level of reflection and inward thinking had no significant effect (p. 70). That is, participants who considered science as complete and considered the given scientific evidence as an example of good science tended not to report problem intuitions whereas the others tended to display scepticism. These findings also suggest that, as Diaz concludes, participants' problem intuitions are driven by consciousness-independent factors (problems with the science) rather than problems with explaining pain in physical terms or finding a room for pain in the physical realm.

Overall, the mean responses given for the epistemic reduction statements in Diaz's studies suggest that the majority of laypeople do not have explanatory gap intuitions. Further, it should be noted that the scarcity of metaphysical intuitions is also suggestive of the lack of explanatory gap intuitions. This is because there is a strong relationship between A and B being identical and explanatory reduction of $\mathrm{A}$ to $\mathrm{B}$, at least for the majority of people. If one believes that pain is identical to neural activity in the brain, it is natural and rather plausible to think that one also believes that explaining the neural activity in the brain is explaining pain simply because one believes that the explanandum is the same in both cases. So, the non-prevalence of metaphysical intuitions suggested by Diaz's studies are also suggestive of non-prevalence of explanatory gap intuitions. 


\subsection{Concluding Remarks}

In this chapter I have argued that the existing studies in the literature suggest that Premise 2-2 is true: Epistemic gap intuitions are not prevalent. To do this I've shown that the existing studies indicate that two major types of epistemic gap intuitions (conceivability and explanatory gap intuitions) are not prevalent.

We have seen from the first study of Sytsma (2010) that laypeople mostly do not believe that inverted spectrum cases are possible. Peressini (2014) second study and the fifth study of Fischer and Sytsma (2021) suggest the same for zombie intuitions. Taken together, these studies lend credence to the idea that the folk do not share conceivability intuitions. Also, the first two studies in Gottlieb and Lombrozo (2018) and the studies presented in Díaz (2021) strongly support the scepticism about the prevalence of explanatory gap intuitions.

Overall, the existing studies in the literature suggest that epistemic gap intuitions are not widespread, and hence that Premise 2-2 is true. Yet, to further address the issue, the next chapter will discuss the results of two new studies that provide additional empirical evidence with regard to the prevalence of explanatory gap intuitions. 


\section{NEW STUDIES ON THE PREVALENCE OF EXPLANATORY GAP INTUITIONS}

To further establish Premise 2-2 (epistemic gap intuitions are not prevalent), in this chapter, I will discuss two new experiments investigating the prevalence of explanatory gap intuition. The first study includes metaphysical questions in addition to the questions about the explanatory gap (hereafter, "explanatory questions"), and the second study includes questions about whether the respondent can have a particular mental state/capability. Both studies provide substantial evidence with regard to the prevalence of the explanatory gap intuitions, as well as metaphysical intuitions about phenomenal consciousness in the case of the first study. These studies, for the most part, will be discussed in terms of explanatory gap intuitions, so, I will call them Explanatory Studies 1 and 2. I will discuss the results of these studies in $\$ 8.1$ and $\$ 8.2$, respectively. I conclude that both studies clearly suggest that laypeople by and large do not share explanatory gap intuition. Finally, I discuss some possible objections to this conclusion and reply to them in $§ 8.3$.

\subsection{Explanatory Study 1}

Explanatory Study 1 was designed to investigate metaphysical and explanatory intuitions by asking both metaphysical and explanatory questions. Explanatory questions are clearly relevant to the prevalence of explanatory gap intuitions. However, the relationship between the metaphysical questions and the prevalence of explanatory intuitions needs some explanation. The metaphysical questions are informative with regard to how widespread explanatory gap intuitions are because of the relationship between metaphysical reduction and explanatory reduction mentioned in §7.2.2. To repeat, there is a strong relationship between the belief that $\mathrm{A}$ is identical to $\mathrm{B}$, and the belief that explaining B fully explains A. That is, if one believes the former, then there is a good reason to assume that one also believes the latter. Even if this might not be true in all cases, it seems true for the questions at issue - for beliefs that humans are bodies and that explaining bodies can fully explain humans. So, we can assume that those who lack the metaphysical intuition- the intuition that phenomenal experiences have some non-physical feature (i.e. the phenomenal is over and beyond the physical) — by and large, also lack explanatory gap intuitions. I will first discuss the responses given for the metaphysical questions and will refer back to these results in Chapter 10 
in relation to the meta-problem of consciousness. Then I will discuss the answers given for the explanatory questions.

In the study, participants were asked two sets of questions, the first set being about humans and the second about robots. Each set included a metaphysical and an explanatory question. As such, each participant answered four questions in total, two being about humans and two being about robots.

The questions about robots were designed as control questions, serving to help assess whether scepticism about metaphysical and explanatory questions is related to phenomenal consciousness or not. In the case of metaphysical questions, if one thinks that neither humans nor robots are identical to their bodies, this suggests that the scepticism is due to reasons that are not related to phenomenal consciousness because humans, but not robots, are typically considered to be phenomenally conscious. On the other hand, if a participant reports that robots, but not humans, are identical to their bodies, it is possible that she shares the metaphysical intuition. Similarly, one may be considered as having the explanatory gap intuition only if she thinks that robots, but not humans, can be fully explained in physical terms. The questions about humans and the questions about robots were presented on separate pages and the order of the two pages was randomized to control for potential order effects.

The metaphysical question for humans read:

1. Are you identical to your body (where your body is taken to include your brain and how it is arranged), or is there something more to you beyond your body, or are you completely different from your body?

Participants were given the following four answer choices:

- I am identical to my body

- There is something more to me beyond my body

- I am completely different from my body 
- I don’t know

Participants selecting the last option were then asked which of the other three options they would select if forced to choose with the following preamble.

You answered that you don't know for the previous question. If you were nonetheless forced to choose, which of the other answers seems most likely to be correct to you?

The second question about humans was explanatory, with participants answering either "Yes" or "No":

2. Do you think that everything about humans could, in principle, eventually be fully explained by natural sciences (such as physics, biology, neuroscience, and the like) or do you think there is something about humans that could never be fully explained in scientific terms?

Participants were then asked to explain their answer, with the preamble "Please explain your answer to the previous question in the space below" for the respondents who answered the question affirmatively, and "What is it about humans that you think could never be fully explained in scientific terms?" for those who answered "No". The participants answering "No" were also asked the following question and options:

Why do you think humans could not be fully explained in scientific terms?

- There is something about humans that is beyond the reach of scientific explanation.

- While there is nothing about humans that is beyond the reach of scientific explanation, humans are too complex to be fully explained by science.

- While there is nothing about humans that is beyond the reach of scientific explanation, human scientists are too limited to ever produce such explanations.

The questions about the robots were similar. The metaphysical question about robots and the options were as follows: 
3. Is a robot identical to its body (where its body is taken to include its computer and how it is programmed) or is there something more to a robot beyond its body or is a robot completely different from its body?

- A robot is identical to its body

- There is something more to a robot beyond its body.

- A robot is completely different from its body.

- I don’t know.

As with the metaphysical question for humans, the participants selecting the last option were forced to choose one of the first three options. The second and explanatory question for robots was as follows:

4. Do you think that everything about robots could, in principle, eventually be fully explained by natural sciences (such as physics, biology, neuroscience, and the like) or do you think there is something about robots that could never be fully explained in scientific terms?

Participants were given only "Yes" and "No" options. The participants were then asked to explain their answers in the same way as the explanatory question for humans. The respondents answering "No" were also asked the following question and options:

Why do you think robots could not be fully explained in scientific terms?

- There is something about robots that is beyond the reach of scientific explanation.

- While there is nothing about robots that is beyond the reach of scientific explanation, robots are too complex to be fully explained by science.

- While there is nothing about robots that is beyond the reach of scientific explanation, human scientists are too limited to ever produce such explanations. 
Explanatory Studies 1 and 2 used the same recruitment method and restrictions as in the previous studies. 82 responses were included in the analysis. ${ }^{95}$ Overall, the results suggest that at most $40 \%$ of the participants seem to share the metaphysical intuition, and at most, around $28.5 \%$ of the participants might be considered as having the explanatory gap intuition. ${ }^{96}$ Despite these low figures, a majority do treat humans as having a further aspect and/or not being fully explicable in scientific terms. The key point for the purpose of the chapter, however, is that the scepticism does not appear to rest on intuitions about phenomenal consciousness.

Let's see first what the results suggest as to the prevalence of metaphysical intuition. Since robots are typically considered as non-phenomenally conscious entities, if one had the metaphysical intuition, one would tend not to think the same for robots. This is because the metaphysical intuition reflects that so-called phenomenal experiences have some non-physical aspects. Around $38.0 \%$ of the participants who were sceptical about the metaphysical statement about humans were also sceptical about robots being identical to their bodies. This suggests that a sizeable proportion of sceptics were sceptical for reasons that are not related to phenomenal consciousness. So, we should look at the participants who stated that robots, but not humans, are identical to their bodies for a proper estimation of the proportion of those having the metaphysical intuition.

\footnotetext{
95142 eligible participants failed or did not answer the attention check. Around $61.0 \%$ of the participants (included in the analysis) were native English-speakers, $67.0 \%$ were women, aged from 16 to 68 (average 26.6 years).

96 We found a significant, strong, negative correlation between Question 1-Forced and CRT 2 scores (but not CRT), and a significant, positive, but negligible correlation between Question 4 (Robot-explanatory) and CRT scores (but not CRT 2). So, it seems difficult to say that cognitive reflection scores played a significant role in participants' responses to the philosophical questions:

Question 1 (Human-metaphysical), CRT: $r=-0.07, p=0.57, N=64$; CRT 2: $r=0.2, p=0.1, N=66$.

Question 1-Forced, CRT: $r=-0.58, p=0.42, N=64$; CRT 2: $r=-0.91, p=0.03, N=66$.

Question 2 (Human-explanatory), CRT: $r=0.24, p=0.057, N=64$; CRT 2: $r=-0.23, p=0.059, N=66$.

Question 2-follow-up for sceptics, CRT: $r=-0.21, p=0.14, N=64$; CRT 2: $r=-0.24, p=0.086, N=66$.

Question 3 (Robot-metaphysical), CRT: $r=-0.11, p=0.38, N=64$; CRT 2: $r=0.074, p=0.55, N=66$.

Question 3-Forced, CRT: $r=-0.29, p=0.48, N=64$; CRT 2: $r=-0.29, p=0.45, N=66$.

Question 4 (Robot-explanatory), CRT: $r=0.27, p=0.033, N=64$; CRT 2: $r=0.21, p=0.091, N=66$.

Question 4-follow-up for sceptics, CRT: $r=0.22, p=0.51, N=64$; CRT 2: $r=-0.24, p=0.47, N=66$.
} 
$40.0 \%$ of the participants stated that robots, but not humans, are identical to their bodies. This proportion is significantly below $50 \% .{ }^{97}$ These findings suggest at least that it is not the case that the majority of people have metaphysical intuitions. Further, even this percentage overestimates the prevalence of metaphysical intuitions, given that one may think that robots, but not humans, are not identical to their bodies because of reasons that have nothing to do with phenomenal consciousness such as harbouring the belief that humans but not robots have souls. So, we have a good reason to infer that the majority of people lack metaphysical intuitions. ${ }^{98}$

What does this imply for the prevalence of explanatory gap intuitions? Because of the strong relationship between metaphysical and explanatory reduction mentioned before, if one had the intuition that humans are identical to their bodies, then one would tend to think that humans can be fully explained by natural sciences simply because they think that humans are just bodies. So, it seems that the answers to metaphysical questions already give us good reason to believe that

${ }^{97}$ One-tailed $\chi^{2}=2.74, d f=1, p=0.049$.

$\chi^{2}=2.74, d f=1, p=0.098$.

${ }_{98}$ The statistics for each metaphysical question were as follows: the distribution of responses for those who gave one of the first three responses to Question 1 versus on the follow-up question that forced the participants to choose one of the first three options was similar $\left(\chi^{2}=3.19, d f=2, p=0.20\right)$. As such, I will use the combined numbers in what follows. Initially, $29.2 \%$ participants answered that they were identical to their body, $51.2 \%$ that there was something more to them beyond their body, $9.75 \%$ that they were completely different from their body, and $9.75 \%$ that they didn't know. Including the responses to the forced-choice from those who did not know, 35.4\% answered that they were identical to their body compared to a significant majority giving one of the other two choices $64.6 \%$. No order effect was found for the combined results, meaning whether these questions (the initial and the follow-up question) were asked before or after the robot questions did not significantly matter $\left(\chi^{2}=1.57, d f=2, p=0.46\right)$. As with the metaphysical question about humans, no significant order effect was found for the metaphysical question about robots (asking whether a robot is identical to its body), although the effect was nearly significant $\left(\chi^{2}=5.21, d f=2, p=\right.$ 0.074). The participants answering the questions about humans first were more likely to answer that robots are identical to their bodies than the participants who answered the robot questions first (75.6\% versus $51.1 \%)$. The distribution of responses for those who gave one of the first three responses to this question versus on the follow-up question (that forced the participants to choose one of the first three options) was similar ( $\chi^{2}=1.33, d f=2, p=0.51$ ) so I will use the combined numbers in the rest of the discussion of the results for robots as well. In clear contrast to the responses given for the metaphysical question about humans, a significant majority (around 62.0\%) of the participants stated that robots are identical to their bodies while around $38.0 \%$ stated either that there is something more to a robot beyond its body or that a robot is completely different from its body $\left(\chi^{2}=4.40, d f=1, p=0.04\right)$. That is, participants were significantly more likely to answer that robots are identical to their bodies than humans (McNemar's chi-squared $=10.023, d f=1, p=0.0015)$. 
laypeople by and large lack explanatory gap intuitions. The answers to explanatory questions support this too, as will be explained in the rest of this section.

Looking at the answers given to the explanatory questions, if one had the explanatory gap intuition, we would expect participants to believe that robots (as non-phenomenally conscious entities) can be fully explained by the natural sciences but that humans (as phenomenally conscious entities) cannot be. Around $18 \%$ of the participants answered that neither humans nor robots can be fully explained. This suggests that a sizable proportion of sceptics about the explanatory statement about humans have reasons for their scepticism that have nothing to do with phenomenal consciousness. So, to properly estimate the prevalence of explanatory gap intuitions, we should first look at the proportion of the participants who answered that humans but not robots cannot be fully explained because there is something about them that is beyond the reach of scientific explanation. Further, we should look at the free-response answers of those who answered in this pattern to see if their scepticism relates to phenomenal consciousness.

The proportion who answered that humans but not robots cannot be fully explained was $42.7 \%$ $(35 / 82)$, which is below but not significantly different from $50.0 \% .{ }^{99}$ When we further look at the justifications given by these participants to explain their scepticism, six participants did not indicate any justification and only four out of 29 answers were found related to phenomenal consciousness even on a generous interpretation of the term. ${ }^{100}$ That is, even assuming that the six participants who did not explain their scepticism were sceptical for reasons related to phenomenal

${ }^{99} \chi^{2}=1.48, d f=1, p=0.22$.

10010 answers indicated that soul/spirit/paranormal events/after death cannot be explained by sciences while two answers stated that god and creation of man are beyond the reach of sciences. Four answers referred only to physical phenomena (body's operation, evolution, brain, energy/universe). I treated two answers mentioning consciousness and mind as being related to phenomenal consciousness, although it is not certain which aspect of the human mind is at issue and what sense of "consciousness" is meant. One answer refers to self-consciousness, so I did not treat it as being related to phenomenal consciousness. I also treated one answer mentioning feelings and another answer mentioning why people like some particular things which others don't as being related to phenomenal consciousness, although again, it is not clear the participants meant something phenomenal by "feelings" and "personal likings". There were also some responses mentioning thoughts, awareness of surroundings, human attitude, uniqueness and complexity of humans, and unconscious states. 
consciousness, around at most $28.5 \%$ of the respondents seem to have explanatory gap intuition. ${ }^{101}$ So, the answers to the explanatory questions clearly suggest that the majority of laypeople lack the explanatory gap intuition.

A worry about the study might be that the explanatory questions of the study were too general to properly investigate the lay intuitions about so-called phenomenal cases. That is, it is possible that people might be keener to accept the explanatory reduction statements about humans than to accept the explanatory reduction statements about specific phenomenal states. To put this possible objection differently, it is possible that if they were asked about specific phenomenal experiences, they would be keener to reject that these experiences can be fully explained by science while showing the tendency to endorse that non-phenomenal states can be fully explained by science. To test this objection, I conducted Explanatory Study 2.

\subsection{Explanatory Study 2}

The main purpose of Explanatory Study 2 was to test the prediction above. It included explanatory questions about 13 supposed phenomenal states. However, in order to use the study for more extended research in the future, we asked participants about non-phenomenal items as well. Also, for each item, we asked a non-explanatory question about whether the participant thinks she has

${ }^{101} \chi^{2}=20.50, d f=1, p<.001$.

Although the majority of the sceptics about the explanatory statement had reasons that are not related to phenomenal consciousness, it is worth noting some general statistics for each explanatory question. 74.4\% of participants answered that there is something about humans that could never be fully explained in scientific terms $\left(\chi^{2}=18.55, d f=1, p<\right.$ .001). No order effect was found for this question, i.e. whether the question is presented before or after the robot questions did not significantly matter $\left(\chi^{2}=1.06, d f=1, p=0.30\right) .68 .8 \%$ of the sceptics answered that there is something about humans that is beyond the reach of scientific explanation $\left(\chi^{2}=7.93, d f=1, p=0.0049\right)$. 24.6\% of the sceptics answered that humans are too complex to be fully explained by science while $6.6 \%$ answered that human scientists are too limited to ever produce such explanations.

As for the explanatory question for robots, a significant majority $(81.7 \%)$ answered that robots could be fully explained $\left(\chi^{2}=31.72, d f=1, p<.001\right)$. No order effect was seen for this question $\left(\chi^{2}=0.53, d f=1, p=0.47\right)$. Participants were significantly more likely to deny that humans could be fully explained than to deny that robots could be fully explained (McNemar's chi-squared $=37.50, d f=1, p<.001) .46 .67 \%$ of the sceptics about the explanatory statement for robots answered that robots could never be fully explained in scientific terms because there is something that is beyond the reach of scientific explanation, whereas $33.33 \%$ of the sceptics answered that robots are too complex and $20 \%$ of them answered that human scientists are too limited. 
or is capable of being in the given mental state just before the explanatory question. For the purpose of this chapter, however, I will report and discuss only the answers given for the explanatory questions about the phenomenal items.

First, participants were given the following preamble:

On each of the next set of pages is an item. For each item, we would like to know whether it is something that you have or are capable of and, if so, whether you think it is something that could, in principle, eventually be fully explained by natural sciences (such as physics, biology, neuroscience, and the like).

Each item was asked about on different pages, with each participant being asked about a subset of the items with the order randomized. On each page, the participants were given the following preamble:

On a scale of 1-7, one being totally disagree, seven being totally agree, how much do you agree with each of the following claims?

Each page included a non-explanatory statement asking whether the participant has or is capable of being in the given mental state (e.g. "I feel pain when I am injured") and an explanatory statement indicating that in principle science could eventually fully explain the given mental state (e.g., "In principle science could eventually fully explain pains"). ${ }^{102}$

102 The participants who gave the answers below the neutral point of $4(1,2$, or 3$)$ to the explanatory question were also asked a question that asked them to choose from three reasons for their scepticism. The follow-up question and its options were as follows:

Why do you think that this could not be fully explained in scientific terms?

- It is beyond the reach of scientific explanation.

- While it is not beyond the reach of scientific explanation, it is too complex to be fully explained by science.

- While it is not beyond the reach of scientific explanation, human scientists are too limited to ever explain it. Since, as will be seen below, the mean responses to the explanatory questions about so-called phenomenal states already clearly suggest that participants by and large did not have the explanatory gap intuition, I will not report nor discuss the answers to this follow-up question. 
If the objection to the previous study is accurate, the mean responses to the explanatory questions about so-called phenomenal states should be below and significantly different from the neutral point. However, the results clearly suggest the opposite.

I marked 13 items as "phenomenal" items on a generous interpretation of the term. These were as follows: pains and feelings of pain that followed the non-explanatory statement that "I feel pain when I am injured" ("pains-1" and "feelings of pain" hereafter); pains and experience of pain following "I experience pain when I am injured" ("pains-2" and "experience of pain" hereafter); colors following "I see colors" ("colors-1"); colors and experience of colors following "I experience colors" ("colors-2" and "experience of colors"); sounds, experience of sounds, emotions, moods, consciousness and being conscious.

514 responses were included in the analysis. ${ }^{103}$ The mean responses for all these items were above and significantly different from the neutral point. ${ }^{104}$ That is, as opposed to the expectation of the

1031733 eligible participants failed or did not answer the attention check. Around 57.0\% of the participants (included in the analysis) were native English-speakers, $62.0 \%$ were women (188 male, 319 female, and 7 non-binary), aged from 16 to 80 (average 26.54 years).

${ }^{104}$ Pains-1: $M=6.11, N=46, S D=1.3, t(45)=10.97, p<0.001 ; 82.6 \%(N=38)$ answering 5,6, or $7, \chi^{2}=19.57, d f$ $=1, p<0.001$.

Feelings of pain: $M=5.92, N=39, S D=1.31, t(38)=9.2, p<0.001 ; 79.5 \%(N=31)$ answering 5,6 , or $7, \chi^{2}=$ 13.56, $d f=1, p<0.001$.

Pains-2: $M=5.75, N=65, S D=1.3, t(64)=10.88, p<0.001 ; 81.5 \%(N=53)$ answering 5,6, or $7, \chi^{2}=25.86, d f=$ $1, p<0.001$.

Experience of pain: $M=5.89, N=57, S D=1.63, t(56)=8.76, p<0.001 ; 77.2 \%(N=44)$ answering 5,6, or $7, \chi^{2}=$ 16.86, $d f=1, p<0.001$.

Colors-1: $M=5.7, N=47, S D=1.67, t(46)=7, p<0.001 ; 78.7 \%(N=37)$ answering 5,6, or $7, \chi^{2}=15.51, d f=1$, $p<0.001$.

Colors-2: $M=5.27, N=49, S D=1.48, t(48)=5.97, p<0.001 ; 65.3 \%(N=32)$ answering 5,6, or $7, \chi^{2}=4.59, d f=$ $1, p=0.032$.

Experience of colors: $M=5.07, N=58, S D=1.81, t(57)=4.49, p<0.001 ; 62.1 \%(N=36)$ answering 5,6 , or $7, \chi^{2}$ $=3.38, d f=1, p=0.066$.

Sounds: $M=5.81, N=52, S D=1.25, t(51)=10.4, p<0.001 ; 78.8 \%(N=41)$ answering 5,6, or $7, \chi^{2}=17.31, d f=$ $1, p<0.001$.

Experience of sounds: $M=5.41, N=58, S D=1.69, t(57)=6.39, p<0.001 ; 63.8 \%(N=37)$ answering 5,6 , or $7, \chi^{2}$ $=4.41, d f=1, p=0.036$.

Emotions: $M=5.06, N=47, S D=2, t(46)=3.64, p<0.001 ; 68.1 \%(N=32)$ answering 5,6, or $7, \chi^{2}=6.15, d f=1$, $p=0.013$. 
objector, participants found the possibility of a full scientific explanation of pains, colors, sounds (as well as feelings and/or experiences of them), emotion, moods and consciousness realistic. As with Gottlieb and Lombrozo (2018), Díaz (2021) and Explanatory Study 1, the study strongly suggests that laypeople by and large do not share the explanatory gap intuition. ${ }^{105}$

However, it might be argued that one's endorsing the possibility of scientific explanation of phenomenal states does not necessarily mean that one does not have explanatory gap intuitions, and thus that these studies do not provide us with reliable data to investigate the prevalence of explanatory gap intuitions. I will reply to two objections along these lines in the next section.

\subsection{Do the Studies Really Suggest that Explanatory Gap Intuitions are not Prevalent?}

There might be two reasons to think that one's positive answers to the questions about explanatory statements about supposed phenomenal states do not mean that one does not share the explanatory

Moods: $M=5.02, N=46, S D=1.61, t(45)=4.3, p<0.001 ; 54.3 \%(N=25)$ answering 5,6, or $7, \chi^{2}=0.35, d f=1$, $p=0.56$.

Consciousness: $M=5.38, N=65, S D=1.51, t(64)=7.41, p<0.001 ; 69.2 \%(N=45)$ answering 5,6 , or $7, \chi^{2}=9.62$, $d f=1, p=0.0019$.

Being conscious: $M=4.98, N=59, S D=1.69, t(58)=4.48, p<0.001 ; 55.9 \%(N=33)$ answering 5,6 , or $7, \chi^{2}=$ $0.83, d f=1, p=0.36$.

${ }^{105}$ We found a significant, positive, but small correlation between "Colors-1" and CRT scores (but not CRT 2). Also, there was a significant, negative, but small correlation between "Experience of colors" and the CRT scores, as well as a significant, negative, but negligible correlation between the same item and the CRT 2 scores. So, it can be safely said that the cognitive reflections scores were not a significant factor in participants' responses to the philosophical questions:

Pains-1, CRT: $r=-0.32, p=0.076, N=356$; CRT 2: $r=-0.08, p=0.66, N=389$.

Feelings of pain, CRT: $r=-0.02, p=0.92, N=356$; CRT 2: $r=-0.2, p=0.32, N=389$.

Pains-2, CRT: $r=-0.01, p=0.97, N=356$; CRT 2: $r=0.15, p=0.3, N=389$.

Experience of pain, CRT: $r=-0.09, p=0.58, N=356$; CRT 2: $r=0.0039, p=0.98, N=389$.

Colors-1, CRT: $r=0.19, p=0.3, N=356$; CRT 2: $r=0.35, p=0.047, N=389$.

Colors-2, CRT: $r=0.1, p=0.59, N=356$; CRT 2: $r=-0.06, p=0.72, N=389$.

Experience of colors, CRT: $r=-0.35, p=0.021, N=356$; CRT 2: $r=-0.29, p=0.049, N=389$.

Sounds, CRT: $r=0.15, p=0.39, N=356$; CRT 2: $r=0.095, p=0.56, N=389$.

Experience of sounds, CRT: $r=-0.28, p=0.086, N=356$; CRT 2: $r=-0.17, p=0.27, N=389$.

Emotions, CRT: $r=0.046, p=0.81, N=356$; CRT 2: $r=-0.23, p=0.2, N=389$.

Moods, CRT: $r=0.059, p=0.74, N=356$; CRT 2: $r=-0.06, p=0.74, N=389$.

Consciousness, CRT: $r=0.13, p=0.38, N=356$; CRT 2: $r=0.2, p=0.15, N=389$.

Being conscious, CRT: $r=0.24, p=0.16, N=356$; CRT $2: r=-0.06, p=0.7, N=389$. 
gap intuition, based on the possible ambiguity of the terms "science" and "explanation" respectively.

The first objection is that the term "Science" can be read by some people so generously that one can think, for example, that science in a very broad sense can explain our painful experiences but this explanation would require more than biological, physical or neural explanation. Chalmers (2020, p. 13) claims, for example, that even a property dualist, who basically believes that there are non-physical properties in the world, can accept that science can in principle explain phenomenal states in a generous sense of "science". So, Chalmers thinks that asking people to rate their agreement with scientific explanation statements is not a good choice to investigate the prevalence of explanatory gap intuitions and finds Gottlieb and Lombrozo (2018) problematic for this reason. He suggests that the experimenter ask whether neuroscience (instead of science) can explain a mental state.

Admittedly, the notion of science might be treated narrowly or broadly by different people, and in the latter case, people would be more likely to think that science in principle can fully explain phenomenal states even if they tend to think that this explanation would be beyond the limits of the natural sciences. For this reason, I believe that the explanatory statement in Gottlieb and Lombrozo's study (“Science could one day fully explain” such-and-such mental capability or state) is not the perfect choice to investigate the prevalence of explanatory gap intuitions. This is because there are many people who consider some non-scientific intellectual efforts (such as fringe science, far-fetched interpretations of quantum physics that go well beyond the scientific evidence, parapsychology, and even astrology) scientific. However, I also believe that laypeople mostly would read "science" in the sense of brain sciences when they think about whether "science" can fully explain mental states. So, Gottlieb and Lombrazo's study cannot be dismissed easily.

But in any case, the objection does not apply to Diaz's study and Explanatory Studies 1 and 2. This is because the term "science" in Diaz's study and Explanatory Studies 1 and 2 refers to a narrow sense of the term with clear references to neuroscience. Remember that the epistemic reduction statement in the studies of Díaz (2021) was "The properties of pain are fully explained 
in terms of neural activity in the DPI". And Explanatory Study 1 and 2, used the questions that refer to "natural sciences (such as physics, biology, neuroscience, and the like)" rather than only "science".

The second objection is that one might deploy a sense of "explanation" according to which phenomenal states can be "explained" in a specific or too generous sense of the term but one might still tend to think that the phenomenal state in question (or phenomenal consciousness in general) still remains unexplained in another sense. Chalmers states this worry as follows: "But still different notions of explanation may be deployed in answering it. For example, even a dualist could say yes if they think causal explanation is at issue and neural processes cause consciousness" (Chalmers, 2020, p. 241).

I agree that philosophers who believe in the explanatory gap might still tend to endorse that science can explain everything in principle by appealing to different senses of "explanation". But I do not think that laypeople would think about the explanatory/reduction statements in a way that makes a distinction between different senses of the term. Further, both Diaz's studies and Explanatory Studies 1 and 2 asked the participants whether science can "fully explain" the target phenomenon which includes causal explanation but is not limited to it. So, as with the first objection, the objection seems not to exactly hit these studies.

Based on the considerations above, these two objections relying on the possible ambiguity in the terms "science" and "explanation" do not cast considerable doubt on our conclusion that the explanatory studies discussed in this and Chapter 7 suggest that laypeople by and large do not share explanatory gap intuitions. Further, even if some participants that share the explanatory gap intuition gave positive answers to the explanatory statements because of their generous or special reading of "science" or "explanation", it would be far-fetched to claim that this alone accounts for the clear majority endorsing these statements (and thus the results of the studies are not reliable to investigate the prevalence of the explanatory gap intuitions). One obvious reason to think that why such participants were not the majority is that both Diaz's studies and Explanatory Study 1 suggest that laypeople tend to consider phenomenal states as being identical brain states. Remember that 
Study 1a and 1b in Diaz's work clearly suggest that the majority of laypeople believes that the feeling of pain is just neural activity. Remember also that Explanatory Study 1 suggests that the majority of laypeople do not share the metaphysical intuition reflecting that phenomenal experiences have some non-physical features. That is, these two studies support the idea that socalled phenomenal states are just brain states for the majority of people. This gives us a solid reason to believe that the participants in the studies discussed endorsed the explanatory statements mainly because of their reasoning that science in principle can explain any physical state thus it can explain "phenomenal" states as well because they are just physical states.

\subsection{Concluding Remarks}

The chapter introduced two new studies to further investigate the prevalence of explanatory gap intuitions. The results confirmed the existing studies discussed in the previous chapter: a clear majority of laypeople have no issue with the possibility of explaining so-called phenomenal states.

Remember that Chapters 4-6 clearly suggested that existence intuitions (experiential property and common aspect intuitions) are not prevalent. The findings of this and the previous chapter give us good reason to think that two main sorts of epistemic gap intuitions (conceivability and explanatory gap intuitions) are not prevalent either. Together these provide strong support for Premise 2 of the General Argument, indicating that phenomenal intuitions are not prevalent. I now proceed to defend Premises 2 and 3 against possible objections and conclude the General Argument.

\section{CONCLUDING THE GENERAL ARGUMENT}

This chapter aims to deal with several outstanding concerns regarding Premises 2 and 3 of the General Argument and to show that the conclusion straightforwardly holds if the premises are true. As a reminder, the General Argument is:

(Premise 1): Phenomenal theses (phenomenal realism and the epistemic gap thesis) are primarily defended by appealing to phenomenal intuitions. 
(Premise 2): Phenomenal intuitions are not prevalent.

(Premise 3): Appeals to phenomenal intuitions that are not prevalent do not provide compelling evidence for phenomenal theses.

Conclusion: The primary way of defending phenomenal theses fails.

Chapters 2-3 aimed to show that Premise 1 is true based on abundant textual evidence clearly indicating not only that the main way of defending phenomenal theses is by appealing to phenomenal intuitions but also that prominent proponents of phenomenal theses, such as Chalmers, happily admit that intuitions are of central importance to these theses. For this reason, I believe that Premise 1 is the least controversial premise of the argument. So, the more challenging task is to defend Premises 2 and 3. §9.1 will defend Premise 2 while §9.2, §9.3 and §9.4 will collectively defend Premise 3. Finally, $\$ 9.5$ will argue that since the General Argument is valid, and the premises of the argument are plausible, the conclusion of the argument is also plausible.

\subsection{Defence of Premise 2}

Chapters 4-8 discussed and presented a wide range of empirical studies supporting Premise 2. In these chapters, I have also replied to some possible and existing objections to the empirical studies and my interpretation of the results. Based on the findings discussed in these chapters, I concluded that neither existence intuitions (experiential property and common aspect intuitions) nor epistemic gap intuitions (conceivability and explanatory gap intuitions) are prevalent. This conclusion rests on the idea that the empirical studies provide us with a good measure of the prevalence of phenomenal intuitions.

Against this idea, and thus Premise 2, it might be argued that one's responses to a question in a survey might not reflect the intuitions the question was intended to assess. So, the critic claims that the empirical studies discussed above solicit participants' reports, not necessarily their intuitions. And, thus, we cannot conclude from these results that most of the participants lack phenomenal intuitions. 
Why would people having phenomenal intuitions respond to the surveys in ways that don't reflect their intuitions? A straightforward answer is that some factors ("blocking factors", hereafter) might block reporting their phenomenal intuitions, which is why I will call this the "Blocking Factors Objection". The objection asserts that we should not expect that phenomenal intuitions will necessarily lead to responses suggestive of phenomenal theses because those intuitions might be blocked in one way or another, so the results of the studies discussed might be misleading. Another answer might be that phenomenal intuitions are latent, such that people's immediate responses to questions will be unlikely to reveal them. ${ }^{106}$ And if this is the case, the results of the studies discussed would not be supportive of Premise 2. I will call this the "Latent Intuitions Objection". In the rest of this section, I will discuss the Blocking Factors Objection and Latent Intuitions Objection.

\subsubsection{Blocking Factors Objection}

With the Blocking Factors Objection in mind, it is not difficult to see that the idea that the empirical studies provide us with a good measure of the prevalence of phenomenal intuitions rests on the following assumption:

If one had a phenomenal intuition, she would most probably report what the intuition reflects in the surveys, at least when the factors that might plausibly prevent reporting in accordance with her intuition ("blocking factors" hereafter) are controlled for.

The assumption holds that further controlling for blocking factors would not notably alter the prevalence of phenomenal intuitions. In other words, it contends that there is no reason to think that phenomenal intuitions are prevalent but that a notable number of participants having these intuitions did not report them in the studies discussed because of blocking factors. This section will defend Premise 2 by replying to the main objection to the assumption above.

106 Thanks for Simon Keller for raising this possibility. 
What could such blocking factors be? For one thing, the objector can claim that there might be confounding effects (order effects, framing effects, and so on) in the empirical studies that lead participants to give responses that do not reflect their intuitions. To avoid being ad hoc, the objector should explain which experiments discussed might be susceptible to which confounding effects. Further, the objector should also explain why we should expect a radical change in results when these effects are eliminated. After all, in the abstract, it is reasonable to expect that confounding effects could affect both the majority of participants who seem not to have phenomenal intuitions and the minority who seem to have them.

In addition to confounding effects that any type of experiment might suffer from, the objector can also claim that phenomenal intuitions might be blocked by some beliefs that conflict with them. Chalmers gives an example: "an inclination towards dualism might be outweighed by reasons to accept physicalism" Chalmers (2018, p. 14). As another example, one's respect for the explanatory power of the natural sciences might block one's reporting explanatory gap intuitions. It is true that there might be some factors that outweigh one's intuitions and thus lead to judgments that diverge from participants' intuitions. However, there might also be background beliefs that lead people to respond as if they have phenomenal intuitions. For example, to reverse the possibility Chalmers mentions, anti-realist intuitions (or intuitions that go against the epistemic gap) might be outweighed by reasons to accept dualism. Dennett (1991, p. 372) gives a more specific example: Since people think that science tells us that there is no color in the outside world, they may tend to think that colors must be located in our minds. That is, one may state that colors belong to mental states not because she has the experiential property intuition but because she holds that there is no room for colors in the external world and thus reasons that colors must be in our minds. So, the objector needs to explain why we should expect a radical change in results when there is no conflicting belief that prevents phenomenal intuitions from being reported.

One might also worry that some cultures might be less keen to report phenomenal intuitions because people raised in those cultures might be more vulnerable to blocking factors, and thus a proper investigation of the prevalence of phenomenal intuitions needs to be cross-cultural. Thus, SM might be criticized for only exploring the intuitions of people from the USA. However, there 
is no reason to think that the results of cross-cultural studies would be more supportive of the idea that phenomenal intuitions are prevalent. After all, as noted, the cross-cultural studies that followed up on SM's studies, such as Sytsma and Ozdemir (2019), suggested the same overall pattern of findings as in SM. Further, all the new studies I've reported except Ambiguity Study 4 (Ambiguity Studies 1-3, Dimensions Studies 1-2, and Explanatory Studies 1-2) weren't restricted to participants from the US and involved participants who hailed from across the world.

I noted above that the advocate of the Blocking Factors Objection owes us an explanation of why we should expect that eliminating potential blocking factors would make a big difference in the overall findings that the majority of people seem to lack phenomenal intuitions, considering that there is no reason to think that these factors affect the majority more than the minority. In reply, one can claim that the responses of one specific group in the minority are less susceptible to blocking factors and thus provide more reliable data for the purpose of assessing the prevalence of phenomenal intuitions - philosophers! The underlying idea - a version of the so-called "Expertise Objection" (Hales, 2009; Ludwig, 2007; Sorensen, 2014) to the negative program in experimental philosophy more generally — is that because of their training in logic, occupational involvement in conceptual analysis, familiarity with dealing with thought experiments, and so on, philosophers are more likely to state their intuitions correctly.

Thus, the critic may claim that the intuitions of philosophers are less likely to be blocked than the intuitions of laypeople. For example, philosophers are less likely to suffer from "scientific" bias, which would lead people with high regard for the explanatory power of the natural sciences to tend to answer the explanatory questions affirmatively, and thus philosophers would be more likely to report their explanatory gap intuitions. The objector might also claim that philosophers are less vulnerable to other effects - such as framing effects, order effects and so on - that might prevent their phenomenal intuitions from being reported. And, the objector goes on, we know that the majority of philosophers gave responses suggesting that they have existence intuitions in SM and that they mostly believe that philosophical zombies are conceivable (Bourget \& Chalmers, 2014, p. 477). Note that if the objection is accurate, laypeople would not be the best sample to use to examine the prevalence of phenomenal intuitions, depending on how prone to potential blocking 
factors one takes the studies to be. This would then at least cast doubt on the conclusions we could draw from the studies discussed above. As a matter of fact, if the expertise objection is true, the opposite of Premise 2 would be supported because the right sample would be philosophers and there is reason to believe that the majority of philosophers have phenomenal intuitions.

However, there are two main reasons to discount the Expertise Objection here. First, when it comes to examining the prevalence of phenomenal intuitions, non-philosophers would seem to be better candidates because they are the ones who judge their subjective experiences without employing philosophical concepts about phenomenal consciousness (such as "phenomenal" and "qualitative") and without considering philosophical theories on phenomenal consciousness they learnt in philosophy classes. Note that the studies we discussed generally asked the participants about ordinary concepts such as colors, pain, and so on, which Chalmers labels as "specific phenomenal concepts" and thinks are enough for one to have phenomenal intuitions (Chalmers, 2018, 2020). And it is reasonable to think that laypeople are better intuiters when it comes to evaluating some questions that involve only ordinary concepts because trained philosophers are under the influence of the relevant philosophical theories (Machery, Mallon, Nichols, \& Stich, 2004, p. B9), which may distort their intuitions about them. The basic worry here is that when it comes to philosophers, we have reason to doubt that their judgments reflect their pure intuitions rather than their training and theoretical commitments.

Second, empirical research indicates that philosophers are not immune to order and framing effects. To give but a few examples, Schwitzgebel and Cushman (2012) found that philosophers are also susceptible to order effects in their judgements about various moral scenarios. And Tobia, Buckwalter, and Stich (2013) found that they are also susceptible to a particular type of framing effect (the actor-observer bias).

To summarize, I've argued that there is no reason to think that eliminating blocking factors would make a considerable difference in the findings surveyed, no reason to think that the findings are specific to Americans, and no reason to think that professional philosophers would provide a better 
sample for assessing phenomenal theses. These considerations support the assumption for Premise 2. Now, I will discuss the Latent Intuitions Objection.

\subsubsection{Latent Intuitions Objection}

A critic might admit that there is no reason to think that eliminating all the blocking factors would radically change the results of the studies discussed, but still insist that the responses of the participants do not necessarily reflect their intuitions because it is possible that they may have (latent) phenomenal intuitions that do not reveal themselves in these types of studies. That is, phenomenal intuitions may need some revealing conditions under which a belief that supports a phenomenal thesis seems true to one who has these intuitions. If these conditions are not present, phenomenal intuitions remain latent. The objection claims that these conditions might not be present in surveys. So, a person with latent phenomenal intuitions might still give responses that are inconsistent with phenomenal theses.

We cannot ignore this possibility. Yet, it would be ad hoc to dismiss the empirical data discussed just because of this possibility unless the objector provides some plausible reasons to think that this is the case. In the absence of such reasons, it is reasonable to assume that the responses do indeed generally reflect people's intuitions. No one would complain about using more sensitive, deeper-level psychological or neuroscientific tools to detect phenomenal intuitions (latent or not) if possible, but considering that we do not have such tools yet, it should be admitted that responses to survey questions provide our best current data. After all, asking laypeople questions is the most common way (if not the only way) to study lay intuitions in experimental philosophy and psychology.

Furthermore, there seems no reason to think that phenomenal intuitions are latent and, thereby, to think that Chalmers $(2018$, p. 13) is wrong when he says that "it is highly plausible that versions of many of these intuitions can be teased out of ordinary subjects" (p. 13). We all have some intuitions that straightforwardly produce beliefs we are aware of on different subject matters. That 
is, we have intuitions that are far from being latent. ${ }^{107}$ I simply do not see any reason to think that phenomenal intuitions are exceptional. There are obviously some ordinary people around us and among the participants in the studies discussed who seem to have phenomenal intuitions. There are people who believe, for example, that even a complete set of physical facts would leave the question of why we see red rather than green when we look at blood unanswered when they think about a relevant question for the first time in their life. As long as they have this sceptical belief without any underlying reason (based on perception, testimony, inference, etc.), then they have explanatory gap intuitions. And these intuitions straightforwardly produce corresponding beliefs or responses people are aware of, and thus are not latent at all. And, if some people have non-latent explanatory gap intuitions, as it seems, there seems no reason to think that other phenomenal intuitions are any different. At least, the objector owes us an explanation of why (most) phenomenal intuitions are exceptional.

Also, the possibility of phenomenal intuitions being latent raises a crucial question: If phenomenal intuitions are latent, would it still be possible to plausibly claim that the existence of phenomenal consciousness and epistemic gap is intuitively obvious? In other words, if phenomenal intuitions are latent, would it still possible to appeal to these intuitions in defending phenomenal theses? The answer seems to depend on whether these intuitions can ever be revealed to us (and thus cease to be latent) or not. If they will always remain latent no matter what, it would be difficult to claim that phenomenal consciousness (or epistemic gap) is intuitively obvious to us. After all, the motivation behind the Latent Intuitions Objection is that people may have beliefs that are inconsistent with phenomenal theses despite their (latent) phenomenal intuitions. And if one had such beliefs, the existence of phenomenal realism or epistemic gap would not be obvious at all for that person. So, we would not plausibly claim that the existence of phenomenal consciousness or epistemic gap is intuitively obvious. As a matter of fact, in this case, we would have more reasons

\footnotetext{
${ }^{107}$ As noted before, I have the intuition that switching doors would not matter regarding Monty Hall problem. I can understand why my intuition is wrong from the statistical explanation; however, whenever I think about this problem, my intuition forms an immediate belief reflecting that switching doors would not matter at all! I once even developed a computer program that simulates the problem. Even after running the simulation, which of course showed that statistical explanation rather than my intuition is correct, there was (and is) still no gap between my immediate reaction to the problem and my intuition.
} 
to suspect that philosophers do not actually appeal to their intuitions but inadvertently mistake their non-intuition-based beliefs for intuitions. After all, if phenomenal intuitions will always be latent in any case, they would always be latent for philosophers as well. Perhaps they would have some beliefs due to their intuitions, but they would not be aware of the source of their beliefs. In sum, if phenomenal intuitions are destined to be latent, the latency of them would be an objection to the intuition-based way of defending phenomenal theses rather than the General Argument.

Alternatively, one might think that phenomenal intuitions can be revealed to us (and thus cease to be latent) when some special conditions are satisfied. I can imagine three conditions that the objector can consider.

First, perhaps latent phenomenal intuitions can be revealed if we think about so-called phenomenal experiences carefully enough. That is, devoting a sufficient amount of attention would reveal phenomenal intuitions. After all, the objector may claim, if people thought about supposed phenomenal experiences carefully enough, their latent intuitions probably would be revealed to them. Based on this possibility, the objector can claim that this explains why most philosophers' phenomenal intuitions are not latent and relatedly why they are in a position to appeal to phenomenal intuitions to defend phenomenal theses. If this is the case, the objector would predict that more attentive respondents in the surveys discussed would be more likely to answer in a way that is consistent with their phenomenal intuitions. A similar idea (Reflection Objection) will be discussed in $§ 9.2 .2$ in more detail. To briefly prefigure that discussion, I argue that there is no easy way to determine which participants in the studies were more attentive or considerate while answering the questions. However, it is reasonable to assume that the participants who successfully answered cognitive reflection questions were more likely to be attentive or careful than others. And the correlation tests indicate that answers did not importantly vary with reflectivity. So, this first possibility does not seem realistic.

Second, perhaps for our phenomenal intuitions to cease to be latent, we need to be involved in relevant philosophical discussions. At least, the objector goes on, we need to hear the relevant arguments/ideas. For example, one might answer in the survey that science can explain everything 
including so-called phenomenal experiences but after reading about the explanatory gap idea, the same person might realize that he has always been disposed to think that there is an explanatory gap but that he was not aware of this. Now, it seems obvious to that person that there is an explanatory gap. However, in this story, the source of the person's new belief is not clear. Such a person might be under the influence of some ideas that they read, or not. In the former case, it would be difficult to claim that the new belief is due to one's intuitions. This is because, if one is under the influence of some new ideas that they read, then one has some explicit reasons supporting the new belief. But perhaps, the objector can point out, the ideas one reads might trigger the revelation of phenomenal intuitions without requiring one to be under the influence of those ideas. I can imagine two explanations of such a revelation: The ideas in question might cause one to think about a philosophical question about phenomenal consciousness for the first time in one's life. But note that if just thinking about a relevant philosophical question suffices to reveal a latent phenomenal intuitions, then we would expect that the philosophical questions in the surveys might also help reveal one's phenomenal intuitions. People who have latent explanatory gap intuitions, for example, would probably answer negatively when they think about whether feeling pain is "something that could, in principle, eventually be fully explained by natural sciences (such as physics, biology, neuroscience, and the like)" (the statement that I used in Explanatory Study 2). Against this reasoning, one might object that the questions in the surveys do not provide the respondents with enough context for them to reveal their phenomenal intuitions. However, it should be admitted that the more ideas provided to the respondents would invite more doubts on whether the respondents appeal to their pure intuitions.

The other explanation of one's revealing phenomenal intuitions after reading relevant ideas without being under the influence of those ideas is that the relevant ideas might make one carefully think about the nature of so-called phenomenal experiences. For example, one can carefully think about Levine's idea that any amount of physical explanation seems unable to explain why we see red rather than yellow when we look at blood. One can be completely neutral when one reads about this idea, but the idea might nonetheless lead them to think carefully enough to reveal their intuition that seeing colors cannot be explained in physical terms. However, note that this amounts to the same idea I've already discussed above: Carefully thinking about so-called phenomenal 
experiences reveal latent phenomenal intuition. To repeat briefly, there seems no reason to think that more reflective people are more likely to have beliefs that are consistent with phenomenal experiences. So carefully thinking does not seem to help us reveal our latent phenomenal intuitions, if we have them.

Lastly, the objector can claim that for phenomenal intuitions to be revealed, one should be inwardthinking. That is people, in Chalmers' terms, who can "easily attend to their mental lives" (Chalmers, 2020, p. 251) are more likely to uncover their latent intuitions. Again, I will discuss a similar idea in 9.2.1. To prefigure briefly, as noted in $\$ 7.2 .2$, Díaz (2021) reported that there is no significant correlation between the level of inward-thinkingness and one's having metaphysical and explanatory gap intuitions. So, inward-thinkingness seems not very helpful for us to reveal our latent phenomenal intuitions, if we have them.

In light of the considerations above about Blocking Factors and Latent Intuitions Objections, we have reason to take the studies discussed to provide us with an approximate estimation of the prevalence of phenomenal intuitions. And if this is the case, then Premise 2 of the General Argument is plausible because the studies discussed clearly indicate that phenomenal intuitions are far from being widespread.

\subsection{Defence of the Assumptions behind Premise 3}

We have seen that the studies discussed provide us with what seem to be reliable data for assessing the prevalence of phenomenal intuitions, and thus that there seems to be little reason to doubt Premise 2 of the General Argument. In this section, I will defend the assumptions behind Premise 3 - that appeals to phenomenal intuitions that are not prevalent do not provide compelling evidence for phenomenal theses. And, then, in the next two sections, I will argue that if these assumptions are plausible, then Premise 3 is also plausible.

Note that Chapters 4-8, which supported Premise 2, discussed empirical data about the prevalence of phenomenal intuitions without looking at participants' different introspective skills, conceptual repertoire, or their different levels of reflectiveness. In this sense, the sample of the studies was 
"general" and thus provided us with general data. Note also that Premise 3 rests on the main assumption that this general data are relevant to assessing phenomenal theses. That is, we can argue for Premise 3 based on this general data. To be more specific, we can argue that philosophers' appeals to their phenomenal intuitions do not provide compelling evidence for phenomenal theses considering that these intuitions are not prevalent in the general sample. Admittedly, this assumption is controversial because one can argue that what matters is not the general prevalence of phenomenal intuitions but the prevalence of these intuitions in some special groups. And if these intuitions are prevalent in these groups, then philosophers' appeals to phenomenal intuitions still provide compelling evidence for phenomenal theses even if their intuitions are not prevalent in the general sample. So, a proper defence of Premise 3 firstly requires a defence of the main assumption above.

This main assumption rests on the following two underlying assumptions:

Assumption 1: If phenomenal consciousness is pretheoretically obvious, no special introspective skills or new concepts are required for a person to have phenomenal intuitions.

Assumption 2: The level of reflectiveness is not a significant factor in having phenomenal intuitions.

Assumption 1 relies on the idea that if phenomenal consciousness is pretheoretically obvious, then the existence of phenomenal consciousness and the epistemic gap is something that should be easily recognized by laypeople. And if so, one should not need any special introspective skill or new concepts about phenomenal consciousness to have phenomenal intuitions. To make this more concrete, if phenomenal consciousness is pretheoretically obvious, one does not need to learn any new concept about phenomenal consciousness or to have any special introspective skills to have, for example, the intuition that feeling pain cannot be fully explained in physical terms even in principle. Following the same example, Assumption 2 holds that it is not the case that more reflective people who carefully think about feeling pain would be significantly more likely to have this intuition compared to less reflective people. I will defend Assumption 1 and 2 by showing that the objections to them fail in $\S 9.2 .1$ and $\$ 9.2 .2$, respectively. 


\subsubsection{Defence of Assumption 1}

Against Assumption 1, one could contend that a person needs to have some introspective skills or learn new concepts to have phenomenal intuitions, and that this is compatible with phenomenal consciousness being pretheoretically obvious. After all, the objector claims, one can see that something is obvious after gaining some skills or new concepts. So, the objection goes, what matters for assessing phenomenal theses is not the prevalence of phenomenal intuitions among laypeople but among those people who have the required introspective skills or conceptual repertoire. Call this the "Requirements Objection". I will discuss two versions of the Requirement Objection in the rest of this sub-section.

\section{Are Special Introspective Skills Required to Have Phenomenal Intuitions?}

Let's think first about introspective skills that may be required for phenomenal intuitions. It might be argued that although the existence of phenomenal consciousness is phenomenologically obvious, phenomenal intuitions require one to introspect on one's phenomenal experiences more carefully or skilfully than might occur by default. And if this is the case, the studies discussed above are too inclusive to properly assess phenomenal theses.

The idea that introspective skills are required to have phenomenal intuitions may take two different forms. First, it might be argued that people need to be trained to gain these introspective skills, and only after such training are they likely to come to hold the intuitions at issue. Second, one could be sceptical that these skills can be readily trained but hold that some people just naturally have them and that they are the ones who should be examined. More specifically, it might be claimed that some people are naturally more inward-thinking than others and that the intuitions of such people should be given priority over those who are not inward-thinking. I will discuss these two forms in order.

Starting with the requirement of introspective training, I cannot imagine exactly what such introspective training would consist in. More importantly, why would a person need such specialized training to recognize the phenomenality of supposed phenomenal experiences and to 
judge that those experiences pose an epistemic gap if indeed phenomenal consciousness is pretheoretically obvious? For the sake of argument, let's imagine that one can be trained to better recognize the features of phenomenal experiences. Introspective training might be directed toward at least two different ends. First, it might be designed to help laypeople recognize the peculiar, "phenomenal" features of experiences (qualitativeness, ineffability, atomicity and so on). Alternatively, such training might simply aim to teach people how to introspect on their phenomenal experiences more carefully or more skilfully.

Let's consider first the possibility that the trainee, after the training, starts recognizing, for example, that seeing colors, feeling pain, and so on have something in common because there is something that it is like to be in those states. In this case, we could not be sure that this "recognition" is due to the indoctrination the training involved, or due to the pure intuitions triggered with the help of the introspective training. This is because, it is really difficult to imagine, at least for me, that careful/skilful introspection somehow helps one to recognize the features of phenomenal states that are thought to distinguish them from other states. Let's consider the core "phenomenal" feature, namely qualitativeness. It is difficult to see how one could be trained to recognize the qualitative aspect involved in, say, seeing colors if this aspect is not already obvious to the trainee prior to training. In line with this, remember that Block states that "if you got to ask [what is this qualitative aspect], you ain't never gonna get to know" (Block, 1978, p. 281). This implies that if the qualitativeness of phenomenal experiences was not already obvious to you, you would never be able to recognize it by some explanations or training. Further, the notion of qualitativeness is itself unclear, such that it is unclear how the trainer could offer instruction that the trainee would recognize. Even when a hardworking trainee claims to finally recognize this aspect, it would seem quite plausible that the trainee and trainer could never be sure that they are talking about the same thing. And the same worries would seem to equally apply to other features of so-called phenomenal states such as intrinsicality, atomicity (e.g. yellowness seems to have no underlying parts) and ineffability (e.g. that yellowness cannot be described to a person who has 
not seen any yellow object). ${ }^{108}$ In brief, this type of training would be biased, at best, in the sense that the trainee is indoctrinated to think or claim that so-called phenomenal states have some special features, in which case we would have every right to doubt that the thought or claim of the trainee is due to her intuitions and that the trainer and the trainee mean the same thing by these special features.

However, perhaps one does not need such biased introspective training to have phenomenal intuitions. That is, perhaps it is possible to teach people how to carefully introspect on their (supposed) phenomenal experiences without setting a target for them to recognize or learn, and this might be enough to make people more likely to have phenomenal intuitions. So, this type of training would be unbiased. In this case, however, it would be ad hoc to assume that the trainee would recognize the peculiar features that phenomenal experiences are supposed to have. For one thing, as shown in $\$ 4.1$, laypeople tend to attribute the qualities we are acquainted with in perceptual cases and bodily sensations (such as colors and pains) to the objects perceived outside the mind (such as colored objects and the damaged part of the body). And there seems no reason to assume that, after learning how to carefully introspect on her experiences, the trainee would give up doing so and ascribe such qualities to the experience itself without specifically being taught such a theoretical view. Even if the naïve realist intuition that so-called phenomenal qualities belong to the objects perceived outside the mind were not prevalent, the objector would owe us an account of why such unbiased training should be expected to make a considerable difference.

So, the assumption that introspective training is needed for one to have phenomenal intuitions seems highly problematic. When the training is biased, we would have the right to be suspicious

\footnotetext{
${ }^{108}$ Although it is not very clear what philosophers mean by phenomenal states having intrinsic properties, following the rough definition of Marshall and Weatherson (2018, para. 1), what seems to be at issue is that phenomenal states are thought to have some properties that they have "purely in virtue of the way" they are rather than in virtue of the way they interact with the world. So, claiming that phenomenal states have intrinsic properties implies that phenomenal states have some properties that are not relational or representational. That is, phenomenal states have these properties not because of their relations (to other states etc.) or being a mental representation of the perceived world but purely because of the way they are. This in turn implies that even if you explain all the relational or representational properties of phenomenal states, the very way that a phenomenal state feels would remain unexplained (see Tye (2018)'s discussion of the intrinsicality of qualia for details).
} 
that the trainee was indoctrinated into judging that she has the right intuitions after training. And when it is unbiased, it is ad hoc to claim that such training would lead one to recognize phenomenality. Now, I will proceed to discuss the second form of the idea that introspective skills are required to have phenomenal intuitions.

One can accept that introspective training would not make a considerable difference or would nudge people towards thinking in a particular way and thus distort their judgements, and yet still believe that the people with more introspective skills are more appropriate to assess phenomenal theses. For example, Chalmers makes a distinction between inward-directed people who can "easily attend to their mental lives" and outward-directed people who "have trouble attending to the qualities of their conscious experience" and states that, perhaps, inwards-directed people are more likely to have phenomenal intuitions (Chalmers, 2020, p. 251). Fortunately, whether inwarddirected people are more likely to have phenomenal intuitions is an empirical question, and there is a study that provides us with an answer to this question. As noted in $§ 7.2 .2$, Study $2 \mathrm{~b}$ in Díaz (2021) looked at the correlation between measurements of how inward-thinking a person is and one's having metaphysical and explanatory gap intuitions. ${ }^{109} \mathrm{He}$ found no significant effect of inward-directedness on problem intuitions. This suggests that inward-directedness does not play a significant role for other types of phenomenal intuitions either. One could, of course, claim that Diaz's measurement of inward-directedness is problematic but there seems no reason to assume that the hypothesis that the inward-directed people are more capable of having phenomenal intuitions is true unless and until positive empirical evidence is provided for it.

In brief, the idea that when one carefully/skilfully introspects her so-called phenomenal experiences, whether by training or naturally, she could become more likely to have phenomenal intuitions seems groundless. Further, there is also the possibility that when one is exposed to introspective training or is more inward-thinking, she might come to have the opposite, antiphenomenal intuitions, as it were. For example, colors might seem to belong to the perceived object

${ }^{109}$ As noted in 7.2.2, the level of inward-thinking in Diaz's study was measured by the responses to some statements
such as "Being in touch with emotions is essential" and "I can feel close to someone, even in moments of silence". 180 
or the light when the trainee (or a person who is naturally more inward-thinking) introspects on episodes of seeing colors more carefully.

\section{Are New Concepts Required to Have Phenomenal Intuitions?}

We have seen that it is ad hoc to claim that one needs (biased or unbiased) introspective training to come to have phenomenal intuitions, or that inward-thinking people are more appropriate to consider in assessing whether philosophers' intuitions provide compelling evidence for phenomenal theses. That is, we have good reasons to believe that the first version of Requirements Objection fails. What about the idea that one needs to learn some relevant concepts to have phenomenal intuitions, which is the second version of Requirements Object?

The first issue for this version of the Requirements Objection is that it is unclear why we should think that people need explicit (rather than tacit) concept(s) to have phenomenal intuitions. If phenomenal consciousness is pretheoretically obvious, we would expect laypeople to have a tacit conception of phenomenality by which one who does not have any explicit concepts concerning phenomenal consciousness could still judge that a simple robot cannot feel pain and see colors, for example. In line with this, as we saw in Chapters 2 and 3, many (if not most) philosophers tend to think that laypeople share phenomenal intuitions without mentioning any requirement of learning new concepts.

Of course, without explicit concepts, one cannot form judgments that involve those concepts. For example, one cannot form the judgment that colors, and pains pose an insuperable problem for the brain sciences because they have phenomenal properties without having the concept of phenomenality. But why would one need to have explicit concepts (such as "phenomenality", "what-it-is-likeness", and so on) to make related judgements that do not involve such technical concepts, such as the judgement that it is possible that our physical and behavioural duplicates cannot feel pain? In the same vein, one does not need to gain any new concepts to classify phenomenal experiences as being similar and distinguish them from non-phenomenal experiences. As Chalmers holds, the specific "phenomenal" concepts such as feeling pain and seeing colors that untrained laypeople typically have would seem to be sufficient to have problem intuitions 
(Chalmers, 2018, p. 13; 2020). To put the same point with an analogy, one needs to learn the concept of TRIANGLE to form some judgements that involve this concept such as "triangles have three sides". However, one does not need to have the concept of TRIANGLE to see the commonality in triangles, classify different triangles as being similar, distinguish them from rectangles, or form judgements reflecting that triangles have different shapes than rectangles. So, there seems no reason to assume that possessing any new explicit concept would make one better in these tasks.

One might push the point and predict that learning some concepts about phenomenal consciousness (such as "what-it-is-like-ness", "raw feel", and so on) would make one more likely to have phenomenal intuitions because introspecting on phenomenal experiences in light of these concepts would help people make better judgments about their phenomenal experiences. This is, however, another way of claiming that one needs biased (conceptual) training to be likely to have phenomenal intuitions. And as noted, the worry is that during biased training people will be indoctrinated into a theoretical view about states like seeing colors and feeling pains. To repeat, biased training would render it unclear whether the judgements of the trainee that are suggestive of phenomenal theses hold because of her pure intuitions or because of a certain sort of authority bias that led the trainee to those particular judgments.

To summarize, if phenomenal consciousness is phenomenologically obvious, one should not need to learn any new concept about phenomenal consciousness to have the relevant intuitions. Further, learning such concepts might distort one's intuitive judgments about subjective experiences. Based on these considerations about the requirement of special introspective skills and new concepts, Assumption 1 seems safe to hold. However, establishing Assumption 1 alone is not sufficient to show that the general data provided by the studies discussed in Chapters 4-8 are relevant to assess whether philosophers' intuitions make their phenomenal theses compelling. We also need to show that Assumption 2 is plausible, which the next section aims to do. 


\subsubsection{Defence of Assumption 2}

Remember that Assumption 2 is that the level of reflectiveness is not a significant factor in having phenomenal intuitions. Against Assumption 2, a critic might point out the possibility that carefully reflecting on and thinking about the questions asked in the studies discussed above might make one more likely to respond in ways consistent with having phenomenal intuitions. Along these lines, Talbot (2012) charges that the studies in SM captured System-one judgements (quick, snapjudgements) rather than System-two judgements (slower, more thought-out judgements). Talbot claims that System-two judgements, rather than System-one judgements, are appropriate for investigating whether laypeople distinguish phenomenal states from other states. The same logic can be applied to the investigation of whether people think that there is an epistemic gap or not. And if reflective people - people who pay more attention to the questions in the surveys or give the questions more consideration - are better than non-reflective people in making judgements about phenomenal experiences, the critic contends that reflective people, by and large, will give responses that correspond with the phenomenal theses. And if this is the case, any empirical study investigating the prevalence of phenomenal intuitions should examine the responses of more reflective participants. In line with this, Chalmers, claims that at least some phenomenal intuitions are "present in a good majority of people, at least among reflective people in contemporary Western cultures" (Chalmers, 2020, p. 228).

Based on these considerations, one might deny Assumption 2 by claiming that since the studies discussed analysed all the responses rather than the responses of reflective people, the results of these studies are not relevant to assessing phenomenal theses. Let's call this the "Reflection Objection".

Note that the Reflection Objection relies on the hypothesis that the more carefully one thinks about a question concerning so-called phenomenal experiences, the more likely one is to respond in accordance with phenomenal theses. However, since there seems no easy way to measure how reflective a participant is while she is answering a particular question, the objector should at least assume that the participants who seem to pay more attention to the questions are more likely to 
give responses in favour of phenomenal theses. If this assumption fails, then the objection would be unsupported. One plausible and common way to test the assumption is to ask participants some questions that measure their tendency toward reflection in addition to the questions about phenomenal experiences, and to compare the responses of reflective and non-reflective people. If there is no significant difference between the responses of reflective and non-reflective participants, then we can safely assume that the assumption on which the Reflection Objection relies does not hold.

Two existing studies and seven new studies discussed in the thesis suggest that reflectiveness plays no significant role in participants' responses to the clear majority of philosophical questions. In reply to Talbot's criticism to SM, in their first three studies, Sytsma and Machery (2012) tested the prediction that reflective people would be more likely to deny that the simple robot (Jimmy) can see colors if they make a distinction between phenomenal and non-phenomenal states. To measure the participants' level of engaging reflectivity, they asked participants three questions of Shane Frederick's (Frederick, 2005) Cognitive Reflection Test (CRT), which, as noted before, is a standard test used for this purpose. None of these three studies indicated that the CRT performance of participants showed a significant correlation with philosophical responses. Remember also that Study 2b in Díaz (2021) found that neither level of reflection nor how inwardthinking a person is showed a significant correlation with one's having metaphysical or explanatory gap intuitions. Finally, as reported in Chapters 5, 6, and 8 when we discussed Ambiguity Studies 1-3, Dimension Studies 1-2 and Explanatory Studies 1-2, we found that there is no significant correlation between participants' responses to the philosophical questions and their CRT/CRT 2 scores for the great majority of questions. The correlations we found were mostly negligible and none of them applied to both CRT and CRT 2 test scores. In light of these findings, the assumption that reflective people are more likely to have phenomenal intuitions seems groundless.

The considerations presented in this section and the previous one suggest that there is no good reason at present to doubt Assumption 1 and 2. So, it seems safe to think that the general data 
provided by the studies discussed in the thesis are relevant to assessing whether philosophers' intuitions provide compelling evidence for phenomenal theses.

Showing that Assumption 1 and Assumption 2 hold is not enough to establish Premise 3, however. We also need to show that if Premise 2 and these assumptions hold, then any way of appealing to phenomenal intuitions fails to provide compelling evidence for phenomenal theses. That is, the defence of Premise 3 calls for an explanation of why appealing to phenomenal intuitions would fail if phenomenal intuitions are not prevalent in general and of why the prevalence of phenomenal intuitions in general samples is relevant to assessing phenomenal theses. The next two sections aim to do this.

\subsection{Against the Collectivist Way of Appealing to Phenomenal Intuitions}

As discussed in \$2.3.1, philosophers may appeal to phenomenal intuitions in two different ways to support phenomenal theses: collectivist or individualist. So, to establish Premise 3, we need to show that both the collectivist and the individualist ways of appealing to phenomenal intuitions fail to provide compelling evidence for phenomenal theses. In this section, I will argue that the collectivist way fails while the next section will argue that the individualist way also fails.

Remember that in the collectivist way of appealing to phenomenal intuitions, philosophers can simply appeal to the wisdom of the crowd and claim that phenomenal intuitions being widespread speaks in favour of phenomenal theses. This might seem to be an unattractive approach, taking it to suffer from an ad populum fallacy, especially considering that science has shown many times that even the most prevalent intuitions are sometimes mistaken (such as the intuition that speed of time is fixed). Yet philosophers may plausibly claim that although the judgement of the majority is fallible, we should still consider such judgements to be plausible by default. One sensible reason for this might be that if the majority of people observes that $\mathrm{P}$, we can safely assume that $\mathrm{P}$ unless countervailing evidence is provided. For example, we should accept that there is a cat at the corner of a street if, say, 8 out 10 people see that there is a cat at the corner of the street unless it is proven that the majority of observers are mistaken because of some reasons that lead to mass illusion or hallucination. In the same vein, assuming that phenomenal intuitions are due to introspection of 
phenomenal experiences, which can be treated as a sort of (inner) observation, one can reasonably claim that we should accept what almost all people recognize or "observe" regarding subjective experiences.

Alternatively, the philosopher who follows the collectivist way can claim that phenomenal intuitions being prevalent indicates that these intuitions are a natural result of the existence of phenomenal consciousness, which would then explain the intuitions being strong and widespread. In such a case, phenomenal theses would at least be intuitively plausible although it could nonetheless be that these intuitions are due to some systematic illusions.

However, we have seen that we have good evidence suggesting that most people seem to lack experiential property, common aspect, conceivability, and explanatory gap intuitions. So, if one accepts Premise 2, one cannot defend phenomenal theses by relying on the prevalence of phenomenal intuitions. This is because appealing to the wisdom of the crowd would not work for phenomenal theses but against them! And if phenomenal intuitions are not prevalent, the assumption that phenomenal intuitions are a natural result of phenomenal consciousness would not seem to be supported. So, the non-prevalence of phenomenal intuitions straightforwardly undermines the collectivist way of appealing to phenomenal intuitions.

\subsection{Against the individualist way of appealing to phenomenal intuitions}

What about the individualist way of appealing to intuitions? A philosopher can plausibly say that it is her strong phenomenal intuitions, rather than the prevalence of those intuitions, that justifies her belief in phenomenal theses. ${ }^{110}$ So, why would the non-prevalence of those intuitions make phenomenal theses less convincing?

\footnotetext{
${ }^{110}$ As a matter of fact, even if the philosopher relies purely on her intuitions, it is still controversial that the philosopher is justified. A reliabilist, who basically holds that a justified belief should be formed by a reliable process, might object, for example, that intuition-based beliefs, unlike the beliefs gained based on perceptions, are not rationally warranted beliefs. A less radical reliabilist objection might be that not all intuitions, but just the intuitions that are not shared by the majority of people, are not reliable enough to make a belief justified.
} 
In reply, it is important to see that Premise 3 is not about the epistemic justification of phenomenal theses. That is, it does not claim that one is not justified in believing in phenomenal realism or the epistemic gap thesis. The premise instead concerns a claim about whether intuitions of the proponents of phenomenal theses should be compelling or not for a neutral, unconvinced person. It claims that if phenomenal intuitions are not prevalent, the intuitions of the minority would not qualify as compelling evidence. There are three main reasons to think that the intuitions of the minority do not provide compelling evidence in favour of phenomenal theses for those who are not convinced about those theses, which will be presented as three considerations below.

\subsubsection{First Consideration}

First, there is a dilemma for the philosopher who follows the individualist path (let's call her an "individualist" for convenience). The individualist may contend, or not, that phenomenal consciousness plays a role in producing phenomenal intuitions. Let's first assess the second option, considering an individualist who does not assume that phenomenal consciousness plays a role in producing phenomenal intuitions. In this case, the individualist would have trouble explaining why her intuitions are suggestive of the existence of phenomenal consciousness if there is no link between phenomenally conscious processes (or our acquaintance with phenomenal qualities) and phenomenal intuitions. After all, she may then have phenomenal intuitions regardless of whether

phenomenal consciousness exists or not. So, for the individualist to plausibly claim that her intuitions are suggestive of phenomenal realism and/or the epistemic gap, she would need to assume that phenomenal consciousness plays a role in producing phenomenal intuitions. This role might be a causal role with phenomenally consciousness states and/or our acquaintance with phenomenal qualities causing phenomenal intuitions. And perhaps one can imagine other types of links between phenomenal consciousness and phenomenal intuitions; but in any case, however, this link is specified, phenomenal consciousness would play a role in producing phenomenal intuitions.

If there is such a link by which phenomenal consciousness plays a role in producing phenomenal intuitions, however, and if the majority of people are not philosophical zombies (if they are 
phenomenally conscious creatures), then the individualist needs to explain why the majority of people seem to lack phenomenal intuitions.

One can accept that phenomenal intuitions are not widespread and yet can still claim that it does not follow that there is no link between phenomenal consciousness and phenomenal intuitions. On this idea, when a phenomenal intuition is present, this is in virtue of phenomenal consciousness regardless of whether or not phenomenal intuitions are widespread. Along these lines, Chalmers asserts that there are near-universal factors-e.g. mental states and introspective mechanisms that most humans share - that "play a central underlying role in explaining problem intuitions where they are present," although these consciousness-related factors might be overwhelmed by more variable factors, such as "cultural, linguistic, and theoretical background, and other factors that vary with historical period and individual psychology") (Chalmers, 2018, p. 15). So, it can be claimed that if one has phenomenal intuitions, near-universal, phenomenal-consciousness related factors played a role in creating them but if one does not have (or does not seem to have) phenomenal intuitions, then this is because of variable factors.

I have already discussed the most plausible variable factors that Chalmers has in his mind in relation to the objections above: one might be more inward-thinking or reflective than others, or one might have a belief that conflicts with one's phenomenal intuitions. To repeat very briefly, inward-thinkingness and reflectiveness do not appear to be significant factors with regard to having phenomenal intuitions, and there does not seem to be a good reason to assume that having conflicting beliefs blocks phenomenal intuitions rather than promoting them. So, it is ad hoc to attempt to explain why the majority of people seem to lack phenomenal intuitions by appealing to variable factors. And it seems difficult for the individualist to explain why phenomenal consciousness does not produce phenomenal intuitions (or reports that reflect them) if there is an organic link between phenomenal processes or qualities and phenomenal intuitions, considering that none of the most promising candidates is very helpful for such an explanation.

What is worse for the individualist is that the scarcity of phenomenal intuitions gives us good reason to suspect that the phenomenal intuitions of the individualist (or of the minority, more 
generally) are due to reasons that have nothing to do with phenomenal consciousness. That is, it gives us reason to suspect that there might be variable factors that are operating in the reverse way to what Chalmers suggests: Having phenomenal intuitions might be due to variable factors that have nothing to do with phenomenal consciousness.

But how exactly? Remember that when I discussed the collectivist way of appealing to phenomenal intuitions, I stated that phenomenal intuitions being widespread provides defeasible support for the possibility of a link between phenomenal consciousness and phenomenal intuitions. But if the prevalence of phenomenal intuitions is suggestive of the existence of such a link, then the scarcity of them is suggestive of the inexistence of that link. If one objects that the scarcity of phenomenal intuitions does not suggest this, then equally one would not be in a position to plausibly claim that the prevalence of phenomenal intuitions would suggest that there is such link.

Perhaps the individualist thinks that the prevalence or scarcity of phenomenal intuitions does not suggest anything about whether there is a link between phenomenal consciousness and phenomenal intuitions, however. In this case, though, the individualist would be in trouble if she insists that her phenomenal intuitions are suggestive of the existence of such a link. After all, if the majority of people having phenomenal intuitions does not suggest anything about whether phenomenal consciousness plays a role in producing phenomenal intuitions, why should we believe that the individualist's intuitions suggest anything about the same issue? So, there seems to be no plausible way for the individualist to deny that phenomenal intuitions being rare provides defeasible support for the possibility that the phenomenal intuitions of the individualist are due to variable factors rather than near-universal factors related to phenomenal consciousness. This straightforwardly undercuts the individualist way of appealing to phenomenal intuitions because we can now assume that the phenomenal intuitions of the individualist would indicate nothing about the existence of phenomenal consciousness, no matter how strong they are.

To summarize the first consideration in slightly different terms, the only plausible way for the individualist to claim that her phenomenal intuitions suggest that phenomenal consciousness exists is to assume that the occurrence of phenomenal intuitions is linked to phenomenal consciousness. 
But if this is the case, then we would expect the majority of people to have phenomenal intuitions. However, if Premise 2 is accurate, they do not! This makes the claim that phenomenal consciousness plays a role in producing phenomenal intuitions less likely in just the same way as the claimed prevalence of phenomenal intuitions would make the claim more likely. So, we're warranted in assuming that the phenomenal intuitions of the individualist are due to factors that have nothing to do with phenomenal consciousness. And if this is the case, then the phenomenal intuitions of the individualist do not suggest in favour of the existence of phenomenal consciousness and thus do not provide compelling evidence for phenomenal theses.

\subsubsection{Second Consideration}

The second reason to think that the individualist way of appealing to phenomenal intuitions fails is that if the majority lack phenomenal intuitions and if the minority and majority are equally competent with regard to the questions in the empirical studies, then there is good reason for a neutral person to discredit the individual intuitive evidence of proponents of phenomenal theses. Such a neutral person would be completely rational if she held that the individual intuitive evidence of the minority is not enough to push her to believe in phenomenal theses. A neutral person would perhaps be irrational to believe that phenomenal theses are wrong because most people do not confirm the evidence on which these theses rely, but it would be perfectly rational for such a person to reach the verdict that there is no compelling (intuitive) evidence to believe in phenomenal theses because only the minority share phenomenal intuitions.

Consider the following analogy: If two witnesses report that Albert murdered someone and eight witnesses report that Albert was not at the crime scene, it would be rational for a juror to arrive at the verdict that Albert is not guilty. The verdict does not mean, of course, that Albert is innocent. It means that there is not enough evidence to conclude that Albert is guilty. In other words, it would be irrational for the juror to think that the reports of the two witnesses who claim to have seen Albert committing the crime provide compelling evidence against Albert in light of the conflicting reports of the other eight witnesses. Similarly, it would be irrational for a neutral person to judge that the intuitions of the minority provide compelling evidence for phenomenal theses. 
Note that this reasoning assumes that the minority whose responses accord with phenomenal theses and the majority whose responses do not are epistemic peers regarding their judgements about these topics. One could, of course, claim that participants in the minority and the majority are not epistemic peers. For this to be compelling, however, the objector should offer a reason for thinking that there are some factors that would make a considerable difference between the quality of the responses of the participants. For example, one participant might be more susceptible to blocking factors than the other. As noted, before, this would not help the objector, however, because there is no reason to think that blocking factors affect those who seem not to have phenomenal intuitions more than those who seem to have. Alternatively, in line with the Expertise Objection, one could claim that philosophers are more protected than others from such factors. We have already discussed this objection, though, and noted that a number of experiments undermine the idea that philosophers are in a safer position when it comes to confounding effects and biases.

\subsubsection{Third Consideration}

Finally, the third reason to think that the individualist way of appealing to phenomenal intuitions fails is that even the proponent of phenomenal theses should admit that her individual intuitive evidence is far from compelling. This is because (1) epistemic considerations on peer disagreement suggest that a person should be less convinced about her belief that $\mathrm{P}$ when she learns that an epistemic peer rejects P; and, (2) she should be even more doubtful about her belief that P if the position of her opponent is much more common. To be more precise, let's imagine that Edgar believes that the physical sciences cannot ever fully explain feeling pain (P) because of his intuitions about feeling pain while Edgar's epistemic peer, Heathcliff, rejects $\mathrm{P}$ again by appealing to his intuitions about feeling pain. (1) claims that the epistemic considerations on the disagreement between Edgar and Heathcliff suggest that Edgar should be more doubtful or less convinced about his belief that $\mathrm{P}$ after learning that an epistemic peer (Heathcliff) rejects $\mathrm{P}$. Imagine further that $\mathrm{P}$ is rejected by the majority of people. (2) claims that in this case, Edgar should be more doubtful about his belief than Heathcliff. 
In defence of (1), I contend that no main epistemic position on peer disagreement would suggest that Edgar's doxastic attitude to P should not change at all after learning that Heathcliff rejects P. The Equal Weight View on peer disagreement (Bogardus, 2009; Christensen, 2007; Elga, 2007) claims that "learning that a peer disagrees with you about $\mathrm{P}$ gives you a reason to believe that you are mistaken about P" (Frances \& Matheson, 2019, 5.1 section, para. 2) and that epistemic peer should give equal credence to the opponent view and be less convinced about $\mathrm{P}$ after discovering disagreement. So, Edgar should be less sure about P after learning that Heathcliff rejects his view.

By contrast, the Justificationist View championed by Jennifer Lackey claims that in some "Extreme Disagreement" cases it would be rational for one to stick to her beliefs after learning that a peer disagrees with her. In these cases, she might be highly justified in believing $\mathrm{P}$ while the other party of the disagreement is not justified at all. A paradigm example is the disagreement between one who believes that $2+2=4$ and another person who believes that $2+2=5$ (Lackey, 2010, p. 283). Note that in this case the Equal Weight View would give the irrational verdict that one should be less convinced about $2+2=4$. So, perhaps the disagreement between Edgar and Heathcliff is this type of disagreement in which Edgar is highly justified in believing $\mathrm{P}$ while Heathcliff has little or no justification. And if this is the case, it would still be rational for Edgar to believe that $\mathrm{P}$ equally strongly even after learning that Heathcliff disagrees. However, it is not difficult to see that there is no reason to think that Heathcliff's judgement should be demoted because both parties appeal to the same type of intuitive data about feeling pain in epistemically equal conditions. That is, unlike the extreme disagreement cases, Edgar and Heathcliff are equally justified in their opposite intuitions.

A similar view, the Total Evidence View proposed by Thomas Kelly, claims that the Equal Weight View ignores the qualities of the (first-order) evidence of both parties by holding that disagreement should equally make the parties less sure about $\mathrm{P}$ and not-P (that the confidence levels of parties should be reduced equally). According to Total Evidence View, one party might appropriately appeal to the shared evidence while the other party misjudges the same evidence, or one party might have reliable evidence whereas the other has no such evidence. Kelly's example is that my belief that the Holocaust occurred is based on reliable evidence while my peer who denies the 
Holocaust is ignorant of this evidence (Kelly, 2013). Again, if the disagreement between Edgar and Heathcliff is similar to this type of case in which there is a big difference between the quality of the evidence, then it would be irrational for Edgar to be less sure about P. However, as noted, Edgar and Heathcliff appeal to the same type of intuitive evidence and there is no reason to think that one is in a better position than the other to evaluate their pieces of evidence.

The last and the most radical option that might support the claim that Edgar should not change his doxastic attitude towards P is the Steadfast View (Huemer, 2011; Van Inwagen, 1996). This view claims that at least in some cases peer disagreement does not require the peers to be less sure about $\mathrm{P}$. The rationale behind this view is that if I have access to a special body of evidence that my disagreeing peer lacks, or if my evidence is private and thus cannot be shared by my peer, then it would be reasonable for me to stick to my belief that $\mathrm{P}$. The main examples of private evidence are experiences and intuitions: If my peer cannot share my experiential or intuitive evidence then my peer lacks remarkable evidence, which suggests that my peer's rejection of $\mathrm{P}$ carry little or no weight. A plausible answer to the view is that if both parties have their own private evidence although they lack the private evidence of their peers, then they are epistemically equally advantaged in one sense and disadvantaged in another sense (Feldman, 2006). So, it is reasonable for the peers to be doubtful about their judgements about $\mathrm{P}$ after discovering that they disagree. Note that both Edgar and Heathcliff seem to base their opinions on the same type of private, intuitive evidence: it seems to one that the physical sciences can in principle fully explain feeling pain and to the other that the physical sciences cannot. So, there is good reason for them to be less sure about $\mathrm{P}$ or not-P unless it is proven that one of them is making a scandalous mistake in their judgements.

In brief, the case of Edgar and Heathcliff is not a case in which one's judgement should be given privilege over the other's judgement and thus one should not change his doxastic attitude to P. This is because both Edgar and Heathcliff are equally justified and appropriately rely on their private, intuitive evidence in their judgements. So, the main epistemic positions on peer disagreement suggest that the rational doxastic change towards P for both Edgar and Heathcliff is to reduce their confidence levels about their beliefs. Of course, one can think along the lines of 
another position on peer disagreement, but such an alternative view would need to find a way to explain why Edgar should put more weight on his belief despite the fact that Edgar and Heathcliff are epistemic peers, and that there seems no difference between them in their level of justification, the type of evidence they base their opinions on, or the quality of their evidence.

One can object that not only Edgar but also Heathcliff should be less sure about his belief after discovering they disagree. So, if Edgar's reduced confidence level makes his belief less convincing, the same applies to Heathcliff as well. However, it is crucial to see that if Heathcliff becomes less sure about not-P, this will not affect much the credibility of the opponent views (phenomenal anti-realism and epistemic gap denial). This is because the main motivation for these views comes from ontological and explanatory simplicity rather than intuitions. On the contrary, if Edgar became less sure about $\mathrm{P}$, the main motivation of phenomenal realism and thus the epistemic gap thesis would be compromised.

Further, as (2) points out - that is, that the individualist should be even more doubtful about her belief if the position of her opponent is much more common-there is a big difference between Edgar's and Heathcliff's position: Heathcliff's position is much more common. So, if equal weight should be given to everyone, it would be rational to think that Heathcliff's position deserves more credence. This is because if an individual intuition has evidential value, why should not we believe that more of them have more value? So, if Edgar thinks that his intuition provides compelling evidence for $\mathrm{P}$, he would be committed to thinking that there is more compelling evidence for notP. Note that in this reasoning the notion of compelling evidence is so liberal that the difference between the notions of compelling evidence and evidence evaporates. To see this better, imagine that there are ten possible positions on a controversial topic and each position is supported by ten different intuitions. Imagine further that $10 \%$ of people share each of these competing intuitions. If we use the notion of compelling evidence too liberally, as in the above reasoning, we would be committed to claiming that each of the ten positions has their own compelling evidence, which is clearly implausible. It would be more rational to think that although it can be said that each position has their own evidence in a loose sense of "evidence", no position has compelling evidence because the intuitive pieces of evidence of each group cancel each other out. When it comes to the 
case of Edgar and Heathcliff, there might be two rational doxastic attitudes towards $\mathrm{P}$ for Edgar after learning that the majority of people do not share his intuition. He should either think that the evidence for Heathcliff's position, which is supported by the majority, overweighs his evidence, or that his and Heathcliff's intuitive evidence cancel each other out. Either way, Edgar should admit that his intuitive evidence, if it is evidence at all, is weak and far from being compelling.

These three considerations give us good reason to think that the individualist's phenomenal intuitions do not provide compelling evidence for phenomenal theses if these intuitions are shared only by the minority of people. That is, the individualist way of appealing to phenomenal intuitions fails to give us a compelling reason to believe phenomenal theses. We have also seen that the collectivist way of appealing to phenomenal intuitions is straightforwardly undermined by the scarcity of these intuitions. So, both ways of appealing to phenomenal intuitions fail to provide compelling evidence for phenomenal theses, supporting the plausibility of Premise 3.

\subsection{Concluding Remarks}

As noted, Chapters 2-3 provided textual evidence to support Premise 1 of the General Argument, which I believe is the least controversial premise. So, showing that Premise 2 and 3 are plausible and that the argument is valid would be enough to show that the conclusion of the argument is also plausible, which was the aim of the current chapter.

We have seen first that the assumption behind Premise 2 is plausible. That is, the results of the empirical studies discussed in previous chapters provide us with a reliable, approximate measure of the prevalence of phenomenal intuitions. We have also seen that two assumptions behind Premise 3 are plausible. That is, there is no reason to doubt that the general data provided by the studies that were discussed in Chapters 4-8 and supported Premise 2 qualify as relevant data for assessing whether philosophers' intuitions make their phenomenal theses compelling. To put the same point in reverse, there is no reason to think that what matters is the prevalence of phenomenal intuitions among those who have some special introspective skills or concepts, or who are more reflective, rather than the general prevalence of phenomenal intuitions, for purposes of assessing how compelling phenomenal theses are. Finally, we saw that both the collectivist and individualist 
ways of appealing to phenomenal intuitions fail to make the phenomenal theses compelling, if Premise 2 is true.

What about the conclusion? The conclusion of the General argument holds that the primary (intuition-driven) way of defending phenomenal theses fails. It is not difficult to see that the conclusion is just the logical consequence of Premise 1 (that phenomenal theses are primarily defended by appealing to phenomenal intuitions) and Premise 3 (that appeals to phenomenal intuitions that are not prevalent do not provide compelling evidence for phenomenal theses). After all, if $\mathrm{E}$ is the main type of evidence to defend a thesis $\mathrm{T}$, and if $\mathrm{E}$ is not compelling, then the main way of defending $\mathrm{T}$ fails. That is, the General Argument is valid. And if the argument is valid, considering that the premises are plausible, then the conclusion is also plausible.

We are now in a position to conclude that the main (intuition-driven) way of defending phenomenal theses fails. This has some direct implications for the debate between phenomenal realism and anti-realism and thus for the mind-body problem. However, in the next chapter, I will focus only on the implications of the empirical evidence suggesting that phenomenal intuitions are not prevalent for the meta-problem of consciousness. 


\section{CONCLUSION}

Philosophers commonly assume that phenomenal intuitions are natural and widely shared. As we have seen across this thesis, however, there is ample evidence indicating against this assumption. Not only does it not appear to be the case that phenomenal intuitions are widely shared, but quite the reverse: the evidence suggests that such intuitions are scarce outside of philosophy. In this concluding chapter, I will first very briefly summarize this evidence and the General Argument that I have given. I then draw out some further implications of these findings, focusing on the socalled "meta-problem of consciousness". Chalmers (2018) introduces the meta-problem as the problem of explaining our intuitions about phenomenal consciousness. I argue that the evidence presented above deflates this problem. Given the scarcity of phenomenal intuitions, I charge that the real problem we need to come to grips with doesn't concern our intuitions about phenomenal consciousness, but our general lack of them. And this reverse meta-problem poses a serious challenge for phenomenal realists: Given the paucity of intuitive support for the existence of phenomenal consciousness, why should we believe that it exists in the first place?

\subsection{A Brief Summary of the General Argument}

This thesis is motivated by the idea that whether phenomenal intuitions are prevalent or not has important implications for prominent philosophical claims about phenomenal consciousness. If (1) phenomenal theses are typically defended by appealing to phenomenal intuitions, and if (2) phenomenal intuitions are not prevalent, then it is reasonable to hold that (3) appealing to phenomenal intuitions does not provide compelling evidence for phenomenal theses. Taking (1), (2) and (3) as its premises, the General Argument concludes that the main way of defending phenomenal theses fails.

I have supported the premises of the General Argument step by step. In support of the first premise, Chapters 2 and 3 detailed the central role of intuitions in standard philosophical defences of phenomenal realism and the epistemic gap thesis. To summarize, it would not be an exaggeration

to say that if we dropped the works that (directly or indirectly, implicitly or explicitly) appeal to 
phenomenal intuitions in arguing for phenomenal theses from the literature, few works, if any, would remain.

The second premise is clearly an empirical premise. It is the most controversial premise and the backbone of the argument. So, establishing this premise formed the core of the thesis. Chapters 46 discussed both existing and new studies investigating the prevalence of existence intuitions (experiential property and common aspect intuitions) while Chapters 7-8 did the same for two major types of epistemic gap intuitions (conceivability and explanatory gap intuitions). I will summarize the empirical evidence discussed in these chapters in relation to the meta-problem in the next section. I argue that these empirical studies make a strong case for Premise 2, indicating that neither existence nor epistemic gap intuitions are prevalent. That is, phenomenal intuitions that appear to be common among philosophers are not shared by the majority of laypeople.

Finally, Chapter 9 first defended Premise 2 and Premise 3 against some general objections, then aimed to establish Premise 3 by arguing that both collectivist and individualist ways of appealing to intuitions fail to provide us with compelling evidence for phenomenal theses, accepting that phenomenal intuitions are not prevalent. The chapter concluded that since the General Argument is valid, and the premises are individually plausible, the conclusion of the argument is also plausible.

The conclusion of the argument - that the main way of defending phenomenal theses fails - has some major implications for the debate between phenomenal realism and anti-realism and thus for the mind-body problem. If the main, intuition-driven way of defending phenomenal realism fails, this means that in the absence of compelling, non-intuition-based evidence in favour of phenomenal realism, the existence of phenomenal consciousness is far from being a datum that should be assumed. On the contrary, it can be plausibly argued that phenomenal anti-realism should be assumed true because of its ontological parsimony and explanatory simplicity. And if we should assume that phenomenal consciousness does not exist, then we should also assume that there is no epistemic gap and, thus, no metaphysical gap between the phenomenal and the physical. This undermines the foundations of the classic anti-physicalist reasoning and, as such, supports 
physicalism. In the remainder of this chapter, I turn to a further, related set of implications of this thesis, focusing on what the scarcity of phenomenal intuitions means for the so-called metaproblem of consciousness.

\subsection{Implications for the Meta-Problem of Consciousness}

Chalmers (2018) introduces a new research programme under the name of the "meta-problem of consciousness". ${ }^{111}$ This paper has already received much attention, and not only from philosophers of mind but also from scientists working on phenomenal consciousness. As will be explained, the problem relies on some empirical hypotheses about the prevalence of phenomenal intuitions. So, the empirical data discussed in this thesis are directly relevant to this supposed problem. In this section, I will discuss the implications of the scarcity of phenomenal intuitions for the metaproblem.

Chalmers, to a first approximation, defines the meta-problem of consciousness as the problem of explaining why we think (or have intuitions that reflect that) phenomenal consciousness poses a hard problem (p. 6). I will call this initial definition the "presumptuous definition" since it presumes that "we" have intuitions that there is a hard problem (problem intuitions in Chalmers's terminology). However, as a second approximation, he gives us a more modest definition: the meta-problem is the problem of explaining problem intuitions or reports in topic-neutral terms"roughly, terms that do not mention consciousness (or cognate notions such as qualia, awareness, subjectivity, and so on" (p. 16).

Chalmers defines problem intuitions also in terms of dispositions to make problem reports and judgements, such as verbally reporting or judging that feeling pain cannot be fully explained by

\footnotetext{
${ }^{111}$ Chalmers (2018) states that "The meta-problem opens up a large and exciting empirical and philosophical research programme" (p. 10) . However, how "new" this research programme is unclear. As Chalmers points out, "the metaproblem has a long history" (p. 8) because the research topic of the meta-problem, namely our intuitions about phenomenal consciousness, has been discussed for a long time. Further, phenomenal intuitions have been focused on in many different studies in experimental philosophy of consciousness, including the studies I've discussed across this thesis.
} 
neuroscience (p. 11). He lists four central types of problem intuitions: explanatory, metaphysical, knowledge, and modal intuitions (p. 12), and makes the empirical claim that problem intuitions are widely shared (p. 15). However, he notes that "Human intuitions and reports about the mind are plausibly produced by a combination of near-universal factors (e.g. mental states and introspective mechanisms that most humans share) and more variable factors (e.g. cultural, linguistic, and theoretical background, and other factors that vary with historical period and individual psychology)", and "variable factors will yield a great deal of variation in reports and intuitions, and may sometimes overwhelm the contribution of more universal factors" (p. 15).

Chalmers seems to imply that if his empirical hypothesis about the prevalence of problem intuitions is incorrect, this can be explained by variable factors that prevent problem intuitions from occurring or being reported. However, in any case, his working hypothesis is that there are near-universal factors related to phenomenal consciousness that play a central, underlying role in producing problem intuitions and reports (p. 15).

In what follows, I will pursue the implications of the empirical evidence discussed in Chapters 48 for the meta-problem. Let's first discuss what the empirical studies discussed in the thesis imply for the prevalence of problem intuitions for purposes of assessing Chalmers's empirical and working hypotheses, providing a brief summary of the positive evidence that I called on to support Premise 2 of the General Argument.

\subsubsection{Implications of the Scarcity of Existence Intuitions on Meta-Problem}

Remember that there are two main types of existence intuitions - that is, intuitions that are suggestive of phenomenal realism - experiential property intuitions and common aspect intuitions. Experiential property intuitions reflect that sensory qualities such as redness are properties of our experiences. Common aspect intuitions reflect that there is something that it is like to be in some mental states (seeing colors, feeling pain and so on) but not others (believing, knowing, making inferences and so on), and relatedly that some entities (such as humans, dogs, bats and other complex animals) but not others (artefacts such as calculators, thermostats, simple robots and so on) can have these states. As we have seen, and as I will briefly summarize below, a range of 
studies indicate that neither experiential property intuitions nor common aspect intuitions are prevalent in the general population.

Chalmers's list of central problem intuitions does not include existence intuitions. However, he mentions "distribution intuitions" and "quality intuitions" as being nearby. Distribution intuitions concern which systems do and do not have phenomenal consciousness, such as the intuition that robots have no phenomenal life. Quality intuitions concern the "special" qualities such as "colors and the like" (pp. 12-13) that phenomenal experiences supposedly have. In this subsection, I will first show that the studies that suggest that existence intuitions are not prevalent also suggest that distribution and quality intuitions are not prevalent. I will then argue that although these two types of intuitions are not considered to be central intuitions by Chalmers, the scarcity of these intuitions casts significant doubt on the prevalence of what he considers to be central problem intuitions.

Let's start with common aspect intuitions. Recall that in the first study in Sytsma and Machery (2010; SM), lay participants, unlike philosophers, by and large, ascribed seeing colors to a simple robot while denying that it felt pain. That is, the study suggests that laypeople treat two paradigmatic types of phenomenal states quite differently. Sytsma and Machery conclude from this that laypeople do not tend to employ something like the philosophical concept of phenomenal consciousness. Remember also that this conclusion is supported not only by a range of follow-up studies, including the new studies I presented in Chapter 5, but also by recent work on the dimensions of mind perception discussed in Chapter 6. Across these studies, researchers found that prototypical examples of supposed phenomenal states fall across different dimensions. In line with SM's findings, these results again suggest that laypeople do not treat paradigmatic types of phenomenal states similarly, and thereby further indicating that common aspect intuitions are not prevalent. That laypeople by and large lack common aspect intuitions - that they don't treat there as being something important in common between different prototypical examples of supposed phenomenal states-indicates that they do not employ a concept suitably similar to the philosophical concept of phenomenal consciousness. And this, in turn, indicates that distribution intuitions are not prevalent, since if people do not have a conception of phenomenality, then they 
cannot have intuitions about the distribution of phenomenal consciousness across different systems.

It is not clear exactly how "quality intuitions" are meant to be understood, but I take the intent to be to capture what we might call "qualia intuitions"-intuitions that "colors and the like" (Chalmers, 2018, p. 13) are phenomenal qualities or that they have special features constitutive of phenomenal qualities (e.g., mind-dependency, qualitativeness, intrinsicality, atomicity). Interpreted in this way, it is not difficult to see that the same two sets of studies mentioned above suggest that laypeople do not recognize the special, "phenomenal" commonality shared by perceptual states and bodily sensations. That is, they do not treat "colors and the like" as phenomenal qualities, which is equivalent to saying that they lack quality intuitions.

Further, the studies indicating the scarcity of experiential property intuitions provide us with additional evidence that quality intuitions are not prevalent. As discussed in §4.1, Sytsma (2010), Reuter and Sytsma (2018) and Kim et al. (2016) present evidence indicating that laypeople mostly locate colors and pains in the objects outside the mind and, relatedly, that they believe that there can be unfelt pains. This indicates that laypeople do not tend to share experiential property intuitions. This in turn suggests that they do not treat colors and pains as being special in the sense of being experiential and, thus, that quality intuitions are not prevalent.

In light of the evidence noted above, it seems reasonable to say that laypeople by and large do not share distribution and quality intuitions. However, what is more important for present purposes is that the scarcity of existence intuitions also casts significant doubt on the prevalence of the "central" problem intuitions that Chalmers focuses on. This is simply because the paucity of existence intuitions suggests that laypeople do not have something suitably similar to the philosophical concept of phenomenal consciousness, and if they do not have such a concept, then they would not seem to be able to intuit that phenomenal consciousness poses a hard problem. After all, one could not problematize something that one did not even conceptualize in the first place. To be more specific, if people do not have such a conception of phenomenal consciousness, 
it would be quite strange to expect them to have intuitions that qualia are metaphysical or that there is an epistemic gap between the phenomenal and the physical.

Unsurprisingly, Chalmers disagrees. In introducing the meta-problem of consciousness, he dismisses the studies presented in KP and SM as mainly investigating distribution intuitions, writing:

Where consciousness is concerned, the largest body of research concerns the distribution of consciousness (e.g. Gray, Gray and Wegner, 2007; Knobe and Prinz, 2008; Sytsma and Machery, 2010): for example, do people think that machines or corporations can feel pain? Some attempts have been made to connect this research to the hard problem of consciousness, but for the most part the intuitions in question have not been the core problem intuitions (Chalmers, 2018, p. 14).

However, as noted in Sytsma and Ozdemir (2019), a major concern of both KP and SM is whether laypeople have a concept that suitably resembles the philosophical concept of phenomenal consciousness and they simply use machines and corporations as comparison cases for investigating this (p. 243). As such, Knobe and Prinz conclude that laypeople have a conception of phenomenality while Sytsma and Machery conclude that they do not. And as noted above, whether there is a lay conception of phenomenality or not is directly relevant to the question of whether laypeople could have intuitions that phenomenal consciousness poses a hard problem. As such, SM's conclusion can be read as the claim that it cannot be the case that problem intuitions are widespread given that the philosophical concept of phenomenal consciousness is not widespread in the first place.

Being aware of the connection between SM's conclusion and problem intuitions, Chalmers claims that SM at most shows that laypeople do not have a unified concept of phenomenal consciousness (Chalmers, 2018, p. 14). He remains neutral concerning whether people have such a unified concept, but predicts that many or most people "have concepts of specific phenomenal states such as feeling pain or experiencing color" (p. 14). Further, he points out that the most common problem 
intuitions involve these specific phenomenal concepts rather than a unified concept of phenomenal consciousness.

In reply, as we noted in Sytsma and Ozdemir (2019, p. 244), SM's conclusion does not need to be read as the claim that laypeople do not have a unified concept of phenomenal consciousness. Rather, SM concludes that people do not recognize the phenomenality of two paradigmatical "phenomenal states"- seeing colors and feeling pain. That is, laypeople by and large do not even have "concepts of specific phenomenal states" simply because they do not treat the states Chalmers takes to be phenomenal as being phenomenal states! And if this is the case, then we should not expect laypeople to report any problem intuitions because they lack the specific phenomenal concepts needed for problem intuitions. To follow Chalmers's example, since laypeople generally do not treat seeing colors as phenomenal experiences, we should expect them to find color inversion cases unrealistic, and in fact this is exactly what Sytsma (2010) found.

Chalmers (2018, p. 13) also replies to SM's conclusion along the lines of the Ambiguity Objection, as follows:

In fact this result [the findings of SM] is predicted by Chalmers (1996, p. 18), which observes that ordinary mental terms like this have both a functional reading and a phenomenal reading, with sensational terms such as 'pain' more likely to suggest a phenomenal reading than perceptual terms such as 'see'.

Although it is not clear why he compares "see" to "pain" rather than "feel", it is clear enough from this passage that he treats "pain" as a term referring to a sensation. However, as already noted in $\S 4.1$, laypeople tend to locate pains in the body rather than the mind. Further, while the passage attempts to explain away SM's results based on the ambiguity between functional and phenomenal readings of mental terms, this is just a version of the Ambiguity Objection and we've seen in $§ 4.3$ and Chapter 5 that this objection does not hold water.

To sum up, the scarcity of existence intuitions suggests that distribution and quality intuitions are not prevalent. More importantly, pace Chalmers, it also suggests that it is unrealistic to expect that 
"central" problem intuitions are widespread. That said, this implication of the scarcity of existence intuitions for the meta-problem of consciousness is nonetheless indirect since the meta-problem concerns mainly the central problem intuitions. However, Chapters 7-8 provide us with empirical data that are directly relevant to assessing the prevalence of the central problem intuitionsexplanatory, modal and metaphysical intuitions - as detailed in the next sub-section.

\subsubsection{Prevalence of Central Problem Intuitions}

As noted, Chalmers lists explanatory, metaphysical, knowledge and modal intuitions as four kinds of central intuitions. As will be clearer, three of these (explanatory, knowledge, and modal intuitions) roughly correspond with what I called epistemic gap intuitions in Chapter 3 under the names of explanatory gap, knowledge, and conceivability intuitions. Chapters 7-8 investigated the prevalence of explanatory and conceivability/modal intuitions and concluded that they are not shared by most laypeople. In the rest of this sub-section, I will summarize the empirical evidence that backs up this conclusion and how this evidence straightforwardly shows that central problem intuitions are not prevalent.

Chalmers divides explanatory intuitions into two main kinds: explanatory gap intuitions (phenomenal consciousness poses a hard problem) and anti-functionalist intuitions that reflect that "explaining behavioural functions does not suffice to explain consciousness" (Chalmers, 2018, p. 12). The studies discussed in $\$ 7.2$ and Chapter 8 - Gottlieb and Lombrozo (2018), Díaz (2021) and Explanatory Studies 1 and 2-indicate that laypeople by and large do not share explanatory gap intuitions. To briefly repeat, Gottlieb and Lombrozo (2018) found that laypeople, by and large, do not take issue with the possibility of scientific explanation of various traits, abilities or phenomena that philosophers would associate with phenomenal consciousness. Díaz (2021) and Explanatory Study 2 indicates that the majority of people do not share the intuition that so-called phenomenal states such as feeling pain and seeing colors cannot be fully explained by neuroscience and the like. Explanatory Study 1 also indicates that explanatory gap intuitions are not prevalent in that only a minority of participants held that, unlike robots, humans cannot be fully explained 
by natural sciences because there is something about us that is beyond the reach of scientific explanation.

As for modal intuitions, or conceivability intuitions as I called them, Chalmers states that these intuitions concern "what is possible or conceivable" and subsume what I called philosophical zombie intuition (the intuition that "physical or functional duplicate of us might lack consciousness") and inversion intuitions reflecting for example that "someone else might be experiencing red when I experience green" (p.12). As discussed in §7.1.2 and §7.1.3, Peressini (2014) and Fischer and Sytsma (2021) suggest that laypeople for the most part lack philosophical zombie intuitions. That is, they do not seem to have the intuition that there might be some creatures that are physically and behaviourally indistinguishable from us but lack an inner, "phenomenal" life. Also, as noted in §7.1.1, Sytsma (2010) found that participants by and large denied that an inverted spectrum case is possible, suggesting not only that experiential property intuitions but also what Chalmers (2018, p. 12) calls "inversion intuitions" are not prevalent. These two sets of studies collectively suggest that laypeople by and large do not share modal intuitions.

Lastly, Chalmers treats "dualist intuitions" that reflect that phenomenal consciousness is nonphysical and "fundamentality intuitions" that reflect that phenomenal consciousness is fundamental or simple as the two main kinds of metaphysical intuitions (p.12). The empirical evidence suggests that dualist intuitions are not prevalent either. As we saw, Díaz (2021) suggests that the majority of people tend to think that so-called phenomenal states are identical with particular brain states. Also remember that in Explanatory Study 1, only a minority of participants held that humans, unlike robots, are not identical to their bodies.

Based on the empirical evidence listed above, it seems clear that even if the implications of the scarcity of existence intuitions are somehow dismissed as indirect, the studies discussed in Chapters 7-8 provide solid evidence that three major kinds of central problem intuitions (explanatory, metaphysical, and modal intuitions) are not prevalent. As such, Chalmers's empirical hypothesis seems to be incorrect. From this, does it follow that there is no meta-problem? In the next sub-section, I will discuss this question. 


\subsubsection{Does the Meta-problem Survive the Scarcity of Problem Intuitions?}

Given the scarcity of problem intuitions, if the meta-problem is understood in its presumptuous sense, we can straightforwardly conclude that there is no meta-problem simply because "we" by and large do not share problem intuitions. However, as noted, in addition to the initial presumptuous definition of the problem, Chalmers also operates with a modest construal of the meta-problem under which it is simply the problem of explaining problem intuitions where they are present and the variability in these intuitions.

The problem in its modest sense survives the scarcity of problem intuitions. However, it is not difficult to see that the problem is decidedly less important under such a definition. This is because, as Chalmers (2020, pp. 243-244) admits, if problem intuitions are not widespread, then it is more likely that they are wrong and that the source of problem intuitions is not phenomenal consciousness but special factors. That is, if problem intuitions are not widespread, then we have good reason to think that they do not have a widely shared, universal basis related to phenomenal consciousness, and thus that Chalmers's working hypothesis is mistaken.

Chalmers (2018, p. 15) claims that even if his working hypothesis is false, the meta-problem still remains important as the problem of explaining problem intuitions in terms of variable factors. However, if problem intuitions do not have a universal basis and are due to variable (cultural, historical reasons, theoretical background and so on) factors, uncovering such factors would not have nearly the same importance as finding a universal basis, at least for consciousness studies.

Further, if Chalmers's working hypothesis is mistaken, then the meta-problem loses much of its attraction for philosophers and scientists working on phenomenal consciousness, simply because problem intuitions would be less likely to shed light on the problem of phenomenal consciousness, as Chalmers (2020, p. 243) admits. The reverse is also true. As will be detailed in $\S 10.3$, explaining phenomenal consciousness would not provide any explanation of why some people have problem intuitions. 
Overall, the evidence suggests, first, that there is no meta-problem in its presumptuous sense because "we" by and large lack problem intuitions. And in the modest sense, the meta-problem becomes less important and attractive for philosophers and scientists working on consciousness. As such, in either sense, the results discussed in this thesis deflate the so-called meta-problem of consciousness.

\subsection{The Reverse Meta-Problem}

In brief, the meta-problem of consciousness is the problem of explaining our problem intuitions. The evidence indicates that such problem intuitions are not widespread, however. Given the seeming paucity of problem intuitions, I hold that the framing for the meta-problem points us in the wrong direction: whether articulated in its presumptive or modest form, it suggests that what calls for explanation is our problem intuitions. But we've now seen that the occurrence of problem intuitions is the outlier and not the default. As such, I contend that what most calls out for explanation is our general lack of problem intuitions. This is the reverse meta-problem. And I suggest that there is a simple, straightforward answer to this problem: most people lack problem intuitions because phenomenal consciousness doesn't exist. I will not specifically defend this solution, here, but rather take it to raise a serious challenge for phenomenal realists: The challenge is to articulate why we should assume that phenomenal consciousness exists given the paucity of intuitive evidence for it.

Chalmers (2018) holds that the meta-problem and the hard problem of consciousness are closely connected, and he contends that we should expect that a solution to one of these problems will cast light on the other. This leads to what Chalmers calls the "meta-problem challenge": "If a theory says that mechanism $\mathrm{M}$ is the basis of consciousness, then it needs to explain how mechanism $\mathrm{M}$ plays a central role in bringing about judgments about consciousness" (p. 36). Like the metaproblem itself, this challenge focuses us in a certain direction. In essence, it treats explaining our problem intuitions as a desideratum for theories of phenomenal consciousness. But we've now seen that problem intuitions are scarce. As such, the better desideratum would seem to be to explain the general absence of problem intuitions on the assumption of a given account of phenomenal 
consciousness. And I believe this is rather pointed: realist accounts owe us compelling reasons to take the supposed phenomenon of phenomenal consciousness seriously given the dearth of intuitive support for it.

I noted above that the scarcity of problem intuitions indicates against Chalmers's working hypothesis that there are near-universal factors related to phenomenal consciousness that play a central, underlying role in producing problem intuitions and reports. If this is correct, then we should not expect any explanation of phenomenal consciousness to explain the problem intuitions of the minority. This is because the source of problem intuitions would now seem to be variable factors rather than universal factors related to phenomenal consciousness, the opposite of what Chalmers hypothesizes. The basic idea is that if the source of problem intuitions is not phenomenal consciousness-if phenomenal consciousness does not play a role in producing problem intuitions - then we should not expect a theory of phenomenal consciousness to explain problem intuitions. In other words, if Chalmers's working hypothesis is incorrect, as it seems to be, then the meta-problem challenge becomes inapposite.

Chalmers (2020, p. 243) admits this; however, he nonetheless claims that a central version of the meta-problem challenge would still survive the scarcity of problem intuitions. This version applies not only to problem reports such as "feeling pain cannot be fully explained in physical terms" but also to simple phenomenal reports that he thinks are contained in saying, e.g., "I am conscious". So, he claims that "what explains consciousness should also help explain these judgments and reports" (p. 244). That is, he seems to think that a theory of phenomenal consciousness should explain at least simple phenomenal reports if not problem reports.

However, a theory of phenomenal consciousness could only be in a position to explain these simple reports if they really report on or refer to phenomenal consciousness. Only in this case would we have reason to think that the source of these reports is connected to phenomenal consciousness, and thus to think that a theory of phenomenal consciousness could help explain these reports. But why should we assume that such simple reports are reports of phenomenal consciousness? Chalmers is silent on this. He just assumes that they should be interpreted as referring to 
phenomenally conscious states without giving an independent reason for why they should be interpreted in this way. But the evidence we've seen suggests not only that the source of problem intuitions is not phenomenal consciousness itself, but also that laypeople do not generally share existence intuitions. And if existence intuitions are not prevalent, then there is no reason to assume that people mean something like phenomenal consciousness when they talk about consciousness, or that they imply something about phenomenal consciousness when they talk about feeling pains, seeing colors, or other so-called phenomenal experiences.

In fact, the scarcity of existence and epistemic gap intuitions itself raises difficulties for phenomenal realism. We've seen that the standard defence of the existence of phenomenal consciousness appeals to intuitions. But if phenomenal consciousness existed and was such that our intuitions serve as compelling evidence for it, then we would expect laypeople to recognize the common phenomenality of so-called phenomenal states and, thus, to generally share philosophers' phenomenal intuitions. But the evidence strongly suggests that they do not. Unless only philosophers are phenomenally conscious entities, this shows that the main, intuition-driven way of defending phenomenal realism fails. And considering that it is difficult, at best, to find nonintuition driven "evidence" for the existence of phenomenal consciousness, we do not seem to have any plausible reason at present to assume that phenomenal realism is true. As such, we have reason to tentatively conclude that phenomenal consciousness is simply a philosophical fiction.

Based on these considerations, I charge that any realist theory of phenomenal consciousness should explain why we should assume that phenomenal consciousness exists in the first place and do so in a way that is consistent with the general paucity of phenomenal intuitions. In other words, phenomenal realists face the challenge of explaining why we should believe that phenomenal consciousness exists given that the main way of defending phenomenal realism fails, as the General Argument concludes. Realist theories of phenomenal consciousness should respond to this challenge rather than taking up the unwarranted meta-problem challenge. That is, phenomenal realists should attempt to provide non-intuitional evidence indicating that phenomenal consciousness is real rather than trying to explain why "we" have intuitions reflecting that 
phenomenal consciousness poses a hard problem. The reason is simple: the evidence indicates that we do not have such intuitions. 


\section{REFERENCES}

Alter, T. (2006). Does representationalism undermine the Knowledge Argument? In T. Alter \& S. Walter (Eds.), Phenomenal concepts and phenomenal knowledge: New essays on consciousness and physicalism (pp. 65-76): Oxford University Press.

Arico, A. (2010). Folk psychology, consciousness, and context effects. Review of Philosophy and Psychology, 1(3), 371-393.

Baars, B. J. (1993). A cognitive theory of consciousness: Cambridge University Press.

Bailey, A. (2007). Qualia and the argument from illusion: A defence of figment. Acta Analytica, 22(2), 85-103.

Balog, K. (2009). Phenomenal concepts. In B. McLaughlin, A. Beckermann, \& S. Walter (Eds.), The Oxford handbook of philosophy of mind: Oxford University Press.

Balog, K. (2012). Acquaintance and the mind-body problem. In S. Gozzano \& C. S. Hill (Eds.), New perspectives on type identity: The mental and the physical (pp. 16-42).

Block, N. (1978). Troubles with functionalism. Minnesota Studies in the Philosophy of Science, 9 , 261-325.

Block, N. (1990). Inverted earth. Philosophical Perspectives, 4, 53-79.

Block, N. (2002). Some concepts of consciousness. In D. J. Chalmers (Ed.), Philosophy of mind: Classical and contemporary readings (pp. 206-229). Oxford: Oxford University Press.

Block, N. (2004). Qualia. In R. Gregory (Ed.), Oxford companion to the mind: Oxford University Press.

Bogardus, T. (2009). A vindication of the equal-weight view. Episteme, 6(3), 324-335.

Bourget, D., \& Chalmers, D. J. (2014). What do philosophers believe? Philosophical Studies, $170(3), 465-500$.

Byrne, A. (2004). What phenomenal consciousness is like. Advances in Consciousness Research, 56, 203-226.

Byrne, A. (2020). Inverted qualia. In E. N. Zalta (Ed.), The Stanford encyclopaedia of philosophy. Retrieved from https://plato.stanford.edu/archives/fall2020/entries/qualia-inverted 
Campbell, J. (2002). Reference and consciousness: Oxford University Press.

Carruthers, P. (2001). Consciousness: Explaining the phenomena. Royal Institute of Philosophy Supplements, 49, 61-85.

Carruthers, P., \& Veillet, B. (2007). The phenomenal concept strategy. Journal of Consciousness Studies, 14(9-10), 212-236.

Chalmers, D. J. (1995). Facing up to the problem of consciousness. Journal of Consciousness Studies, 2(3), 200-219.

Chalmers, D. J. (1996). The conscious mind: In search of a fundamental theory: Oxford University Press.

Chalmers, D. J. (2002). Consciousness. In D. J. Chalmers (Ed.), Philosophy of mind: Classical and contemporary readings: Oxford University Press.

Chalmers, D. J. (2003). Consciousness and its place in nature. In S. P. Stich \& T. A. Warfield (Eds.), Blackwell guide to the philosophy of mind (pp. 102-142): Blackwell.

Chalmers, D. J. (2009). The two-dimensional argument against materialism. In A. Beckermann, B. P. McLaughlin, \& S. Walter (Eds.), Oxford handbook of philosophy of mind: Oxford University Press.

Chalmers, D. J. (2010). The character of consciousness: Oxford University Press.

Chalmers, D. J. (2018). The meta-problem of consciousness. Journal of Consciousness Studies, 25(9-10), 6-61.

Chalmers, D. J. (2020). Is the hard problem of consciousness universal? Journal of Consciousness Studies, 27(5-6), 227-257.

Christensen, D. (2007). Epistemology of disagreement: The good news. The Philosophical Review, 116(2), 187-217.

Churchland, P. M. (1989). Knowing qualia: A reply to Jackson. In P. Ludlow, Y. Nagasawa, \& D. Stojar (Eds.), There's Something About Mary: Essays on phenomenal consciousness and Frank Jackson's knowledge argument. Cambridge: The MIT Press.

Cottingham, J., Stoothoff, R., Murdoch, D., \& Kenny, A. (1985). The philosophical writings of Descartes (Vol. 1): Cambridge University Press.

Crick, F., \& Clark, J. (1994). The astonishing hypothesis. Journal of Consciousness Studies, 1(1), $10-16$. 
Dehaene, S., \& Naccache, L. (2001). Towards a cognitive neuroscience of consciousness: Basic evidence and a workspace framework. Cognition, 79(1-2), 1-37.

Dennett, D. C. (1988). Quining qualia. In D. J. Chalmers (Ed.), Philosophy of mind: Classical and contemporary readings: Oxford University Press.

Dennett, D. C. (1991). Consciousness explained: Little, Brown and Company.

Dennett, D. C. (2001). The zombic hunch: Extinction of an intuition? Royal Institute of Philosophy Supplements, 48, 27-43.

Díaz, R. (2021). Do people think consciousness poses a hard problem?: Empirical evidence on the meta-problem of consciousness. Journal of Consciousness Studies, 28(3-4), 55-75.

Dretske, F. I. (2003). Experience as representation. Philosophical issues, 13, 67-82.

Elga, A. (2007). Reflection and disagreement. Noûs, 41(3), 478-502.

Feldman, R. (2006). Epistemological puzzles about disagreement. Epistemology futures, 216-236.

Fiala, B., Arico, A., \& Nichols, S. (2014). I, robot. In E. Machery \& E. O'Neill (Eds.), Current controversies in experimental philosophy: Routledge.

Fischer, E., \& Engelhardt, P. E. (2017). Stereotypical inferences: Philosophical relevance and psycholinguistic toolkit. Ratio, 30(4), 411-442.

Fischer, E., \& Sytsma, J. (2021). Zombie intuitions. Cognition, 215. doi:104807

Frances, B., \& Matheson, J. (2019). Disagreement. In E. N. Zalta (Ed.), The Stanford encyclopedia of philosophy.

Frankish, K. (2012). Quining diet qualia. Consciousness and Cognition, 21(2), 667-676.

Frankish, K. (2016a). Illusionism as a theory of consciousness. Journal of Consciousness Studies, 23(11-12).

Frankish, K. (2016b). Not disillusioned: Reply to commentators. Journal of Consciousness Studies, 23(11-12), 256-289.

Frederick, S. (2005). Cognitive reflection and decision making. Journal of Economic Perspectives, 19(4), 25-42.

Goff, P. (2012). A priori physicalism, lonely ghosts and cartesian doubt. Consciousness and Cognition, 21(2), 742-746. 
Gottlieb, S., \& Lombrozo, T. (2018). Can science explain the human mind? Intuitive judgments about the limits of science. Psychological Science, 29(1), 121-130.

Gray, H. M., Gray, K., \& Wegner, D. M. (2007). Dimensions of mind perception. Science, 315(5812), 619-619.

Hales, S. D. (2009). Relativism and the foundations of philosophy: The MIT Press.

Harman, G. (1990). The intrinsic quality of experience. Philosophical Perspectives, 4, 31-52.

Horgan, T. (1984). Jackson on physical information and qualia. The Philosophical Quarterly, 34(135), 147-152.

Huebner, B. (2010). Commonsense concepts of phenomenal consciousness: Does anyone care about functional zombies? Phenomenology and the Cognitive Sciences, 9(1), 133-155.

Huemer, M. (2011). Phenomenal conservatism and self-defeat: A reply to DePoe. Philosophical Studies, 156(1), 1-13.

Jackendoff, R. (1987). Consciousness and the computational mind: The MIT Press.

Jackson, F. (1982). Epiphenomenal qualia. In P. Ludlow, Y. Nagasawa, \& S. Daniel (Eds.), There's Something About Mary: Essays on phenomenal consciousness and Frank Jackson's knowledge argument. Cambridge: The MIT Press.

Jackson, F. (1986). What Mary didn't know. In P. Ludlow, Y. Nagasawa, \& D. Stojar (Eds.), There's Something About Mary: Essays on phenomenal consciousness and Frank Jackson's knowledge argument. Cambridge: The MIT Press.

Jaworski, W. (2011). Philosophy of mind: A comprehensive introduction: John Wiley \& Sons.

Kelly, T. (2013). Disagreement and the burdens of judgment. The epistemology of disagreement: New essays, 31-53.

Kim, H.-e., Poth, N., Reuter, K., \& Sytsma, J. (2016). Where is your pain? A cross-cultural comparison of the concept of pain in Americans and South Koreans. Studia Philosophica Estonia, 9(1), 136-169.

Kind, A. (2003). What's so transparent about transparency? Philosophical Studies, 115(3), 225244.

Kind, A. (2008). How to believe in qualia. In E. Wright (Ed.), The case for qualia (pp. 285). 
Kind, A. (2020). Qualia. In J. Fieser \& B. Dowden (Eds.), Internet encyclopedia of philosophy. Retrieved from https://www.iep.utm.edu/qualia/

Knobe, J., \& Prinz, J. (2008). Intuitions about consciousness: Experimental studies. Phenomenology and the Cognitive Sciences, 7(1), 67-83. doi:10.1007/s11097-007-9066-y

Koch, C. (2004). The quest for consciousness: A neurobiological approach. Denver, Colo: Roberts and Co.

Kozak, M. N., Marsh, A. A., \& Wegner, D. M. (2006). What do i think you're doing? Action identification and mind attribution. Journal of Personality and Social Psychology, 90(4), 543.

Kripke, S. A. (1980). Naming and necessity: Basil Blackwell.

Lackey, J. (2010). What should we do when we disagree? In T. S. Gendler \& J. Hawthorne (Eds.), Oxford studies in epistemology (Vol. 3).

Leeds, S. (1993). Qualia, awareness, Sellars. Nô̂s, 27(3), 303-330.

Levine, J. (1983). Materialism and qualia: The explanatory gap. Pacific philosophical quarterly, 64(4), 354-361.

Levine, J. (2001). Purple haze: The puzzle of consciousness: Oxford University Press.

Lewis, D. (1988). What experience teaches. In P. Ludlow, Y. Nagasawa, \& D. Stojar (Eds.), There's something about Mary: Essays on phenomenal consciousness and Frank Jackson's knowledge argument. Cambridge: The MIT Press.

Loar, B. (1990). Phenomenal states. In P. Ludlow, Y. Nagasawa, \& D. Stojar (Eds.), There's something about mary: Essays on phenomenal consciousness and frank jackson's knowledge argument. Cambridge: The MIT Press.

Locke, J. (1689/1975). An essay concerning human understanding. Oxford: Oxford University Press.

Ludwig, K. (2007). The epistemology of thought experiments: First person versus third person approaches. Midwest studies in philosophy, 31, 128-159.

Machery, E., Mallon, R., Nichols, S., \& Stich, S. P. (2004). Semantics, cross-cultural style. Cognition, 92(3), B1-B12. 
Malle, B. F. (2019). How many dimensions of mind perception really are there. Paper presented at the Proceedings of the 41st Annual Meeting of the Cognitive Science Society. Austin, TX: Cognitive Science Society.

Marshall, D., \& Weatherson, B. (2018). Intrinsic vs. Extrinsic properties. In E. N. Zalta (Ed.), The Stanford encyclopedia of philosophy.

Musacchio, J. M. (2002). Dissolving the explanatory gap: Neurobiological differences between phenomenal and propositional knowledge. Brain and Mind(3), 331-365.

Nagasawa, Y., \& Stoljar, D. (2004). Introduction. In P. Ludlow, Y. Nagasawa, \& D. Stoljar (Eds.), There's something about Mary: Essays on phenomenal consciousness and Frank Jackson's knowledge argument. Cambridge: The MIT Press.

Nagel, T. (1974). What is it like to be a bat? The Philosophical Review, 83(4), 435-450.

Nemirow, L. (2006). So this is what it's like a defense of the ability hypothesis. In T. Alter \& S. Walter (Eds.), Phenomenal concepts and phenomenal knowledge: New essays on consciousness and physicalism: Oxford University Press.

Nida-Rümelin, M. (2021). Qualia: The knowledge argument. In E. N. Zalta (Ed.), The Stanford encyclopedia of philosophy. Retrieved from https://plato.stanford.edu/archives/sum2021/entries/qualia-knowledge.

Papineau, D. (2006). Phenomenal and perceptual concepts. In T. Alter \& S. Walter (Eds.), Phenomenal concepts and phenomenal knowledge. New York: Oxford University Press.

Papineau, D. (2007). Kripke's proof is ad hominem not two-dimensional. Philosophical Perspectives, 21, 475-494.

Peressini, A. (2014). Blurring two conceptions of subjective experience: Folk versus philosophical phenomenality. Philosophical Psychology, 27(6), 862-889.

Perry, J. (2001). The 1999 Jean Nicod lectures: Knowledge, possibility and consciousness: The MIT Press.

Phelan, M., Arico, A., \& Nichols, S. (2013). Thinking things and feeling things: On an alleged discontinuity in folk metaphysics of mind. Phenomenology and the Cognitive Sciences(12), 703-725.

Pituch, K. A., \& Stevens, J. P. (2015). Applied multivariate statistics for the social sciences: Analyses with SAS and IBM's SPSS (6 ed.): Routledge.

Prinz, J. (2012). The conscious brain: Oxford University Press. 
Pust, J. (2019). Intuition. In E. N. Zalta (Ed.), The Stanford encyclopedia of philosophy. Retrieved from https://plato.stanford.edu/archives/sum2019/entries/intuition/

Reuter, K., Phillips, D., \& Sytsma, J. (2014). Hallucinating pain. In J. Sytsma (Ed.), Advances in experimental philosophy of mind: Bloomsbury.

Reuter, K., \& Sytsma, J. (2018). Unfelt pain. Synthese, 197(4), 1777-1801.

Robinson, W. S. (2004). Understanding phenomenal consciousness: Cambridge University Press.

Schwitzgebel, E. (2016). Phenomenal consciousness, defined and defended as innocently as i can manage. Journal of Consciousness Studies, 23(11-12), 224-235.

Schwitzgebel, E., \& Cushman, F. (2012). Expertise in moral reasoning? Order effects on moral judgment in professional philosophers and non-philosophers. Mind \& Language, 27(2), 135-153.

Sorensen, R. (2014). Novice thought experiments. In A. R. Booth \& D. P. Rowbottom (Eds.), Intuitions: Oxford University Press.

Stoljar, D. (2006). Ignorance and imagination: The epistemic origin of the problem of consciousness: Oxford University Press.

Sytsma, J. (2009). Phenomenological obviousness and the new science of consciousness. Philosophy of Science, 76(5), 958-969.

Sytsma, J. (2010). Dennett's theory of the folk theory of consciousness. Journal of Consciousness Studies, 17(3-4), 107-130.

Sytsma, J. (2012). Revisiting the valence account. Philosophical Topics, 179-198.

Sytsma, J. (2014). The robots of the dawn of experimental philosophy of mind. In E. Machery \& E. O'Neill (Eds.), Current controversies in experimental philosophy: Routledge.

Sytsma, J. (2018). Experiencers and the ambiguity objection. Retrieved from http://philsciarchive.pitt.edu/15481/

Sytsma, J., \& Livengood, J. (2015). The theory and practice of experimental philosophy: Broadview Press.

Sytsma, J., \& Machery, E. (2009). How to study folk intuitions about phenomenal consciousness. Philosophical Psychology, 22(1), 21-35. 
Sytsma, J., \& Machery, E. (2010). Two conceptions of subjective experience. Philosophical Studies(151), 299-327.

Sytsma, J., \& Machery, E. (2012). On the relevance of folk intuitions: A commentary on Talbot. Consciousness and Cognition, 21(2), 654-660.

Sytsma, J., \& Ozdemir, E. (2019). No problem: Evidence that problem intuitions are not widespread. Journal of Consciousness Studies, 26(9-10), 241-256.

Sytsma, J., \& Reuter, K. (2017). Experimental philosophy of pain. Journal of Indian Council of Philosophical Research, 34(3), 611-628.

Talbot, B. (2012). The irrelevance of folk intuitions to the "hard problem" of consciousness. Consciousness and Cognition, 21(2), 644-650.

Thomson, K. S., \& Oppenheimer, D. M. (2016). Investigating an alternate form of the cognitive reflection test. Judgment and Decision making, 11(1), 99.

Tobia, K., Buckwalter, W., \& Stich, S. (2013). Moral intuitions: Are philosophers experts? Philosophical Psychology, 26(5), 629-638.

Tye, M. (2000). Consciousness, color, and content (representation and mind): MIT Press.

Tye, M. (2018). Qualia. In E. N. Zalta (Ed.), The Stanford encyclopedia of philosophy. Retrieved from https://plato.stanford.edu/entries/qualia/

Van Inwagen, P. (1996). Is it wrong, everywhere, always, and for anyone to believe anything on insufficient evidence? In J. Jordan \& D. Howard-Snyder (Eds.), Faith, freedom, and rationality: Philosophy of religion today (pp. 136-153): Rowman \& Littlefield.

Weisman, K., Dweck, C. S., \& Markman, E. M. (2017). Rethinking people's conceptions of mental life. Proceedings of the National Academy of Sciences, 114(43), 11374-11379. 